\title{
A Synthesis of Human-related Avian Mortality in Canada
}

\section{Synthèse des sources de mortalité aviaire d'origine anthropique au Canada}

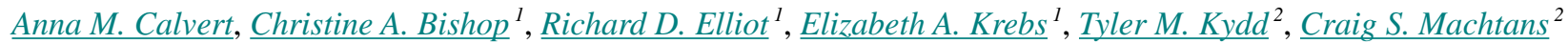 \\ and Gregory J. Robertson ${ }^{1}$
}

\begin{abstract}
Many human activities in Canada kill wild birds, yet the relative magnitude of mortality from different sources and the consequent effects on bird populations have not been systematically evaluated. We synthesize recent estimates of avian mortality in Canada from a range of industrial and other human activities, to provide context for the estimates from individual sources presented in this special feature. We assessed the geographic, seasonal, and taxonomic variation in the magnitude of national-scale mortality and in population-level effects on species or groups across Canada, by combining these estimates into a stochastic model of stage-specific mortality. The range of estimates of avian mortality from each source covers several orders of magnitude, and, numerically, landbirds were the most affected group. In total, we estimate that approximately 269 million birds and 2 million nests are destroyed annually in Canada, the equivalent of over 186 million breeding individuals. Combined, cat predation and collisions with windows, vehicles, and transmission lines caused $>95 \%$ of all mortality; the highest industrial causes of mortality were the electrical power and agriculture sectors. Other mortality sources such as fisheries bycatch can have important local or species-specific impacts, but are relatively small at a national scale. Mortality rates differed across species and families within major bird groups, highlighting that mortality is not simply proportional to abundance. We also found that mortality is not evenly spread across the country; the largest mortality sources are coincident with human population distribution, while industrial sources are concentrated in southern Ontario, Alberta, and southwestern British Columbia. Many species are therefore likely to be vulnerable to cumulative effects of multiple human-related impacts. This assessment also confirms the high uncertainty in estimating human-related avian mortality in terms of species involved, potential for population-level effects, and the cumulative effects of mortality across the landscape. Effort is still required to improve these estimates, and to guide conservation efforts to minimize direct mortality caused by human activities on Canada's wild bird populations. As avian mortality represents only a portion of the overall impact to avifauna, indirect effects such as habitat fragmentation and alteration, site avoidance, disturbance, and related issues must also be carefully considered.
\end{abstract}

RÉSUMÉ. Au Canada, de nombreuses activités d'origine anthropique entraînent la mort d'oiseaux sauvages, mais l'ampleur relative de la mortalité selon les diverses sources et leurs conséquences sur les populations d'oiseaux n'ont pas été évaluées systématiquement. Nous avons compilé des estimations récentes de mortalité aviaire au Canada causée par des activités industrielles et d'autres origines anthropiques afin de mettre en contexte les estimations calculées pour chacune des sources de mortalité présentées dans ce numéro spécial. Nous avons évalué la variation géographique, saisonnière et taxinomique de l'ampleur de la mortalité à l'échelle nationale, de même que les effets sur les populations d'espèces ou de groupes dans l'ensemble du Canada. Nous avons ensuite combiné ces estimations dans un modèle stochastique de mortalité spécifique au stade de vie. L'étendue des estimations de la mortalité par les diverses sources couvre plusieurs ordres de grandeur et les oiseaux terrestres sont le groupe le plus affecté en termes de nombre. Dans l'ensemble, nous avons estimé qu'approximativement 276 millions d'oiseaux et 2 millions de nids sont détruits chaque année au Canada, soit l'équivalent de plus de 188 millions d'individus nicheurs. La prédation par les chats et les collisions mortelles avec les fenêtres, les véhicules et les lignes de transmission ont été collectivement responsables de > $95 \%$ de la mortalité; les sources industrielles de mortalité les plus importantes ont été les secteurs de la production d'énergie et de l'agriculture. Par ailleurs, les sources de mortalité comme les prises accidentelles par les pêcheries peuvent avoir d'importants impacts locaux ou propres à une espèce, mais ces impacts sont relativement faibles à l'échelle nationale. Les taux de mortalité variaient selon les espèces et les familles au sein des principaux groupes d'oiseaux, soulignant le fait que la mortalité n'est pas simplement proportionnelle à l'abondance. Nous avons aussi constaté que la mortalité n'est pas uniforme dans l'ensemble du pays : les sources de mortalité les plus importantes coïncident avec les foyers de population

${ }^{1}$ Environment Canada, Wildlife Research Division, Wildlife and Landscape Science Directorate, ${ }^{2}$ Environment Canada, Canadian Wildlife Service

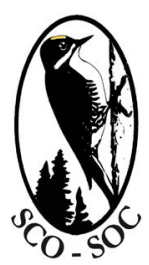

Sponsored by the Society of Canadian Ornithologists and Bird Studies Canada

Parrainée par la Société des ornithologistes du Canada et Études d'oiseaux Canada

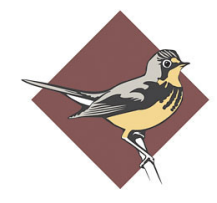

BIRD STUDIES CANADA 
humaine, alors que les sources industrielles sont concentrées dans le sud de l'Ontario, en Alberta et dans le sud-ouest de la Colombie-Britannique. De nombreuses espèces sont donc vraisemblablement vulnérables aux effets cumulatifs des multiples impacts de sources anthropiques. Notre évaluation confirme aussi les grandes incertitudes liées à l'estimation de la mortalité aviaire d'origine anthropique en matière d'espèces touchées, d'effets potentiels sur le plan des populations et d'effets cumulatifs de la mortalité à l'échelle du paysage. Les efforts doivent être poursuivis afin d'améliorer ces estimations et d'orienter les actions de conservation pour minimiser la mortalité directe causée par les activités d'origine anthropique sur les populations aviaires du Canada. Puisque la mortalité aviaire ne représente qu'une partie de l'ensemble des impacts sur l'avifaune, les effets indirects tels que la fragmentation et la perturbation d'habitats, l'évitement de sites précis, le dérangement et autres enjeux connexes doivent également être considérés attentivement.

Key Words: bird mortality; cats; collisions; human impacts; incidental take; industry; population effects

\section{INTRODUCTION}

Several billion birds from over 400 species breed each year in Canada (Blancher 2002), in a wide variety of habitats. Landbirds, i.e., songbirds, raptors, upland gamebirds, represent most of the birds in Canada and tend to have large and widespread populations. Aquatic birds, such as waterfowl, seabirds, shorebirds, and inland waterbirds, occupy freshwater and marine habitats across the country. Birds occupy diverse niches across Canada that overlap substantially with human activities, and so are vulnerable to a large range of humanrelated stressors. The recent State of Canada's Birds report (NABCI-Canada 2012) highlighted conservation efforts that have contributed to increases in waterfowl and raptor populations, but shorebirds, grassland birds, and aerial insectivores have experienced rapid declines, some of which are attributed to human-driven habitat change and mortality across North America over the past 40 years (NABCI-Canada 2012).

Direct mortality resulting from human activities may have important consequences, particularly when it is additive to natural mortality, i.e. if individuals killed would have otherwise survived (Anderson and Burnham 1976). Agricultural practices, for example, have been identified as a factor in declines of Northern Pintail (Anas acuta; Miller and Duncan 1999, Prairie Habitat Joint Venture 2008) and Bobolink (Dolichonyx oryzivorus; COSEWIC 2010) as well as U.S. grassland birds (Mineau and Whiteside 2013), while reduced juvenile survivorship and population declines of urban songbirds have been linked to predation by cats (Crooks and Soulé 1999, Balogh et al. 2011). Quantification of the magnitude of human-related avian mortality, and its population-level effects on Canada's birds, is essential for directing management and conservation actions and for prioritizing future research directions (Loss et al. 2012); especially when considered in conjunction with indirect stressors such as habitat alteration and climate change.

Preventing and minimizing human-related mortality to birds, their nests, and eggs is widely supported by environmental legislation in Canada. Federal and provincial governments are responsible for the protection, conservation, and management of birds under the federal Migratory Birds Convention Act (S.
C. 1994, c. 22), the federal Species at Risk Act (S.C. 2002, c. 29) and various provincial wildlife Acts. These laws generally prohibit the destruction of nests and eggs, and the "take" or killing of individual birds. Permitting systems exist to manage direct mortality due to hunting or while preventing damage and danger to the public, but provisions or systems to authorize inadvertent destruction of nests or birds as a consequence of anthropogenic activities, often called 'incidental take,' are applicable only to limited species or circumstances. Activities that may destroy nests or birds are currently managed through compliance promotion and by providing relevant information, e.g., timing of breeding seasons, key migration periods and pathways, to industrial sectors. This information allows the development and adoption of measures that minimize the risk of inadvertent destruction of nests and eggs, or killing of individuals.

Some sources of human-related avian mortality are wellquantified, such as the regulated sport harvest of game birds, but the magnitudes of most sources are imprecise or unknown. In particular, those affecting a few birds at a time, e.g., cat predation or building collisions, may often be overlooked because their local effects are rarely extrapolated nationally. Therefore, the number of birds killed annually in Canada as a result of human activities is poorly known, as are any resulting effects on populations. Despite limitations imposed by smallscale studies, nonrandom sampling designs, and an absence of experimental controls (Loss et al. 2012), preliminary estimates of human-related bird mortality at national- or continental-level scales can be highly informative. For instance, mortality from collisions with communication towers results in a total annual kill across the U.S. and Canada of about 6.8 million birds (Longcore et al. 2012), include disproportionately large impacts on certain species, many of conservation concern (Longcore et al. 2013). These studies can further highlight the susceptibility of particular bird groups to certain mortality sources, such as the vulnerability of longdistance or nocturnal migrants to collisions with towers and buildings (Klem 2009, Manville 2009, Arnold and Zink 2011) or of auks to bycatch in gill nets (Piatt et al. 1984).

The papers presented in this special feature of Avian Conservation and Ecology reflect the current scientific 
understanding of the magnitude of human-related bird mortality in Canada, based on data collected from a variety of industrial and other activities. Each paper reports an estimate of the total annual loss of birds, nests, or eggs, and considers the likelihood of population-level effects on species in Canada. In this synthesis, we compare the relative contribution of each source of mortality, including several estimates that are unpublished or were published recently elsewhere, and consider the implications of the total kill from all sources. Specifically, this synthesis aims to (i) identify, quantify, and compare sources of human-related avian mortality in Canada, (ii) explicitly model the sources of uncertainty in the mortality estimates, (iii) identify the remaining gaps in the current knowledge of threats to Canadian bird populations, and (iv) thereby help to prioritize research, policy, management, and conservation actions aimed at understanding and reducing human-related bird mortality in Canada.

\section{METHODS}

\section{Sources of mortality}

We synthesized estimates of the magnitude of human-related avian mortality in Canada from major industrial sectors and nonindustrial or public activities that we believe kill substantial numbers of birds. Initial estimates were developed in a series of reports prepared for Environment Canada. Nine of these are found in this special feature, namely mortality caused by: collisions with vehicles (Bishop and Brogan 2013), cats (Blancher 2013), marine industries, i.e., offshore oil and gas, commercial fisheries (Ellis et al. 2013), commercial forestry (Hobson et al. 2013), collisions with windows in buildings (Machtans et al. 2013), collisions with power transmission lines (Rioux et al. 2013), mechanical agricultural activities such as haying or mowing, cultivation, and harvest (Tews et al. 2013), terrestrial oil and gas (Van Wilgenburg et al. 2013), and wind power (Zimmerling et al. 2013). Estimates from communication towers appear elsewhere (Longcore et al. 2012). Reports on several other anthropogenic activities with more limited data are cited here as unpublished works (roadside maintenance: D. Abraham, D. Pickard, and C. Wedeles, unpublished manuscript; agricultural pesticides: P. Mineau, unpublished manuscript; mining: J. Williams, unpublished manuscript; electrical and hydro power generation: J.-P. L. Savard and S. Rioux, unpublished manuscript; Appendix 1). Unless otherwise specified, the information for each source presented in this synthesis is drawn directly from these papers and reports.

Published mortality estimates for three other activities are also presented for comparison. Sport-hunting totals for migratory game birds in Canada from years 2000-2011 were obtained from the National Harvest Survey data base (http://www.cwsscf.ec.gc.ca/harvest-prises/def_e.cfm). Data on total annual harvest of nonmigratory game birds, mainly Galliformes, were obtained from provincial and territorial government web sites and representatives. We also include an estimate of seabird mortality from chronic ship-source oil pollution in the northwest Atlantic from the late 1990s (Wiese and Robertson 2004).

We were unable to include several additional sources of human-related mortality that may be important to Canadian bird populations. A recent assessment of livestock impacts (B. Bleho, N. Koper, and C. S. Machtans, unpublished manuscript) found both positive effects of vegetation management and negative effects of trampling on bird nests, estimating a loss of $\sim 1.5 \%$ of nests at a local scale, but is not included here because it did not quantify total mortality. We also did not calculate mortality and nest destruction from forest harvesting on private lands. Canada's National Forestry Database (http://nfdp.ccfm.org) indicates that private land harvest accounts for $\sim 19 \%$ of the total annual volume of wood harvested from all lands in Canada, but we did not assess whether harvest timing or bird densities were similar to those calculated for commercial harvest. We found little published information on the magnitude of avian mortality in Canada from aircraft-strikes, and impacts from large-scale tailings ponds remain uncertain (Timoney and Ronconi 2010), although the number of birds killed annually by these sources is expected to be small. Recent evidence also indicates potentially important population-level effects of rodenticides on birds of prey (Thomas et al. 2011), but this source of mortality was not considered here. Effects of the aquaculture industry were initially assessed because entanglements with exclusion nets or nets associated with farms are potential sources of mortality (Price and Nickum 1995). However, this mortality source has not been documented in Canada, and the consensus was that aquaculture currently causes very limited direct bird mortality. Information on indirect impacts of aquaculture development on marine bird populations is also limited, and shellfish aquaculture may sometimes benefit certain waterfowl species (Zydelis et al. 2006, 2009). As a result, aquaculture is not considered further. Finally, we do not include estimates of bird bycatch in freshwater fisheries although the documentation of large kills suggests this is an important information gap (e.g., Ellarson 1956).

\section{Comparing mortality estimates between sources}

Human activities can affect birds at different stages of their annual cycles. Activities that alter habitat during the breeding season, such as forestry and agricultural mowing, tend to destroy nests, eggs and young. Many other sources cause direct mortality of breeding adults, subadults, and juvenile birds, such as fishing or collisions with cars or buildings. We present total mortality estimates by the life stage where it occurs, to highlight differences among sources.

We used the methodology of Hobson et al. (2013) and Van Wilgenburg et al. (2013) to develop a stochastic simulation model that expresses stage-specific losses as an equivalent 
loss of potential adult breeders. This enabled a comparison of the effects of mortality affecting species at different life stages. In addition to allowing comparison of mortality across sources, this model explicitly quantified and combined the various sources of uncertainty in current mortality estimates. An advantage of this modeling approach is that it allowed us to combine data with various measures of central tendency and spread (means, medians, min-max ranges, confidence limits). These modeled values were also used to assess population-level effects of mortality.

The stochastic model controlled both for effects at differing life stages and for variation in life history strategies by converting all individuals to the potential breeding adult stage. However, we were unable to control for variation in time needed to reach those stages because longer lived and lowfecundity species take longer to reach breeding age, making populations slower to recover from perturbations. Our analysis also did not assess the effects of activities reducing future productivity through habitat loss or alteration, e.g., unreclaimed oil and gas clearings in forest, which may be a significant consequence of some of the industrial activities considered here. Our analysis does enable direct comparisons of mortality across various sources, which should be most reliable when focused on comparisons of sources that affect groups of species with similar life history characteristics. Most importantly, these comparisons of numbers killed do not take into account differences in population sizes of species, or species groups.

\section{Stochastic model to derive estimate of potential adult breeders killed}

Converting estimates of stage-specific losses to potential adult breeders using the stochastic model involved the following steps. First, we compiled estimates of stage-specific mortality (nest, egg/nestling, or independent bird) for each mortality source, including any information on age-composition (for independent birds killed) and species-group composition of the kill (see Appendix 2 for details). Additional author feedback was sought for some sources, especially regarding estimates of approximate species-group or age composition of the kill.

Next, unless exact values were available, probability distributions were assigned to all values for stage-specific kill totals, age-ratios, and species-group composition (see Appendix 2, Table A2.1). Kill totals from individual papers generally included some measure of central tendency (mean, median, or midpoint) and data spread (confidence interval or min-max range) that were converted to values required to model a log-normal distribution (mean $\mu$ and standard deviation $\sigma$ ). We modeled kill estimates as log-normal distributions because these estimates were all based on some multiplicative extrapolation. Age-ratios were modeled in various ways; draws from a binomial distribution were used when proportions were reasonably well known, beta distributions were used when estimated variances in proportions were available, and uniform distributions were used when only minimum and maximum values were reported. Similar distributions were used for species-group proportions, except that multinomial distributions were used when more than two species-groups were affected. For sport harvest of migratory birds, detailed data on age-ratios of the kill were available for ducks, geese, and shorebirds (snipe and woodcock), and age-ratio data for snipe and woodcock were applied to other species (doves, pigeons, rails, and cranes). Age-ratios were not needed for the harvest for upland nonmigratory game birds (Galliformes), because juvenile and adult nonbreeding season survivorship probabilities are comparable for these birds. Age at first breeding was assumed to be the second year of life for all species groups except seabirds, which were assumed to breed in their fifth year.

Demographic rates, with associated measures of data spread where available, were collated for each species group; these included clutch size, nest success, hatchability (or hatch success), survival of young to fledging, overwinter survivorship of juveniles, and adult survivorship. Note that in some instances only the product of several parameters was available, e.g., a general productivity value that equaled clutch size $\times$ hatching success $\times$ survival of hatchlings to fledgling (see Appendix 2, Table A2.2). For landbirds, except nonmigratory game birds, we used the values already collated in Hobson et al. (2013), with adult survival rates obtained from Johnston et al. (1997). All other demographic rates were obtained from literature values for species considered representative of each species group (Appendix 2, Table A2.2). For shorebirds, we chose values from two larger bodied upland nesting species, as these species are more likely to be affected by the mortality sources considered, i.e., mowing and collisions, compared to smaller Arctic-breeding migrants. When a particular value was not available, notably overwinter survival of hatch-year birds $\left(S_{o}\right)$, this value was estimated using the other vital rates available, assuming a stable population $\left(S_{o}=\left(1-S_{a}\right) / F\right)$, where $S_{a}$ is adult survival and $F$ is fecundity (number of independent young produced). A variety of distributions was used to model these vital rates. For instance, beta distributions were used for well-estimated parameters, draws from uniform distributions were used when uncertainty was high and only minimum and maximum values were available, and random draws from a collection of rates were used for landbirds and shorebirds where a number of estimates were available. See Appendix 2 for additional details on vital rates used for each species group.

Finally, these values and distributions were used to estimate the equivalent number of potential adult breeders that would be removed from the population, based on the stage-specific kill estimates. For example, for an activity that kills eggs and nestlings at the start of the breeding season, draws from the 
distribution of total kill of eggs for a given species group were multiplied by draws for estimates of nest success, hatch success, survival of young to fledging, and overwinter survival for that species group. Models were run 100,000 times, and various descriptive statistics of the resulting distributions were extracted. We present medians with $90 \%$ intervals, to allow direct comparison of the numbers presented for forestry (Hobson et al. 2013) and terrestrial oil and gas (Van Wilgenburg et al. 2013). Note that no conversion was necessary for these two sectors because the authors directly converted their estimates of nest losses to the equivalent loss of potential adult breeders.

\section{Extent, scale, and scope of mortality}

We tabulated the season when most human-related mortality occurs (spring, breeding, fall, winter) in Canada for each of the main groups (landbirds, seabirds, shorebirds, waterbirds, waterfowl) to better understand the timing and extent of mortality across Canadian bird populations. We assigned a qualitative score of 'no/little known effect,' 'some effect,' or 'large effect' to each source/group/season combination, based on the information in each paper or report and feedback from their authors. Generally, a 'large effect' score was assigned when a particular species group was clearly identified as being frequently killed during a given season, whereas 'some effect' was assigned to species groups and seasons that were peripherally affected. Note that factors that kill birds while they are outside of Canada, including human-caused mortality to migrants, were not included in this assessment.

To quantify the relative population impact of differing sources of human-related mortality (hereafter 'population-level impacts'), we compared the estimated mortality to the total abundance of individual populations, species, or families where data were available at that resolution; in some cases, mortality data were not available below the level of broad taxonomic group. For wind power, marine industries, oil and gas, agriculture, and roadside maintenance, we present population-level impacts that were directly calculated by the paper or report authors; for building collisions, we calculated family-level impacts by combining kill data provided by authors with current estimates of family-level abundance in Canada (Blancher 2002; P. Blancher unpublished data). For all these estimates, total kill of nests/eggs/nestlings was converted to the equivalent mortality of potential breeding adults, as described above, to enable comparability among sources of mortality; see Appendix 3 for full details on population-level kill and abundance. Note that although population-level impact estimates provide examples of the relative importance of particular mortality sources, these populations do not represent a random sample of all population-level impacts because they may have been highlighted by authors for different reasons, e.g., those considered particularly at risk, those representative of most birds affected, or those with the best available data on population size. We considered reference levels of $10 \%, 1 \%$, and $0.1 \%$ to be informative. Individual sectors near or above $10 \%$ could likely translate to detectable negative population effects. Population proportions of $1 \%$ are considered nationally significant from the perspective of management of protected areas (e.g., RAMSAR criteria). We are not aware of documented population effects for rates of mortality below $0.1 \%$ from individual sources.

\section{Spatial assessment of mortality risk}

A spatial representation of cumulative human-related mortality in Canada was created for a subset of sectors. Applicable or proxy spatial information was available for the following eight sources of terrestrial-based mortality: cats, bird-window collisions, bird-vehicle collisions, birdcommunication tower collisions, agriculture (haying and crops), commercial forestry, oil and gas, and wind turbines. All data were summarized and displayed on a $50 \times 50 \mathrm{~km}$ tile grid covering Canada. This grid-level balanced the goal of providing interpretable images against the false precision of mapping data that usually had low spatial resolution or concordance with specific processes causing mortality, e.g., we know precisely where all paved roads are, but not where bird-vehicle collisions occur on those roads. All data sources and detailed procedures used to derive the maps are provided in Appendix 4.

We began by taking the proportion of activity in a $50 \times 50 \mathrm{~km}$ tile grid across areas of resolution defined by the original research paper, e.g., provincially for forestry; by turbine for wind facilities; and by applicable portions of Bird Conservation Regions for agriculture. The total mortality estimate for each tile was then calculated by multiplying the proportion of activity in each tile by the original mortality estimate (number of wind turbines, $\mathrm{km}^{2}$ of oil and gas activity, etc.). The completed tiles from the eight sources were overlaid and summed to compute the total mortality estimate per tile.

The final map was colored using 10 classes calculated by the Jenks classifier (Jenks 1967) in ArcGIS 10 and output in raster format. We applied a low-pass filter to the raster output using a $5 \times 5$ tile kernel size (Jensen 2005). We caution that the map represents an index of probable mortality across key sources, and is only an approximation. Accurately mapping mortality would require spatially explicit information on bird density, specific details on how and when each sector interacts with birds in each tile, and a variety of covariates that are not available nationally or may not be understood, e.g., why does mortality at tall buildings apparently differ appreciably among cities (Machtans et al. 2013)?

\section{RESULTS}

\section{Total mortality estimates}

Mortality estimates from each human-related source ranged from a few thousand to tens or hundreds of millions of birds. 
In Canada, all combined sources of human-related mortality destroyed an average of $\sim 2$ million nests and killed $\sim 269$ million birds per year, or the equivalent of $\sim 186$ million potential adult breeders each year (Fig. 1). Cats and collisions with structures were the largest causes of human-related bird mortality in Canada: cumulatively, the top five sources of mortality, i.e., predation by feral and pet cats, and collisions with road vehicles, houses, and transmission lines, represented more than $95 \%$ of the individuals killed across all humanrelated sources. Because each of these top-ranking mortality sources are widespread, they may represent relatively small numbers at the local scale, but sum to very high levels of mortality when extrapolated across Canada. In contrast, some other mortality sources do not occur uniformly across the country, e.g., terrestrial oil and gas, fisheries, or are from industries located at relatively few scattered locations, e.g. wind power, and thus have relatively modest national-level kill totals, despite measurable localized effects.

The nine largest sources of anthropogenic mortality all killed mobile individual birds, including adult, subadult, and juvenile birds, although over a million nests and eggs are destroyed annually by forestry and agriculture, respectively (Fig. 1A). Fig. 1A and Table 1 show the total number killed by each source, identifying the life stage at which most mortality occurs, i.e., nest destruction, mortality of eggs or nestlings, or loss of independent mobile individuals. Mortality occurring at two stages, i.e., loss of eggs and mobile individuals through road maintenance, is shown as two points for that source. Note that although most estimates were made at a national level, for example, by extrapolating from local-scale estimates across the country, a few were only made at smaller scales (indicated as hollow symbols in Fig. 1): the agricultural haying and road maintenance estimates each represent impacts on just five and six focal species, respectively, and the hydro reservoir estimate was made for Quebec only. Total Canada-wide crosstaxa mortality caused by these activities is therefore likely to be appreciably higher than the values presented here.

The relative ranking of mortality sources was similar for the stage-specific and converted values (Figs. 1A, 1B), particularly for the largest sources of mortality. However, for human activities that destroy eggs and nests, the equivalent potential adult breeder total was considerably reduced, and thus the relative ranking of these sources somewhat altered, because many of the eggs or young killed by these sources would have not been expected to survive to adulthood otherwise (Fig 1B).

Converted estimates pooled across related activities provided broad estimates for the main sources of human-caused mortality (Fig 1C). These pooled sectors were cats (feral and pet), transportation (vehicle-collisions, road maintenance, and chronic ship-source oil), buildings (collisions with all three types), electrical power (transmission-line collisions, hydro reservoirs, electrocutions, transmission-line maintenance, and wind energy), harvest (migratory and nonmigratory game birds), agriculture (haying and pesticides), fisheries (all gear types), oil and gas (all terrestrial and marine sources), and mining (pits/quarries and metals/minerals); the original single-source values for forestry and communication towers are also shown. Nonindustrial activities (cats, transportation, and buildings) still represented the greatest overall sources of mortality, while electrical power and agriculture represented the largest industrial sources of mortality, with an annual kill of over 18 million and over 2 million potentially breeding birds, respectively. At the other end of the spectrum, the fisheries, oil and gas, and mining industries each killed the equivalent of fewer than 25,000 breeders annually (Fig. 1C). Note that within sectors, some sources of mortality were relatively low, e.g., electrocutions in the electrical power sector, while others dominated the overall sectoral kill, e.g., transmission line collisions.

\section{Evaluating potential population effects: seasonal and taxonomic distribution of mortality}

The distribution of anthropogenic mortality among bird groups and across seasons for each mortality source showed that landbirds as a group were affected by the widest range of human activities (Table 2). These impacts occurred primarily during the breeding seasons, as expected, because many species overwinter outside of Canada. Shorebirds and waterfowl also faced many potential threats at their nesting sites, and birds across all groups confronted a range of humancaused mortality during spring and fall migration, particularly from collisions with cars, buildings, power-lines, and transmission structures.

Landbirds make up the majority of all Canadian breeding birds, and they constituted most of the estimated total mortality among the five species groups when expressed in common units of potential adult breeders (Table 3). In total, we estimated that $89 \%$ of all birds killed annually by human activities are landbirds; $6 \%$ are waterfowl, and the remaining $5 \%$ includes waterbirds, shorebirds, and seabirds. The majority of mortality occurred through direct kill of mobile individuals (74\%; mostly cats, but see Table 2 for categories of impact type), with $25 \%$ of mortality caused by collisions. The destruction of nests represented less than $1 \%$ of overall estimated impact when converted to potential adult breeders.

Although overall national-scale mortality estimates illustrated the magnitude of bird mortality across Canada, some humanrelated activities had disproportionately large effects on particular species or populations, with the potential for population-level impacts at a regional or national level (Fig. 2; see Appendix 3 for full details). For example, marine fisheries bycatch had one of the lowest total mortality estimates nation-wide, but may annually kill a relatively large 
Fig. 1. Annual mortality of Canadian birds due to human activities (log-scale). Panel A shows stage-specific estimates for each activity, according to whether entire nests, single eggs/nestlings, or mobile individuals were killed, as in original papers and reports. Values include both means and medians, and error bars represent both confidence limits (90\% or 95\%) and maximum/minimum ranges, as originally presented. Panel B shows converted mortality estimates for each activity (median with $90 \%$ confidence limits), where stage-specific kill totals have been converted to the equivalent number of potential adult breeders based on a stochastic model incorporating species-composition and demography. Hollow symbols indicate mortality only estimated for part of Canada or for a limited number of species, and thus where total Canada-wide cross-taxa mortality is likely much higher than these estimates. Panel C shows these same converted estimates (median with $90 \%$ confidence limits), pooled across related activities (cats: feral and pet; transportation: vehicle-collisions, road maintenance, and chronic ship-source oil; buildings: collisions with all 3 types; power: transmission-line collisions, hydro reservoirs, electrocutions, transmission-line maintenance, and wind energy; agriculture: haying and pesticides; harvest: migratory and nonmigratory birds; fisheries: all gear types; oil and gas: all terrestrial and marine sources; mining: both pits/quarries and metals/minerals), as well as the original single-source values for forestry and communication towers. Values in all panels are ranked in descending order according to the converted kill totals. See text and Appendix 2 for citations of papers and reports used as data sources.

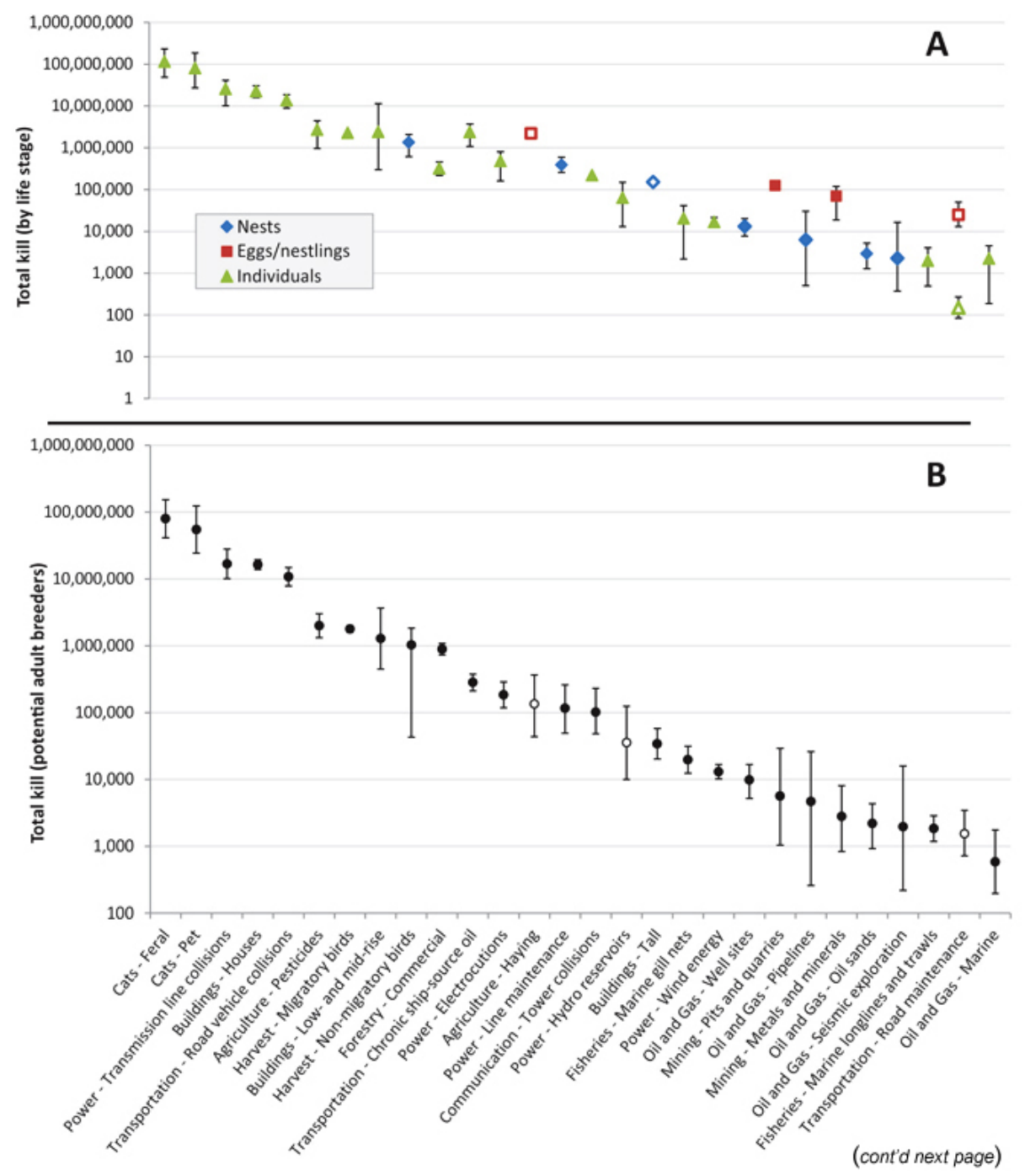


Figure 1 continued.

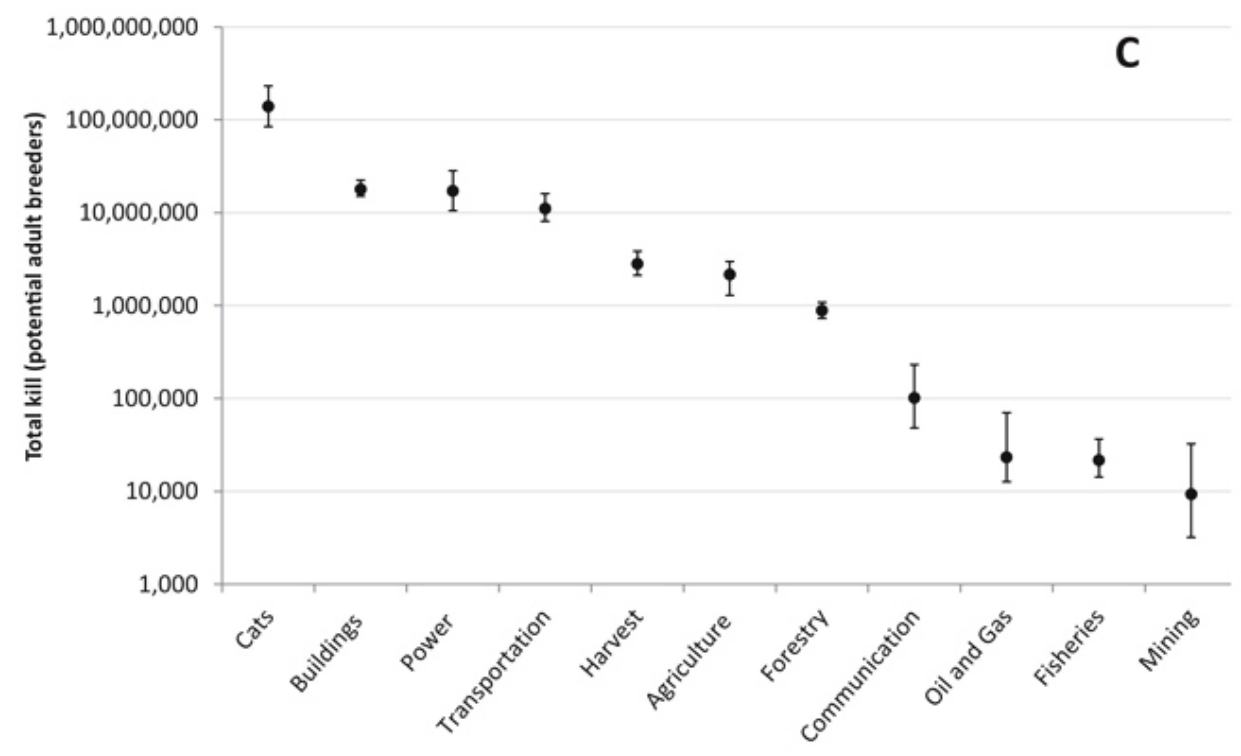

proportion of Canadian populations of a few species, e.g., Black-footed Albatross Phoebastria nigripes: $4 \%$ of the entire Canadian population, or Common Eiders Somateria mollissima: $7 \%$ of the Nova Scotia breeding population (Fig. 2). Mortality from building collisions also nonrandomly impacted landbirds. Overall, tall buildings killed less than $0.01 \%$ of total abundance of any landbird family, whereas between $2-5 \%$ of nuthatches, chickadees, and pigeons may have been killed at houses (see Bayne et al. 2012 for proportions of house-collision kills by family, which we used in Appendix 3 and Fig. 2). Although this simple comparison does not capture the complexity of potential population effects, it confirms that national mortality totals alone do not reflect the ecological importance of human-related activities for most species and that mortality is not simply proportional to abundance (see also Longcore et al. 2013).

We did not directly assess the impacts of sport harvest on populations of game birds because ongoing assessments exist elsewhere (e.g. Williams and Johnson 1995, Nichols et al. 2007), and extensive programs are in place throughout North America that ensure that any population-level effects of regulated harvests are sustainable in the long term (e.g., Runge et al. 2009). These impacts would likely have dominated Fig. 2 , because sport-harvest was clearly important as a humanrelated source of mortality in Canada for waterfowl and an important factor for some other bird groups (Table 3).

\section{Spatial distribution of mortality risk and potential cumulative effects}

Human-related mortality from terrestrial sources was not uniformly distributed across Canada (Fig. 3A) because areas of higher mortality corresponded with areas of high human population and high human activity. Peak mortality for all sources combined was highest in southern Ontario and Quebec, around the five major prairie cities, and in southwestern British Columbia. In addition to having high human populations, and correspondingly large numbers of cats, buildings, and roads, numerous industries overlap with these areas. Overall, very little avian mortality from the sources that we mapped currently occurs in the northern part of many provinces and in the territories.

The distribution of mortality when excluding the three largest sources (cats, buildings, roads) was spread more evenly across southern Canada (Fig. 3B), partly reflecting broad areas of forest harvesting and the diffuse distribution of communication towers across this area. Southern Alberta and southeastern Ontario appeared to be areas for potential additive effects of multiple industries. The high values in the Maritimes were partially attributable to forestry, whereas those in the lower mainland of British Columbia primarily reflect the high number of hay farms. Individual, unsmoothed maps for each mortality source are provided in Appendix 4.

In contrast to most impacts of clearing activities (Fig. 3B), collision-based sources of mortality impacted some species more than others, and thus potential cumulative effects were harder to assess spatially. Based on available data, we found indications that different types of collisions appeared to affect different groups of landbirds. At the family level, warblers dominated birds killed in communication tower collisions (15 of the most abundant 20 species recorded, Longcore et al. 2013) whereas a wider variety of species dominated tall 
Table 1. Life stage-specific (nests, eggs/ nestlings, or independent individuals) mortality estimates of human-related avian mortality in Canada derived directly from published papers and unpublished reports. These values are illustrated in Fig. 1A, and served as the basis for the stochastic model conversion to an equivalent number of potential adult breeders; mortality sources are listed in descending order of converted kill totals. Characteristics of the estimate are indicated in the last column, i.e., whether central values were mean, median, or midpoint of a range, and whether lower/upper values represent a confidence interval (CI) or a range. Note that the estimates for forestry and terrestrial oil and gas shown here represent the estimated number of nests destroyed.

\begin{tabular}{|c|c|c|c|c|c|c|c|c|c|c|}
\hline \multirow[b]{2}{*}{ Source } & \multicolumn{3}{|c|}{ Nests } & \multicolumn{3}{|c|}{ Eggs or Nestlings } & \multicolumn{3}{|c|}{ Individuals } & \multirow{2}{*}{$\begin{array}{c}\text { Values } \\
\text { Estimated }\end{array}$} \\
\hline & Lower & Central & Upper & Lower & Central & Upper & Lower & Central & Upper & \\
\hline Cats - Feral & & & & & & & $49,000,000$ & $116,000,000$ & $232,000,000$ & $\begin{array}{l}\text { median, } \\
95 \% \mathrm{CI}\end{array}$ \\
\hline Cats - Domestic & & & & & & & $27,000,000$ & $80,000,000$ & $186,000,000$ & $\begin{array}{l}\text { median, } \\
95 \% \mathrm{CI}\end{array}$ \\
\hline Power - Transmission line collisions & & & & & & & $10,100,000$ & $25,600,000$ & $41,200,000$ & $\begin{array}{l}\text { mean, } \\
95 \% \text { CI }\end{array}$ \\
\hline Buildings - Houses & & & & & & & $15,800,000$ & $22,400,000$ & $30,500,000$ & $\begin{array}{l}\text { mean, } \\
\text { range }\end{array}$ \\
\hline $\begin{array}{l}\text { Transportation - Road vehicle } \\
\text { collisions }\end{array}$ & & & & & & & $8,914,341$ & $13,810,906$ & $18,707,470$ & $\begin{array}{l}\text { mean, } \\
95 \% \text { CI }\end{array}$ \\
\hline Agriculture - Pesticides & & & & & & & 960,011 & $2,695,415$ & $4,430,819$ & $\begin{array}{l}\text { midpoint, } \\
\text { range }\end{array}$ \\
\hline Harvest - Migratory birds & & & & & & & & $2,279,655$ & & mean \\
\hline Buildings - Low- and midrise & & & & & & & 300,000 & $2,400,000$ & $11,400,000$ & $\begin{array}{l}\text { mean, } \\
\text { range }\end{array}$ \\
\hline Harvest - Nonmigratory birds & & & & & & & $1,076,810$ & $2,389,124$ & $3,701,438$ & $\begin{array}{l}\text { mean, } \\
95 \% \mathrm{CI}\end{array}$ \\
\hline Forestry - Commercial & 615,959 & $1,351,340$ & $2,086,720$ & & & & & & & $\begin{array}{l}\text { midpoint, } \\
\text { range }\end{array}$ \\
\hline $\begin{array}{l}\text { Transportation - Chronic ship-source } \\
\text { oil }\end{array}$ & & & & & & & 217,800 & 321,900 & 458,600 & $\begin{array}{l}\text { mean, } \\
95 \% \mathrm{CI}\end{array}$ \\
\hline Power - Electrocutions & & & & & & & 160,836 & 481,399 & 801,962 & $\begin{array}{l}\text { midpoint, } \\
\text { range }\end{array}$ \\
\hline Agriculture - Haying & & & & & $2,209,400$ & & & & & mean \\
\hline Power - Line maintenance & 258,849 & 388,274 & 592,418 & & & & & & & $\begin{array}{l}\text { midpoint, } \\
\text { range }\end{array}$ \\
\hline Communication - Tower collisions & & & & & & & & 220,649 & & mean \\
\hline Power - Hydro reservoirs & & 152,162 & & & & & & & & mean \\
\hline Buildings - Tall & & & & & & & 13,000 & 64,000 & 149,000 & $\begin{array}{l}\text { mean, } \\
\text { range }\end{array}$ \\
\hline Fisheries - Marine gill nets & & & & & & & 2185 & 20,612 & 41,528 & $\begin{array}{l}\text { mean, } \\
\text { range }\end{array}$ \\
\hline Power - Wind energy & & & & & & & 13,330 & 16,700 & 21,600 & $\begin{array}{l}\text { mean, } \\
95 \% \text { CI }\end{array}$ \\
\hline Oil and Gas - Well sites & 7688 & 13,182 & 20,249 & & & & & & & $\begin{array}{l}\text { median, } \\
90 \% \mathrm{CI}\end{array}$ \\
\hline Mining - Pits and quarries & & & & & 125,529 & & & & & mean \\
\hline Oil and Gas - Pipelines & 503 & 6314 & 30,234 & & & & & & & $\begin{array}{l}\text { median, } \\
90 \% \mathrm{CI}\end{array}$ \\
\hline Mining - Metals and minerals & & & & 18,653 & 69,211 & 119,768 & & & & $\begin{array}{l}\text { midpoint, } \\
\text { range }\end{array}$ \\
\hline Oil and Gas - Oil sands & 1281 & 2939 & 5236 & & & & & & & $\begin{array}{l}\text { median, } \\
90 \% \mathrm{CI}\end{array}$ \\
\hline Oil and Gas - Seismic exploration & 374 & 2280 & 16,438 & & & & & & & $\begin{array}{l}\text { median, } \\
\text { range }\end{array}$ \\
\hline Fisheries - Marine longlines and trawls & & & & & & & 494 & 1,999 & 4058 & $\begin{array}{l}\text { mean, } \\
\text { range }\end{array}$ \\
\hline Transportation - Road maintenance & & & & 13,086 & 25,149 & 50,294 & 84 & 149 & 270 & $\begin{array}{l}\text { median, } \\
\text { range }\end{array}$ \\
\hline Oil and Gas - Marine & & & & & & & 188 & 2244 & 4494 & $\begin{array}{l}\text { median, } \\
\text { range }\end{array}$ \\
\hline TOTAL & & $1,916,491$ & & & $2,429,289$ & & & $268,704,752$ & & \\
\hline
\end{tabular}


Fig. 2. Proportion of population affected by anthropogenic mortality on Canadian birds, by species group (panel A) and by mortality source (panel B), for populations where data were available at sufficient resolution. Estimated annual kill for a given species, population, or family (converted to potential adult breeders) is plotted against the estimated Canadian abundance for that group, to show the estimated proportion of the total population killed by each activity. The three diagonal lines represent a mortality rate of $10 \%, 1 \%$, and $0.1 \%$ for visual reference and are explained in more detail in the text. Details of mortality and abundance totals, as well as the identity of the species/population/family represented by each data point, are provided in Appendix 3. Game bird harvests are not included in this figure because they would dominate the figure and this source of mortality is regulated.
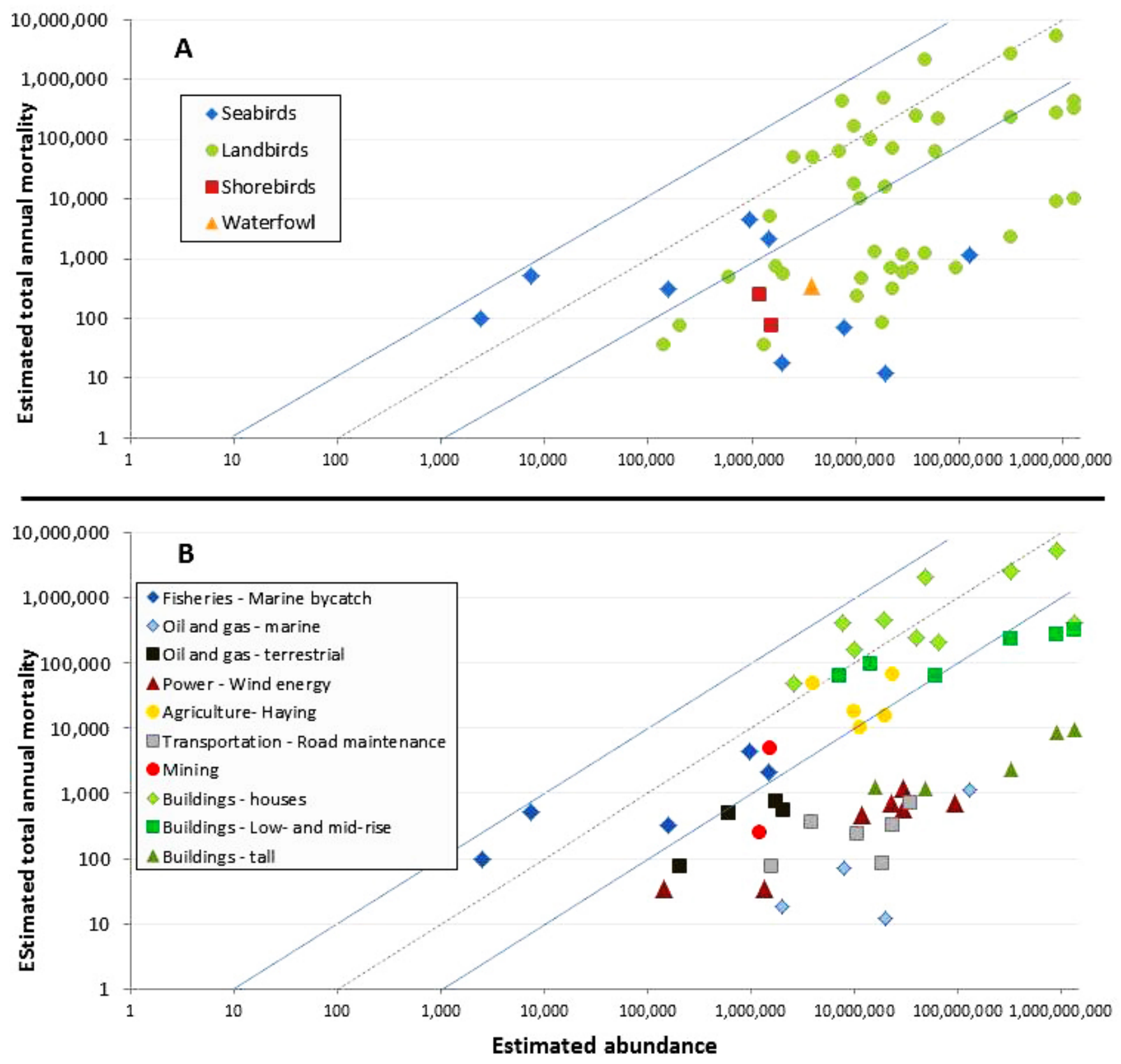
Fig. 3. Approximated distribution of total bird mortality estimates in Canada from eight terrestrial sources (cats, building collisions, vehicle collisions, agriculture, forestry, terrestrial oil and gas, communication towers, and wind turbines). Panel A is the sum of all eight sources, while panel B excludes the first three in the above list. These maps present the probability of mortality based on the distribution of each source in Canada. The hotspot on Montreal is because a single tile of our grid overlapped that city perfectly, while, for example, Toronto was centered at the intersection of 4 tiles. Unsmoothed maps for each mortality source and all mapping methods are provided in Appendix 4.

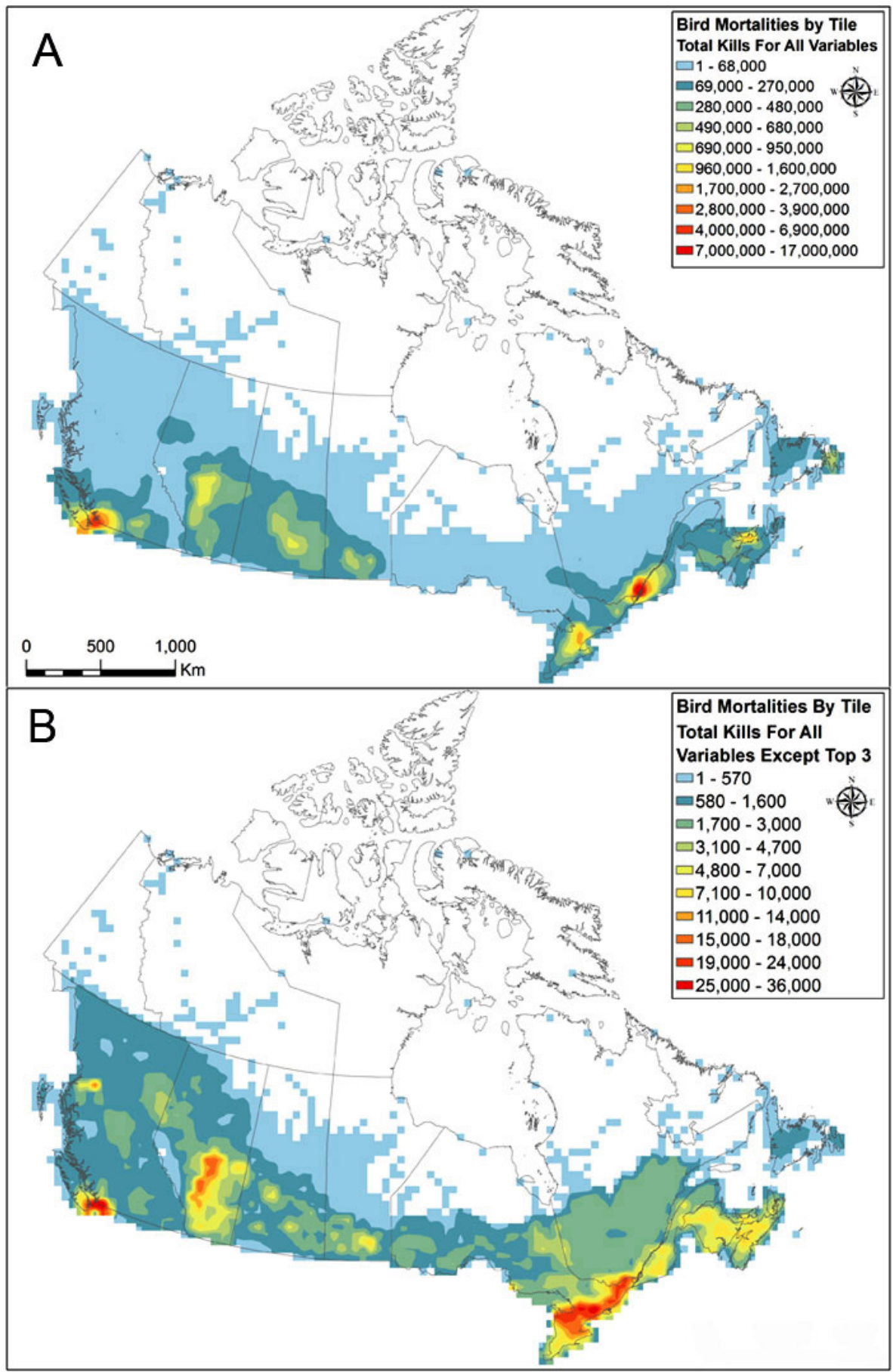


Table 2. Seasonal and species-group breakdown for each source of human-related avian mortality in Canada: o little or no known effect, + some effect, including effects anticipated but not quantified [highlighted yellow], ++ large effect [highlighted orange], na not applicable. Within the effect-type categories (collisions, direct kill, or nest destruction), mortality sources are ordered in descending order of converted kill totals, as presented in Fig. 1B. Comparisons should be made within source rows, rather than within columns because the level of effect was evaluated qualitatively among seasons and species-groups within each source, and is not intended to reflect differences in magnitude among sources. Note that 'winter' refers only to impacts on birds while wintering in Canada.

\begin{tabular}{|c|c|c|c|c|c|c|c|c|c|c|c|c|c|c|c|c|c|c|c|c|c|}
\hline \multirow[b]{2}{*}{$\begin{array}{l}\text { Primary type of } \\
\text { impact }\end{array}$} & \multirow[b]{2}{*}{ Source } & \multicolumn{4}{|c|}{ LANDBIRDS } & \multicolumn{4}{|c|}{ SEABIRDS } & \multicolumn{4}{|c|}{ SHOREBIRDS } & \multicolumn{4}{|c|}{ WATERBIRDS } & \multicolumn{4}{|c|}{ WATERFOWL } \\
\hline & & $\begin{array}{l}\text { S- } \\
\text { PR }\end{array}$ & $\begin{array}{l}\text { B- } \\
\text { RE }\end{array}$ & $\begin{array}{l}\text { F- } \\
\text { A- } \\
\text { LL }\end{array}$ & WIN & $\begin{array}{l}\text { NS- } \\
\text { PR }\end{array}$ & $\begin{array}{l}\mathrm{B}- \\
\mathrm{RE}\end{array}$ & $\begin{array}{l}\text { F- } \\
\text { A- } \\
\text { LL }\end{array}$ & & $\begin{array}{l}\text { NS- } \\
\text { PR }\end{array}$ & $\begin{array}{l}\mathrm{B}- \\
\mathrm{RE}\end{array}$ & $\begin{array}{l}\text { F- } \\
\text { A- } \\
\text { LL }\end{array}$ & & $\begin{array}{l}\text { JS- } \\
\text { PR }\end{array}$ & $\begin{array}{l}\mathrm{B}- \\
\mathrm{RE}\end{array}$ & $\begin{array}{l}\text { F- } \\
\text { A- } \\
\text { LL }\end{array}$ & & $\begin{array}{l}\text { NS- } \\
\text { PR }\end{array}$ & $\begin{array}{l}\mathrm{B}- \\
\mathrm{RE}\end{array}$ & $\begin{array}{l}\text { F- } \\
\text { A- } \\
\text { LL }\end{array}$ & WIN \\
\hline \multirow[t]{8}{*}{ Collisions } & $\begin{array}{l}\text { Transportation - Road vehicle } \\
\text { collisions }\end{array}$ & + & ++ & + & + & o & o & o & o & + & + & + & o & + & + & + & o & + & + & + & o \\
\hline & Buildings - Houses & ++ & ++ & ++ & + & o & o & o & o & o & o & o & o & $\mathrm{o}$ & o & o & o & o & o & o & o \\
\hline & Power - Transmission line collisions & + & + & + & + & o & o & o & o & ++ & ++ & ++ & + & + & + & + & + & ++ & + & ++ & + \\
\hline & Buildings - Low- and mid-rise & ++ & ++ & ++ & $\mathrm{o}$ & o & $\mathrm{o}$ & o & o & o & $\mathrm{o}$ & o & o & o & o & o & o & o & o & o & o \\
\hline & Power - Electrocutions & + & + & + & + & o & o & o & o & + & o & + & o & + & o & + & o & $\mathrm{o}$ & o & $\mathrm{o}$ & o \\
\hline & Communication - Tower collisions & ++ & + & ++ & + & o & o & o & o & + & o & + & o & + & o & + & o & + & o & + & o \\
\hline & Buildings - Tall & ++ & o & ++ & o & o & o & o & o & o & o & o & o & o & o & o & o & o & o & o & o \\
\hline & Power - Wind energy & + & ++ & + & o & o & o & o & o & o & o & o & o & o & o & o & o & o & o & o & o \\
\hline \multirow[t]{8}{*}{ Direct kill } & Cats (feral and domestic) & ++ & ++ & ++ & ++ & o & o & o & o & o & + & o & o & o & + & o & o & o & + & o & o \\
\hline & Agriculture - Pesticides & + & ++ & + & o & o & o & o & o & + & + & + & o & + & + & + & o & + & + & + & o \\
\hline & Harvest - Migratory game birds & o & o & o & o & o & o & + & + & o & o & + & + & o & o & + & + & + & o & ++ & + \\
\hline & Harvest - Non-migratory game birds & o & $\mathrm{o}$ & ++ & + & o & o & o & o & o & o & o & o & o & o & o & o & o & o & o & o \\
\hline & $\begin{array}{l}\text { Transportation - Chronic ship-source } \\
\text { oil }\end{array}$ & o & o & o & o & o & o & o & ++ & o & o & o & o & o & o & o & o & o & o & o & o \\
\hline & Fisheries - Marine gillnets & o & o & o & o & o & ++ & + & o & o & o & o & o & o & o & o & o & o & + & o & o \\
\hline & $\begin{array}{l}\text { Fisheries - Marine longlines and } \\
\text { trawls }\end{array}$ & o & o & o & o & o & + & + & o & o & o & o & o & $\mathrm{o}$ & o & o & o & o & o & o & o \\
\hline & Oil and Gas - Marine ${ }^{\dagger}$ & o & o & $\mathrm{o}$ & $\mathrm{o}$ & + & + & + & + & o & o & o & o & o & o & o & o & o & o & o & o \\
\hline \multirow[t]{7}{*}{ Nest destruction } & Agriculture - Haying and mowing & na & ++ & na & na & na & o & na & na & na & o & na & na & na & o & na & na & na & ++ & na & na \\
\hline & Forestry - Commercial & na & ++ & na & na & na & o & na & na & na & + & na & na & na & o & na & na & na & + & na & na \\
\hline & Power - Line maintenance & na & ++ & na & na & na & o & na & na & na & ++ & na & na & na & ++ & na & na & na & ++ & na & na \\
\hline & Power - Hydro reservoirs & na & ++ & na & na & na & o & na & na & na & ++ & na & na & na & ++ & na & na & na & ++ & na & na \\
\hline & Oil and Gas - Terrestrial (all) & na & ++ & na & na & na & o & na & na & na & + & na & na & na & o & na & na & na & + & na & na \\
\hline & Mining (all) & na & ++ & na & na & na & $\mathrm{o}$ & na & na & na & + & na & na & na & o & na & na & na & + & na & na \\
\hline & Transportation - Road maintenance ${ }^{\ddagger}$ & na & ++ & na & na & na & o & na & na & na & + & na & na & na & o & na & na & na & ++ & na & na \\
\hline
\end{tabular}

mortality from both direct kill and collisions;

* mortality from both nest destruction and direct kill

building collisions (only 6 of the top 20 were warblers, Machtans et al. 2013). At the species level, the top five species killed in tall building collisions in southern Ontario (based on the Toronto Fatal Light Awareness Program, www.flap.org) were Golden-crowned Kinglet (Regulus satrapa), Whitethroated Sparrow (Zonotrichia albicollis), Ruby-crowned Kinglet (Regulus calendula), Dark-eyed Junco (Junco hyemalis), and Ovenbird (Seiurus aurocapilla), together comprising $42 \%$ of mortalities. In contrast, the top five species killed in communication tower collisions in the Bird Conservation Region, which includes Toronto (Longcore et al. 2013), were Ovenbird, Ruby-crowned Kinglet, Blackpoll Warbler (Setophaga striata), Red-eyed Vireo (Vireo olivaceus), and Common Yellowthroat (Geothlypis trichas), together comprising $44 \%$ of mortalities. Species reported killed most often at wind-turbines only showed some overlap with these other collision-sources, with the top five being
Horned Lark (Eremophila alpestris), Golden-crowned Kinglet, Red-eyed Vireo, European Starling (Sturnus vulgaris), and Tree Swallow (Tachycineta bicolor; Zimmerling et al. 2013). Only 80\% of birds killed at wind turbines were passerines, proportionately much lower than at communication towers ( $97 \%$ passerines, Longcore et al. 2013) or in collisions with windows of tall buildings $(90 \%$ passerines, Machtans et al. 2013). Much better species-level data are required concerning cat kills and window collisions at homes, as well as from the range of other human activities for which population-level data are not yet available, to better understand the most significant population impacts and to identify additive or cumulative impacts. Even the species comparisons above should be taken with caution because the spatial scale of the data sources differ across each study. 
Table 3. Median annual estimates of human-related mortality in Canada across the five major species groups, based on a stochastic model that converted stage-specific mortality to potential adult breeders, ranked in descending order according to total estimated mortality across all bird groups. Note that species-group totals do not sum exactly to the 'all birds' value because uncertainty in species composition was explicitly modeled and the "all birds" value was modeled independently of each species group's total. See text and Appendix 2 for details of the stochastic model conversions. In cases where mortality was not fully extrapolated to all regions and taxa, e.g., where it was only estimated for a given region or set of focal species, the taxonomic or regional scope of the estimate is indicated; impacts estimated Canada-wide and across taxa are indicated as 'all' in the Scope column.

\begin{tabular}{|c|c|c|c|c|c|c|c|}
\hline SOURCE & SCOPE & LANDBIRDS & SEABIRDS & SHOREBIRDS & WATERBIRDS & WATERFOWL & ALL BIRDS \\
\hline Cats - Feral & All & $78,600,000$ & & & 293,400 & 380,500 & $79,600,000$ \\
\hline Cats - Domestic & All & $54,150,000$ & & & 199,300 & 258,300 & $54,880,000$ \\
\hline Power - Transmission line collisions & All & 574,700 & & $2,548,000$ & $5,170,000$ & $8,459,000$ & $16,810,000$ \\
\hline Buildings - Houses & All & $16,390,000$ & & & & & $16,390,000$ \\
\hline Transportation - Road vehicle collisions & All & $8,743,000$ & & 197,000 & 187,200 & 218,500 & $9,814,000$ \\
\hline Agriculture - Pesticides & All & $1,898,000$ & & 19,230 & 19,430 & 19,130 & $1,998,000$ \\
\hline Harvest - Migratory game birds & All & 235 & 55,520 & 24,770 & 8773 & $1,691,000$ & $1,786,000$ \\
\hline Buildings - Low- and mid-rise & All & $1,132,000$ & & 26,310 & 23,870 & 32,190 & $1,283,000$ \\
\hline Harvest - Non-migratory game birds & All & $1,031,000$ & & & & & $1,031,000$ \\
\hline Forestry - Commercial & Landbirds & 887,835 & & & & & 887,835 \\
\hline Transportation - Chronic ship-source oil & All & & 282,700 & & & & 282,700 \\
\hline Power - Electrocutions & All & 178,200 & & 1715 & 1854 & 2275 & 184,300 \\
\hline Agriculture - Haying and mowing & 5 species & 135,400 & & & & & 135,400 \\
\hline Power - Line maintenance & All & 70,140 & & 4474 & & 33,030 & 116,000 \\
\hline Communication - Tower collisions & All & 101,500 & & 965 & 1050 & 1278 & 101,500 \\
\hline Power - Hydro reservoirs & Québec & 31,260 & & 490 & 1571 & 158 & 35,770 \\
\hline Buildings - Tall & All & 32,000 & & 388 & 339 & 501 & 34,130 \\
\hline Fisheries - Marine gill nets & All & & 19,790 & & & & 19,790 \\
\hline Power - Wind energy & All & 13,060 & & & & & 13,060 \\
\hline Oil and Gas - Well sites & Landbirds & 9815 & & & & & 9815 \\
\hline Mining - Pits and quarries & All & 5169 & & 39 & 168 & & 5637 \\
\hline Oil and Gas - Pipelines & Landbirds & 4687 & & & & & 4687 \\
\hline Mining - Metals and minerals & All & 2798 & & & & & 2798 \\
\hline Oil and Gas - Oil sands & Landbirds & 2193 & & & & & 2193 \\
\hline Oil and Gas - Seismic exploration & Landbirds & 1966 & & & & & 1966 \\
\hline Fisheries - Marine longlines and trawls & All & & 1843 & & & & 1843 \\
\hline Transportation - Road maintenance & 6 species & 1103 & & 71 & & 324 & 1545 \\
\hline Oil and Gas - Marine & All & & 584 & & & & 584 \\
\hline TOTAL & & $163,980,226$ & 360,437 & $2,848,252$ & $5,931,455$ & $11,124,386$ & $186,429,553$ \\
\hline
\end{tabular}

\section{DISCUSSION}

\section{Interpreting mortality estimates}

Human-related activities inadvertently kill hundreds of millions of birds and destroy millions of nests in Canada every year, with landbirds most affected. Birds are primarily affected during the breeding season, although collisions occur year round. Landbirds were subject to the largest diversity of impacts, suggesting that they may be most vulnerable to additive effects across sources and seasons. Many of these human-related activities also pose a threat to migrants when outside of Canada, mortality that has not been quantified here, and thus the cumulative year-round population-level effects will be higher for species that migrate outside Canada. For instance, in the United States a median estimate of 2.4 billion birds are killed annually by cats (Loss et al. 2013), and a substantial proportion of these birds will have been produced in Canada. In the context of severe population declines already observed for many groups (e.g. long-distance migrants:
BirdLife International 2008; grassland breeders, shorebirds, aerial insectivores: NABCI-Canada 2012), human-related activities create additional population pressures for many of Canada's birds.

The estimated number of potential breeders killed annually by specific sectors or sources differs by several orders of magnitude, ranging from fewer than one thousand for routine marine oil and gas activities, to tens of millions for collisions with vehicles, transmission lines, and houses, and over 140 million for cat kills. Most of these activities are known to effect birds at a local scale, although extrapolation to the national level has highlighted the magnitude and potential significance of several widespread impacts, such as cats and building collisions. For other activities, a national scale perspective may lead to important local-scale mortality being overlooked, e.g., regionally concentrated fisheries bycatch. Our geographical assessment revealed the highest cumulative risk to birds in regions of high human population density and related road networks. Southern Alberta and Ontario also 
stood out as areas with potentially high cumulative effects because of a convergence of several human activities in addition to the top three sources, whereas other high risk locations were generally attributable to single mortality sources.

Although these estimates provide new insight into the relative significance of different industrial and other human-related activities to wild birds in Canada, the precision of our review is limited by the availability of relevant information from Canada. The wide confidence ranges around the converted estimates explicitly indicate the considerable uncertainty in our present knowledge of the magnitude of source-specific mortality, so these should be viewed as preliminary estimates pending further refinement, additional research, and increased monitoring and assessment.

\section{Uncertainties and caveats}

Accurate estimation of the magnitude of bird mortality from industrial and other human-related activities is compromised by the need to estimate large-scale national impacts by extrapolating from small studies, often with limited data. Wherever possible, authors directly accounted for known sources of bias, such as variability in detection and scavenging of bird carcasses (e.g., road vehicles: Bishop and Brogan 2013; building collisions: Machtans et al. 2013; wind power: Zimmerman et al. 2013; transmission line collisions: Rioux et al. 2013). Some explicitly assessed the sensitivity of mortality estimates to key parameters such as the number of unowned cats in Canada (Blancher 2013), or the timing of agricultural or oil and gas activities in relation to breeding seasons (Tews et al. 2013, Van Wilgenburg et al. 2013). Overall, we consider that the estimates presented in this issue are likely to be precise to within an order of magnitude, particularly because actual levels of mortality from each source will likely vary significantly from one year to the next.

Some important sources of estimation bias still remain. For instance, the scale of available data may sometimes be mismatched to the scale of human-related activities. The harvest volume from commercial forestry activities is typically reported provincially and not by area cut, while the density of nesting birds is inferred from extrapolating localscale point-counts to Bird Conservation Regions, which do not align with provincial boundaries (Hobson et al. 2013). Additionally, specific Canadian data for predation rates by cats, pesticide use, and mortality from power generation were also lacking (Blancher 2013; Appendix 1), so the estimates presented here are derived in part using data from other countries or continents. Extrapolations for marine oil and gas were based on untested assumptions, with few data available to inform these estimates (Ellis et al. 2013).

Estimates of effects from most sources could be improved by a better understanding of the seasonal distribution of mortality. For instance, the proportion of industrial activities that occur within the breeding season had to be approximated for several sources (e.g., forestry: Hobson et al. 2013; oil and gas: Van Wilgenburg et al. 2013). Species-composition of the kill is also poorly known for many human activities (e.g., vehicle collisions: Bishop and Brogan 2013; transmission line collisions: Rioux et al. 2013), limiting our ability to evaluate potential population-level impacts. Finally, most analyses presented here were designed to estimate direct annual kill of individual birds or destruction of nests. Estimates for most mortality sources that also involve significant clearing or alteration of habitat do not reflect the total long-term impact of the activity on bird populations because most analyses did not account for additional long-term impacts, e.g., via habitat change (Wells et al.2008) or related one-time mortality events, e.g., destruction of nests during initial construction of transmission lines (Rioux et al. 2013).

The stochastic simulation model addressed some of these biases, so that the distributions of potential adult breeder mortality are more likely to reflect the actual impacts of estimated mortality. The confidence limits around median estimates reflect the remaining uncertainty in the input values; for instance, the magnitude of mortality caused by fisheries bycatch or wind power is known with greater precision than that caused by mining activities or terrestrial oil and gas. These estimates all assume that most mortality estimated here is additive to natural mortality, so density-dependence was not incorporated into these conversions. The stochastic simulation model did make some simplifying assumptions, such as assigning age of first breeding to the second year of life for all but the seabirds, which would overestimate the number of potential breeders when breeding begins later, and by using nest success estimates that assume that nests were destroyed at the beginning of nesting, which would underestimate the number of potential breeders if nest destruction occurred later in the season. An important potential bias of the modeling process was the use of representative vital rates from only a few species, except the landbirds. In the future, more detailed estimates of species-specific kills could be incorporated with models using their species-specific vital rates to properly assess the effects of any particular mortality source. Finally, there are some considerations that the conversion to potential adult breeders could not incorporate. Long-lived, lowfecundity species take longer to recover from population perturbations, and mortality for these species is more likely to be additive than for shorter lived high-fecundity species. Additionally, long-lived, low-fecundity species tend to have much smaller population sizes, so a greater portion of the population is removed with each potential adult killed.

The risk mapping also relied on some important assumptions, specifically that mortality from each source was spread across the landscape in proportion to its existing spatial intensity. This is certainly not the case; forestry companies do not harvest equally across their tenure area and not every communication 
tower or wind turbine kills the average number of birds. However, adopting this assumption was necessary to create a first order spatial representation of the distribution of avian mortality risk across Canada.

The values considered here represent the current best estimates of source-specific annual bird mortality for Canada across all species groups and age classes, although a few sectoral mortality estimates must be considered to be quite preliminary, and there is some inherent uncertainty in all estimates. Moreover, because the magnitude of the estimates is likely to be fairly accurate, with true mortality levels contained within the estimation range, the relative ranking of mortality sources is unlikely to change substantially with improved precision.

\section{CONCLUSIONS AND IMPLICATIONS}

From a conservation perspective, it is now important to develop a more complete understanding of the population level effects of human-related avian mortality within and across sectors, at relevant spatial scales. Sources such as window strikes at houses cause high levels of mortality nation-wide, but this mortality is not spread equally across different species or families. Longcore et al. (2013) found similarly variable population impacts of communication tower collisions. Marine fisheries bycatch was not among the highest-ranking sources of mortality nation-wide, yet it kills disproportionately high numbers of birds from particular regional populations. Our assessment did not consider the fact that certain populations or species may still manifest a population-level consequence through additive effects of several mortality sources, even though each source individually would not be expected to show such an effect. Understanding these cumulative effects will not be possible until species-specific kill rates are available for all sectors. In the interim, those habitats or areas of the country where many sectors operate together are places where these multiple stressors have the potential to combine and create such a cumulative impact.

This synthesis and accompanying papers focus primarily on direct mortality of birds and destruction of nests resulting from human activities, but do not consider the potential longer term effects on birds from habitat changes. Wind turbines, for example, cause mortality by nest-destruction during construction as well as through collision mortality during operation. Indeed, recent evidence suggests that initial construction may sometimes pose a greater overall threat to birds (Pearce-Higgins et al. 2012). Commercial forestry, terrestrial oil and gas, and mining are further examples of activities where there may be significant longer term or broader scale effects of habitat modification that are not addressed here. Furthermore, mortality rates may change in the future for industries undergoing rapid rates of development, such as wind facilities, which are predicted to expand ten-fold in Canada over the next 10-15 years (CanWEA 2013). Human activities currently contributing relatively little to total mortality may therefore present a greater risk in years to come.

The complex relationships among all ecological factors regulating avian populations, and particularly migratory birds, require consideration of factors operating at points throughout the entire life cycle (Faaborg et al. 2010). For example, if wintering habitat conditions are not limiting, human-related mortality may be additive. However, if wintering habitat becomes limiting, human-related mortality may shift to being compensatory and its influence on population regulation may change. Improved understanding of species composition of mortality events, the magnitude of mortality of migrants south of Canada, and survival estimates at each life stage will be required to effectively model the demography of affected populations, particularly if bird conservation objectives include maintaining source-specific mortality from humanrelated causes below certain levels (e.g., McGowan and Ryan 2009, Runge et al. 2009, Dillingham and Fletcher 2011).

Insight into the relative magnitude of different human-related sources of mortality provides a valuable tool for guiding management, and affords additional perspectives for prioritizing conservation and research initiatives for Canada's birds. We propose four key areas for future research or management. First, to enable more precise analyses and impact modeling, we recommend additional Canadian research into the magnitude of bird effects for data-poor sectors, e.g., pesticides, and the species likely affected, and into particular aspects of mortality, e.g., species composition and seasonal timing of the kill. Second, our results highlight the value of increased efforts to minimize impacts of widespread and generalized low-intensity human-related activities that create nationally high levels of mortality but could be mitigated at local scales, e.g., cats and buildings. Such investments could include local approaches using outreach and other available conservation tools. Third, we recommend specifically targeting those mortality sources identified as having population-level effects at regional or national levels for priority conservation action. Finally, we encourage further assessments that integrate the effects on populations across multiple sectors to truly understand the impacts of all mortality sources on priority species. Such mitigation efforts can reduce human-related impacts on birds if appropriately directed (as shown by e.g., Nocera et al. 2005, 2007: changing the timing of agricultural activities to reduce impacts on grassland breeders; Gehring et al. 2009: changing lights on communication towers to reduce collision mortality; and Løkkeborg 2011: modifying fishing gear to reduce bycatch of seabirds.

Given that the relative ranking of mortality sources considered here is unlikely to change substantially even with increased precision, an immediate focus should consider mitigation of those mortality sources with the highest magnitudes at the 
national level, e.g., cats and collisions. At the same time, scientists should try to identify and better understand potential population-level impacts on populations or species, at appropriate geographical scales. Effective application of these findings to the conservation of Canadian birds will require constructive collaboration among the public and various levels of government, nongovernmental organizations, and industries within Canada. This assessment should help target these initiatives appropriately to improve the population and conservation status of birds within Canada, as well as the continental conservation status for migratory species.

Responses to this article can be read online at: http://www.ace-eco.org/issues/responses.php/581

\section{Acknowledgments:}

This research was instigated and funded by Environment Canada. Thank you to Amos Chow for assistance in compiling some of the mapping data, to Beau MacDonald for providing quality-controlled communication tower data, to Alyssa Serena for compiling provincial game-bird harvest numbers, to Steve Van Wilgenburg and Keith Hobson for providing their landbird demographic rates, and to Peter Blancher for unpublished family-level abundance estimates. Finally, we thank the authors of all the papers and reports cited here on individual sources of human-related avian mortality for their feedback and clarification of their mortality estimates.

\section{LITERATURE CITED}

Anderson, D. R., and K. P. Burnham. 1976. Population ecology of the mallard IV: the effect of exploitation on survival. Publication 128. U.S. Department of the Interior, U.S. Geological Survey, Reston, Virginia, USA.

Arnold, T. W., and R. M. Zink. 2011. Collision mortality has no discernible effect on population trends of North American birds. PLoS ONE 6(9):e24708. http://dx.doi.org/10.1371/ journal.pone.0024708

Balogh, A. L., T. B. Ryder, and P. P. Marra. 2011. Population demography of Gray Catbirds in the suburban matrix: sources, sinks and domestic cats. Journal of Ornithology 152:717-726. http://dx.doi.org/10.1007/s10336-011-0648-7

Bayne, E. M., C. A. Scobie, and M. Rawson-Clark. 2012. Factors influencing the annual risk of bird-window collisions at residential structures in Alberta, Canada. Wildlife Research 39(7):583-592.

BirdLife International. 2008. State of the world's birds: indicators for our changing world. BirdLife International, Cambridge, UK.
Bishop, C. A., and J. M. Brogan. 2013. Estimates of avian mortality attributed to vehicle collisions in Canada. Avian Conservation and Ecology 8(2): 2. http://dx.doi.org/10.5751/ ACE-00604-080202

Blancher, P. J. 2002. Importance of breeding birds exported from Canada - information on landbirds and shorebirds. Unpublished report to the North American Bird Conservation Initiative Canada National Council. Ottawa, Ontario, Canada.

Blancher, P. J. 2013. Estimated number of birds killed by house cats (Felis catus) in Canada. Avian Conservation and Ecology 8(2): 3. http://dx.doi.org/10.5751/ACE-00557-080203

Canadian Wind Energy Association (CanWEA). 2013. Powering Canada' future. CanWEA, Ottawa, Ontario, Canada. [online] URL: http://www.canwea.ca/pdf/Canada\% 20Current\%20Installed\%20Capacity e.pdf

Committee on the Status of Endangered Wildlife in Canada (COSEWIC). 2010. COSEWIC assessment and status report on the Bobolink Dolichonyx oryzivorus in Canada. COSEWIC, Ottawa, Ontario, Canada. [online] URL: http:// registrelep-sararegistry.gc.ca/default.asp?lang=En\&n=7019552A-1

Crooks, K. R., and M. E. Soulé. 1999. Mesopredator release and avifaunal extinctions in a fragmented system. Nature 400:563-566. http://dx.doi.org/10.1038/23028

Dillingham, P. W., and D. Fletcher. 2011. Potential biological removal of albatrosses and petrels with minimal demographic information. Biological Conservation 144:1885-1894. http:// dx.doi.org/10.1016/j.biocon.2011.04.014

Ellarson, R. S. 1956. A study of the Old-squaw Duck on Lake Michigan. Dissertation. University of Wisconsin, Madison, Wisconsin, USA.

Ellis, J. I., S. I. Wilhelm, A. Hedd, G. S. Fraser, G. J. Robertson, J.-F. Rail, M. Fowler, and K. H. Morgan. 2013. Mortality of migratory birds from marine commercial fisheries and offshore oil and gas production in Canada. Avian Conservation and Ecology 8(2): 4. http://dx.doi.org/10.5751/ACE-00589-080204

Faaborg, J., R. T. Holmes, A. D. Anders, K. L. Bildstein, K. M. Dugger, S. A. Gauthreaux Jr., P. Heglund, K. A. Hobson, A. E. Jahn, D. H. Johnson, S. C. Latta, D. J. Levey, P. P. Marra, C. L. Merkord, E. Nol, S. I. Rothstein, T. W. Sherry, T. S. Sillett, F. R. Thomson III, and N. Warnock. 2010. Recent advances in understanding migration systems of New World land birds. Ecological Monographs 80:3-48. http://dx.doi. org/10.1890/09-0395.1

Gehring, J., P. Kerlinger, and A. M. Manville II. 2009. Communication towers, lights, and birds: successful methods of reducing the frequency of avian collisions. Ecological Applications 19:505-514. http://dx.doi.org/10.1890/07-1708.1 
Hobson, K. A., A. G. Wilson, S. L. Van Wilgenburg, and E. M. Bayne. 2013. An estimate of nest loss in Canada due to industrial forestry operations. Avian Conservation and Ecology 8(2): 5. http://dx.doi.org/10.5751/ACE-00583-080205

Jenks, G. F. 1967. The data model concept in statistical mapping. International Yearbook of Cartography 7:186-190.

Jensen, J. R. 2005. Introductory digital image processing: a remote sensing perspective. Third edition. Pearson Prentice Hall, Upper Saddle River, New Jersey, USA.

Johnston, J. P., W. J. Peach, R. D. Gregory, and S. A. White. 1997. Survival rates of tropical and temperate passerines: a Trinidadian perspective. American Naturalist 150:771-789. http://dx.doi.org/10.1086/286093

Klem, D., Jr. 2009. Preventing bird-window collisions. Wilson Journal of Ornithology 121:314-321. http://dx.doi. org/10.1676/08-118.1

Løkkeborg, S. 2011. Best practices to mitigate seabird bycatch in longline, trawl and gillnet fisheries-efficiency and practical applicability. Marine Ecology Progress Series 435:285-303. http://dx.doi.org/10.3354/meps09227

Longcore, T., C. Rich, P. Mineau, B. MacDonald, D. G. Bert, L. M. Sullivan, E. Mutrie, S. A. Gauthreaux Jr., M. L. Avery, R. L. Crawford, A. M. Manville II, E. R. Travis, and D. Drake. 2012. An estimate of avian mortality at communication towers in the United States and Canada. PLoS ONE 7(4): e34025. http://dx.doi.org/10.1371/journal.pone.0034025

Longcore, T., C. Rich, P. Mineau, B. MacDonald, D. G. Bert, L. M. Sullivan, E. Mutrie, S. A. Gauthreaux Jr., M. L. Avery, R. L. Crawford, A. M. Manville II, E. R. Travis, and D. Drake. 2013. Avian mortality at communication towers in the United States and Canada: which species, how many, and where? Biological Conservation 158:410-419. http://dx.doi.org/10.1016/ j.biocon.2012.09.019

Loss, S. R., T. Will, and P. P. Marra. 2012. Direct humancaused mortality of birds: improving quantification of magnitude and assessment of population impact. Frontiers in Ecology and the Environment 10:357-364. http://dx.doi. org/10.1890/110251

Loss, S. R., T. Will, and P. P. Marra. 2013. The impact of freeranging domestic cats on wildlife of the United States. Nature Communications 4:1396. http://dx.doi.org/10.1038/ncomms2380

Machtans, C. S., C. H. R. Wedeles, and E. M. Bayne. 2013. A first estimate for Canada of the number of birds killed by colliding with building windows. Avian Conservation and Ecology 8(2): 6. http://dx.doi.org/10.5751/ACE-00568-080206

Manville, A. M., II. 2009. Towers, turbines, power lines, and buildings - steps being taken by the U.S. Fish and Wildlife
Service to avoid or minimize take of migratory birds at these structures. Pages 262-272 in T. D. Rich, C. Arizmendi, D. W. Demarest, and C. Thompson, editors. Tundra to tropics: connecting birds, habitats, and people. Proceedings of the 4th International Partners in Flight Conference, Partners in Flight, McAllen, Texas, USA.

McGowan, C. P., and M. R. Ryan. 2009. A quantitative framework to evaluate incidental take and endangered species population viability. Biological Conservation 142:3128-3136. http://dx.doi.org/10.1016/j.biocon.2009.08.012

Miller, M. R., and D. C. Duncan. 1999. The northern pintail in North America: status and conservation needs of a struggling population. Wildlife Society Bulletin 27:788-800.

Mineau, P., and M. Whiteside. 2013. Pesticide acute toxicity is a better correlate of U.S. grassland bird declines than agricultural intensification. PLoS ONE 8(2):e57457. http://dx. doi.org/10.1371/journal.pone.0057457

Nichols, J. D., M. C. Runge, F. A. Johnson, and B. K. Williams. 2007. Adaptive harvest management of North American waterfowl populations: a brief history and future prospects. Journal of Ornithology 148(Suppl. 2):S343-S349. http://dx. doi.org/10.1007/s10336-007-0256-8

Nocera, J. J., G. Forbes, and G. R. Milton. 2007. Habitat relationships of three grassland breeding bird species: broadscale comparisons and hayfield management implications. Avian Conservation and Ecology 2(1): 7. [online] URL: http:// www.ace-eco.org/vol2/iss1/art7/

Nocera, J. J., G. J. Parsons, G. R. Milton, and A. H. Fredeen. 2005. Compatibility of delayed cutting regime with bird breeding and hay nutritional quality. Agriculture, Ecosystems \& Environment 107:245-253. http://dx.doi.org/10.1016/j. agee.2004.11.001

North American Bird Conservation Initiative Canada (NABCI-Canada). 2012. The state of Canada's birds, 2012. Environment Canada, Gatineau, Quebec, Canada.

Pearce-Higgins, J. W., L. Stephen, A. Douse, and R. H. W. Langston. 2012. Greater impacts of wind farms on bird populations during construction than subsequent operation: results of a multi-site and multi-species analysis. Journal of Applied Ecology 49:386-394. http://dx.doi.org/10.1111/ j.1365-2664.2012.02110.x

Piatt, J. F., D. N. Nettleship, and W. T. Threlfall. 1984. Net mortality of Common Murres Uria aalge and Atlantic Puffins Fratercula acrtica in Newfoundland, 1951-1981. Pages 196-206 in D. N. Nettleship, G. Sanger, and P. F. Springer, editors. Marine birds: their feeding ecology and commercial fisheries relationships. Special publication. Canadian Wildlife Service, Ottawa, Ontario, Canada. 
Price, I. M., and J. G. Nickum. 1995. Aquaculture and birds: the context for controversy. Colonial Waterbirds 18 (Special Publication):33-45. http://dx.doi.org/10.2307/1521522

Prairie Habitat Joint Venture. 2008. Prairie Habitat Joint Venture Implementation Plan 2007 - 2012. Report of the Prairie Habitat Joint Venture. Environment Canada, Edmonton, Alberta, Canada.

Rioux, S., J.-P. L. Savard, and A. A. Gerick. 2013. Avian mortalities due to transmission line collisions: a review of current estimates and field methods with an emphasis on applications to the Canadian electric network. Avian Conservation and Ecology 8(2): 7. http://dx.doi.org/10.5751/ ACE-00614-080207

Runge, M. C., J. R. Sauer, M. L. Avery, B. F. Blackwell, and M. D. Koneff. 2009. Assessing allowable take of migratory birds. Journal of Wildlife Management 73:556-565. http://dx. doi.org/10.2193/2008-090

Tews, J., D. G. Bert, and P. Mineau. 2013. Estimated mortality of selected migratory bird species from mowing and other mechanical operations in Canadian agriculture. Avian Conservation and Ecology 8(2): 8. http://dx.doi.org/10.5751/ ACE-00559-080208

Thomas, P. J., P. Mineau, R. F. Shore, L. Champoux, P. A. Martin, L. K. Wilson, G. Fitzgerald, and J. E. Elliott. 2011. Second generation anticoagulant rodenticides in predatory birds: probabilities characterisation of toxic liver concentrations and implications for predatory bird populations in Canada. Environment International 37:914-920. http://dx. doi.org/10.1016/j.envint.2011.03.010

Timoney, K. P, and R. A. Ronconi. 2010. Annual bird mortality in the bitumen tailings ponds in northeastern Alberta, Canada. Wilson Journal of Ornithology 122:569-576. http:// dx.doi.org/10.1676/09-181.1

Van Wilgenburg, S. L., K. A. Hobson, E. M. Bayne, and N. Koper. 2013. Estimated avian nest loss associated with oil and gas exploration and extraction in the Western Canadian Sedimentary Basin. Avian Conservation and Ecology 8(2): 9. http://dx.doi.org/10.5751/ACE-00585-080209

Wells, J., S. Casey-Lefkowitz, G. Chavarria, and S. Dyer. 2008. Danger in the nursery: impacts on birds of tar sands oil development in Canada's boreal forest. Natural Resources Defense Council, New York, NY, USA.

Wiese, F. K., and G. J. Robertson. 2004. Assessing seabird mortality from chronic oil discharges at sea. Journal of Wildlife Management 68:627-638. http://dx.doi.org/10.2193/0022-541X (2004)068[0627:ASMFCO]2.0.CO;2

Williams, B. K., and F. A. Johnson. 1995. Adaptive management and the regulation of waterfowl harvests. Wildlife Society Bulletin 23:430-436.
Zimmerling, J. R., A. Pomeroy, M. d'Entremont, and C. M. Francis. 2013. Canadian estimate of bird mortality due to collisions and direct habitat loss associated with wind turbine developments. Avian Conservation and Ecology 8(2): 10. http://dx.doi.org/10.5751/ACE-00609-080210

Zydelis, R., D. Esler, M. Kirk, and W. S. Boyd. 2009. Effects of off-bottom shellfish aquaculture on winter habitat use by molluscivorous sea ducks. Aquatic Conservation: Marine and Freshwater Ecosystems 19:34-42. http://dx.doi.org/10.1002/ aqc. 977

Zydelis, R., D. Esler, W. S. Boyd, D. L. Lacroix, and M. Kirk. 2006. Habitat use by wintering Surf and White-winged Scoters: effects of environmental attributes and shellfish aquaculture. Journal of Wildlife Management 70:1754-1762. http://dx.doi.org/10.2193/0022-541X(2006)70[1754:HUBWSA] 2.0.CO;2 


\section{Appendix 1: Unpublished reports on sources of mortality cited in the text.}

The following reports are not peer reviewed and should not be cited as such. The authors of the main paper for which these materials form a supplementary appendix make no expression on the validity of individual portions of or computations in the papers aside from what was extracted for use with noted caveats in the main paper. 


\title{
Avian Incidental Take due to Roadside Maintenance Operations in Canada
}

\author{
Prepared by \\ ESSA Technologies Ltd. \\ and \\ Arbor Vitae Environmental Services Ltd. \\ for \\ Environment Canada
}

June 2010 
Suggested Citation: Abraham, D., D. Pickard and C. Wedeles. 2010. Avian Incidental Take due to Roadside Maintenance Operations in Canada. Report Prepared by ESSA Technologies Ltd. and Arbor Vitae Environmental Services Ltd. for Environment Canada. 31 pp. + appendices 


\section{Table of Contents}

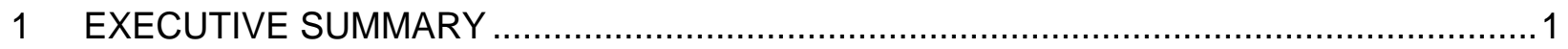

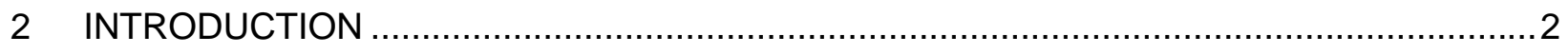

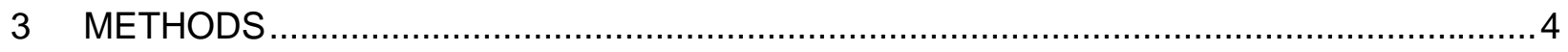

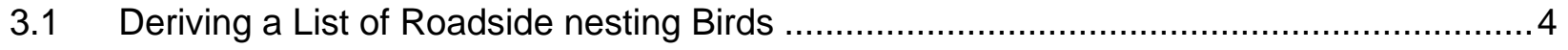

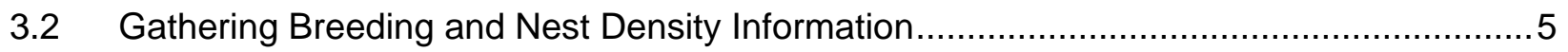

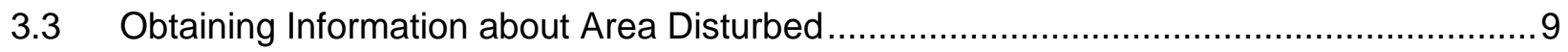

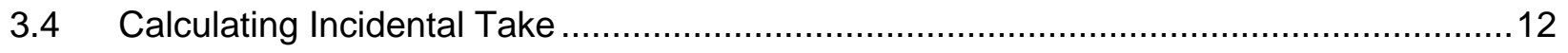

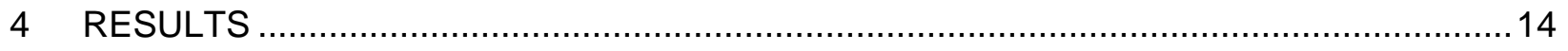

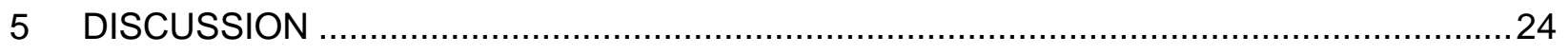

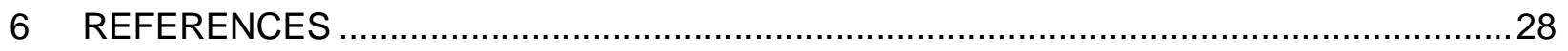

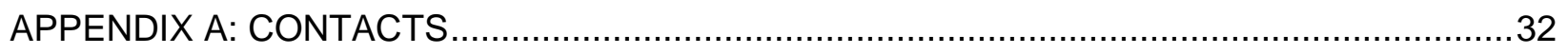

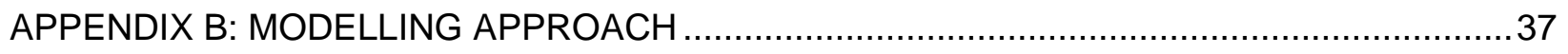

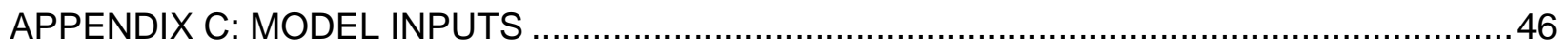




\section{List of Tables}

Table 1: List of roadside nesting bird species. 4

Table 2: Model parameter input information for birds. 5

Table 3. Combinations of habitat type and quality for each focal species. 8

Table 4. Road classes included in the GIS analysis ................................................... 10

Table 5. General habitat types used in the analysis. ........................................................ 10

Table 6. The extent of roads in Canada, by province and territory.................................... 11

Table 7. Number of kilometers of road within the breeding range for 6 focal species. .............. 12

Table 8. Incidental take for 6 focal species by province/territory. . .................................... 14

Table 9. Incident take for 6 focal species for all of Canada. .......................................... 16

Table 10. Incidental take for 12 non-focal species by province/territory............................17

Table 11. Total annual estimated incidental take for 18 bird species affected by roadside

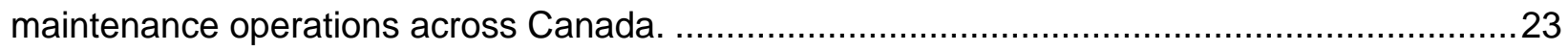

Table 12. Total annual estimated take for 6 focal species and 12 non-focal species...............23

Table 13: Example of interim results of the Discount.fcn() for killdeer in Ontario....................42

Table 14: Example of model output for killdeer............................................................ 44 


\section{EXECUTIVE SUMMARY}

ArborVitae Environmental Services Ltd. and ESSA Technologies Ltd. generated preliminary estimates of the magnitude of avian incidental take due to roadside maintenance operations across Canada. Eighteen roadside nesting species, all protected under the Migratory Birds Convention Act, were identified through the literature and expert advice. To model the impacts on these species, this study took a focal-species approach, in which estimates of incidental take were modeled for 6 focal species and then extrapolated to 12 other species which had similar ecologies. The estimates of take for the focal species were based on:

- $\quad$ their nesting ecology (i.e. nesting dates, number of eggs laid, incubation periods) and range in Canada;

- $\quad$ preferred nesting habitats relative to the availability of the habitats along roadsides; and

- the amount of road and maintenance activities conducted in each province.

We used a modeling approach which integrated the information above with assumed and calculated distributions of nesting period, road maintenance schedules, egg laying, etc.

Estimates of take were made for these species using a combination of modeling and extrapolation. Incidental take ranged from 7 (Lark Sparrow) to 820,000 (American Robin) individuals per year across Canada. We estimate that approximately 861,000 nestlings, eggs, and adults (only waterfowl adults are susceptible to incidental take) are killed by incidental take per year. However, this analysis, although very detailed, is subject to a number of caveats which suggest that the results should be interpreted with considerable caution.

There are no published criteria for what constitutes biologically significant levels of incidental take for bird populations. However, a widely accepted criterion for identifying key habitat sites for population conservation may serve as a suitable surrogate. Sites believed to support at least $1 \%$ of a Canadian population are considered to be key habitat sites, and their loss would potentially have a significant detrimental impact on the total population. By extension, losses to incidental take of $1 \%$ or more of a species Canadian population could be considered biologically significant (C. Machtans, pers. com.).

As a proportion of total Canadian populations, take was estimated to be less than $1 \%$ for all species, ranging from $0.0057 \%$ (Clay-colored Sparrow) to $0.5880 \%$ (American Robin). According to the $1 \%$ criterion, incidental take due to roadside maintenance operations is not a biologically significant mortality factor in Canada. 


\section{INTRODUCTION}

The inadvertent destruction of birds and/or their nests and young occurs in Canada during otherwise legitimate operations in a variety of sectors, including forestry, mining, agriculture, electrical generation and transmission, fishing, structures, roadside maintenance and road construction. Such "incidental take" is an important factor in bird conservation and management, and Environment Canada has identified a need to better understand the magnitude and significance of the issue.

The objective of this project was to generate defensible species specific estimates of the number of birds killed annually due to roadside maintenance activities in Canada, by province/territory, e.g., for every hectare of roadside affected by mowing and/or brushing, an average of $X$ number of individuals of species $Y$ are killed each year. Only bird species that breed in Canada and are covered under the Migratory Birds Convention Act (MBCA) were included; species not protected by the MBCA include raptors, corvids, blackbirds, gallinaceous birds, and some others not explicitly mentioned in the Act. The temporal scope of the project was the breeding season, so winter maintenance activities such as snow management were excluded. Roadside maintenance activities in Canada include mowing, brushing (shrub cutting), and tree trimming. Some jurisdictions also use herbicides (e.g., Newfoundland/Labrador) to control vegetation, but this type of impact falls under the category of substances harmful to birds (as regulated by S5.1 of the MBCA), and was beyond the scope of the project. All roads for which roadside vegetation is managed by mechanical means were included in the analysis. Long-term resource roads that provide access to the back country were included, but short-term resource roads were out of scope.

This report documents a research and modeling effort to estimate the magnitude of avian mortality due to roadside maintenance activities, such as mowing and shrub brushing, across Canada. Roadside vegetation is managed for a variety of reasons, including safety (Forman et al. 2003; Jacobson 2005), aesthetics (Jacobson 2005), the control of invasive plant species, and in preparation for snow removal. Mowing-related avian mortality in roadside habitats is understood by many researchers to occur (e.g., Forman et al. 2003; Maguire 2007), but few studies have attempted to quantify it, and none have attempted to quantify it on a national scale. Even comprehensive reviews on the short- and long-term ecological effects of roads do not cover mortality from mowing equipment (Spellerberg 1998; Trombulak and Frissell 2000; Forman et al. 2003). In agricultural areas, bird use of strip-cover habitats such as road rights-ofway, fencerows, farmstead shelterbelts and grassed waterways can be high (Best et al. 1995). Such habitats provide nest sites, particularly shrubs and trees that are usually not available in the surrounding landscape. For bird species associated with roadside habitats, incidental take by mowing and other right-of-way maintenance operations may be a significant source of mortality.

A thorough literature review, combined with information from experts in the growing field of road ecology (see Appendix A), showed that data related to avian mortality due to roadside maintenance activities are virtually nonexistent. In North Dakota, Cook and Daggett (1995) reported "major losses" of birds to road right-of-way mowing; this loss includes ducks (34\% of roadside duck nests have not hatched by the time the mowing occurs) and fledgling birds that could not escape the mowers. The same study estimated that 4,500 ducks are killed annually in the Prairie Pothole Region of North Dakota, but it isn't clear if this mortality is mowing-related or due to vehicle strikes. In a study in lowa, roadside mowing destroyed only 2 of 98 nests in 34 roadside plots that covered 10.2 hectares (Camp and Best 1994); predation was the major 
mortality factor. In Illinois, 13 of 91 ring-necked pheasant roadside nests were destroyed by mowing (Joselyn et al. 1968). A California study reported the inadvertent destruction of 4 burrowing owl nests on an $800 \mathrm{~m}$ stretch of road due to roadside maintenance activities; 3 adult owls were also killed (Catlin and Rosenberg 2006). In a Danish study, roadside mowing was confirmed responsible for the loss of 1 of 3 skylark nests over a $4.7 \mathrm{~km}$ stretch of roadway (Laursen 1981). In Oregon, two maintenance workers estimated they killed between 400 and 600 birds during early July mowing operations (Braun et al. 1978, in Dale 1993). Jackson and Jackson (2000) characterized some gravel roadsides as ecological sinks for killdeer due to the negative impacts of pesticides and "destruction of eggs and chicks"; it isn't clear, however, if this destruction was due to roadside maintenance or to some other form of road-related activity. In other published studies, mortality due to roadside mowing is implied by results that show lower productivity following mowing (Dale et al. 1997), higher nesting success where mowing was delayed (e.g., Oetting and Cassel 1971; Dale 1993; Leif 2004), or higher nest densities in unmowed vs. mowed roadside (Berner 1984:32 in MDNR 2005). In many instances, maintenance-related mortality is understood to occur, but no empirical evidence is provided (e.g., Farris et al. 1977; Camp and Best 1994; Jacobson 2005; Belanger et al. 2006; Maguire 2007). 


\section{METHODS}

Given the nearly total absence of empirical data about bird mortality as a result of roadside maintenance operations, we chose a modelling approach to estimate incidental take (Appendix B). To do this, we needed to: 1) derive a list of bird species likely to nest in roadside habitats; 2) gather breeding and nest density information for those species; and 3) obtain information about the area disturbed by maintenance activities in each province and territory.

\subsection{Deriving a LiSt of Roadside nesting BiRdS}

A search of the literature established that no recognized "roadside nesting guild" of birds exists in published works, including Forman et al. (2003). We used a combination of expert opinion and literature to build a list of species likely to nest in road rights-of-way across Canada. As noted earlier, only species covered by the MBCA were considered for inclusion, which explains the otherwise noteworthy absence from the list of species like red-winged blackbird and ringnecked pheasant. The species on the list were then grouped according to general habitat requirements based on the literature listed in Table 1. For each group, a single focal species was selected to represent the group's risk of exposure to mortality from mowing and brushing operations. This approach was adapted from the approach taken by Tews et al. (2009) for the incidental take analysis of the agriculture sector.

The list of roadside nesting birds (Table 1), with habitat groupings and focal species, was then reviewed by grassland bird experts from Environment Canada (K. Lindsay, B.Dale) and revised accordingly.

Table 1: List of roadside nesting bird species, organized into groups according to general habitat characteristics. Focal species for each group are indicted in bold text.

\begin{tabular}{|c|c|c|c|}
\hline General Habitat Characteristics & Nest Elevation & Species & Source \\
\hline $\begin{array}{l}\text { bare ground or sparse, low } \\
\text { vegetation }\end{array}$ & ground & Killdeer & $\begin{array}{l}\text { Oetting and Cassel 1971; Peck and } \\
\text { James 1983; Best et al. } 1995\end{array}$ \\
\hline denser, taller vegetation & elevated & American Goldfinch & $\begin{array}{l}\text { Peck and James 1987; McGraw and } \\
\text { Middleton } 2009\end{array}$ \\
\hline denser, taller vegetation & ground & Eastern Meadowlark & $\begin{array}{l}\text { Hergenrader 1962; Peck and James } \\
\text { 1987; Warner 1992; Best et al. 1995; } \\
\text { Leif 2004; MDNR 2005; Shochat et al. } \\
2005\end{array}$ \\
\hline denser, taller vegetation & ground & Savannah Sparrow & $\begin{array}{l}\text { Peck and James 1987; Best et al. } \\
\text { 1995; MDNR 2005; Wheelwright and } \\
\text { Rising 2008; Brenda Dale, pers. com. }\end{array}$ \\
\hline denser, taller vegetation & ground & Western Meadowlark & $\begin{array}{l}\text { Hergenrader 1962; Warner 1992; } \\
\text { Camp and Best 1994; Best et al. 1995; } \\
\text { Leif 2004; MDNR 2005 }\end{array}$ \\
\hline open shrub/tree; woodland edges & ground and elevated & Mourning Dove & $\begin{array}{l}\text { Hergenrader 1962; Oetting and Cassel } \\
\text { 1971; Leif } 2004\end{array}$ \\
\hline open shrub-tree; woodland edges & ground and elevated & Song Sparrow & $\begin{array}{l}\text { Peck and James 1987; Warner 1992; } \\
\text { Camp and Best 1994; Best et al. } 1995\end{array}$ \\
\hline open shrub/tree; woodland edges & elevated & Indigo Bunting & Peck and James 1987; Payne 2006 \\
\hline grass-shrub mix & ground and elevated & Clay-colored Sparrow & Brenda Dale, pers. com. \\
\hline grass-shrub mix & elevated & American Robin & Best et al. 1995 \\
\hline
\end{tabular}




\begin{tabular}{|c|c|c|c|}
\hline grass-shrub mix & $\begin{array}{l}\text { ground } \\
\text { ground }\end{array}$ & $\begin{array}{l}\text { Vesper Sparrow } \\
\text { Lark Sparrow }\end{array}$ & $\begin{array}{l}\text { Peck and James 1987; Warner 1992; } \\
\text { Camp and Best 1994; Best et al. 1995; } \\
\text { Brenda Dale, pers. com. } \\
\text { Peck and James } 1987\end{array}$ \\
\hline grassy fields; meadows & ground & American Black Duck & Peck and James 1983 \\
\hline grassy fields; meadows & ground & Blue-winged Teal & Peck and James 1983 \\
\hline grassy fields; meadows & ground & Gadwall & Peck and James 1983 \\
\hline grassy fields; meadows & ground & Mallard & Peck and James 1983 \\
\hline grassy fields; meadows & ground & Northern Pintail & Peck and James 1983 \\
\hline grassy fields; meadows & ground & Northern Shoveler & Peck and James 1983 \\
\hline
\end{tabular}

\subsection{Gathering Breeding and Nest Density Information}

In order to model the number of individual birds (adults, eggs and nestlings) that are killed annually by roadside mowing and brushing operations, we needed to assemble breeding and nest density information for each of the focal species on the bird list (Table 2).

Table 2: Model parameter input information for birds.

\begin{tabular}{ll}
\hline Parameter & Description \\
\hline egg dates & the earliest and latest dates on which viable eggs have \\
& been recorded in nests (Peck and James 1987:2) \\
incubation time & average \# days from date of last egg laid to hatch \\
nestling time & average \# days in nest after hatching \\
temporal distribution of egg-laying & use start date regression model to determine proportion \\
& of nests initiated by date (as per Tews et al. 2009) \\
temporal distribution of fledging & assumed to be the same as distribution of egg-laying, \\
& i.e., fixed number of days for incubation and nestling \\
& time \\
clutch size & average number of eggs per nest \\
nest densities & average \# nests per hectare, in each of 4 general \\
& habitat types \\
\hline
\end{tabular}

The assumptions inherent in the parameter definitions in Table 2 are:

- 1 brood per pair and no re-nesting (as per Tews et al. 2009); most of the focal species on the list of roadside birds are known or suspected to be capable of raising second broods (killdeer, savannah sparrow, song sparrow, vesper sparrow) (Peck and James 1987), but further research is needed to gather the information necessary to incorporate this factor into the calculations of take

- $100 \%$ of eggs laid are fertile and represent a bird for the purposes of incidental take

- hatching success is $100 \%$, i.e., the number of fledglings equals the number of eggs

- the nestlings of altricial species stay in or near the nest until fledged, and are therefore vulnerable to mowing/brushing equipment for the entire nestling period; the nestlings of killdeer (the only precocial species on the list) are led away from the nest by their parents within 2 days of hatching (Davis 1943 in Ankney 1985), travelling long distances (greater than $100 \mathrm{~m}$ ) to brood rearing habitats (Powell 1993), at which time they are no longer vulnerable to mowing/brushing equipment 
Natural mortality was not deducted from our estimates (pers. comm. C. Machtans and B. Dale, Environment Canada). The consensus was that a nestling killed by a mower constitutes incidental take no matter if that nestling would have later died of other anthropogenic or nonanthropogenic causes. These assumptions will therefore generate a maximum estimate of incidental take.

To maximize efficiency, we used the breeding information compiled by Tews et al. (2009) ${ }^{1}$ whenever possible, i.e., for focal species that were common to both projects. For other focal species, we used Peck and James $(1983,1987)$ and the Birds of North America online as resources.

To determine estimates for nest densities by habitat type, we used the Canadian Breeding Bird Census (CBBC) database (see Kennedy et al. 1999) which reports adult densities (birds/km²) derived from "spot- or territory-mapping" censuses from 640 sites spread across 76 of Canada's 194 ecoregions (Figure 1) (Blancher 2002).

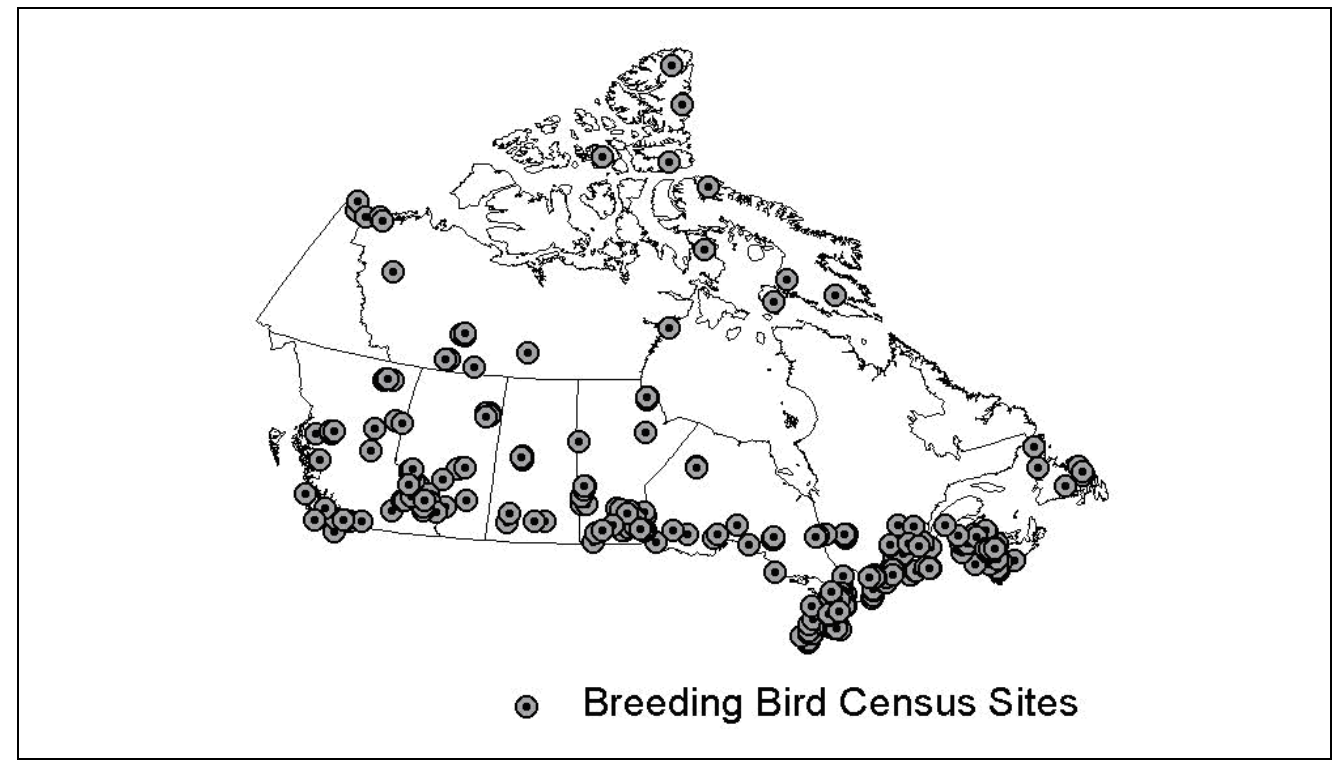

Figure 1. Canadian Breeding Bird Census sites (from Blancher 2002).

For each of our focal species, we mapped the habitats in the CBBC to 4 general habitat types Forest, Shrubland, Grassland And Wetland. For example, "grazed pasture" and "clover-alfalfatimothy field" both mapped to Grassland, whereas "old field with hedgerows" and "tall bottomland willow stand" both mapped to Shrubland. This both simplified and standardized the relevant habitats, and made them compatible with the Land Cover classes available to us for the GIS analysis (see Table 5, below). For provinces/territories within a species range that had no density estimates in the $\mathrm{CBBC}$, we used densities from the nearest neighbouring province/territory for which there were data. Where multiple values were available, we used the mean value. The strength of the CBBC is that it provides habitat-specific density estimates by species and by province/territory; its weaknesses are: 1) older data; 2) low sample sizes, precluding fine resolution analysis; and 3) non-random sampling sites, which make it unclear to what extent densities are representative of areas not sampled (Blancher 2002). Additionally,

\footnotetext{
${ }^{1}$ We are indebted to Pierre Mineau, Environment Canada (Ottawa) for access to these data.
} 
the CBBC under-represents the boreal and taiga regions of Canada (see Figure 1, above), and there are no census data for the province of Prince Edward Island.

The bird breeding and density inputs used in the simulation model are shown in Appendix C. Further research and analysis could be done to refine these density estimates (e.g., using the approach taken by Blancher (2002)), including incorporation of data from the Northwest Territories / Nunavut Bird Checklist Survey, and data for Prince Edward Island. Density values can easily be updated in the model if/when better information becomes available.

\section{Accounting for Habitat Quality}

Bird species that occur in roadside habitats tend to be similar to those of the adjacent habitat (Meunier et al. 1999; Belanger et al. 2006). However, road rights-of-way are probably not preferred habitat for any of the species that nest there, except possibly killdeer (Jackson and Jackson 2000). In a study in southern Quebec, species richness and bird abundance were both greater in the adjacent habitats than in the road rights-of-way (Belanger et al. 2006). Given that roadside nesting species tend to be open country birds, roads that run through closed forest habitat probably have fewer breeding birds using the roadside than those running through open habitat. Exceptions to this general rule are probably habitat generalists (e.g., song sparrow) and species that use woodland edges such as Mourning Dove and Indigo Bunting. In an attempt to address this issue, we decided to incorporate habitat quality into the analysis. For each focal species, we categorized roadside habitat as either moderate or poor quality, based on the surrounding habitat type and the habitat preferences of each species. Sources for species habitat information were Peck and James $(1983,1987)$, Birds of North America online, and the CBBC database (Kennedy et al. 1999). Each category was then assigned a multiplier so we could discount nest density values to account for the habitat quality factor. The multipliers we used were 0.5 for moderate quality habitat and 0.1 for poor quality habitat. For example, if the density of savannah sparrow nests in preferred habitat was 1 per hectare, then our analysis would use half of that density for roads running through open country and one tenth of that density for roads running through forests. These multiplier values should be considered placeholders; an attempt to validate/revise them with research and expert opinion still needs to be made.

Additionally, we have allowed for some variation in roadside habitat type in our analysis. For example, a road running through open country might have a right-of-way composed mostly of grasses and herbs, whereas a road running through a forest may tend to have more of a woody component.

The combinations of habitat type and quality used for each focal species are listed in Table 3. 
Table 3. Combinations of habitat type and quality for each focal species. Surrounding Habitat is the habitat through which the roadside runs, and Right-of-Way Habitat is the habitat assumed to be available to nesting birds along the roadside. Habitat Quality Multipliers are used in the analysis to discount nest densities in non-preferred habitats.

\begin{tabular}{|c|c|c|}
\hline Surrounding Habitat & Right-of-Way Habitat (Habitat Quality) & $\begin{array}{l}\text { Habitat Quality } \\
\text { Multipliers }\end{array}$ \\
\hline Forest & $50 \%$ grass (poor), $50 \%$ shrub (poor) & $0.1,0.1$ \\
\hline Grassland & $100 \%$ grass (moderate) & no discount \\
\hline Shrubland & $50 \%$ grass (moderate), $50 \%$ shrub (moderate) & no discount \\
\hline Wetland & $50 \%$ grass (moderate), $50 \%$ wetland (moderate) & no discount \\
\hline \multicolumn{3}{|l|}{ Savannah Sparrow } \\
\hline Surrounding Habitat & Habitat of Road Right-of-Way (Habitat Quality) & $\begin{array}{l}\text { Habitat Quality } \\
\text { Multipliers }\end{array}$ \\
\hline Forest & $50 \%$ grass (poor), $50 \%$ shrub (poor) & $0.1,0.1$ \\
\hline Grassland & $100 \%$ grass (moderate) & 0.5 \\
\hline Shrubland & $50 \%$ grass (moderate), $50 \%$ shrub (poor) & $0.5,0.1$ \\
\hline Wetland & $50 \%$ grass (moderate), $50 \%$ wetland (poor) & $0.5,0.1$ \\
\hline \multicolumn{3}{|l|}{ Song Sparrow } \\
\hline Surrounding Habitat & Habitat of Road Right-of-Way (Habitat Quality) & $\begin{array}{l}\text { Habitat Quality } \\
\text { Multipliers }\end{array}$ \\
\hline Forest & $50 \%$ grass (moderate), $50 \%$ shrub (moderate) & $0.5,0.5$ \\
\hline Grassland & $100 \%$ grass (poor) & 0.1 \\
\hline Shrubland & $50 \%$ grass (moderate), $50 \%$ shrub (moderate) & $0.5,0.5$ \\
\hline Wetland & $50 \%$ grass (poor), $50 \%$ wetland (poor) & $0.1,0.1$ \\
\hline \multicolumn{3}{|c|}{ Clay-Colored Sparrow } \\
\hline Surrounding Habitat & Habitat of Road Right-of-Way (Habitat Quality) & $\begin{array}{l}\text { Habitat Quality } \\
\text { Multipliers }\end{array}$ \\
\hline Forest & $50 \%$ grass (poor), $50 \%$ shrub (poor) & $0.1,0.1$ \\
\hline Grassland & $100 \%$ grass (poor) & 0.1 \\
\hline Shrubland & $50 \%$ grass (moderate), $50 \%$ shrub (moderate) & $0.5,0.5$ \\
\hline Wetland & $50 \%$ grass (poor), $50 \%$ wetland (poor) & $0.1,0.1$ \\
\hline \multicolumn{3}{|l|}{ Vesper Sparrow } \\
\hline Surrounding Habitat & Habitat of Road Right-of-Way (Habitat Quality) & $\begin{array}{l}\text { Habitat Quality } \\
\text { Multipliers }\end{array}$ \\
\hline Forest & $50 \%$ grass (poor), $50 \%$ shrub (poor) & $0.1,0.1$ \\
\hline Grassland & $100 \%$ grass (moderate) & 0.5 \\
\hline Shrubland & $50 \%$ grass (moderate), $50 \%$ shrub (moderate) & $0.5,0.5$ \\
\hline Wetland & $50 \%$ grass (poor), $50 \%$ wetland (poor) & $0.1,0.1$ \\
\hline \multicolumn{3}{|l|}{ Mallard } \\
\hline Surrounding Habitat & Habitat of Road Right-of-Way (Habitat Quality) & $\begin{array}{l}\text { Habitat Quality } \\
\text { Multipliers }\end{array}$ \\
\hline Forest & $50 \%$ grass (poor), $50 \%$ shrub (poor) & $0.1,0.1$ \\
\hline Grassland & $100 \%$ grass (moderate) & 0.5 \\
\hline Shrubland & $50 \%$ grass (moderate), $50 \%$ shrub (moderate) & $0.5,0.5$ \\
\hline Wetland & $50 \%$ grass (moderate), $50 \%$ wetland (moderate) & $0.5,0.5$ \\
\hline
\end{tabular}




\subsection{Obtaining Information ABOUt AREA Disturbed}

The task of estimating area disturbed by mowing and/or brushing required a number of inputs. These included:

- total number of kilometers of roads by province/territory

- total number of kilometers of roads within the breeding ranges of each focal species in each province/territory

- total number of kilometers of roads in each of 4 general land cover types (forest, grassland, shrubland, wetland) within the breeding range of each focal species - for determining how to apply the habitat quality multipliers to nest density values

- total number of hectares mowed and/or brushed in each province/territory

- timing information for the disturbance

\section{GIS Analysis}

A GIS analysis was needed to obtain the required information about the extent of roads in each province and habitat type, and within each focal species breeding range.

The first step in this process was identifying an efficient, electronic source for the extent of Canada's road network. This source was the National Road Network of Canada (NRNC), available free of charge via the GeoBase web portal. The NRNC is the responsibility of Natural Resources Canada, and contains current, accurate geospatial data about Canada's roads. The NRNC is maintained under a federal-provincial-territorial-municipal agreement, and updates occur at least once per year.

The NRNC GIS layer included some road classes that were unlikely to result in incidental take for various reasons, and these were excluded from the analysis. The excluded road classes were: 1) winter roads; 2) local streets and back alleyways; and 3) rapid transit lanes for buses. Winter roads were excluded because the winter season is out of scope for our estimate of take. For the other excluded classes, we felt that their rights-of-way would either be too regularly maintained to be viable habitat for nesting birds (e.g., weekly mowing of rights-of-way by private property owners as in a subdivision), or they would be sidewalks (e.g., urban areas). The road classes that we did include in the analysis are listed in Table 4 - these are the roads that we considered likely to be maintained by government resources. 
Table 4. Road classes included in the GIS analysis for kilometers of road in each province/territory.

\begin{tabular}{|c|c|c|}
\hline Code & Road Class & Description \\
\hline 1 & Freeway & $\begin{array}{l}\text { An unimpeded, high speed controlled access thoroughfare for } \\
\text { through traffic with typically no at grade intersections, usually with no } \\
\text { property access or direct access and which is accessed by a Ramp. } \\
\text { Pedestrians prohibited. }\end{array}$ \\
\hline 2 & Expressway / Highway & $\begin{array}{l}\text { A high-speed thoroughfare with a combination of controlled access } \\
\text { intersections at any grade. }\end{array}$ \\
\hline 3 & Arterial & A major thoroughfare with medium to large traffic capacity. \\
\hline 4 & Collector & $\begin{array}{l}\text { A minor thoroughfare mainly used to access properties and to feed } \\
\text { traffic with right of way. }\end{array}$ \\
\hline 9 & Ramp & $\begin{array}{l}\text { A system of interconnecting roadways providing for the controlled } \\
\text { movement between two or more roadways. }\end{array}$ \\
\hline 10 & Resource / Recreation & $\begin{array}{l}\text { A narrow passage whose primary function is to provide access for } \\
\text { resource extraction and may also serve in providing public access to } \\
\text { the backcountry. }\end{array}$ \\
\hline 12 & Service Lane & $\begin{array}{l}\text { A stretch of road permitting vehicles to come to a stop along a } \\
\text { Freeway or Highway. Scale, service lane, emergency lane, lookout } \\
\text { and rest area. }\end{array}$ \\
\hline
\end{tabular}

Habitat types for all of Canada were also available from the GeoBase web portal, in the form of 250 separate land cover shapefiles. This land cover information comes from Landsat 5 and Landsat 7 ortho-images produced by the Canadian Forest Service (CFS) for the forested areas, by Agriculture and Agri-Food Canada (AAFC) for the agricultural areas, and by the Canadian Centre of Remote Sensing (CCRS) for the northern territories. The land cover classification system used is a harmonization of existing systems used by these three agencies, and is summarized at http://www.geobase.ca/doc/specs/pdf/GeoBase_LCC2000V_Harmonization_Legend.pdf. The harmonized classification contains 43 land cover types, 10 of which are un-vegetated. From these, we created 4 general habitat types - Forest, Shrubland, Grassland and Wetland - that captured $91 \%$ of vegetated land cover classes in Canada (Table 5).

Table 5. General habitat types used in the analysis and the land cover classes that comprise them; these 4 Habitat Types represent $91 \%$ of the vegetated land covers classes in Canada.

\begin{tabular}{|l|l|c|}
\hline Habitat Types & Land Cover Classes & Legend Code \\
\hline Grassland & Herb & 100 \\
& Tussock graminoid tundra & 101 \\
& Grassland & 110 \\
& Cultivated Agricultural Land & 121 \\
& Annual Crops & 122 \\
& Perennial Crops and Pastures & 123 \\
\hline Shrubland & Shrubland & 50 \\
& Shrub- Tall & 51 \\
& Shrub - Low & 52 \\
& Prostrate dwarf shrub & 53 \\
& Moist to dry non-tussock graminoid/shrub tundra & 103 \\
& Dry graminoid prostrate dwarf shrub & 104 \\
\hline Forest & Forest/Trees & 200 \\
& Coniferous & 210 \\
& Coniferous- Dense & 211 \\
& Coniferous - Open & 212 \\
\hline
\end{tabular}




\begin{tabular}{|l|l|c|}
\hline Habitat Types & Land Cover Classes & Legend Code \\
\hline & Coniferous - Sparse & 213 \\
& Broadleaf & 220 \\
& Broadleaf - Dense & 221 \\
& Broadleaf - Open & 222 \\
& Broadleaf - Sparse & 223 \\
& Mixedwood & 230 \\
& Mixedwood - Dense & 231 \\
& Mixedwood - Open & 232 \\
& Mixedwood - Sparse & 233 \\
\hline Wetland & Wetland & 80 \\
& Wetland Treed & 81 \\
& Wetland Shrub & 82 \\
& Wetland Herb & 83 \\
& Wet Sedge & 102 \\
\hline
\end{tabular}

The final input for the GIS analysis was information about the breeding ranges of each focal species on the list from NatureServe 3.0 (http://www.natureserve.org/getData/birdMaps.jsp).

The total number of kilometers of maintained road (filtered as described above) in each province/territory is shown in Table 6.

Table 6. The extent of roads in Canada, by province and territory.

\begin{tabular}{lrr}
\hline Province/Territory & $\begin{array}{r}\text { Total Maintenance } \\
\text { Road Length } \mathbf{( k m )}\end{array}$ & $\begin{array}{r}\text { Total Road } \\
\text { Length (km) }\end{array}$ \\
\hline BC & 32,669 & 77,248 \\
Yukon & 3,660 & 6,043 \\
NW Territories & 2,773 & 5,358 \\
Alberta & 221,211 & 231,567 \\
Saskatchewan & 239,662 & 250,455 \\
Manitoba & 78,610 & 87,864 \\
Ontario & 99,591 & 234,769 \\
Quebec & 113,965 & 146,586 \\
New Brunswick & 26,731 & 31,740 \\
Nova Scotia & 21,741 & 45,707 \\
PEI & 3,500 & 6,708 \\
Newfoundland/Labrador & 16,143 & 19,632 \\
Nunavut & 405 & 916 \\
\hline Total & 860,661 & $1,144,596$ \\
\hline Iaintenance road length reflects the exclusion of
\end{tabular}

1 Maintenance road length reflects the exclusion of urban and suburban roads.

The unfiltered total extent of roads in Canada, based on the NRNC data, is approximately $1.1 \mathrm{M}$ $\mathrm{km}$. This agrees reasonably well with Forman et al. (2003:38), who reported a total of 902,000 $\mathrm{km}$ of 2-lane equivalent roadway in Canada for the year 2000 . Of these, $574,000 \mathrm{~km}$ are unpaved, $312,000 \mathrm{~km}$ are paved, and $16,000 \mathrm{~km}$ are freeways. The process of filtering out the surburban/urban and rapid transit road classes has reduced total road extent for each province and territory; in some cases, the reduction is quite substantial (e.g. road length in Ontario was reduced from 235,000 to $99,000 \mathrm{~km}$ ). This may not be an unreasonable result, given the extensive road networks in the many cities and towns of southern Ontario. Additionally, our filtering resulted in a total maintenance road length for British Columbia that was surprisingly 
small $(<33,000 \mathrm{~km})$. However, contacts at the British Columbia Ministry of Transportation, verified this value as reasonable since they maintain about $47,000 \mathrm{~km}$ of roads including some suburban/urban.

The number of kilometers of maintenance road (filtered as described above) within each focal species breeding range is shown in Table 7.

Table 7. Number of kilometers of road (filtered) within the breeding range for 6 focal species.

\begin{tabular}{lrrrrrr} 
& \multicolumn{7}{c}{ Kilometers of Road within Range } \\
\cline { 2 - 7 } & \multicolumn{1}{c}{ KILL } & \multicolumn{1}{c}{ SASP } & \multicolumn{1}{c}{ SOSP } & CCSP & \multicolumn{1}{c}{ VESP } & MALL \\
\hline BC & 16,996 & 17,361 & 17,667 & 6,760 & 10,519 & 17,667 \\
Yukon & 756 & 2,436 & 702 & 0 & 0 & 2,436 \\
NW Territories & 307 & 337 & 264 & 253 & 0 & 339 \\
Alberta & 183,870 & 183,870 & 183,870 & 183,870 & 183,804 & 183,870 \\
Saskatchewan & 210,538 & 210,538 & 210,538 & 210,508 & 209,059 & 210,538 \\
Manitoba & 47,392 & 47,392 & 47,392 & 47,081 & 45,847 & 47,359 \\
Ontario & 66,759 & 66,769 & 66,759 & 53,240 & 63,503 & 66,759 \\
Quebec & 63,500 & 74,158 & 63,333 & 13,610 & 50,024 & 70,320 \\
New Brunswick & 16,693 & 16,724 & 16,724 & 0 & 10,463 & 16,611 \\
Nova Scotia & 8,079 & 15,411 & 15,411 & 0 & 8,957 & 12,567 \\
PEI & 2,764 & 2,764 & 2,764 & 0 & 2,764 & 0 \\
Newfoundland/Labrador & 0 & 9,714 & 1,401 & 0 & 0 & 0 \\
Nunavut & 0 & 31 & 0 & 0 & 0 & 0 \\
\hline
\end{tabular}

\section{Researching Area Disturbed}

Information about area disturbed was collected from provincial and territorial transportation ministries (contacts are provided in Appendix A); these agencies managed most of the road kilometers in the country. Some jurisdictions did not provide data, so it was necessary to generate estimates for them based on information obtained from the responding jurisdictions. For each province/territory that provided disturbance data, we calculated the proportion of total roadside hectares that were mowed or brushed. We then averaged the proportion maintained for the responding provinces/territories, and applied this proportion to the total roadside hectares for all non-respondent jurisdictions to derive an estimate of area maintained for them. In terms of the timing of maintenance operations for non-respondent jurisdictions, we assumed an even distribution of effort between June and August inclusive, unless information obtained from interviews with transportation contacts indicated otherwise.

The road maintenance inputs used in the simulation model are shown in Appendix $\mathrm{C}$. These values can easily be updated in the model if/when better quality data become available.

\subsection{Calculating Incidental TAKe}

Disturbance data were expressed as total area by province/territory (analysis region). Area disturbed within each region for each focal species was derived by overlaying the digital road data with the digital bird distribution data. This approach assumes an even distribution of area disturbed across all roads within each province/territory.

The mortality rate for eggs and nestlings exposed to maintenance operations was assumed to be $100 \%$ for all focal species; adult mortality was assumed to be $0 \%$ for all focal species except waterfowl (after Tews et al. 2009). A literature review provided in Tews et al. (2009) noted that some proportion of nesting adults of most waterfowl species are subject to mortality from 
mowing. We have used a mortality rate of $23.5 \%$ (i.e. 23.5 adults killed per 100 nests destroyed), consistent with Tews.

For each focal species (except mallard), the total number of birds killed by maintenance operations was calculated by multiplying the number of eggs/nestlings present on a given day (after adjustments for habitat quality) by the number of hectares disturbed on the same day and then summing these results across all days within the breeding range and within each province/territory. For mallards, the number of adults killed, based on nest density, was tallied separately from the number of eggs/nestlings affected.

For each focal species, we calculated the proportion of birds killed relative to published estimates of total population size for each province/territory. Population estimates were obtained from the Partners in Flight/Breeding Bird Survey online database (http://rmbo.org/pif db/laped) (see Blancher et al. 2007) for the landbirds (savannah sparrow, song sparrow, clay-colored sparrow and vesper sparrow) and from Blancher (2002) for killdeer. For Mallards, population estimates were obtained for the Prairie Provinces from provincial North American Waterfowl Management Plan (NAWMP) implementation plans prepared under the Prairie Habitat Joint Venture (PHJV) (Manitoba Implementation Plan Committee 2008, Saskatchewan NAWMP Technical Committee 2008, Alberta NAWMP Partnership Management Committee 2008). Data used were the 2007 10-year average breeding population for the area covered by the PHJV for each province. Comparable data were not available for other provinces. For Ontario, Quebec and the Maritime provinces, data were provided by the Canadian Wildlife Service (J. Hughes pers. comm.) based on stratified surveys from areal and ground reconnaissance last completed in 2005. Data for British Columbia and the territories could not be obtained. These proportions were used to calculate incidental take for all non-focal roadside species (as per Tews et al. 2009). For example, if an average of $0.1 \%$ of savannah sparrows in Ontario were calculated to be lost to roadside maintenance operations, we applied the same percent loss to the non-focal members of the group in that province. This approach assumes that take for non-focal species is proportionately similar to take for focal species. 


\section{RESULTS}

We calculated that approximately 494,000 to 1.525 million birds are killed annually (median value approx. 861,000$)$ by maintenance operations. Most $(95 \%)$ of these are American robins (but see discussion in Section 5 explaining the calculated robin mortality). On a proportional basis, incidental take was less than $1 \%$ of the total population for all focal species ${ }^{2}$ at the national scale.

For the 6 focal species, the estimated number of birds (adults, eggs and nestlings) lost each year in Canada as a result of roadside maintenance activities is presented for each province/territory in Table 8, and for all of Canada in Table 9. Of the focal species, the highest level of calculated take is for savannah sparrow (approx. 9,300), and the least for clay-coloured sparrow (approx. 1,090). No obvious trends are apparent, as levels of take are largely a function of the nest densities in the various provinces, affinity for roadside habitat, and nesting ecology (i.e. dates of egg-laying, incubation times, etc.) as described in Section 3.4 and in considerable detail in Appendix B.

Estimates extrapolated to the non-focal roadside nesting species are provided in Table 10. Totals are summarised in Table 11. For the non-focal species the highest level of estimate mortality is, for American robin, and the least for lark sparrow (median value 7).

Table 8. Incidental take for 6 focal species by province/territory. Mortality $=$ number of birds (adults, nestlings, eggs) killed by maintenance operations. POP= population estimates- from Partners in Flight Landbird Database for all birds except mallards; for mallards population estimates were taken from the Habitat Joint Ventures as explained in text. \%Pop = the percent of the population killed, and is used for calculation of incidental take of non-focal species in each group. The median (50\%), and the 50th percentile and the 95\% percentile interval from 1000 simulations are reported, where LB (lower bound) $=2.5$ percentile and UB (upper bound) $=97.5$ percentile. For all species except mallard, mortality is eggs and nestlings only; for mallards mortality of adults is indicated separately from mortality of eggs and nestlings.

\begin{tabular}{|c|c|c|c|c|c|c|c|c|}
\hline \multirow[t]{2}{*}{ Species } & \multirow[t]{2}{*}{ Prov/Terr } & \multicolumn{3}{|c|}{ Mortality (percentiles) } & \multirow[t]{2}{*}{ POP } & \multicolumn{3}{|c|}{ \%Pop (Mortality/POP*100) } \\
\hline & & $50 \%$ & LB $=2.5 \%$ & UB $=97.5 \%$ & & Estimate & LB & UB \\
\hline \multirow[t]{13}{*}{ Killdeer $^{1}$} & $A B$ & 27 & 20 & 38 & & & & \\
\hline & $\mathrm{BC}$ & 7 & 5 & 10 & & & & \\
\hline & MB & 129 & 106 & 160 & & & & \\
\hline & NB & 0 & 0 & 0 & & & & \\
\hline & $\mathrm{NL}$ & 0 & 0 & 0 & & & & \\
\hline & NS & 0 & 0 & 0 & & & & \\
\hline & $\mathrm{NU}^{2}$ & 0 & 0 & 0 & & & & \\
\hline & NWT & 0 & 0 & 0 & & & & \\
\hline & ON & 580 & 341 & 989 & & & & \\
\hline & PEI & 0 & 0 & 0 & & & & \\
\hline & $\mathrm{QC}$ & 0 & 0 & 0 & & & & \\
\hline & SK & 1283 & 803 & 2019 & & & & \\
\hline & YT & 0 & 0 & 0 & & & & \\
\hline Total & & 2026 & 1275 & 3216 & & & & \\
\hline Savannah & $A B$ & 1296 & 555 & 2854 & $8,000,000$ & 0.01620 & 0.00694 & 0.03568 \\
\hline \multirow[t]{2}{*}{ Sparrow } & $\mathrm{BC}$ & 25 & 13 & 48 & $3,000,000$ & 0.00083 & 0.00043 & 0.00160 \\
\hline & $\mathrm{MB}$ & 1982 & 810 & 4605 & $7,000,000$ & 0.02831 & 0.01157 & 0.06579 \\
\hline
\end{tabular}

${ }^{2}$ Because we used proportion of the total population that was taken for focal species to estimate take for non-focal species, we can't make any statements about the proportional take for non-focal species. 


\begin{tabular}{|c|c|c|c|c|c|c|c|c|}
\hline \multirow[t]{2}{*}{ Species } & \multirow[t]{2}{*}{ Prov/Terr } & \multicolumn{3}{|c|}{ Mortality (percentiles) } & \multirow[t]{2}{*}{ POP } & \multicolumn{3}{|c|}{ \%Pop (Mortality/POP*100) } \\
\hline & & $50 \%$ & $\mathrm{LB}=2.5 \%$ & UB $=97.5 \%$ & & Estimate & LB & UB \\
\hline & NB & 0 & 0 & 0 & 200,000 & 0 & 0 & 0 \\
\hline & NL & 0 & 0 & 0 & $1,300,000$ & 0 & 0 & 0 \\
\hline & NS & 2 & 1 & 4 & 190,000 & 0.00105 & 0.00053 & 0.00211 \\
\hline & $N U^{2}$ & 0 & 0 & 0 & & & & \\
\hline & NWT & 7 & 4 & 13 & 300,000 & 0.00233 & 0.00133 & 0.00433 \\
\hline & ON & 2566 & 1176 & 6324 & $6,000,000$ & 0.04277 & 0.01960 & 0.10540 \\
\hline & PEI & 0 & 0 & 0 & 80,000 & 0 & 0 & 0 \\
\hline & QC & 2173 & 1028 & 4789 & $3,000,000$ & 0.07243 & 0.03427 & 0.15963 \\
\hline & SK & 1248 & 758 & 1989 & $6,000,000$ & 0.02080 & 0.01263 & 0.03315 \\
\hline & YT & 7 & 4 & 14 & 700,000 & 0.00100 & 0.00057 & 0.00200 \\
\hline Total & & 9306 & 4349 & 20640 & & & & \\
\hline \multirow{13}{*}{$\begin{array}{l}\text { Song } \\
\text { Sparrow }\end{array}$} & $A B$ & 77 & 50 & 121 & $4,000,000$ & 0.00193 & 0.00125 & 0.00303 \\
\hline & $\mathrm{BC}$ & 50 & 15 & 162 & $3,000,000$ & 0.00167 & 0.00050 & 0.00540 \\
\hline & MB & 415 & 257 & 656 & $3,000,000$ & 0.01383 & 0.00857 & 0.02187 \\
\hline & NB & 0 & 0 & 0 & 690,000 & 0 & 0 & 0 \\
\hline & $\mathrm{NL}$ & 0 & 0 & 0 & 60,000 & 0 & 0 & 0 \\
\hline & NS & 41 & 23 & 74 & $1,000,000$ & 0.00410 & 0.00230 & 0.00740 \\
\hline & $\mathrm{NU}^{2}$ & 0 & 0 & 0 & & & & \\
\hline & NWT & 0 & 0 & 0 & 40,000 & 0 & 0 & 0 \\
\hline & ON & 2033 & 800 & 5016 & $4,000,000$ & 0.05083 & 0.02000 & 0.12540 \\
\hline & PEI & 3 & 2 & 4 & 170,000 & 0 & 0 & 0 \\
\hline & $\mathrm{QC}$ & 63 & 47 & 82 & $4,000,000$ & 0.00158 & 0.00118 & 0.00205 \\
\hline & SK & 1493 & 902 & 2355 & $4,000,000$ & 0.03733 & 0.02255 & 0.05888 \\
\hline & YT & 0 & 0 & 0 & 70,000 & 0.00000 & 0.00000 & 0.00000 \\
\hline Total & & 4175 & 2096 & 8470 & & & & \\
\hline \multirow{13}{*}{$\begin{array}{l}\text { Clay- } \\
\text { colored } \\
\text { Sparrow }\end{array}$} & $A B$ & 492 & 161 & 1543 & $7,000,000$ & 0.00703 & 0.00230 & 0.02204 \\
\hline & $\mathrm{BC}$ & 147 & 50 & 400 & $1,600,000$ & 0.00919 & 0.00313 & 0.02500 \\
\hline & MB & 99 & 48 & 200 & $2,700,000$ & 0.00367 & 0.00178 & 0.00741 \\
\hline & NB & 0 & 0 & 0 & - & & & \\
\hline & $\mathrm{NL}$ & 0 & 0 & 0 & - & & & \\
\hline & NS & 0 & 0 & 0 & - & & & \\
\hline & $\mathrm{NU}^{2}$ & 0 & 0 & 0 & & & & \\
\hline & NWT & 0 & 0 & 0 & 600,000 & 0 & 0 & 0 \\
\hline & ON & 151 & 88 & 265 & 60,000 & 0.25167 & 0.14667 & 0.44167 \\
\hline & PEI & 0 & 0 & 0 & - & & & \\
\hline & $\mathrm{QC}$ & 104 & 60 & 178 & 3,000 & 3.46667 & 2.00000 & 5.93333 \\
\hline & SK & 95 & 55 & 171 & $7,000,000$ & 0.00136 & 0.00079 & 0.00244 \\
\hline & YT & 0 & 0 & 0 & 19,000 & 0.00000 & 0.00000 & 0.00000 \\
\hline Total & & 1088 & 462 & 2757 & & & & \\
\hline \multirow{13}{*}{$\begin{array}{l}\text { Vesper } \\
\text { Sparrow }\end{array}$} & $A B$ & 296 & 156 & 492 & $3,000,000$ & 0.00987 & 0.00520 & 0.01640 \\
\hline & $B C$ & 0 & 0 & 0 & $1,300,000$ & 0 & 0 & 0 \\
\hline & MB & 0 & 0 & 0 & $1,100,000$ & 0 & 0 & 0 \\
\hline & NB & 0 & 0 & 0 & 500 & 0 & 0 & 0 \\
\hline & $\mathrm{NL}$ & 0 & 0 & 0 & - & & & \\
\hline & NS & 0 & 0 & 0 & 1,400 & 0 & 0 & 0 \\
\hline & $\mathrm{NU}^{2}$ & 0 & 0 & 0 & & & & \\
\hline & NWT & 0 & 0 & 0 & - & & & \\
\hline & ON & 779 & 447 & 1388 & 170,000 & 0.45824 & 0.26294 & 0.81647 \\
\hline & PEI & 0 & 0 & 0 & 700 & 0 & 0 & 0 \\
\hline & $\mathrm{QC}$ & 407 & 244 & 670 & 120,000 & 0.33917 & 0.20333 & 0.55833 \\
\hline & SK & 1571 & 958 & 2647 & $5,000,000$ & 0.03142 & 0.01916 & 0.05294 \\
\hline & YT & 0 & 0 & 0 & - & & & \\
\hline Total & & 3053 & 1805 & 5197 & & & & \\
\hline Mallard $^{3}$ & $A B$ & 1346 & 608 & 3014 & $1,200,000$ & 0.11217 & .0 .5067 & 0.25117 \\
\hline Eggs and & $\mathrm{BC}$ & 9 & 7 & 11 & & & & \\
\hline \multirow[t]{2}{*}{ Nestlings } & MB & 805 & 535 & 1237 & 445,000 & 0.18090 & 0.12022 & 0.27780 \\
\hline & NB & 23 & 13 & 38 & 5,000 & 0.46000 & 0.26000 & 0.76000 \\
\hline
\end{tabular}




\begin{tabular}{|c|c|c|c|c|c|c|c|c|}
\hline \multirow[t]{2}{*}{ Species } & \multirow[t]{2}{*}{ Prov/Terr } & \multicolumn{3}{|c|}{ Mortality (percentiles) } & \multirow[t]{2}{*}{ POP } & \multicolumn{3}{|c|}{ \%Pop (Mortality/POP*100) } \\
\hline & & $50 \%$ & $\mathrm{LB}=2.5 \%$ & $U B=97.5 \%$ & & Estimate & LB & UB \\
\hline & $\mathrm{NL}$ & 0 & 0 & 0 & 0 & 0 & 0 & 0 \\
\hline & NS & 44 & 24 & 78 & 4,000 & 1.1000 & 0.6000 & 1.95000 \\
\hline & NU & 0 & 0 & 0 & & & & \\
\hline & NWT & 2 & 1 & 3 & & & & \\
\hline & ON & 805 & 446 & 1526 & 320,000 & 0.25156 & 0.13937 & 0.47688 \\
\hline & PEI & 0 & 0 & 0 & 3,000 & 0 & 0 & 0 \\
\hline & $\mathrm{QC}$ & 759 & 418 & 1325 & 93,600 & 0.81090 & 0.44659 & 1.41560 \\
\hline & SK & 1707 & 1046 & 2780 & $2,000,000$ & 0.08535 & 0.05230 & 0.13900 \\
\hline & YT & 1 & 1 & 2 & & & & \\
\hline Total & & 5501 & 3099 & 10014 & & & & \\
\hline \multirow{13}{*}{$\begin{array}{l}\text { Mallard } \\
\text { Adults }\end{array}$} & $A B$ & 36 & 16 & 82 & $1,200,000$ & 0.003 & 0.00133 & 0.00683 \\
\hline & $\mathrm{BC}$ & 0 & 0 & 0 & & & & \\
\hline & $\mathrm{MB}$ & 22 & 14 & 33 & 445,000 & 0.00494 & 0.00315 & 0.00741 \\
\hline & NB & 1 & 0 & 1 & 5,000 & 0.02000 & 0 & 0.02000 \\
\hline & $\mathrm{NL}$ & 0 & 0 & 0 & 0 & 0 & 0 & 0 \\
\hline & NS & 1 & 1 & 1 & 4,000 & 0.02500 & 0.025000 & 0.05000 \\
\hline & $\mathrm{NU}$ & 0 & 0 & 0 & & & & \\
\hline & NWT & 0 & 0 & 0 & & & & \\
\hline & ON & 22 & 12 & 41 & 320,000 & 0.00688 & 0.00375 & 0.01281 \\
\hline & PEI & 0 & 0 & 0 & 3,000 & 0 & 0 & 0 \\
\hline & $\mathrm{QC}$ & 21 & 12 & 36 & 93,600 & 0.02244 & 0.01282 & 0.38462 \\
\hline & SK & 46 & 29 & 75 & $2,000,000$ & 0.00230 & 0.00145 & 0.00375 \\
\hline & $\mathrm{YT}$ & 0 & 0 & 0 & & & & \\
\hline Total & & 151 & 85 & 273 & & & & \\
\hline \multicolumn{2}{|c|}{ Focal Species Total } & 25,298 & 13,170 & 50,564 & & & & \\
\hline
\end{tabular}

Because killdeer is the only member of its group, there is no need to calculate a value for \%Pop to use in calculating the incidental take of non-focal species in the same group.

2 Population estimates from Partners in Flight combine Nunavut and the Northwest Territories, so estimates of incidental take for these two territories have been combined in order to calculate \%Pop.

3 Mallard population estimates were not available for BC, Nunavut, NWT and Yukon.

Table 9. Incident take for 6 focal species for all of Canada. Total Take $=$ number of birds killed annually in Canada. \%Tot $=$ percent of the total annual take. Median, lower bound (LB=2.5 percentile) and upper bound (UB=97.5 percentile) values are reported.

\begin{tabular}{|c|c|c|c|}
\hline & \multicolumn{3}{|c|}{ Total Take (\%Tot) } \\
\hline & Median & LB & UB \\
\hline Killdeer & $2,026(8.1)$ & $1,275(9.7)$ & $3,216(6.4)$ \\
\hline Savannah Sparrow & $9,306(36.7)$ & $4,349(33.0)$ & $20,640(40.8)$ \\
\hline Song Sparrow & 4,175 (16.5) & 2,096 (15.9) & $8,470(16.8)$ \\
\hline Clay-colored Sparrow & $1,088(4.3)$ & $462(3.5)$ & $2,757(5.4)$ \\
\hline Vesper Sparrow & $3,053(12.1)$ & $1,805(13.7)$ & $5,197(10.3)$ \\
\hline Mallard (eggs/nestlings) & $5,501(21.7)$ & $3,099(23.5)$ & $10,014(19.8)$ \\
\hline Mallard (adults) & $149(0.6)$ & $84(0.6)$ & $270(0.5)$ \\
\hline Total & $25,298(100)$ & $13,170(100)$ & $50,564(100)$ \\
\hline
\end{tabular}


Table 10. Incidental take for 12 non-focal species by province/territory. \%Pop focal $=$ the percent of the focal species population killed (from Table 8). Pop Est - population estimates. Estimated take (Mortality) = Number of birds (adults, nestlings, eggs) killed by maintenance operations, calculated as proportion lost (\%Pop focal/100) multiplied by the population size of the non-focal species. $L B=$ lower bound on the estimate; UB = upper bound on the estimate. For example: for American Goldfinch in Alberta, we estimate $(0.0162 / 100) \star 840,000=136.08$ birds as the median take. Note that separate estimates are provided for waterfowl eggs and nestlings, and adults.

\begin{tabular}{|c|c|c|c|c|c|c|c|c|c|}
\hline \multirow[b]{2}{*}{ Non-focal Species } & \multirow[b]{2}{*}{$\begin{array}{l}\text { Focal } \\
\text { species }\end{array}$} & \multicolumn{4}{|c|}{ \%Pop focal } & \multirow[b]{2}{*}{$\begin{array}{l}\text { Pop. } \\
\text { Est. }\end{array}$} & \multicolumn{3}{|c|}{$\begin{array}{l}\text { Estimated take (Mortality) } \\
\text { Non-focal species }\end{array}$} \\
\hline & & Region & Median & LB & UB & & Median & LB & UB \\
\hline \multirow[t]{14}{*}{ American Goldfinch } & Savannah & & & & & & & & \\
\hline & Sparrow & $A B$ & 0.0162 & 0.00694 & 0.03568 & 840,000 & 136 & 58 & 300 \\
\hline & & $\mathrm{BC}$ & 0.00083 & 0.00043 & 0.0016 & 400,000 & 3 & 2 & 6 \\
\hline & & MB & 0.02831 & 0.01157 & 0.06579 & $1,000,000$ & 283 & 116 & 658 \\
\hline & & NB & 0 & 0 & 0 & 300,000 & 0 & 0 & 0 \\
\hline & & NL & 0 & 0 & 0 & 40,000 & 0 & 0 & 0 \\
\hline & & NS & 0.00105 & 0.00053 & 0.00211 & 500,000 & 5 & 3 & 11 \\
\hline & & NU & & & & - & & & \\
\hline & & NWT & 0.00233 & 0.00133 & 0.00433 & - & & & \\
\hline & & ON & 0.04277 & 0.0196 & 0.1054 & $1,700,000$ & 727 & 333 & 1792 \\
\hline & & PEI & 0 & 0 & 0 & 30,000 & 0 & 0 & 0 \\
\hline & & $\mathrm{QC}$ & 0.07243 & 0.03427 & 0.15963 & $1,800,000$ & 1304 & 617 & 2873 \\
\hline & & SK & 0.0208 & 0.01263 & 0.03315 & $1,800,000$ & 374 & 227 & 596.7 \\
\hline & & YT & 0.001 & 0.00057 & 0.002 & - & & & \\
\hline Total & & & & & & & 2,832 & 1,356 & 5,640 \\
\hline \multirow[t]{14}{*}{$\begin{array}{l}\text { Eastern } \\
\text { Meadowlark }\end{array}$} & $\begin{array}{l}\text { Savannah } \\
\text { Sparrow }\end{array}$ & & & & & & & & \\
\hline & & $A B$ & 0.0162 & 0.00694 & 0.03568 & - & & & \\
\hline & & $B C$ & 0.00083 & 0.00043 & 0.0016 & - & & & \\
\hline & & MB & 0.02831 & 0.01157 & 0.06579 & - & & & \\
\hline & & NB & 0 & 0 & 0 & 300 & 0 & 0 & 0 \\
\hline & & NL & 0 & 0 & 0 & - & & & \\
\hline & & NS & 0.00105 & 0.00053 & 0.00211 & - & & & \\
\hline & & NU & & & & - & & & \\
\hline & & NWT & 0.00233 & 0.00133 & 0.00433 & - & & & \\
\hline & & ON & 0.04277 & 0.0196 & 0.1054 & 200,000 & 86 & 39 & 211 \\
\hline & & PEI & 0 & 0 & 0 & - & & & \\
\hline & & QC & 0.07243 & 0.03427 & 0.15963 & 60,000 & 43 & 21 & 96 \\
\hline & & SK & 0.0208 & 0.01263 & 0.03315 & - & & & \\
\hline & & YT & 0.001 & 0.00057 & 0.002 & - & & & \\
\hline Total & & & & & & & 129 & 60 & 307 \\
\hline \multirow[t]{2}{*}{$\begin{array}{l}\text { Western } \\
\text { Meadowlark }\end{array}$} & $\begin{array}{l}\text { Savannah } \\
\text { Sparrow }\end{array}$ & & & & & & & & \\
\hline & & $A B$ & 0.0162 & 0.00694 & 0.03568 & 810,000 & 131 & 56 & 289 \\
\hline
\end{tabular}




\begin{tabular}{|c|c|c|c|c|c|c|c|c|c|}
\hline \multirow[b]{2}{*}{ Non-focal Species } & \multirow[b]{2}{*}{$\begin{array}{l}\text { Focal } \\
\text { species }\end{array}$} & \multicolumn{4}{|c|}{ \%Pop focal } & \multicolumn{4}{|c|}{$\begin{array}{l}\text { Estimated take (Mortality) } \\
\text { Non-focal species }\end{array}$} \\
\hline & & Region & Median & LB & UB & $\begin{array}{l}\text { Pop. } \\
\text { Est. }\end{array}$ & Median & LB & UB \\
\hline & & $\mathrm{BC}$ & 0.00083 & 0.00043 & 0.0016 & 300,000 & 2 & 1 & 5 \\
\hline & & $\mathrm{MB}$ & 0.02831 & 0.01157 & 0.06579 & 380,000 & 108 & 44 & 250 \\
\hline & & NB & 0 & 0 & 0 & - & & & \\
\hline & & NL & 0 & 0 & 0 & - & & & \\
\hline & & NS & 0.00105 & 0.00053 & 0.00211 & - & & & \\
\hline & & NU & & & & - & & & \\
\hline & & NWT & 0.00233 & 0.00133 & 0.00433 & - & & & \\
\hline & & ON & 0.04277 & 0.0196 & 0.1054 & 6,000 & 3 & 1 & 6 \\
\hline & & PEI & 0 & 0 & 0 & - & & & \\
\hline & & QC & 0.07243 & 0.03427 & 0.15963 & - & & & \\
\hline & & SK & 0.0208 & 0.01263 & 0.03315 & $1,100,000$ & 229 & 139 & 365 \\
\hline & & YT & 0.001 & 0.00057 & 0.002 & - & & & \\
\hline Total & & & & & & & 473 & 241 & 915 \\
\hline \multirow[t]{14}{*}{ Mourning Dove } & Song & & & & & & & & \\
\hline & Sparrow & $A B$ & 0.00193 & 0.00125 & 0.00303 & 220,000 & 4 & 3 & 7 \\
\hline & & $\mathrm{BC}$ & 0.00167 & 0.0005 & 0.0054 & 180,000 & 3 & 1 & 10 \\
\hline & & $\mathrm{MB}$ & 0.01383 & 0.00857 & 0.02187 & $1,200,000$ & 166 & 103 & 262 \\
\hline & & NB & 0 & 0 & 0 & 140,000 & 0 & 0 & 0 \\
\hline & & $\mathrm{NL}$ & 0 & 0 & 0 & 400 & 0 & 0 & 0 \\
\hline & & NS & 0.0041 & 0.0023 & 0.0074 & 80,000 & 3 & 2 & 6 \\
\hline & & $\mathrm{NU}$ & & & & - & & & \\
\hline & & NWT & 0 & 0 & 0 & - & & & \\
\hline & & ON & 0.05083 & 0.02 & 0.1254 & $1,200,000$ & 610 & 240 & 1505 \\
\hline & & PEI & 0 & 0 & 0 & 5,000 & 0 & 0 & 0 \\
\hline & & $\mathrm{QC}$ & 0.00158 & 0.00118 & 0.00205 & 590,000 & 9 & 7 & 12 \\
\hline & & SK & 0.03733 & 0.02255 & 0.05888 & $1,700,000$ & 635 & 383 & 1001 \\
\hline & & YT & 0 & 0 & 0 & - & & & \\
\hline Total & & & & & & & 1,430 & 739 & 2,803 \\
\hline \multirow[t]{14}{*}{ Indigo Bunting } & Song & & & & & & & & \\
\hline & Sparrow & $A B$ & 0.00193 & 0.00125 & 0.00303 & - & & & \\
\hline & & $\mathrm{BC}$ & 0.00167 & 0.0005 & 0.0054 & - & & & \\
\hline & & MB & 0.01383 & 0.00857 & 0.02187 & 14,000 & 2 & 1 & 3 \\
\hline & & NB & 0 & 0 & 0 & - & & & \\
\hline & & $\mathrm{NL}$ & 0 & 0 & 0 & - & & & \\
\hline & & NS & 0.0041 & 0.0023 & 0.0074 & - & & & \\
\hline & & NU & & & & - & & & \\
\hline & & NWT & 0 & 0 & 0 & - & & & \\
\hline & & ON & 0.05083 & 0.02 & 0.1254 & 350,000 & 178 & 70 & 439 \\
\hline & & PEI & 0 & 0 & 0 & - & & & \\
\hline & & $\mathrm{QC}$ & 0.00158 & 0.00118 & 0.00205 & 90,000 & 1 & 1 & 2 \\
\hline & & SK & 0.03733 & 0.02255 & 0.05888 & - & & & \\
\hline & & YT & 0 & 0 & 0 & - & & & \\
\hline
\end{tabular}




\begin{tabular}{|c|c|c|c|c|c|c|c|c|c|}
\hline \multirow[b]{2}{*}{ Non-focal Species } & \multirow[b]{2}{*}{$\begin{array}{l}\text { Focal } \\
\text { species }\end{array}$} & \multicolumn{4}{|c|}{ \%Pop focal } & \multirow[b]{2}{*}{$\begin{array}{l}\text { Pop. } \\
\text { Est. }\end{array}$} & \multicolumn{3}{|c|}{$\begin{array}{l}\text { Estimated take (Mortality) } \\
\text { Non-focal species }\end{array}$} \\
\hline & & Region & Median & LB & UB & & Median & LB & UB \\
\hline \multirow[t]{14}{*}{ American Robin } & Clay- & & & & & & & & \\
\hline & & $A B$ & 0.00703 & 0.0023 & 0.02204 & 12000000 & 844 & 276 & 2645 \\
\hline & & $\mathrm{BC}$ & 0.00919 & 0.00313 & 0.025 & 30000000 & 2757 & 939 & 7500 \\
\hline & & $\mathrm{MB}$ & 0.00367 & 0.00178 & 0.00741 & 9000000 & 330 & 160 & 667 \\
\hline & & NB & 0.4671 & 0.269 & 0.804 & 3000000 & 14010 & 8070 & 24120 \\
\hline & & $\mathrm{NL}$ & $0.467^{1}$ & 0.269 & 0.804 & 9000000 & 42030 & 24210 & 72360 \\
\hline & & NS & $0.467^{1}$ & 0.269 & 0.804 & 3000000 & 14010 & 8070 & 24120 \\
\hline & & NU & 0 & 0 & 0 & 18000000 & 0 & 0 & 0 \\
\hline & & NWT & 0 & 0 & 0 & & 0 & 0 & 0 \\
\hline & & ON & 0.25167 & 0.14667 & 0.44167 & 20000000 & 50334 & 29334 & 88334 \\
\hline & & PEI & $0.467^{1}$ & 0.269 & 0.804 & 400000 & 1868 & 1076 & 3216 \\
\hline & & QC & 3.46667 & 2 & 5.93333 & 20000000 & 693334 & 400000 & 1186660 \\
\hline & & SK & 0.00136 & 0.00079 & 0.00244 & 7000000 & 95 & 55 & 171 \\
\hline & & $\mathrm{YT}$ & 0 & 0 & 0 & 8000000 & 0 & 0 & 0 \\
\hline Total & & & & & & & 819,612 & 472,190 & $1,409,793$ \\
\hline \multirow[t]{14}{*}{ Lark Sparrow } & Vesper & & & & & & & & \\
\hline & Sparrow & $A B$ & 0.00987 & 0.0052 & 0.0164 & 15000 & 1 & 1 & 2 \\
\hline & & $\mathrm{BC}$ & 0 & 0 & 0 & 3000 & 0 & 0 & 0 \\
\hline & & $\mathrm{MB}$ & 0 & 0 & 0 & 6000 & 0 & 0 & 0 \\
\hline & & NB & 0 & 0 & 0 & - & & & \\
\hline & & $\mathrm{NL}$ & & & & - & & & \\
\hline & & NS & 0 & 0 & 0 & - & & & \\
\hline & & NU & & & & - & & & \\
\hline & & NWT & & & & - & & & \\
\hline & & ON & 0.45824 & 0.26294 & 0.81647 & - & & & \\
\hline & & PEI & 0 & 0 & 0 & - & & & \\
\hline & & $\mathrm{QC}$ & 0.33917 & 0.20333 & 0.55833 & - & & & \\
\hline & & SK & 0.03142 & 0.01916 & 0.05294 & 19,000 & 6 & 4 & 10 \\
\hline & & $\mathrm{YT}$ & & & & - & & & \\
\hline Total & & & & & & & 7 & 5 & 12 \\
\hline \multirow{9}{*}{$\begin{array}{l}\text { Black Duck eggs } \\
\text { and nestlings }\end{array}$} & Mallard $^{2}$ & $A B$ & 0.11217 & .0 .05067 & 0.25117 & & & & \\
\hline & $\begin{array}{l}\text { eggs and } \\
\text { nestlings }\end{array}$ & $\mathrm{MB}$ & 0.18090 & 0.12022 & 0.27780 & & & & \\
\hline & & NB & 0.46000 & 0.26000 & 0.76000 & 74,000 & 340 & 192 & 562 \\
\hline & & $\mathrm{NL}^{3}$ & 0.79029 & 0.43553 & 1.3752 & 64,000 & 506 & 278 & 880 \\
\hline & & NS & 1.10000 & 0.60000 & 1.95000 & 74,000 & 814 & 444 & 1443 \\
\hline & & ON & 0.25156 & 0.13938 & 0.47688 & 106,400 & 267 & 148 & 51 \\
\hline & & $\mathrm{PEI}^{3}$ & 0.79029 & 0.43553 & 1.37520 & 26,000 & 205 & 113 & 358 \\
\hline & & QC & 0.81089 & 0.44658 & 1.41560 & 329,000 & 2668 & 1469 & 4657 \\
\hline & & SK & 0.08535 & 0.05230 & 0.13900 & & & & \\
\hline Total & & & & & & & 4801 & 2645 & 7950 \\
\hline \multirow{2}{*}{$\begin{array}{l}\text { Blue-winged teal } \\
\text { eggs and nestlings }\end{array}$} & \multirow{2}{*}{$\begin{array}{l}\text { Mallard } \\
\text { eggs and }\end{array}$} & $A B$ & 0.11217 & .0 .05067 & 0.25117 & 650,000 & 729 & 329 & 1632 \\
\hline & & $\mathrm{MB}$ & 0.18090 & 0.12022 & 0.27780 & 349,500 & 632 & 420 & 971 \\
\hline
\end{tabular}




\begin{tabular}{|c|c|c|c|c|c|c|c|c|c|}
\hline \multirow[b]{2}{*}{ Non-focal Species } & \multirow[b]{2}{*}{$\begin{array}{l}\text { Focal } \\
\text { species }\end{array}$} & \multicolumn{4}{|c|}{ \%Pop focal } & \multirow[b]{2}{*}{$\begin{array}{l}\text { Pop. } \\
\text { Est. }\end{array}$} & \multicolumn{3}{|c|}{$\begin{array}{l}\text { Estimated take (Mortality) } \\
\text { Non-focal species }\end{array}$} \\
\hline & & Region & Median & LB & UB & & Median & LB & UB \\
\hline & nestlings & NB & 0.46000 & 0.26000 & 0.76000 & & & & \\
\hline & & $\mathrm{NL}$ & 0.79029 & 0.43553 & 1.3752 & & & & \\
\hline & & NS & 1.10000 & 0.60000 & 1.95000 & & & & \\
\hline & & ON & 0.25156 & 0.13938 & 0.47688 & 8,200 & 21 & 11 & 39 \\
\hline & & PEI & 0.79029 & 0.43553 & 1.37520 & & & & \\
\hline & & $\mathrm{QC}$ & 0.81089 & 0.44658 & 1.41560 & & & & \\
\hline & & SK & 0.08535 & 0.05230 & 0.13900 & 165,000 & 141 & 89 & 229 \\
\hline Total & & & & & & & 1522 & 847 & 2871 \\
\hline \multirow{9}{*}{$\begin{array}{l}\text { Gadwall eggs and } \\
\text { nestlings }\end{array}$} & \multirow{9}{*}{$\begin{array}{l}\text { Mallard } \\
\text { eggs and } \\
\text { nestlings }\end{array}$} & $A B$ & 0.11217 & .0 .05067 & 0.25117 & 210,000 & 236 & 106 & 527 \\
\hline & & $\mathrm{MB}$ & 0.18090 & 0.12022 & 0.27780 & 105,000 & 190 & 126 & 292 \\
\hline & & NB & 0.46000 & 0.26000 & 0.76000 & & & & \\
\hline & & $\mathrm{NL}$ & 0.79029 & 0.43553 & 1.3752 & & & & \\
\hline & & NS & 1.10000 & 0.60000 & 1.95000 & & & & \\
\hline & & ON & 0.25156 & 0.13938 & 0.47688 & & & & \\
\hline & & PEI & 0.79029 & 0.43553 & 1.37520 & & & & \\
\hline & & $\mathrm{QC}$ & 0.81089 & 0.44658 & 1.41560 & & & & \\
\hline & & SK & 0.08535 & 0.05230 & 0.13900 & 850,000 & 725 & 445 & 1182 \\
\hline Total & & & & & & & 1151 & 677 & 2001 \\
\hline \multirow{9}{*}{$\begin{array}{l}\text { Pintail eggs and } \\
\text { nestlings }\end{array}$} & \multirow{9}{*}{$\begin{array}{l}\text { Mallard } \\
\text { eggs and } \\
\text { nestlings }\end{array}$} & $A B$ & 0.11217 & .0 .05067 & 0.25117 & 250,000 & 280 & 127 & 628 \\
\hline & & $\mathrm{MB}$ & 0.18090 & 0.12022 & 0.27780 & 50,500 & 91 & 61 & 140 \\
\hline & & NB & 0.46000 & 0.26000 & 0.76000 & & & & \\
\hline & & $\mathrm{NL}$ & 0.79029 & 0.43553 & 1.3752 & & & & \\
\hline & & NS & 1.10000 & 0.60000 & 1.95000 & & & & \\
\hline & & ON & 0.25156 & 0.13938 & 0.47688 & & & & \\
\hline & & PEI & 0.79029 & 0.43553 & 1.37520 & & & & \\
\hline & & QC & 0.81089 & 0.44658 & 1.41560 & & & & \\
\hline & & SK & 0.08535 & 0.05230 & 0.13900 & 725,000 & 618 & 279 & 1008 \\
\hline Total & & & & & & & 991 & 567 & 1176 \\
\hline \multirow{9}{*}{$\begin{array}{l}\text { Shoveler eggs and } \\
\text { nestlings }\end{array}$} & \multirow{9}{*}{$\begin{array}{l}\text { Mallard } \\
\text { eggs and } \\
\text { nestlings }\end{array}$} & $A B$ & 0.11217 & .0 .05067 & 0.25117 & 500,000 & 561 & 253 & 1256 \\
\hline & & $\mathrm{MB}$ & 0.18090 & 0.12022 & 0.27780 & 445,000 & 805 & 535 & 1236 \\
\hline & & NB & 0.46000 & 0.26000 & 0.76000 & & & & \\
\hline & & $\mathrm{NL}$ & 0.79029 & 0.43553 & 1.3752 & & & & \\
\hline & & NS & 1.10000 & 0.60000 & 1.95000 & & & & \\
\hline & & ON & 0.25156 & 0.13938 & 0.47688 & & & & \\
\hline & & PEI & 0.79029 & 0.43553 & 1.37520 & & & & \\
\hline & & $\mathrm{QC}$ & 0.81089 & 0.44658 & 1.41560 & & & & \\
\hline & & SK & 0.08535 & 0.05230 & 0.13900 & $1,100,000$ & 939 & 575 & 1529 \\
\hline Total & & & & & & & 2,305 & 1,364 & 4,021 \\
\hline \multirow[t]{5}{*}{ Black Duck adults } & \multirow{5}{*}{$\begin{array}{l}\text { Mallard }^{2} \\
\text { adults }\end{array}$} & $A B$ & 0.00300 & 0.00133 & 0.00683 & & & & \\
\hline & & $\mathrm{MB}$ & 0.00494 & 0.00315 & 0.00741 & & & & \\
\hline & & NB & 0.02000 & 0 & 0.02000 & 74,000 & 15 & 0 & 15 \\
\hline & & $\mathrm{NL}^{3}$ & 0.02248 & 0.01261 & 0.03615 & 64,000 & 14 & 8 & 23 \\
\hline & & NS & 0.02500 & 0.02500 & 0.05000 & 74,000 & 18 & 18 & 37 \\
\hline
\end{tabular}




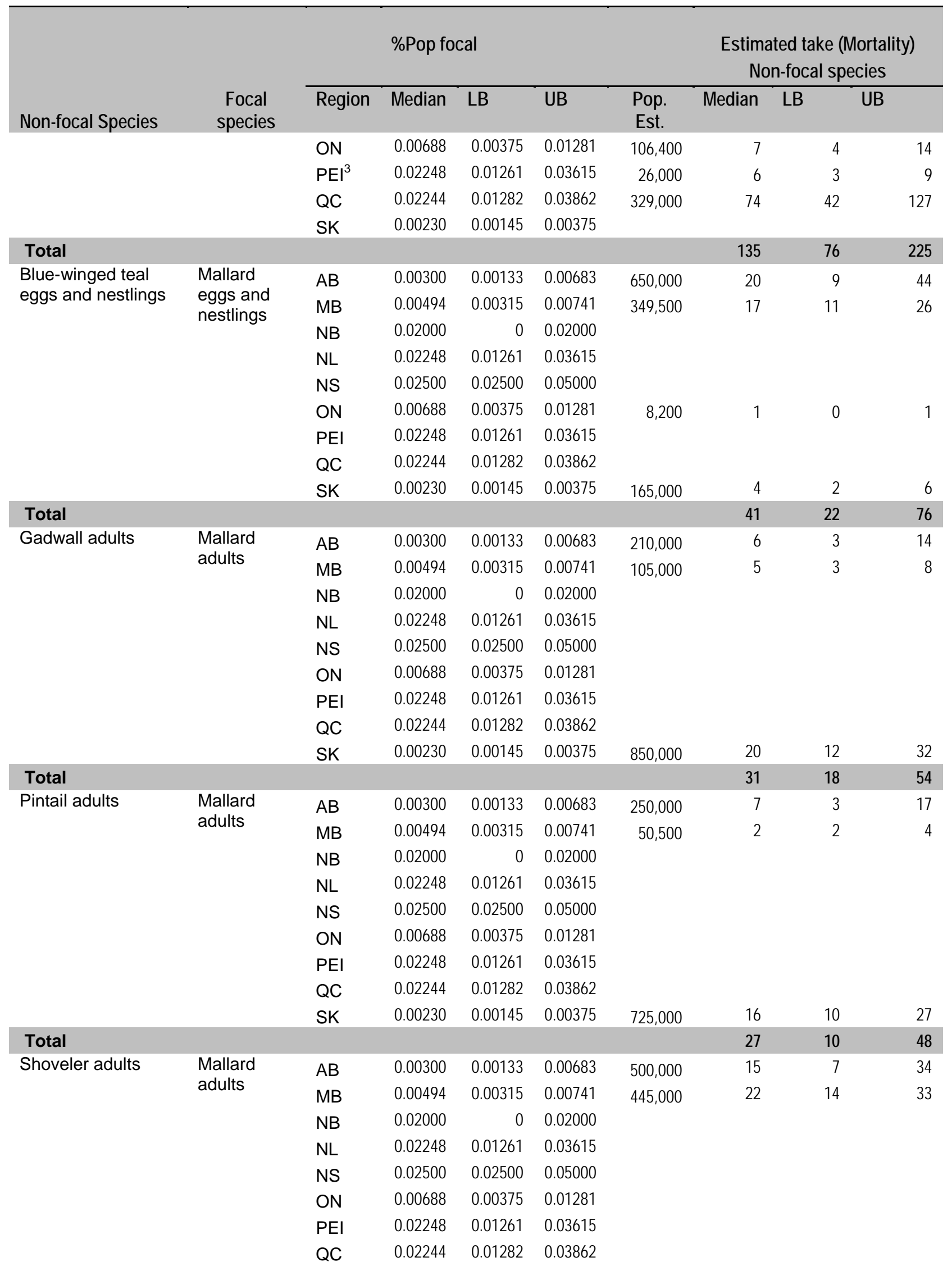




\begin{tabular}{|c|c|c|c|c|c|c|c|c|c|c|}
\hline \multirow[b]{2}{*}{ Non-focal Species } & \multirow[b]{2}{*}{$\begin{array}{l}\text { Focal } \\
\text { species }\end{array}$} & \multicolumn{4}{|c|}{ \%Pop focal } & \multicolumn{5}{|c|}{$\begin{array}{l}\text { Estimated take (Mortality) } \\
\text { Non-focal species }\end{array}$} \\
\hline & & Region & Median & LB & UB & $\begin{array}{l}\text { Pop. } \\
\text { Est. }\end{array}$ & Median & LB & & \\
\hline & & SK & 0.00230 & 0.00145 & 0.00375 & $1,100,000$ & 25 & & 16 & 41 \\
\hline Total & & & & & & & 62 & & 37 & 108 \\
\hline
\end{tabular}

Non-focal Species Totals

$835,730 \quad 480,926 \quad 1,438,354$

$1 \quad$ Clay-colored Sparrow (CCSP) is the focal species for the group containing American Robin (AMRO). AMRO occurs in the maritime provinces, but CCSP does not. To calculate estimated take values for AMRO in the maritime provinces, we averaged CCSP \% take over all provinces/territories in which it occurred, and used these values for AMRO \%Pop focal.

2 Population estimates for all waterfowl (Including Mallards) were not available for BC, or any of the Territories. As no estimates of take were possible those jurisdictions are not included in this table.

3 Because no mallard data were available to calculate density information for Newfoundland and PEI (as the provinces are mostly out of the species' range), we used average results for NB, NS, and QC in this calculation of take for black duck 
Table 11. Total annual estimated incidental take for 18 bird species affected by roadside maintenance operations across Canada. Species indicated in bold are focal species. Estimates are based on median totals in Table 8 and Table 10.

\begin{tabular}{|c|c|c|c|}
\hline Common Name & Estimated Take & Total CDN Pop & $\begin{array}{c}\text { \% Total CDN } \\
\text { Pop }\end{array}$ \\
\hline Killdeer ${ }^{1}$ & 2,026 & $1,613,200$ & 0.1256 \\
\hline Savannah Sparrow & 9,306 & $35,770,000$ & 0.0260 \\
\hline American Goldfinch & 2,833 & $8,410,000$ & 0.0337 \\
\hline Eastern Meadowlark & 129 & 260,300 & 0.0496 \\
\hline Western Meadowlark & 473 & $2,596,000$ & 0.0182 \\
\hline Song Sparrow & 4,175 & $24,030,000$ & 0.0174 \\
\hline Mourning Dove & 1,430 & $5,315,400$ & 0.0269 \\
\hline Indigo Bunting & 181 & 454,000 & 0.0399 \\
\hline Clay-colored Sparrow & 1,088 & $18,982,000$ & 0.0057 \\
\hline American Robin & 819,612 & $139,400,000$ & 0.5880 \\
\hline Vesper Sparrow & 3,053 & $10,692,600$ & 0.0286 \\
\hline Lark Sparrow & 7 & 43,000 & 0.0173 \\
\hline American Black Duck ${ }^{2,3}$ & 4,935 & 673,400 & 0.7328 \\
\hline Blue-winged Teal $^{2,3}$ & 1,563 & $1,172,700$ & 0.1333 \\
\hline Gadwall $^{2,3}$ & 1,182 & $1,165,000$ & 0.1015 \\
\hline Mallard 2,3 & 5,650 & $3,857,800$ & 0.1465 \\
\hline Northern Pintail ${ }^{2,3}$ & 1,017 & $1,025,500$ & 0.0992 \\
\hline Northern Shoveler ${ }^{2,3}$ & 2,367 & $1,757,500$ & 0.1347 \\
\hline $\begin{array}{l}\text { The estimated total Canad } \\
\text { Morrison et al. (2001). }\end{array}$ & size for killdeer is an a & age of 5 values from & icher (2002), includin \\
\hline $\begin{array}{ll}2 & \text { Data shown for waterfowl i } \\
3 & \text { Data for waterfowl populat }\end{array}$ & ggs, nestlings and adu & oyt & \\
\hline
\end{tabular}

Table 12. Total annual estimated take (based on median values from Table 8 and Table 10) for 6 focal species and 12 non-focal species.

\begin{tabular}{|c|c|c|c|c|c|c|c|c|c|c|c|c|c|}
\hline & \multicolumn{13}{|c|}{ Province/Territory } \\
\hline & $A B$ & $B C$ & MB & NB & NL & NS & NWT \&NU & ON & PEI & QC & SK & YT & Total \\
\hline focal species & 3,570 & 238 & 3,452 & 24 & 0 & 88 & 9 & 6,936 & 3 & 3,527 & 7,443 & 8 & 25,298 \\
\hline non-focal spp & 2,970 & 2,765 & 2,653 & 14,365 & 42,550 & 14,850 & 0 & 52,234 & 2,079 & 697,433 & 3,827 & 0 & 835,726 \\
\hline Total & 6,540 & 3,003 & 6,105 & 14,389 & 42,550 & 14,938 & 9 & 59,170 & 2,082 & 700,960 & 11,270 & 8 & 861,024 \\
\hline
\end{tabular}




\section{DISCUSSION}

The biological significance of incidental take for bird populations is as yet unknown. Populations of many species are declining, but the role of take in these declines is anything but clear. The impact of take is likely to vary from species to species, and from region to region. Effects may occur locally but not regionally. For sectors in which incidental take kills individuals regardless of condition (e.g., window strikes at structures), the impact on populations may be greater than for factors that cause mortality among weaker individuals (e.g., predation by house cats). Additionally, incidental take that kills adults can be expected to have a greater impact at the population level than where mortality is predominantly juveniles.

For the roads sector, incidental take is an indiscriminant mortality factor, taking individuals regardless of their condition. On the other hand, take is mostly juvenile birds (eggs and nestlings) rather than adults. We estimated mortality of adult waterfowl, based on documented evidence that they are susceptible to take in agricultural mowing. Our calculations suggest than the total amount of adult take is less than $3 \%$ of that of the take of eggs and nestlings. As such, in general, this source of incidental take affects recruitment but does not greatly diminish the population of breeding adults. Even so, if recruitment gets reduced to the point where it becomes insufficient to maintain the population over the long term, then incidental take will have created a local population sink.

There are no published criteria for what constitutes biologically significant levels of incidental take for bird populations. However, a widely accepted criterion for identifying key habitat sites for population conservation may serve as a suitable surrogate. Key habitat sites are those that are so important that their loss could have a significant detrimental impact on the total population (Latour et al. 2006). Sites believed to support at least $1 \%$ of a Canadian population are considered to be key habitat sites. By extension, losses to incidental take of $1 \%$ or more of a species Canadian population could be considered biologically significant (C. Machtans, pers. com.).

At the national scale, our calculations suggest that take approaches $1 \%$ only for black ducks $(0.73 \%)$. For all other species, levels of take are considerably less than $1 \%$ (Table 11$)$. The seemingly high proportion of take for black ducks seems to be a function of their high population in Quebec, for which incidental take of mallards (the focal species) was estimated to be high. According to the $1 \%$ criterion, incidental take due to roadside maintenance operations is not a biologically significant mortality factor in Canada. In fact, given that most take was for nestlings and eggs, which generally have low survival rates, the impact of this source of take likely very small.

At the provincial scale, clay-colored sparrow was the only focal species that experienced take of $1 \%$ or more of its population. Modelling results showed an estimated $2.0-5.9 \%$ (median 3.5\%) of the Quebec population clay-colored sparrows is killed each year by roadside maintenance operations. Two factors contributed to this result. Quebec represents the easternmost limit of clay-colored sparrow breeding range, and the provincial population is small relative to populations in all other provinces and territories in which it occurs. The number of birds lost to incidental take will have a larger proportional impact on small populations than on larger ones, resulting in a relatively high value for Quebec. Additionally, the species range in Quebec covers the road-rich southwestern corner of the province, where over $13,000 \mathrm{~km}$ of road intersect with clay-colored sparrow breeding range (see Table 7 ). If the $1 \%$ criterion can also be applied at the provincial scale, then it is likely that maintenance-related incidental take has a biologically 
significant impact on the clay-colored sparrow population in Quebec. No other focal species experienced take of $1 \%$ or more of their provincial population.

Among the non-focal species, the American robin in Quebec experiences the highest total incidental take due to roadside maintenance. Our model estimated that 820,000 eggs and nestlings are destroyed annually in Quebec as a result of roadside maintenance operations. This result is largely an artifact of the way take has been calculated. The focal species for American robin was clay-colored sparrow which experienced particularly high proportional take in Quebec. Applying this high proportional take to the population estimate for robins in Quebec $(20,000,000$ birds) results in a very large number of birds lost to incidental take. Consequently, Quebec accounts for the bulk (84.6\%) of all incidental take of American robins in Canada, and American robin take comprises $95 \%$ of the total take for Canada. Even so, this level of take represents only $0.588 \%$ of the Canadian population of American robin (139,400,000 birds), so does not exceed the $1 \%$ criterion for biological significance.

Maintained road rights-of-way are open habitats. Many of the birds that nest along roadsides are grassland birds. As a group, grassland birds have experienced population declines over the past three decades that far exceed those of any other group of North American birds; loss of grassland habitat on breeding grounds is the most likely cause (Herkert et al. 2003). In areas of intensive agriculture, roadsides may represent an attractive alternative habitat for many open country bird species (Oetting and Cassel 1971; Voorhees and Cassel 1980; Dale 1993; Belanger et al. 2006). On the other hand, the benefits to productivity of this alternative habitat are counter-balanced at least somewhat by the risks associated with it. Not only is there a demonstrable risk of mortality from maintenance operations in roadside habitats, there is also higher mortality from predation in these habitats (Haensly et al. 1987; Camp and Best 1994). Road rights-of-way are linear, and can serve as travel corridors for mammalian predators (Dale 1993). Additionally, productivity can be adversely affected by avian predators, particularly if the right-of-way contains a fenceline (Evans and Wolfe 1967; Meunier et al. 2000). Brood parasitism by the brown-headed cowbird can also be a factor that affects productivity in habitats with a lot of edge, such as a roadside (Herkert et al. 2003).

Despite the risks associated with roadside nesting, there is still tremendous potential for managing roadside habitats to offset productivity losses among grassland and other open country bird species in Canada. Where roadside mowing can be avoided, or delayed until after young-of-the-year have fledged, nesting success will improve (Oetting and Cassel 1971; Berner 1984:32 in MDNR 2005; Dale 1993; Cook and Daggett 1995; Leif 2004).

\section{Mitigating Incidental Take}

Some provinces have environmental policies in place that prohibit the incidental take of birds (nests, eggs, nestlings) during road maintenance and construction operations. In British Columbia, maintenance specifications state that the contractors must comply with the Province's Environmental Best Practices for Highway Maintenance Activities (BCMTI 2009a: Section 5.10). These standards state that operations must cause "No injury, molestation or destruction of a bird, its eggs, and occupied nest, or the nest of an eagle, Peregrine Falcon, Gyrfalcon, Osprey, heron, or Burrowing Owl, unless the species is listed under Schedule C as exempt from this protection (Wildlife Act, Section 34)". Permits must be obtained for any activity that will affect migratory birds. Construction work must comply with federal (including the MBCA), provincial, municipal and local laws to ensure that work does not adversely affect the environment (BCMTI 2009b, Section 165). If clearing must occur during the breeding season for birds, the area is surveyed for active nests before work begins. If active nests are found, clearing is either delayed or a $30 \mathrm{~m}$ "no clear" buffer is established to mitigate disturbance 
(Angela Buckingham, pers. com.). These requirements are expressly stated in construction contracts.

In Ontario, specific environmental protection requirements are imposed on road maintenance and construction operations by the Ministry of Transportation Ontario (MTO), including the protection of active bird nests (MTO 2006a:11; MTO 2006b:12). Construction contracts contain "Bird Nesting Preventative Measures" requirements (NSP 9051) that state "No work is permitted to proceed that would result in the destruction of active nests (nests with eggs or young birds), or the wounding or killing of birds, of species protected under the Migratory Birds Convention Act, 1994 and/or Regulations under that Act". Maintenance operations must comply with Maintenance Special Provision SSP 080S06, which requires operators to conduct a visual inspection for bird nests in all areas of work. If active nests are found, operators must either protect the nest or suspend operations. MTO takes the migratory bird issue "very seriously", and the agency is continually looking for better ways to minimize interference with active nesting (John Small, pers. com.). Because these mitigations are not strictly timing mitigations, i.e., operations occur during the breeding season, our modeling results will probably overestimate incidental take for Ontario's provincially-managed roads by reporting take that may not actually occur.

The Province of Saskatchewan requires that road construction operations comply "with all federal, provincial, municipal and local laws and regulations which seek to ensure that construction work does not adversely affect the environment", including the Migratory Bird Convention Act (see http://www.highways.gov.sk.ca/1650/). Timing is not specified in construction contracts, but provisions are included to allow the imposition of timing restrictions and set-backs in the event that a "sensitive species" is encountered on the job site (Nichole Andre, pers. com.). Saskatchewan does not appear to similarly regulate roadside maintenance operations.

In Newfoundland and Labrador, the Environment Assessment process constrains the timing of land clearing for road construction such that it minimizes the disturbance/destruction of bird nests. For example, a recent screening report states that the Department of Transportation and Works (DTW) will conduct a pre-construction survey for migratory birds and nesting locations; nests observed within the work area will be flagged and the vegetation within the immediate surrounding area will remain undisturbed during construction activities (Transport Canada 2009). As a general rule, DTW tries to avoid clearing between April and August (John Morrissey, pers. com.). Roadsides in the province are not mowed.

British Columbia, Ontario, Saskatchewan and Newfoundland/Labrador are the only provinces that offered information about their policies and best practices that mitigate against losses of bird nests during maintenance and construction operations. Other provinces/territories may also follow such procedures, but more research is needed to explore this possibility.

\section{Caveats and Assumptions}

There are several caveats which should be taken into account in interpreting this analysis:

- obviously, the approach used is susceptible to anomalous circumstances (such as which exist for clay-coloured sparrow, and American robin);

- the density data used to calibrate the models (from the CBBC) are sparse for many species and habitats, and so may not be accurate in all circumstances;

- waterfowl data were unavailable for British Columbia, and the territories and so estimates of take for ducks are underestimated; 
- there are almost certainly species other than those modelled in this paper which nest in roadside habitat, and which are therefore likely susceptible to incidental take. The total estimate of incidental take estimated for this project is likely an underestimate (notwithstanding the odd result for American robin);

- there are several elements of this approach which called for educated judgments to be used (i.e. lumping of habitat types, estimates of the relative value of roadside habitats), and adding further uncertainty to the results.

\section{Next Steps}

The results generated here should be viewed as very rough estimates of incidental take. Many assumptions were required for the modeling exercise. Estimates could be improved by:

- deriving better bird density estimates for each province/territory, including incorporation of data from the Northwest Territories / Nunavut Bird Checklist Survey and data for Prince Edward Island

- modelling each of the roadside nesting species in the list separately to improve the integrity of the incidental take estimates; applying the percent take from focal species to non-focal species within the same functional group implicitly assumes that the proportion of the population in roadside vs. non-roadside habitat is the same for both populations; this may be a particularly poor assumption for non-focal species with very large populations (e.g., American robin); in this case, the total available roadside habitat may be too small to realistically hold the same proportion of the total population

- validate/revise habitat quality multipliers with research and expert opinion

- more research into the birds that might be vulnerable to roadside maintenance in the taiga/tundra (gulls/terns, shorebirds, waterfowl)

- more research into the kinds of road maintenance that occur, if any, in Nunavut

- addition of "Arctic" habitat type to represent tundra (it is currently represented as needed in the Grassland, Shrubland and Wetland habitat types)

- more research into accounting for latitudinal differences in initiation dates, clutch size, incubation time, nestling period, fledging dates and nesting density

- research inter-annual variation in nest initiation dates to bound the simulated dates

- incorporate right-of-way width into estimating nest densities (e.g., wide rights-of-way are more likely to support waterfowl)

- more research into maintenance on permanent resource roads; take from this type of road is probably under-represented in our estimates because, at least in some provinces (e.g., Ontario), resource roads are managed by the natural resources ministry rather than by transportation; information about maintenance activities conducted by these agencies needs to be obtained

- gather information from Parks Canada about its maintenance activities in national parks

- improve estimates of area disturbed by maintenance operations by 1) obtaining information from non-respondent provinces and Nunavut, and 2) incorporating information from provinces that responded too late for their input to be used in the analysis and reporting (e.g., Ontario and Saskatchewan municipalities) 


\section{REFERENCES}

Alberta NAWMP Partnership Management Committee. 2008. Alberta NAWMP Implementation Plan 2007-2012. Prairie Habitat Joint Venture. 56 p.

Ankney, C.D. 1985. Habitat selection by roof-nesting killdeer. Journal of Field Ornithology 56(3):284-286

Best, L.B., K.E. Freemark, J.J. Dinsmore and M. Camp. 1995. A Review and Synthesis of Habitat Use by Breeding Birds in Agricultural Landscapes of lowa. American Midland Naturalist 134(1):1-29

Blancher, P.J. 2002. Importance of breeding birds exported from Canada - Information on landbirds and shorebirds. Environment Canada. 46 pp + appendices.

Blancher, P. J., K. V. Rosenberg, A. O. Panjabi, B. Altman, J. Bart, C. J. Beardmore, G. S. Butcher, D. Demarest, R. Dettmers, E. H. Dunn, W. Easton, W. C. Hunter, E. E. Iñigo-Elias, D. N. Pashley, C. J. Ralph, T. D. Rich, C. M. Rustay, J. M. Ruth, and T. C. Will. 2007. Guide to the Partners in Flight Population Estimates Database. Version: North American Landbird Conservation Plan 2004. Partners in Flight Technical Series No 5.

British Columbia Ministry of Transportation and Infrastructure (BCMTI). 2009a. Environmental Best Practices for Highway Maintenance Activities, January 2009. Edited by Allan D. Planiden and Leonard E. Sielecki. 154 pp.

British Columbia Ministry of Transportation and Infrastructure (BCMTI). 2009b. 2009 Standard Specifications for Highway Construction. Adopted October 1, 2008. Volume 1 of 2. 586 pp.

Casella, G. and R.L. Berger. 2002. Statistical Inference, $2^{\text {nd }}$ Edition. Duxbury, Pacific Grove California, USA.

Catlin, D.H. and D.K. Rosenberg. 2006. Nest destruction associated with mortality and dispersal of Burrowing Owls in the Imperial Valley, California. The Southwestern Naturalist 51(3):406-409

Camp, M. and L.B. Best. 1994. Nest Density and Nesting Success of Birds in Roadsides Adjacent to Rowcrop Fields. American Midland Naturalist 131(2):347-358

Cook, K.E. and P-M. Daggett. 1995. Highway Roadkill, Safety, and Associated Issues of Safety and Impact on Highway Ecotones. Unreviewed report prepared for the Task Force on Natural Resources (A1F52), Transportation Research Board, National Research Council.

Dale, B. 1993. Effects of haying on waterfowl, upland game birds and shorebirds. In Proceedings of the Third Prairie Conservation and Endangered Species Workshop, February 1992. Provincial Museum of Alberta Natural History Occasional Paper No. 19:22-26.

Dale, B.C., P.A. Martin, P.S. Taylor. 1997. Effects of Hay Management on Grassland Songbirds in Saskatchewan. Wildlife Society Bulletin 25(3):616-626 
Evans, R.D. and C.W. Wolfe. Jr. 1967. Waterfowl production in the rainwater basin area of Nebraska. Wildl. Manage. 31:788-794

Latour, P.B., J. Leger, J.E. Hines, M.L. Mallory, D.L. Mulders, H.G. Gilchrist, P.A. Smith and D.L. Dickson. 2006. Key migratory bird terrestrial habitat sites in the Northwest Territories and Nunavut. Third Edition. Canadian Wildlife Service Occasional Paper, March 2006. Partial copy available online at:

http://www.ngps.nt.ca/Upload/Interveners/Environment\%20Canada/key terrestrial part1 mar06 .pdf. Accessed April 22, 2010.

Laursen, K. 1981. Birds on roadside verges and the effect of mowing on frequency and distribution. Biological Conservation 20:59-68

Leif, A. P. 2004. Avian nest densities and success in state highway roadsides in South Dakota. Completion Report 2004-11, South Dakota Department of Game, Fish and Parks, Pierre.

Efron, B. and R.J. Tibshirani. 1994. An Introduction to the Bootstrap. Monographs on Statstics and Applied Probability 57. Chapman \& Hall/CRC. Boca Raton, FL.

Farris, A.L., E.D. Klonglan and R.C. Nomsen. 1977. The Ring-necked Pheasant in lowa. lowa Conservation Commission, Des Moines.

Forman, R.T., D. Sperling, J.A. Bissonette, A.P. Clevenger, C.D. Cutshall, V.H. Dale, L. Fahrig, R. France, C.R. Goldman, K. Heanue, J.A. Jones, F.J. Swanson, T. Turrentine and T.C. Winter. 2003. Road Ecology Science and Solutions. Island Press, Washington. 481 pp.

Haensly, T.F., J.A. Crawford and S.M. Myers. 1987. Relationships of habitat structure to nest success of Ring-necked Pheasants. J. Wildl. Manage. $51: 421-425$

Hergenrader, G.L. 1962. The Incidence of Nest Parasitism by the Brown-Headed Cowbird (Molothrus ater) on Roadside Nesting Birds in Nebraska. The Auk 79(1):85-88

Herkert, J.R., D.L. Reinking, D.A. Wiedenfeld, M. Winter, J.L. Zimmerman, W.E. Jensen, E.J. Finck, R.R. Koford, D.H. Wolfe, S.K. Sherrod, M.A. Jenkins, J. Faaborg and S.K. Robinson. 2003. Effects of Prairie Fragmentation on the Nest Success of Breeding Birds in the Midcontinental United States. Conservation Biology 17(2):587-594

Hilborn, R. and M. Mangel. 1997. The ecological detective: confronting models with data. Mongr. Pop. Biol. No. 28. Princeton University Press, Princeton, N.J.

Jackson, B.J. and J.A. Jackson. 2000. Killdeer (Charadrius vociferus), The Birds of North America Online (A. Poole, Ed.). Ithaca: Cornell Lab of Ornithology; Retrieved from the Birds of North America Online: http://bna.birds.cornell.edu/bna.html/species/517

Jacobson, S.L. 2005. Mitigation Measures for Highway-caused Impacts to Birds. USDA Forest Service Gen. Tech. Rep. PSW-GTR-191. pp. 1043-1050

Joselyn. G.B.. J.E. Warnock. and S.L. Etter. 1968. Manipulation of Roadside Cover for Nesting Pheasants--a Preliminary Report. J. Wildl. Manage. 32(2):217-233 
Kennedy, J, P. Dilworth-Christie and Erskine. 1999. The Canadian Breeding Bird (Mapping) Census Database. Technical Report Series No. 342, Canadian Wildlife Service, Ottawa, Ontario. Available online at: http://dsp-psd.pwgsc.gc.ca/Collection/CW69-5-342E.pdf.

Maguire, C.C. 2007. Oregon Strategies for Transportation Compliance with the Migratory Bird Treaty Act. UC Davis: Road Ecology Center. Retrieved from: http://escholarship.org/uc/item/0sw2c380

Manitoba Implementation Plan Committee. 2008. Manitoba NAWMP Implementation Plan: 2007-2012. Prairie Habitat Joint Venture. 49 p. + appendices.

McGraw, Kevin J. and Alex L. Middleton. 2009. American Goldfinch (Carduelis tristis), The Birds of North America Online (A. Poole, Ed.). Ithaca: Cornell Lab of Ornithology; Retrieved from the Birds of North America Online: http://bna.birds.cornell.edu/bna/species/080

Ministry of Transportation Ontario (MTO). 2006a. Environmental Protection Requirements for Transportation Planning and Highway Design, Construction, Operation and Maintenance, October 2006. Ministry of Transportation Ontario.

Ministry of Transportation Ontario (MTO). 2006b. Environmental Reference for Highway Design, Section 3.2: Terrestrial Ecosystems, October 2006. Ministry of Transportation Ontario.

Minnesota Department of Natural Resources (MDNR). 2005. Roadside Wildlife Habitat Legislative Report. Report prepared in consultation with Minnesota Department of Transportation. $37 \mathrm{pp}$. Available online at: http://archive.leg.state.mn.us/docs/2005/mandated/050422.pdf

Meunier, F.D., C. Verheyden and P. Jouventin. 2000. User of roadsides by diurnal raptors in agricultural landscapes. Biological Conservation 92: 291-298

Morrison, R.I.G., R.E. Gill, Jr., B.A. Harrington, S. Skagen, G.W. Page, C.L. Gratto-Trevor and S.M. Haig. 2001. Estimates of shorebird populations in North America. Occasional Paper No. 104, Canadian Wildlife Service, Ottawa, Ontario. 64 pp.

Oetting. R.B. and J.F. Cassel. 1971. Waterfowl Nesting on Interstate Highway Right-of-Way in North Dakota. J. Wildl. Manage. 35(4) :774-781

Peck, G. and R. James. 1983. Breeding birds of Ontario Nidiology and distribution. Volume 1 - Non-passerines. A Life Science Miscellaneous Publication of the Royal Ontario Museum, Toronto. $321 \mathrm{pp}$.

Peck, G. and R. James. 1987. Breeding birds of Ontario Nidiology and distribution. Volume 2 - Passerines. A Life Science Miscellaneous Publication of the Royal Ontario Museum, Toronto. 387 pp.

Payne, Robert B. 2006. Indigo Bunting (Passerina cyanea), The Birds of North America Online (A. Poole, Ed.). Ithaca: Cornell Lab of Ornithology; Retrieved from the Birds of North America Online: http://bna.birds.cornell.edu/bna/species/004

Powell, A.N. 1993. Brood rearing and chick behavior in killdeer Charadrius vociferus and piping plovers C. melodus. Wader Study Group Bulletin 71:15-16 
Saskatchewan NAWMP Technical Committee. 2008. Saskatchewan NAWMP Implementation Plan 2001-2026. Five Year Plan April 2007 - April 2012. Prairie Habitat Joint Venture. 73 p.

Shochat, E., D.H. Wolfe, M.A. Patten and D.L. Reinking and S.K. Sherrod. 2005. Tallgrass prairie management and bird nest success along roadsides. Biological Conservation 121:399407

Spellerberg, I.F. 1998. Ecological Effects of Roads and Traffic: A Literature Review. Global Ecology and Biogeography Letters 7(5):317-333

Tews, J., P. Mineau and D.G. Bert. 2009. Avian mortality related to mowing and other mechanical operations in agricultural landscapes. Draft report prepared for Environment Canada, Science and Technology Branch, Ottawa. 41 pp.

Trombulak, S.C. and C.A. Frissell. 2000. Review of Ecological Effects of Roads on Terrestrial and Aquatic Communities. Conservation Biology 14(1):18-19

Voorhees, L.D. and J.F. Cassel. 1980. Highway right-of-way: Mowing versus succession as Related to Duck Nesting. J. Wildl. Manage. 44(1):155-163.

Warner, R.E. 1992. Nest ecology of grassland passerines on road rights-of-way in central Illinois. Biological Conservation 59:1-7

Wheelwright, N. T. and J. D. Rising. 2008. Savannah Sparrow (Passerculus sandwichensis), The Birds of North America Online (A. Poole, Ed.). Ithaca: Cornell Lab of Ornithology; Retrieved from the Birds of North America Online: http://bna.birds.cornell.edu/bna/species/045 


\section{APPENDIX A: CONTACTS}

A total of 32 people were contacted for information during the course of the project, most multiple times.

Contacts were asked for the following information on a provincial/territorial scale:

- what types of roadside maintenance occur in your province/territory?

- how much area (in hectares) is affected by each type of maintenance?

- when does each type of maintenance occur?

The collection of roadside disturbance information began on January $4^{\text {th }}, 2010$, and attempts to obtain good quality data continued for a full month. Early in the process of collecting these data, it became clear that many jurisdictions did not have ready access to the information we needed. In some cases (e.g., Saskatchewan, Ontario), many kilometers of road are maintained by individual Municipalities and maintenance information is simply not available at a provincial scale; getting it would require contacting many hundreds of people (e.g., there are 444 Municipalities in Ontario alone). For Ontario, the Ontario Good Roads Association agreed to circulate a simple survey to members to collect the information we needed directly from the municipalities that do the maintenance. At the time of writing, 33 responses had been received. Our contact at the Saskatchewan Association of Rural Municipalities also circulated a survey to members, and 18 responses were received. British Columbia also has a tiered road maintenance system, and information from the Districts about their maintenance activities is only available from the individual Districts. In this case, however, the proportion of roads under District management is very small relative to that managed by the Province (Angela

Buckingham, pers. com.).

\section{Roadside Disturbance Inquiries}

Thirty-two people were contacted across the country, some multiple times, for information about the area of roadside maintained and the area of land cleared for road construction, and the timing of these works. The following table lists those people whose contributions moved our research forward, and summarizes their input.

British Columbia

Dianne Froese, Manager Procurement, Highway Maintenance Contracts Dianne.Froese@gov.bc.ca

Fred Hughes, District Operations

Manager - West Kootenay District

Fred.C.Hughes@gov.bc.ca

Brent Bailey

West Kootenay District

250-354-6517

Monique Meek

Monique.Meek@gov.bc.ca

Angela Buckingham, Chief

Environmental Officer, Ministry of

Transportation and Infrastructure roadside maintenance is performed by independent contractors who are paid a fixed price per month, and her office doesn't track their activities; maintenance is done according to general specs, but these don't dictate what time of the year or how much to mow/brush; the Districts oversee maintenance; maintenance operations must comply with all applicable federal, provincial and local laws, including the MBCA; if maintenance is going to impact birds and/or nests, a permit can be obtained from WLAP

forwarded our request to Brent Bailey for response (see next entry)

there is no province-wide summary of maintenance operations on roads managed by the Districts; data for provincial roads should be available in an annual summary road maintenance report; contact Monique Meek in Victoria forwarded our request to Angela Buckingham

agreed to collect the required data (both maintenance and construction); not readily available so will take some time; can only provide information for roads under Provincial management, but these constitute the vast majority of roads in $\mathrm{BC}$; timing windows for maintenance and 


\begin{tabular}{|c|c|}
\hline Angela.Buckingham@gov.bc.ca & $\begin{array}{l}\text { construction, during which operations are prohibited in order to protect nests/eggs/nestlings, } \\
\text { vary depending on latitude; to date, have not received land clearing for construction information } \\
\text { for BC }\end{array}$ \\
\hline $\begin{array}{l}\text { Marni Fedoruk, Project Analyst } \\
\text { Environment, BC Ministry of } \\
\text { Transportation and Infrastructure } \\
\text { Marni.Fedoruk@gov.bc.ca } \\
\end{array}$ & 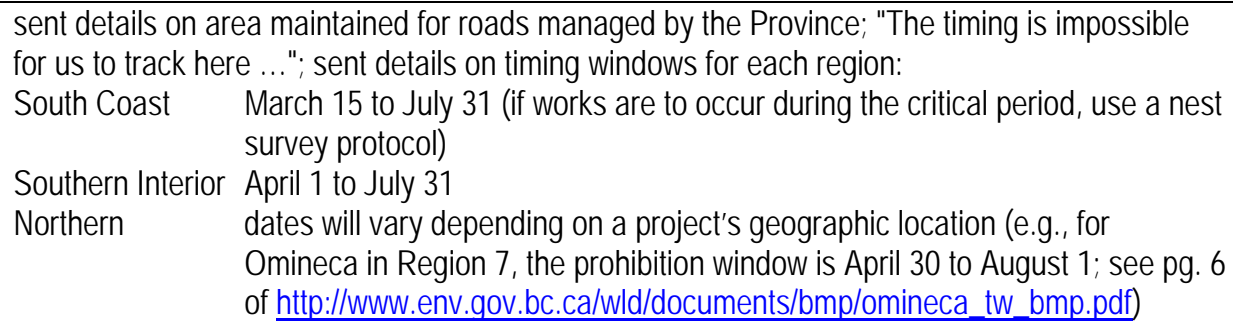 \\
\hline
\end{tabular}

Alberta

Don Collins, Divisional Coordinator, Alberta Transportation (don.collins@gov.ab.ca

mowing is typically done once or twice per year on high volume highways (i.e., divided highways and busy undivided ones); mowing is done late in the season when the grasses are high; less busy roads (including unpaved ones) are typically not mowed at all; 2 additional contacts have elicited no further response

\section{Saskatchewan}

Nichole Andre, Preservation Standards Engineer, Saskatoon Ministry of Highways \& Infrastructure nichole.andre@gov.sk.ca)

\section{Dale Harvey, Assistant Executive}

Director, Saskatchewan Association of Rural Municipalities dharvey@sarm.ca) the Province manages $>26,000 \mathrm{~km}$ of provincial highways; the rural municipal road system, managed by local governments throughout the province, covers nearly an additional 134,000 $\mathrm{km}$ (mostly gravel roads); sent requested information for both maintenance and construction; suggested contacting the Saskatchewan Association of Rural Municipalities for information about rural roads information on area maintained and area cleared for construction is not tracked; gave only a general sense of timing; indicated concern about asking SARM members for information about their maintenance activities because municipalities have been frustrated lately by "illogical and unnecessary cost increases and delays due to regulations and processes of Fisheries and Oceans and Navigable Waters."

\section{Manitoba}

\section{Kimber Osiowy, Manager of} Environmental Services, Manitoba Infrastructure and Transportation Ph: 204 945-2053

David Block, Manitoba Infrastructure and Transportation david.block@gov.mb.ca forwarded our request to David Block (see next entry)

explained our data requirements on the phone and with a follow-up email (including introductory letter from EC); 2 additional contacts (by email) have not yielded any information from Manitoba

\section{Ontario}

John Small, Environmental Planner, Ontario Ministry of Transportation john.small@ontario.ca

Ontario Good Roads Association Scott Butler, Policy and Research Manager

Ph: $905-795-2555 \times 24$

Ontario Good Roads Association

Frank Hull, Manager of Technical Services
Provincial Roads: standards for conducting mowing and vegetation control activities are generally based on the height of the vegetation in relation to visibility/traffic safety, but there is a general Special Provision (SSP 080S06) which applies to all such operations and imposes specific requirements for the monitoring/reporting of bird nesting activity; "MTO takes the migratory bird issue very seriously, and we are always looking for better ways to minimize our interference with active nesting. This week, for example, I was involved in a meeting with a falconer, whose services we are considering to deter swallows from nesting on structures slated for construction activity which would otherwise destroy their nests." recommended that we speak to Frank Hull

no central database of information about road right-of-way maintenance, or of area cleared for road construction; municipalities handle these operations on their own; gave us a contact for Wellington County as a starting point, and offered to provide more names if we decided to do a 


\begin{tabular}{|l|l|}
\hline frank@ogra.org & sample of the municipalities \\
\hline $\begin{array}{l}\text { Paul Johnson, Operations Manager } \\
\text { Wellington County } \\
\text { paulj@wellington.ca }\end{array}$ & $\begin{array}{l}\text { offered to circulate a simple survey to OGRA members; did this immediately, and received 20 } \\
\text { responses within the first 2 weeks (there are 444 municipalities in Ontario that manage 145,000 } \\
\text { km of highway) }\end{array}$ \\
\hline
\end{tabular}

\section{Quebec}

Yves Bedard

Transportation Quebec, Quebec

ybedard@mtq.gouv.qc.ca this person is one of the authors of the Quebec mowing report (Belanger et al. 2006); called (left message) and sent follow-up email detailing our data requirements; received 1 emailed response "I received your request for information and I hope to answer it as soon as possible and in best my knowledge but that will take some time."; followed up again by email, but to date we have received no information for Quebec
New Brunswick

\begin{tabular}{|c|c|}
\hline $\begin{array}{l}\text { Kevin Maclean, Assistant Director } \\
\text { Highway Maintenance and } \\
\text { Environment, Transportation } \\
\text { Kevin.MACLEAN@gnb.ca } \\
\end{array}$ & $\begin{array}{l}\text { forwarded our request for construction information to Dale Forster, and will try to compile the } \\
\text { information we need on maintenance by the middle of next week; to date, we have received no } \\
\text { maintenance information for New Brunswick }\end{array}$ \\
\hline $\begin{array}{l}\text { Dale A. Forster, Director of } \\
\text { Construction, New Brunswick } \\
\text { Department of Transportation } \\
\text { Ph (506) 453-2673, email: } \\
\text { dale.forster@gnb.ca }\end{array}$ & to date, we have received no construction information for New Brunswick \\
\hline \multicolumn{2}{|l|}{ Nova Scotia } \\
\hline $\begin{array}{l}\text { Charles MacDonald, Executive Director } \\
\text { Maintenance and Operations } \\
\text { Department of Transportation and } \\
\text { Infrastructure Renewal } \\
\text { macdonch@gov.ns.ca }\end{array}$ & $\begin{array}{l}\text { called to say he would collect the information we need, and we sent email with introductory } \\
\text { letter; called to follow up and left message; to date, no information has been received for Nova } \\
\text { Scotia }\end{array}$ \\
\hline
\end{tabular}

\section{Prince Edward Island}

Robert MacKinnon, Inventory Control

Manager, Highway Maintenance,

Transportation and Infrastructure

ramackinnon@gov.pe.ca

Stephen Yeo, Chief Engineer/Director, Capital Projects, Transportation and

Infrastructure

$\mathrm{Ph}$ (902) 368-5105

sjyeo@gov.pe.ca received required maintenance information, including pictures of the equipment used for mowing and brushing; was directed to Stephen Yeo (Chief Engineer/Director, Capital Projects) for land clearing for construction information

called and left message; followed up with detailed email and introductory letter; to date, no construction information has been received for PEI

\section{Newfoundland/Labrador}

John Morrissey

Transportation and Works

morrissevi@gov.nl.ca roadsides are not mowed; brushing occurs late in the season (late October through December); clearing for road construction also occurs late October through December, except in Labrador where construction seasons is short; received all required information

\section{Nunavut}

John Hawkins, Director of Transportation Policy \& Planning

Division

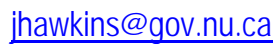

called and left a message; followed up with detailed email and introductory letter; called again a week later and left another message; to date, no information has been received for Nunavut

Northwest Territories

Kevin McLeod, Director Highways \&

Marine forwarded our request to Adnan Aamir (see next entry) 


\begin{tabular}{|l|l|}
\hline KEVIN MCLEOD@gov.nt.ca & \\
\hline Adnan Aamir, Assistant Director & received all required information \\
Highways Operations, Department of & \\
Transportation & \\
Adnan Aamir@gov.nt.ca & \\
\hline
\end{tabular}

Yukon Territory

\begin{tabular}{l|l|}
$\begin{array}{l}\text { Don Hobbis, Director Transportation } \\
\text { Maintenance Branch } \\
\text { don.hobbis@gov.yk.ca }\end{array}$ & $\begin{array}{l}\text { received all required maintenance information from Mr. Hobbis' administrative assistant, Sonia } \\
\text { Gay (sonia.gay@gov.yk.ca) }\end{array}$ \\
\hline $\begin{array}{l}\text { Robin Walsh, Director Transportation } \\
\text { Engineering } \\
\text { robin.walsh@gov.yk.ca }\end{array}$ & received all required construction information \\
\hline
\end{tabular}

\section{General Inquiries}

A total of 18 people were contacted for information about what studies have already been done on avian mortality as a result of roadside mowing/brushing. The following table lists those people whose contributions moved our research forward, and summarizes their input.

\begin{tabular}{|c|c|}
\hline $\begin{array}{l}\text { Angela Kociolek, Research Scientist } \\
\text { Road Ecology Program Area, Western } \\
\text { Transportation Institute } \\
\text { Montana State University } \\
\text { angela.kociolek@coe.montana.edu }\end{array}$ & $\begin{array}{l}\text { identified Catlin and Rosenberg (2006) as the only attempt she knows to quantify incidental take } \\
\text { due to roadside maintenance operations }\end{array}$ \\
\hline $\begin{array}{l}\text { Mandy Karch, Co-ordinator } \\
\text { Ontario Road Ecology Group } \\
\text { mkarch@torontozoo.ca }\end{array}$ & $\begin{array}{l}\text { "... the lack of data points to the importance of this type of research"; OREG only has a small } \\
\text { set of vehicle strike data }\end{array}$ \\
\hline $\begin{array}{l}\text { Chris C. Maguire } \\
\text { Geo-Environmental Section, Oregon } \\
\text { Department of Transportation } \\
\text { christine.c.maguire@ODOT.state.or.us } \\
\end{array}$ & $\begin{array}{l}\text { "... ODOT has no data on bird mortality in the right-of-way. We know it exists, but no one has } \\
\text { any idea about the magnitude." }\end{array}$ \\
\hline $\begin{array}{l}\text { Carmelita Nelson, Coordinator } \\
\text { Roadsides for Wildlife (Minnesota) } \\
\text { Carmelita.nelson@dnr.state.mn.us } \\
\end{array}$ & $\begin{array}{l}\text { the program provides local road authorities with information on state mowing laws with the aim } \\
\text { of reducing disturbance of nesting wildlife } \\
\text { "There is some research, but not enough"; sent excerpt from a book on ring-necked pheasant } \\
\text { (Farris et al. 1977) that includes some roadside density information and a recommendation to } \\
\text { not mow, but no data or references for mowing-related mortality; sent excerpt (literature review) } \\
\text { from MDNR (2005) (written by Ken Varland)that contains nest success/density information for } \\
\text { pheasant, partridge, grouse and waterfowl, but no data or references for mowing-related } \\
\text { mortality }\end{array}$ \\
\hline $\begin{array}{l}\text { Keith Hobson, Research Scientist } \\
\text { Environment Canada, Saskatoon } \\
\text { Ph: 306-975-4102 }\end{array}$ & $\begin{array}{l}\text { author of incidental take report for the forestry sector; called and left message asking if he had } \\
\text { calculated species-specific take results and, if so, would he be willing to share them for use in } \\
\text { the land clearing for road construction portion of the roads tally; to date, no response has been } \\
\text { received }\end{array}$ \\
\hline $\begin{array}{l}\text { Pierre Mineau, Head } \\
\text { EC-Ecopathology, Ottawa } \\
\text { Pierre.Mineau@ec.gc.ca }\end{array}$ & $\begin{array}{l}\text { shared background information for Tews et al. (2009) incidental take report for the agriculture } \\
\text { sector (related papers, breeding and population data, including regression equations used); had } \\
\text { no data or papers on avian mortality due to roadside mowing; suggested I contact Luc Belanger } \\
\text { to ask about his recent study of mowing impacts on roadside habitat quality in southern Quebec }\end{array}$ \\
\hline $\begin{array}{l}\text { Luc Belanger, Manager } \\
\text { CWS - Population Conservation, QC } \\
\text { Luc.Belanger@ec.gc.ca }\end{array}$ & $\begin{array}{l}\text { one of the authors of recent report of mowing impacts on roadside habitat quality (Belanger et } \\
\text { al. 2006); asked if he has any papers or data he'd be willing to share with us about bird mortality } \\
\text { related to roadside mowing; Luc responded by forwarding request to one of the other authors } \\
\text { (Benoit Jobin) and asking him to respond (see below) }\end{array}$ \\
\hline $\begin{array}{l}\text { Benoit Jobin, SAR Biologist } \\
\text { EC - Ecosystem Conservation, Sainte- } \\
\text { Foy }\end{array}$ & $\begin{array}{l}\text { "The project aimed at evaluating bird use of roadside rights-of-way along } 3 \text { highway sections in } \\
\text { southern Québec. We did not look at the actual effect of mowing on birds. We were looking a } \\
\text { the effect on bird use and habitat characteristics of a reduction of the frequency of mowing .." }\end{array}$ \\
\hline
\end{tabular}




\begin{tabular}{|c|c|}
\hline Benoit.Jobin@ec.gc.ca & nest searches in the roadside habitat found a single nest - mallard \\
\hline $\begin{array}{l}\text { Brenda Dale, Wildlife Population } \\
\text { Biologist, EC - Population Assessment } \\
\text { Unit, Edmonton } \\
\text { Brenda.Dale@EC.gc.ca }\end{array}$ & $\begin{array}{l}\text { sent a large number of papers/reports about various impacts on nesting success (e.g., habitat } \\
\text { fragmentation/connectivity, vegetation structure), roadkill, invasive species, effects of haying on } \\
\text { habitat quality, effects of roads/trails on bird community structure, and a number of papers to } \\
\text { help determine what species nest in roadside habitats; also provided expert opinion and review } \\
\text { of draft list of roadside nesting birds }\end{array}$ \\
\hline $\begin{array}{l}\text { Kathryn Lindsay, Senior Manager } \\
\text { EC - Habitat Landscape Conservation } \\
\text { \& Biodiversity Standards, Gatineau } \\
\text { Kathryn.Lindsay@ec.gc.ca }\end{array}$ & provided input for list of roadside nesting birds (sent Best et al. 1995), and reviewed draft list \\
\hline $\begin{array}{l}\text { Peter Blancher } \\
\text { EC - Ottawa } \\
\text { Ph: 613-998-7311 } \\
\text { peter.blancher@ec.gc.ca }\end{array}$ & $\begin{array}{l}\text { recommended talking to local birders to get information about roadside nesting birds; also } \\
\text { recommended Brenda Dale, EC - Edmonton for input; also provided guidance with the use of } \\
\text { the Partners in Flight Landbird Population Estimates Database }\end{array}$ \\
\hline $\begin{array}{l}\text { Don McNicol, Head } \\
\text { EC - Population Assessment, Ottawa } \\
\text { Ph: 613-949-8266 } \\
\text { Don.McNicol@ec.gc.ca }\end{array}$ & $\begin{array}{l}\text { NatureServe web site provides GIS layers for bird breeding ranges } \\
\text { http://www.natureserve.org/getData/animalData.jsp }\end{array}$ \\
\hline $\begin{array}{l}\text { Connie Downes, Landbird Surveys } \\
\text { Biologist; EC - Species Abundance and } \\
\text { Distribution, Ottawa } \\
\text { connie.downes@ec.gc.ca }\end{array}$ & $\begin{array}{l}\text { described Breeding Bird Survey methods, and limitations for use in calculating nest densities; } \\
\text { sent an English translation of Executive Summary for Belanger et al. } 2006\end{array}$ \\
\hline $\begin{array}{l}\text { Becky Stewart, Maritimes Breeding } \\
\text { Bird Atlas Coordinator, Sackville } \\
\text { bstewart@bsc-eoc.org }\end{array}$ & $\begin{array}{l}\text { requested information about nest density estimates for savannah sparrow and song sparrow as } \\
\text { these are not represented in the CBBC (Kennedy et al. 1999); "I'm afraid it hasn't already been } \\
\text { summarized--all I can provide you with is the raw data from Nature Counts on our website } \\
\text { www.mba-aom.ca" }\end{array}$ \\
\hline
\end{tabular}




\section{APPENDIX B: MODELLING APPROACH}

Simulation is a popular approach for estimating uncertainty when the theoretical calculations are complex. When the statistic of interest is a function of several other random variables, the variance calculations can be very difficult to solve theoretically. Monte Carlo sampling is one alternative approach for multi-dimensional numerical integration (Efron and Tibshirani 1994), which takes a brute force approach to solving such problems.

Total incidental take resulting from road maintenance activities depends on many variables, and each of these variables has uncertainty. Estimates of total take and associated uncertainty must incorporate the uncertainty in each of the component variables; we used a simulation approach to do this. As with any model, a number of assumptions were made. While no model is perfect, a simulation model is a useful tool for bounding a problem and identifying knowledge gaps. Simply proposing a model often provokes debate and helps to identify new research questions.

We followed the general framework used by Tews et al. (2009) in their assessment of incidental take associated with agricultural activities. Essentially the model compares the date of a disturbance activity to the presence of breeding birds (adults, eggs, hatchlings) within the disturbance area. In a deterministic model, all of the inputs are fixed (i.e., do not include any uncertainty). A stochastic simulation model typically uses a combination of fixed and random variables, but must have at least one random term by definition. In both cases an assumption is made about the value of the input variable and the distribution and the degree of uncertainty. However in the case of the fixed variable we are implicitly assuming that there is no uncertainty. Choosing which variables to fix and which to treat as random is somewhat subjective. However, we followed two general principles: 1 ) incorporate uncertainty for the terms most likely to affect variability in take; and 2) keep to simple assumptions where limited data were available, rather than trying to assess uncertainty.

The ability to estimate uncertainty is one of the key advantages to using a stochastic simulation model to estimate take. There are several different approaches to estimating uncertainty. First, if the observed results (e.g., 1000 simulation outcomes) are approximately normal or can be transformed so that they are approximately normal, then a traditional normal confidence interval can be calculated. Another approach which requires no assumption about distribution is a percentile interval. If the distribution is normal, the percentile interval will be the same as the normal confidence interval, but if the normal assumption was violated, the percentile interval is more reliable (Efron and Tibshirani, 1994).

Tews et al. (2009) incorporated uncertainty in two variables: 1) a random nest initiation date each year; and 2) a random mowing date. We also chose to include two random variables. Like Tews et al. (2009), we use a random start date for nesting. We considered incorporating uncertainty in the disturbance inputs, but ended up rejecting this idea. The disturbance data we were able to reliably obtain on the scale necessary for a Canada-wide estimate were very coarse - simply a proportion of maintenance effort by month (see Appendix C: Roads). Additionally, the timing of maintenance operations was driven not only by environmental conditions, but also by contracting cycles and contractor availability. Instead, we decided to include uncertainty in the estimate of nest density, as populations are known to fluctuate between years with environmental covariates or population cycles, and our confidence in the density estimates is not perfect (i.e., assuming zero uncertainty is unreasonable). While the various life-history variables of clutch size, sex ratio, nests per female, and egg to fledge timing 
are likely to vary on a local scale, we did not include uncertainty in these variables because limited data were available and we weren't interested in local scale estimates. For example, if we assume a clutch size of four eggs, but in reality some nests have three and some have five, it won't affect the calculations of take at the regional scale. We are interested in the variability in annual take at the regional scale, not how take varies from one hectare to the next.

\section{Adding Uncertainty}

Like Tews et al. (2009), we use a random nest initiation date which is drawn from a uniform (a, b) distribution with the values of parameters $a$, b defined by the user. Figure 2 shows an example of 1000 draws from a random uniform $(91,105)$ for killdeer in Ontario.

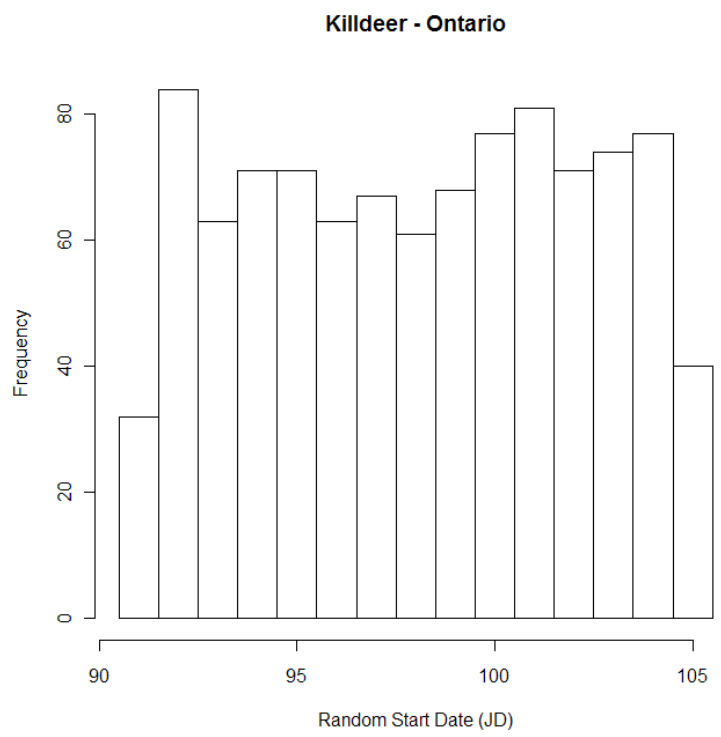

Figure 2: $\quad$ Example of 1000 random nest start dates drawn from a random uniform $(91,105)$ draws used in the simulation for Ontario killdeer. The lower frequencies at the end point are a consequence of the fact that after we take the random uniform draw, we round to the nearest Julian Day (i.e., integer) and the endpoints (91 \& 105) have no probability in the continuous distribution.

Incorporating uncertainty in the bird densities was more challenging due to the nature and quality of the data. Density estimates were obtained from Kennedy et al. (1999) as birds per $\mathrm{km}^{2}$ by habitat type. Where data were not available we used densities from the nearest neighbouring province/territory for which there were data. Where multiple values were available, we used the mean value (see Appendix C:Birds). The non-zero estimates vary from 0.1-314 birds $/ \mathrm{km}^{2}$. While count or density data are often approximated by a Poisson distribution, this distribution is sensitive to the units (ha, $\mathrm{km}^{2}, 1000 \mathrm{~km}^{2}$ ). Simulated Poisson data generates integer values so, at lower abundances, it results in many zeros or $1 \mathrm{~s}$ with nothing in between. This is realistic at the local scale, but is not the scale at which we have set up the simulation. For the purpose of the simulation, the parameter of interest is variation of annual density. A uniform distribution is too simple an assumption for densities, and a normal distribution is not appropriate as it may result in negative densities, especially for the lower density species. Abundance is often well approximated by a log-normal distribution (Hilborn and Mangel 1997). The lognormal distribution has characteristics similar to those of the Poisson distribution, but is continuous. By definition the $\mathrm{X}=\ln (\mathrm{Y}) \sim$ Normal (mu, sigma), if $\mathrm{Y} \sim \operatorname{lognormal~(mu,~sigma).~Where~mu,~and~sigma~refer~to~the~mean~and~standard~}$ deviation of $\mathrm{X}$ (i.e., the normal distribution). The mean and standard deviation of $\mathrm{Y}$ (i.e., the lognormal distribution) are related to but not equal to mu and sigma. For each run of the simulation, we drew from a random lognormal distribution with mu=log(density). 
Without better information, we assumed the variability was proportional to the mean and used sigma $=0.1^{*} \log$ (density) (i.e., CV of $10 \%$ on the log scale). As an example, the user input density for killdeer in shrubland in Ontario was $15.4 \mathrm{birds} / \mathrm{km}^{2}$. Figure 3a illustrates a realistic distribution for density (e.g., non-negative, skewed to the right), however skewed distributions are more difficult to analyze and so we can transform the data to something familiar (i.e., a normal distribution) as shown in Figure 3b).
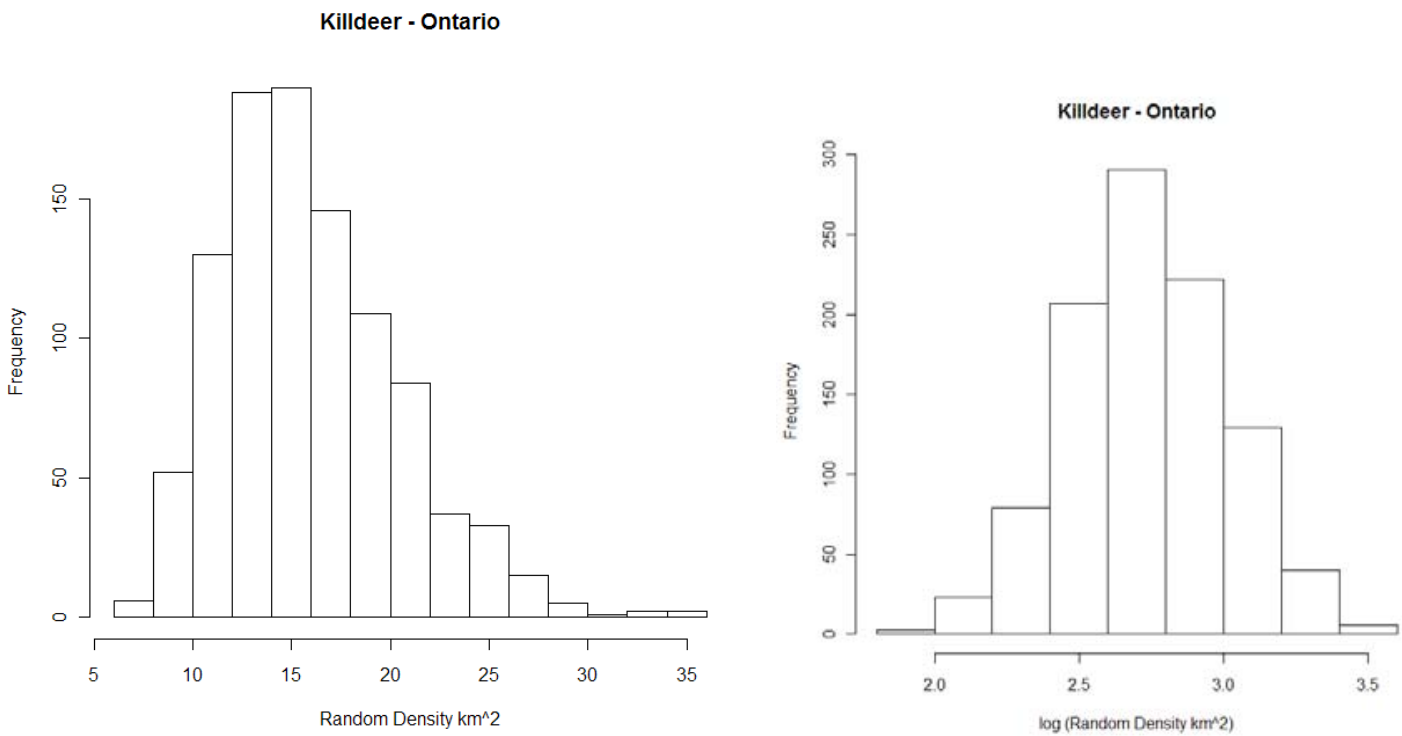

Figure 3: a) shows 1000 draws from a lognormal distribution with mu=log(15.4) and sigma=.1* $\log (15.4)$, notice the median of the distribution $=15.4$ and the distribution is skewed; b) shows the same data after taking a natural log transformation, notice the mean of the distribution $=\log (15.4)$ and the distribution is no longer skewed.

\section{Model Structure}

The model has an annual time scale, so each run of the simulation estimates the total take for one year. The spatial scale is flexible and depends on the user defined 'Regions'. For this report, we have treated each Province as a Region, and so the model estimates the annual take for a Province (Region). Estimates are generated separately for every focal species. Separate model inputs are required for every species and region.

The model script was written in several stand alone sections. A simple function (take.fcn) at the heart of the model calculates the annual take for a generic scenario (i.e., any land clearing activity). Several other functions calculate the necessary inputs specific to the 'roadmaintenance' tally and loop through all scenarios of interest to the road maintenance tally. The advantage of this segmented approach is that the take.fcn function may be used to estimate the annual take for any disturbance activity; it is not just limited to 'road-maintenance'.

\section{Model Inputs}

File name

Description 
LifeHistorylnputs.csv

Disturb-data.csv

TotalRoadsRegion.csv

RoadsBySpecies.csv

Discount.csv
For every Species, Region, and Habitat class this file contains:

o DensityKm - Density $/ \mathrm{km}^{\wedge} 2$ of adult birds

o ClutchSize - Mean Clutch Size

o FirstEggMean - earliest breeding date (in Julian days)

0 rangeFirstegg - range in dates for the first egg laid

o EggWindow - Length of breeding season (days between the first egg laid and the last egg laid)

o EggFledge - incubation time + nestling time

o P.Female - proportion of females in the population

o NumNestFemale - number of nests per female (per year)

o B_affected-proportion of eggs/nestlings killed given that they are present when a disturbance occurs

o P_affected - proportion of the area that is actually disturbed (not currently in use, but might be useful if different types of disturbance affect areas differently

For every Region, this file contains:

o The breakdown by month for the disturbance (i.e., \% by month) For every Region, this file contains:

o Total_Roads - The total length of roads in $\mathrm{km}$

o Total_Ha_Disturbed - The total area $(\mathrm{Ha})$ affected by road maintenance activity

For every Species, Region, and Habitat class, this file contains:

o Roads - the length of roads $(\mathrm{km})$ in the breeding range intersecting each habitat type

For every Species and Habitat class (both surrounding area and roadside):

o Discount: a multiplier to account for possibility that productivity of roadside habitat may vary and may differ depending on the surrounding habitat

\section{Script Files}

Script files are shown in grey headings, with embedded functions listed in the left column and described in the right column.

\begin{tabular}{|l|l|}
\hline Bird_functions.r & $\begin{array}{l}\text { This file contains several stand-alone functions that are necessary for } \\
\text { estimating take. }\end{array}$ \\
\hline Egg.density() & $\begin{array}{l}\text { Calculates the density of eggs given: adult density, clutch size, proportion of } \\
\text { females, nests per female, and a discount value if specified. Each of these } \\
\text { variables is user input, but initially we assumed that: the proportion of } \\
\text { females }=.5 \text { and only 1 nest per female per year. }\end{array}$ \\
\hline p.present() & $\begin{array}{l}\text { Calculates the proportion of the population of eggs and nestlings are present } \\
\text { on a given Julian day given: } \\
0 \text { Julian day, FirstEgg, EggWindow, EggFledge }\end{array}$ \\
\hline Disturbance() & $\begin{array}{c}\text { Function that takes in: } \\
\text { o a vector (length 12) with the pct area affected by month } \\
0 \text { h.overlap - total hectares disturbed in the breeding area, } \\
\text { o p.affected - set to 1 but was meant to allow for situations where only } \\
\text { 1/2 the roads are mowed each year } \\
\rightarrow \text { outputs a matrix of hectares disturbed by julian day for the entire year }\end{array}$ \\
\hline Area() & Calculates the hectares disturbed within each habitat, given: \\
\hline
\end{tabular}




\begin{tabular}{|c|c|}
\hline & $\begin{array}{l}\text { o total km of roads in the region } \\
\text { o km of road by habitat type, within the breeding range } \\
\text { o total hectares disturbed } \\
\text { This function translates between the road information in km to area disturbed } \\
\text { in hectares. We assume the disturbance is applied equally to all roads. We } \\
\text { use the proportion of roads within the breeding area to calculate the } \\
\text { hectares disturbed within the breeding area. We assume that the } \\
\text { disturbance is applied to each habitat type in the same proportion that we } \\
\text { observe roads by habitat type (within the breeding range). }\end{array}$ \\
\hline Discount.fen() & $\begin{array}{l}\text { Calculates the hectares disturbed within each habitat sub-category, given: } \\
\text { - The hectares disturbed within each habitat type, from Area() } \\
\text { - Assumptions about roadside habitat type and quality (Table 3) }\end{array}$ \\
\hline Take.fcn() & $\begin{array}{l}\text { Requires: egg.density(); p.present () } \\
\text { Calculates take for a single year for a specific: species, region, and habitat } \\
\text { type inputs: FirstEgg,AdultDens, EggWindow, EggFledge, p.female, NPF, } \\
\text { EggsPerNest, Rel.effort, H.overlap (hectares disturbed in breeding range), } \\
\text { P.affected, B.affected } \\
\text { Outputs: } \\
\text { o Calculates take by day and reports total take for the year } \\
\text { Note: This function can be run as a stand-alone function for any disturbance } \\
\text { type, species, region of interest. It is not restricted to the 'road-maintenance' } \\
\text { tally. }\end{array}$ \\
\hline Bird_overall_fcn.r & This file contains a single function. \\
\hline Overall.fen & $\begin{array}{l}\text { Inputs: } \\
\text { o Region } \\
\text { o Species } \\
\text { o n, number of runs } \\
\text { o noise (as a percent of the mean on the log scale) } \\
\text { This function calculates the annual take for a species and region. Each run } \\
\text { represents a new year. Within a year and region we assume the start date } \\
\text { and densities are not independent. We use the same start date for all habitat } \\
\text { types within a region, species, and year. We let the density change annually, } \\
\text { but maintain the same relative density between habitat types. } \\
\text { Outputs: } \\
\text { interim results (useful for ground truthing, de-bugging) these results } \\
\text { display the hectares disturbed within the breeding area for each sub- } \\
\text { category. } \\
\text { o simulation results - each row represents a single year or run. There } \\
\text { is a column for each habitat sub-category in case it is of interest to } \\
\text { compare among categories. There is also a summary column } \\
\text { documenting the annual take across all categories. Finally there are } \\
\text { two columns to record the two randomly generated variables: first } \\
\text { day \& density. }\end{array}$ \\
\hline
\end{tabular}




\begin{tabular}{|l|l|}
\hline Birds_run.r & $\begin{array}{l}\text { The function that actually runs the code \& calculates take for several habitat } \\
\text { types within a region. }\end{array}$ \\
\hline & $\begin{array}{r}\text { Steps: } \\
\text { 1) set working directory } \\
\text { 2) read in input files } \\
\text { 3) run overall.fcn for each species and region of interest }\end{array}$ \\
\hline Birds_results.r & Summarizes the simulated results: histograms, Cl's \\
\hline & $\begin{array}{l}\text { Calculates quantile confidence intervals as well as confidence intervals } \\
\text { assuming a log normal distribution. These are then output to a file. } \\
\text { Histograms for the simulation results are also stored to a file. }\end{array}$ \\
\hline
\end{tabular}

Steps for a single run of the road-maintenance simulation (details in the table above):

1) Pull the relevant inputs (i.e., information for the current species and region) from the input files.

2) Calculate the area in hectares disturbed within each habitat class ${ }^{3}$, using the Area() function.

3) Calculate the area in hectares disturbed within each habitat sub-category ${ }^{4}$ (e.g., forestgrass, forest except grass @ roadside) and look up the associated discount multiplier; an example of interim results is shown in Table 13. Later in the model, the information in this table will be used to discount the densities in each of the habitat class sub-categories and to determine how many eggs/nestlings are present in each habitat type. This table is produced by the Discount.fcn().

Table 13: $\quad$ Example of interim results of the Discount.fen() for killdeer in Ontario.

$\begin{array}{lllrrrr}\text { Species } & \text { hab.class } & \text { new.hab } & \text { Discount } & \begin{array}{c}\text { Hectares } \\ \text { Disturbed }\end{array} & \text { DensityKm } & \text { DensityHa } \\ \text { Killdeer } & \text { grass } & \text { forest.grass } & 0.1 & 7364.8 & 10.16 & 0.1016 \\ \text { Killdeer } & \text { grass } & \text { grass.grass } & 1 & 16946.9 & 10.16 & 0.1016 \\ \text { Killdeer } & \text { grass } & \text { wetland.grass } & 1 & 300.7 & 10.16 & 0.1016 \\ \text { Killdeer } & \text { shrub } & \text { forest.shrub } & 0.1 & 7364.8 & 15.4 & 0.154 \\ \text { Killdeer } & \text { shrub } & \text { shrub.shrub } & 1 & 261.0 & 15.4 & 0.154 \\ \text { Killdeer } & \text { wetland } & \text { wetland.wetland } & 1 & 300.7 & 14.14 & 0.1414\end{array}$

4) Look up the relative effort, i.e., the proportion of maintenance that occurs during each month.

5) Randomly select a 'first egg date' from a uniform distribution with user input start and end dates.

6) Pick out the maximum density across habitat types within the species and region.

7) Randomly select an annual estimate of density, from a lognormal distribution based on the maximum density from the previous step (e.g., lognormal(mu=log(max.dens), sigma $=.1^{*} \log (\max$. dens $\left.)\right)$.

${ }^{3}$ Habitat class refers to the general habitat class with which the roads intersect as identified from the GIS exercise.

${ }^{4}$ Habitat class sub-category refers to the general habitat class as well as the local roadside habitat. For example: forest-grass: is roadside grassland habitat that is running through a forest, whereas grassgrass: is roadside grassland habitat running through a grassland. 
8) We assume that the density in different habitat types within a region are correlated (e.g., in low density years, they are all low density). Calculate the density for the other habitat types so as to maintain the same relative size. In other words, allow density to vary among years, but ensure that within a year the relative density in each habitat is maintained.

9) For each habitat type within the Region, call the generic take.fcn() which calculates annual take by habitat sub-category, Region, and species.

Take.fcn(), this generic function can be applied to any disturbance activity:

10) Uses the disturbance() function to determine how many hectares are disturbed each day of the year. Assumes that the monthly effort is spread equally among days in the month.

11) Uses p.present() to estimate the density of birds vulnerable to destruction each day of the year. We assume the proportion of eggs for the year can be fit by a straight line between the first egg date and the last day an egg is laid (i.e., the proportion $=0$ before the first egg is laid, and =1 after the last egg is laid). This differs slightly from Tews et al. (2009) who used a regression model with real data to predict this curve. However, upon review the data were all fit adequately with a simple straight-line model which suggests that a straight-line model is a reasonable assumption to begin with. If data become available to suggest a different shape is more appropriate, it would be easy to update the code accordingly. Once the proportion of eggs by day is determined, the presence of eggs and/or nestlings for each day can be calculated based on the user input egg to fledge timing. It is then a simple matter to check any individual day to see whether or not any eggs or nestlings are available to be harmed. We assumed a fixed egg to fledge time. Although Tews et al. (2009) use a second regression curve to predict fledging behaviour, in all but one case they simply used the exact same fit as for the egg-laying; this is essentially the same as simply using a fixed egg to fledge time as we have done.

12) The number of hectares disturbed each day is then compared with the density of vulnerable birds on the same day. The daily density of vulnerable birds is multiplied by the daily hectares disturbed to estimate the daily take. The take for all days in the year is summed.

13) The annual estimate of take by habitat type, species, and region is saved and a new run begins.

14) The estimates for many runs are then summarized to provide an estimate of the total annual take and associated uncertainty. We report the median along with the $2.5^{\text {th }}$ and $97.5^{\text {th }}$ percentiles (Figure 4). 


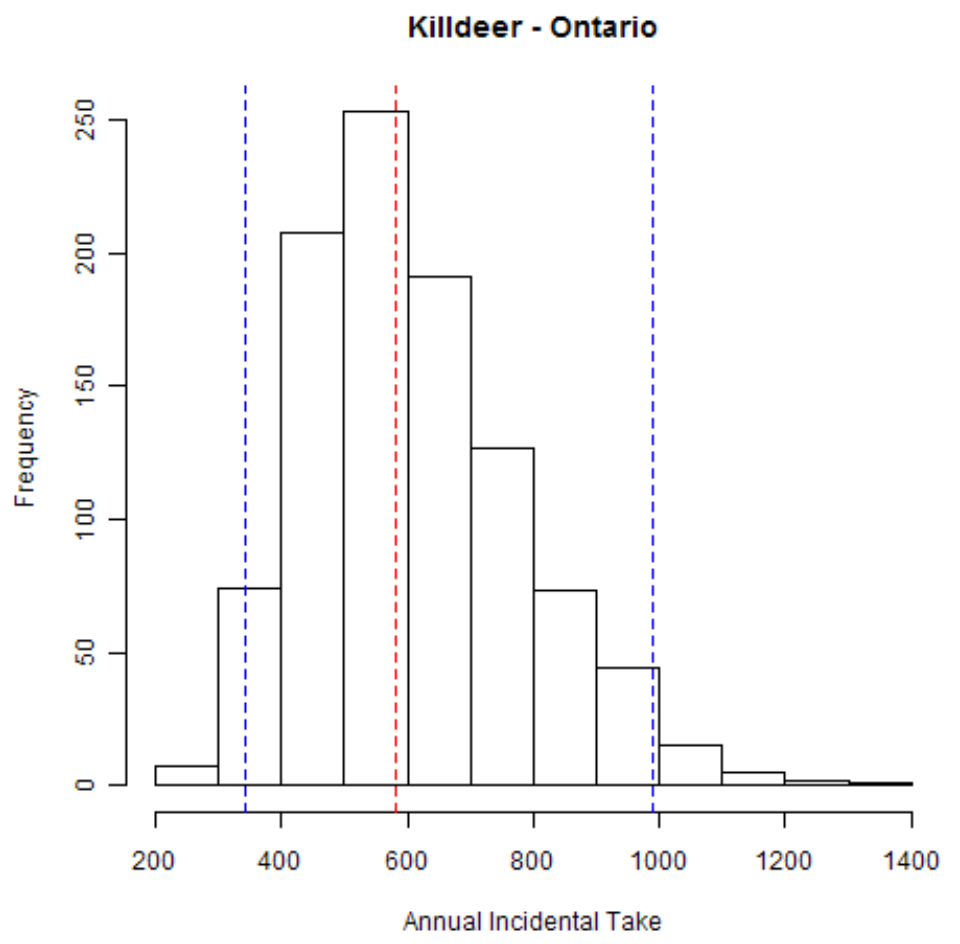

Figure 4: $\quad$ Summary of 1000 outcomes from the simulation model for Ontario killdeer. Each run results in an annual estimate of take. The red line represents the median or the $50^{\text {th }}$ percentile from those 1000 simulations. The blue lines represent the $2.5^{\text {th }}$ and $97.5^{\text {th }}$ percentiles respectively.

\section{Example of model output for killdeer}

Estimates are reported by province or territory. In general, the observed results were approximately lognormally distributed, and were transformed to normalize them. Both nonparametric percentile intervals and normal confidence intervals are provided (based on the log transformed results) (Table 14). For this report we have shown the non-parametric results for each Region as they require no assumptions about distribution.

Table 14: $\quad$ Example of model output for killdeer. Both non-parametric percentile intervals and parametric confidence intervals (assuming lognormal distribution) are reported. LB = lower bound; UB = upper bound.

\begin{tabular}{llcccccc}
\hline & & \multicolumn{3}{c}{$\begin{array}{l}\text { Median \& 95\% Percentile } \\
\text { intervals }\end{array}$} & \multicolumn{3}{c}{$\begin{array}{l}\text { Mean \& 95\% Confidence Intervals } \\
\text { (assuming log(results) normal }\end{array}$} \\
\hline Species & Region & $\begin{array}{l}\text { Median } \\
(50 \%)\end{array}$ & $\begin{array}{l}\text { LB } \\
(2.5 \%)\end{array}$ & $\begin{array}{l}\text { UB } \\
(97.5 \%)\end{array}$ & mean & LB & UB \\
\hline Killdeer & BC & 6.9 & 4.8 & 9.9 & 6.9 & 4.7 & 10.1 \\
Killdeer & Yukon & 0.1 & 0.0 & 0.0 & 0.0 & 0.0 & 0.0 \\
Killdeer & NW Territories & & & & & & \\
Killdeer & Alberta & 27.5 & 19.8 & 37.7 & 27.4 & 19.8 & 37.9 \\
Killdeer & Saskatchewan & 1282.6 & 802.7 & 2019.2 & 1288.5 & 810.6 & 2048.3 \\
Killdeer & Manitoba & 129.4 & 106.2 & 160.5 & 129.6 & 104.9 & 160.1 \\
Killdeer & Ontario & 580.2 & 341.3 & 988.9 & 584.8 & 339.5 & 1007.2 \\
Killdeer & Quebec & 0.2 & 0.2 & 0.2 & 0.2 & 0.2 & 0.2
\end{tabular}




\begin{tabular}{|c|c|c|c|c|c|c|c|}
\hline Killdeer & NB & 0.0 & 0.0 & 0.0 & 0.0 & 0.0 & 0.0 \\
\hline Killdeer & Nova Scotia & 0.0 & 0.0 & 0.0 & 0.0 & 0.0 & 0.0 \\
\hline Killdeer & PEI & 0.0 & 0.0 & 0.0 & 0.0 & 0.0 & 0.0 \\
\hline Killdeer & NL & & & & & & \\
\hline Killdeer & Nunavut & & & & & & \\
\hline
\end{tabular}




\section{APPENDIX C: MODEL INPUTS}

\section{Bird Breeding and Density Inputs}

The following table contains the bird breeding and density data used in the simulation model. Density values by habitat type are from Kennedy et al. (1999), and breeding information is from Peck and James (1983, 1987).

\begin{tabular}{|c|c|c|c|c|c|c|c|c|c|}
\hline Species & Prov. & $\begin{array}{c}\text { Nesting } \\
\text { Habitat }\end{array}$ & $\begin{array}{l}\text { Birds } \\
\text { /km }^{2}\end{array}$ & $\begin{array}{c}\text { In } \\
\text { Range? }\end{array}$ & $\begin{array}{l}\text { Clutch } \\
\text { Size }\end{array}$ & $\begin{array}{c}\text { First Egg } \\
\text { Egg (Date) }\end{array}$ & $\begin{array}{c}\text { Min } \\
\text { Incubation } \\
\text { Time (d) }\end{array}$ & $\begin{array}{c}\text { Max } \\
\text { Incubation } \\
\text { Time (d) } \\
\end{array}$ & $\begin{array}{c}\text { Mean } \\
\text { Nestling } \\
\text { Period }\end{array}$ \\
\hline KIШ & $\mathrm{BC}$ & $F$ & 0 & $y$ & 4 & April 1 & 24 & 30 & 2 \\
\hline KIШ & $\mathrm{BC}$ & $\mathrm{S}$ & 3.6 & $y$ & 4 & April 1 & 24 & 30 & 2 \\
\hline KIШ & $\mathrm{BC}$ & G & 0 & $y$ & 4 & April 1 & 24 & 30 & 2 \\
\hline KIШ & $\mathrm{BC}$ & W & 0 & $y$ & 4 & April 1 & 24 & 30 & 2 \\
\hline KIL & $A B$ & $\mathrm{~F}$ & 0 & $y$ & 4 & April 1 & 24 & 30 & 2 \\
\hline KIШ & $A B$ & $\mathrm{~S}$ & 3.6 & $y$ & 4 & April 1 & 24 & 30 & 2 \\
\hline KIL & $A B$ & G & 0 & $y$ & 4 & April 1 & 24 & 30 & 2 \\
\hline KIL & $A B$ & W & 0 & $y$ & 4 & April 1 & 24 & 30 & 2 \\
\hline KIL & SK & $F$ & 0 & $y$ & 4 & April 1 & 24 & 30 & 2 \\
\hline KIL & SK & $\mathrm{S}$ & 0 & $y$ & 4 & April 1 & 24 & 30 & 2 \\
\hline KIШ & SK & G & 8.73 & $y$ & 4 & April 1 & 24 & 30 & 2 \\
\hline KIப & SK & W & 0 & $y$ & 4 & April 1 & 24 & 30 & 2 \\
\hline KIШ & $\mathrm{MB}$ & $F$ & 0 & $y$ & 4 & April 1 & 24 & 30 & 2 \\
\hline KIப & $\mathrm{MB}$ & $\mathrm{S}$ & 0 & $y$ & 4 & April 1 & 24 & 30 & 2 \\
\hline KIШ & $\mathrm{MB}$ & G & 2.85 & $y$ & 4 & April 1 & 24 & 30 & 2 \\
\hline KIL & $\mathrm{MB}$ & W & 0 & $y$ & 4 & April 1 & 24 & 30 & 2 \\
\hline KIШ & ON & $F$ & 3.92 & $y$ & 4 & April 1 & 24 & 30 & 2 \\
\hline KIШ & ON & $S$ & 15.4 & $y$ & 4 & April 1 & 24 & 30 & 2 \\
\hline KIШ & ON & G & 10.16 & $y$ & 4 & April 1 & 24 & 30 & 2 \\
\hline KIШ & ON & W & 14.14 & $y$ & 4 & April 1 & 24 & 30 & 2 \\
\hline KIШ & QC & $F$ & 0 & $y$ & 4 & April 1 & 24 & 30 & 2 \\
\hline KIШ & QC & $\mathrm{S}$ & 0 & $y$ & 4 & April 1 & 24 & 30 & 2 \\
\hline KIப & QC & $G$ & 0 & $y$ & 4 & April 1 & 24 & 30 & 2 \\
\hline KIL & QC & W & 1 & $y$ & 4 & April 1 & 24 & 30 & 2 \\
\hline KIШ & NB & $F$ & 0 & $y$ & 4 & April 1 & 24 & 30 & 2 \\
\hline KIL & NB & $S$ & 0 & $y$ & 4 & April 1 & 24 & 30 & 2 \\
\hline
\end{tabular}




\begin{tabular}{|c|c|c|c|c|c|c|c|c|c|}
\hline Species & Prov. & $\begin{array}{c}\text { Nesting } \\
\text { Habitat }\end{array}$ & $\begin{array}{l}\text { Birds } \\
/ \mathbf{k m}^{2}\end{array}$ & $\begin{array}{c}\text { In } \\
\text { Range? }\end{array}$ & $\begin{array}{c}\text { Clutch } \\
\text { Size }\end{array}$ & $\begin{array}{c}\text { First Egg } \\
\text { Egg (Date) }\end{array}$ & $\begin{array}{c}\text { Min } \\
\text { Incubation } \\
\text { Time (d) }\end{array}$ & $\begin{array}{c}\text { Max } \\
\text { Incubation } \\
\text { Time (d) }\end{array}$ & $\begin{array}{c}\text { Mean } \\
\text { Nestling } \\
\text { Period }\end{array}$ \\
\hline KIL & $\mathrm{NB}$ & $G$ & 0 & $y$ & 4 & April 1 & 24 & 30 & 2 \\
\hline KIL & NB & w & 1.5 & $y$ & 4 & April 1 & 24 & 30 & 2 \\
\hline КाШ & NS & $\mathrm{F}$ & 0 & $y$ & 4 & April 1 & 24 & 30 & 2 \\
\hline КाШ & NS & $\mathrm{s}$ & 0 & $y$ & 4 & April 1 & 24 & 30 & 2 \\
\hline КाШ & NS & G & 0 & $y$ & 4 & April 1 & 24 & 30 & 2 \\
\hline KIL & NS & W & 1.5 & $y$ & 4 & April 1 & 24 & 30 & 2 \\
\hline KIL & P曰 & F & 0 & $y$ & 4 & April 1 & 24 & 30 & 2 \\
\hline KIL & P曰 & S & 0 & $y$ & 4 & April 1 & 24 & 30 & 2 \\
\hline KIL & P巨 & G & 0 & $y$ & 4 & April 1 & 24 & 30 & 2 \\
\hline KIL & PE & W & 1.5 & $y$ & 4 & April 1 & 24 & 30 & 2 \\
\hline KIШ & NL & F & 0 & $y$ & 4 & April 1 & 24 & 30 & 2 \\
\hline KIШ & NL & $s$ & 0 & $y$ & 4 & April 1 & 24 & 30 & 2 \\
\hline KIL & $\mathrm{NL}$ & $G$ & 0 & $y$ & 4 & April 1 & 24 & 30 & 2 \\
\hline KIL & $\mathrm{NL}$ & w & 1.5 & $y$ & 4 & April 1 & 24 & 30 & 2 \\
\hline KIL & $\mathrm{NU}$ & $\mathrm{F}$ & 0 & $\mathrm{n}$ & 4 & April 1 & 24 & 30 & 2 \\
\hline KIL & $\mathrm{NU}$ & s & 0 & $\mathrm{n}$ & 4 & April 1 & 24 & 30 & 2 \\
\hline KIШ & $\mathrm{NU}$ & G & 0 & $\mathrm{n}$ & 4 & April 1 & 24 & 30 & 2 \\
\hline KIL & $\mathrm{NU}$ & w & 0 & $\mathrm{n}$ & 4 & April 1 & 24 & 30 & 2 \\
\hline KIШ & NT & $\mathrm{F}$ & 0 & $y$ & 4 & April 1 & 24 & 30 & 2 \\
\hline КІL & $N T$ & $\mathrm{~s}$ & 0 & $y$ & 4 & April 1 & 24 & 30 & 2 \\
\hline KIL & NT & G & 0 & $y$ & 4 & April 1 & 24 & 30 & 2 \\
\hline KIL & NT & w & 1.5 & $y$ & 4 & April 1 & 24 & 30 & 2 \\
\hline KIL & $\mathrm{YT}$ & $\mathrm{F}$ & 0 & $y$ & 4 & April 1 & 24 & 30 & 2 \\
\hline KIL & $\mathrm{YT}$ & s & 0 & $y$ & 4 & April 1 & 24 & 30 & 2 \\
\hline KIL & $\mathrm{YT}$ & $G$ & 0 & $y$ & 4 & April 1 & 24 & 30 & 2 \\
\hline KIL & $\mathrm{YT}$ & w & 1.5 & $y$ & 4 & April 1 & 24 & 30 & 2 \\
\hline SASP & $\mathrm{BC}$ & $\mathrm{F}$ & 0 & $y$ & 4 & April 27 & 9 & 13 & 9 \\
\hline SASP & $\mathrm{BC}$ & s & 22.2 & $y$ & 4 & April 27 & 9 & 13 & 9 \\
\hline SASP & $B C$ & G & 0 & $y$ & 4 & April 27 & 9 & 13 & 9 \\
\hline SASP & $\mathrm{BC}$ & W & 0 & $y$ & 4 & April 27 & 9 & 13 & 9 \\
\hline SASP & $A B$ & $\mathrm{~F}$ & 0.1 & $y$ & 4 & April 27 & 9 & 13 & 9 \\
\hline SASP & $A B$ & $s$ & 32 & $y$ & 4 & April 27 & 9 & 13 & 9 \\
\hline SASP & $A B$ & $G$ & 19.57 & $y$ & 4 & April 27 & 9 & 13 & 9 \\
\hline
\end{tabular}




\begin{tabular}{|c|c|c|c|c|c|c|c|c|c|}
\hline Species & Prov. & $\begin{array}{c}\text { Nesting } \\
\text { Habitat }\end{array}$ & $\begin{array}{l}\text { Birds } \\
/ \mathbf{k m}^{2}\end{array}$ & $\begin{array}{c}\text { In } \\
\text { Range? }\end{array}$ & $\begin{array}{c}\text { Clutch } \\
\text { Size }\end{array}$ & $\begin{array}{c}\text { First Egg } \\
\text { Egg (Date) }\end{array}$ & $\begin{array}{c}\text { Min } \\
\text { Incubation } \\
\text { Time (d) }\end{array}$ & $\begin{array}{c}\text { Max } \\
\text { Incubation } \\
\text { Time (d) }\end{array}$ & $\begin{array}{c}\text { Mean } \\
\text { Nestling } \\
\text { Period }\end{array}$ \\
\hline SASP & $A B$ & $w$ & 0 & $y$ & 4 & April 27 & 9 & 13 & 9 \\
\hline SASP & SK & $\mathrm{F}$ & 0 & $y$ & 4 & April 27 & 9 & 13 & 9 \\
\hline SASP & SK & $\mathrm{s}$ & 0 & $y$ & 4 & April 27 & 9 & 13 & 9 \\
\hline SASP & SK & G & 11.43 & $y$ & 4 & April 27 & 9 & 13 & 9 \\
\hline SASP & SK & w & 0 & $y$ & 4 & April 27 & 9 & 13 & 9 \\
\hline SASP & MB & F & 3.8 & $y$ & 4 & April 27 & 9 & 13 & 9 \\
\hline SASP & MB & S & 67.5 & $y$ & 4 & April 27 & 9 & 13 & 9 \\
\hline SASP & MB & G & 47.67 & $y$ & 4 & April 27 & 9 & 13 & 9 \\
\hline SASP & MB & W & 0 & $y$ & 4 & April 27 & 9 & 13 & 9 \\
\hline SASP & ON & F & 43.5 & $y$ & 4 & April 27 & 9 & 13 & 9 \\
\hline SASP & ON & $\mathrm{s}$ & 45.12 & $y$ & 4 & April 27 & 9 & 13 & 9 \\
\hline SASP & ON & G & 75.83 & $y$ & 4 & April 27 & 9 & 13 & 9 \\
\hline SASP & ON & w & 0 & $y$ & 4 & April 27 & 9 & 13 & 9 \\
\hline SASP & $\mathrm{QC}$ & $\mathrm{F}$ & 9.14 & $y$ & 4 & April 27 & 9 & 13 & 9 \\
\hline SASP & $\mathrm{QC}$ & s & 4 & $y$ & 4 & April 27 & 9 & 13 & 9 \\
\hline SASP & $\mathrm{QC}$ & G & 50 & $y$ & 4 & April 27 & 9 & 13 & 9 \\
\hline SASP & $\mathrm{QC}$ & w & 0 & $y$ & 4 & April 27 & 9 & 13 & 9 \\
\hline SASP & NB & $\mathrm{F}$ & 9 & $y$ & 4 & April 27 & 9 & 13 & 9 \\
\hline SASP & NB & s & 0 & $y$ & 4 & April 27 & 9 & 13 & 9 \\
\hline SASP & $\mathrm{NB}$ & $G$ & 0 & $y$ & 4 & April 27 & 9 & 13 & 9 \\
\hline SASP & $\mathrm{NB}$ & w & 83.33 & $y$ & 4 & April 27 & 9 & 13 & 9 \\
\hline SASP & NS & $\mathrm{F}$ & 9 & $y$ & 4 & April 27 & 9 & 13 & 9 \\
\hline SASP & NS & s & 0 & $y$ & 4 & April 27 & 9 & 13 & 9 \\
\hline SASP & NS & G & 0 & $y$ & 4 & April 27 & 9 & 13 & 9 \\
\hline SASP & NS & w & 83.33 & $y$ & 4 & April 27 & 9 & 13 & 9 \\
\hline SASP & PE & F & 9 & $y$ & 4 & April 27 & 9 & 13 & 9 \\
\hline SASP & PE & $s$ & 0 & $y$ & 4 & April 27 & 9 & 13 & 9 \\
\hline SASP & PE & $G$ & 0 & $y$ & 4 & April 27 & 9 & 13 & 9 \\
\hline SASP & PE & W & 83.33 & $y$ & 4 & April 27 & 9 & 13 & 9 \\
\hline SASP & $\mathrm{NL}$ & $\mathrm{F}$ & 9 & $y$ & 4 & April 27 & 9 & 13 & 9 \\
\hline SASP & NL & $S$ & 0 & $y$ & 4 & April 27 & 9 & 13 & 9 \\
\hline SASP & $\mathrm{NL}$ & G & 0 & $y$ & 4 & April 27 & 9 & 13 & 9 \\
\hline SASP & $\mathrm{NL}$ & w & 83.33 & $y$ & 4 & April 27 & 9 & 13 & 9 \\
\hline
\end{tabular}




\begin{tabular}{|c|c|c|c|c|c|c|c|c|c|}
\hline Species & Prov. & $\begin{array}{l}\text { Nesting } \\
\text { Habitat }\end{array}$ & $\begin{array}{l}\text { Birds } \\
/ \mathbf{k m}^{2}\end{array}$ & $\begin{array}{c}\text { In } \\
\text { Range? }\end{array}$ & $\begin{array}{c}\text { Clutch } \\
\text { Size }\end{array}$ & $\begin{array}{c}\text { First Egg } \\
\text { Egg (Date) }\end{array}$ & $\begin{array}{c}\text { Min } \\
\text { Incubation } \\
\text { Time (d) }\end{array}$ & $\begin{array}{c}\text { Max } \\
\text { Incubation } \\
\text { Time (d) }\end{array}$ & $\begin{array}{c}\text { Mean } \\
\text { Nestling } \\
\text { Period }\end{array}$ \\
\hline SASP & $\mathrm{NU}$ & $\mathrm{F}$ & 0 & $y$ & 4 & April 27 & 9 & 13 & 9 \\
\hline SASP & $\mathrm{NU}$ & $s$ & 20 & $y$ & 4 & April 27 & 9 & 13 & 9 \\
\hline SASP & $\mathrm{NU}$ & G & 8.65 & $y$ & 4 & April 27 & 9 & 13 & 9 \\
\hline SASP & $\mathrm{NU}$ & w & 0 & $y$ & 4 & April 27 & 9 & 13 & 9 \\
\hline SASP & NT & $\mathrm{F}$ & 0 & $y$ & 4 & April 27 & 9 & 13 & 9 \\
\hline SASP & NT & $\mathrm{s}$ & 20 & $y$ & 4 & April 27 & 9 & 13 & 9 \\
\hline SASP & NT & G & 8.65 & $y$ & 4 & April 27 & 9 & 13 & 9 \\
\hline SASP & NT & W & 0 & $y$ & 4 & April 27 & 9 & 13 & 9 \\
\hline SASP & $\mathrm{YT}$ & $\mathrm{F}$ & 0 & $y$ & 4 & April 27 & 9 & 13 & 9 \\
\hline SASP & $\mathrm{YT}$ & s & 20 & $y$ & 4 & April 27 & 9 & 13 & 9 \\
\hline SASP & YT & G & 8.65 & $y$ & 4 & April 27 & 9 & 13 & 9 \\
\hline SASP & $\mathrm{YT}$ & w & 0 & $y$ & 4 & April 27 & 9 & 13 & 9 \\
\hline SOSP & $\mathrm{BC}$ & $\mathrm{F}$ & 25.52 & $y$ & 4 & April 17 & 11 & 15 & 10 \\
\hline SOSP & $\mathrm{BC}$ & s & 19.7 & $y$ & 4 & April 17 & 11 & 15 & 10 \\
\hline SOSP & $\mathrm{BC}$ & $G$ & 0 & $y$ & 4 & April 17 & 11 & 15 & 10 \\
\hline SOSP & $\mathrm{BC}$ & w & 314.5 & $y$ & 4 & April 17 & 11 & 15 & 10 \\
\hline SOSP & $A B$ & $\mathrm{~F}$ & 10 & $y$ & 4 & April 17 & 11 & 15 & 10 \\
\hline SOSP & $A B$ & s & 8.5 & $y$ & 4 & April 17 & 11 & 15 & 10 \\
\hline SOSP & $A B$ & $G$ & 0 & $y$ & 4 & April 17 & 11 & 15 & 10 \\
\hline SOSP & $A B$ & w & 4.2 & $y$ & 4 & April 17 & 11 & 15 & 10 \\
\hline SOSP & SK & $\mathrm{F}$ & 53.35 & $y$ & 4 & April 17 & 11 & 15 & 10 \\
\hline SOSP & SK & s & 10 & $y$ & 4 & April 17 & 11 & 15 & 10 \\
\hline SOSP & SK & $G$ & 10.5 & $y$ & 4 & April 17 & 11 & 15 & 10 \\
\hline SOSP & SK & w & 3.08 & $y$ & 4 & April 17 & 11 & 15 & 10 \\
\hline SOSP & MB & $\mathrm{F}$ & 53.35 & $y$ & 4 & April 17 & 11 & 15 & 10 \\
\hline SOSP & MB & s & 10 & $y$ & 4 & April 17 & 11 & 15 & 10 \\
\hline SOSP & $\mathrm{MB}$ & G & 10.5 & $y$ & 4 & April 17 & 11 & 15 & 10 \\
\hline SOSP & $\mathrm{MB}$ & w & 3.08 & $y$ & 4 & April 17 & 11 & 15 & 10 \\
\hline SOSP & ON & F & 0 & $y$ & 4 & April 17 & 11 & 15 & 10 \\
\hline SOSP & ON & s & 96.47 & $y$ & 4 & April 17 & 11 & 15 & 10 \\
\hline SOSP & ON & G & 30.78 & $y$ & 4 & April 17 & 11 & 15 & 10 \\
\hline SOSP & ON & w & 43.35 & $y$ & 4 & April 17 & 11 & 15 & 10 \\
\hline SOSP & $\mathrm{QC}$ & $\mathrm{F}$ & 15.44 & $y$ & 4 & April 17 & 11 & 15 & 10 \\
\hline
\end{tabular}




\begin{tabular}{|c|c|c|c|c|c|c|c|c|c|}
\hline Species & Prov. & $\begin{array}{l}\text { Nesting } \\
\text { Habitat }\end{array}$ & $\begin{array}{l}\text { Birds } \\
\text { /km }^{2}\end{array}$ & $\begin{array}{c}\text { In } \\
\text { Range? }\end{array}$ & $\begin{array}{c}\text { Clutch } \\
\text { Size }\end{array}$ & $\begin{array}{c}\text { First Egg } \\
\text { Egg (Date) }\end{array}$ & $\begin{array}{c}\text { Min } \\
\text { Incubation } \\
\text { Time (d) }\end{array}$ & $\begin{array}{c}\text { Max } \\
\text { Incubation } \\
\text { Time (d) }\end{array}$ & $\begin{array}{c}\text { Mean } \\
\text { Nestling } \\
\text { Period }\end{array}$ \\
\hline SOSP & $\mathrm{QC}$ & $\mathrm{s}$ & 4 & $y$ & 4 & April 17 & 11 & 15 & 10 \\
\hline SOSP & $Q C$ & $\mathrm{G}$ & 0.1 & $y$ & 4 & April 17 & 11 & 15 & 10 \\
\hline SOSP & $\mathrm{QC}$ & w & 0 & $y$ & 4 & April 17 & 11 & 15 & 10 \\
\hline SOSP & NB & $\mathrm{F}$ & 37.5 & $y$ & 4 & April 17 & 11 & 15 & 10 \\
\hline SOSP & NB & $S$ & 0 & $y$ & 4 & April 17 & 11 & 15 & 10 \\
\hline SOSP & NB & G & 0 & $y$ & 4 & April 17 & 11 & 15 & 10 \\
\hline SOSP & NB & W & 9.92 & $y$ & 4 & April 17 & 11 & 15 & 10 \\
\hline SOSP & NS & $\mathrm{F}$ & 33.05 & $y$ & 4 & April 17 & 11 & 15 & 10 \\
\hline SOSP & NS & $S$ & 19 & $y$ & 4 & April 17 & 11 & 15 & 10 \\
\hline SOSP & NS & $G$ & 0 & $y$ & 4 & April 17 & 11 & 15 & 10 \\
\hline SOSP & NS & W & 0 & $y$ & 4 & April 17 & 11 & 15 & 10 \\
\hline SOSP & $\mathrm{PEI}$ & $\mathrm{F}$ & 35.23 & $y$ & 4 & April 17 & 11 & 15 & 10 \\
\hline SOSP & PEI & s & 9.5 & $y$ & 4 & April 17 & 11 & 15 & 10 \\
\hline SOSP & PEI & G & 0 & $y$ & 4 & April 17 & 11 & 15 & 10 \\
\hline SOSP & $\mathrm{PEI}$ & w & 4.96 & $y$ & 4 & April 17 & 11 & 15 & 10 \\
\hline SOSP & NL & $\mathrm{F}$ & 35.23 & $y$ & 4 & April 17 & 11 & 15 & 10 \\
\hline SOSP & $\mathrm{NL}$ & s & 9.5 & $y$ & 4 & April 17 & 11 & 15 & 10 \\
\hline SOSP & NL & G & 0 & $y$ & 4 & April 17 & 11 & 15 & 10 \\
\hline SOSP & NL & w & 4.96 & $y$ & 4 & April 17 & 11 & 15 & 10 \\
\hline SOSP & $\mathrm{NU}$ & $\mathrm{F}$ & 0 & $n$ & & & & & \\
\hline SOSP & $\mathrm{NU}$ & $S$ & 0 & $\mathrm{n}$ & & & & & \\
\hline SOSP & $\mathrm{NU}$ & G & 0 & $\mathrm{n}$ & & & & & \\
\hline SOSP & $\mathrm{NU}$ & w & 0 & $\mathrm{n}$ & & & & & \\
\hline SOSP & NT & $\mathrm{F}$ & 0 & $y$ & 4 & April 17 & 11 & 15 & 10 \\
\hline SOSP & NT & $s$ & 0 & $y$ & 4 & April 17 & 11 & 15 & 10 \\
\hline SOSP & NT & G & 0 & $y$ & 4 & April 17 & 11 & 15 & 10 \\
\hline SOSP & NT & w & 10 & $y$ & 4 & April 17 & 11 & 15 & 10 \\
\hline SOSP & $Y T$ & $\mathrm{~F}$ & 0 & $y$ & 4 & April 17 & 11 & 15 & 10 \\
\hline SOSP & YT & $\mathrm{s}$ & 0 & $y$ & 4 & April 17 & 11 & 15 & 10 \\
\hline SOSP & YT & G & 0 & $y$ & 4 & April 17 & 11 & 15 & 10 \\
\hline SOSP & YT & W & 10 & $y$ & 4 & April 17 & 11 & 15 & 10 \\
\hline CCSP & $B C$ & $\mathrm{~F}$ & 0 & $y$ & 4 & May 17 & 10 & 14 & 9 \\
\hline CCSP & $\mathrm{BC}$ & $\mathrm{s}$ & 204 & $y$ & 4 & May 17 & 10 & 14 & 9 \\
\hline
\end{tabular}




\begin{tabular}{|c|c|c|c|c|c|c|c|c|c|}
\hline Species & Prov. & $\begin{array}{c}\text { Nesting } \\
\text { Habitat }\end{array}$ & $\begin{array}{l}\text { Birds } \\
/ \mathbf{k m}^{2}\end{array}$ & $\begin{array}{c}\text { In } \\
\text { Range? }\end{array}$ & $\begin{array}{c}\text { Clutch } \\
\text { Size }\end{array}$ & $\begin{array}{c}\text { First Egg } \\
\text { Egg (Date) }\end{array}$ & $\begin{array}{c}\text { Min } \\
\text { Incubation } \\
\text { Time (d) }\end{array}$ & $\begin{array}{c}\text { Max } \\
\text { Incubation } \\
\text { Time (d) }\end{array}$ & $\begin{array}{c}\text { Mean } \\
\text { Nestling } \\
\text { Period }\end{array}$ \\
\hline CCSP & $\mathrm{BC}$ & G & 0 & $y$ & 4 & May 17 & 10 & 14 & 9 \\
\hline CCSP & $\mathrm{BC}$ & W & 0 & $y$ & 4 & May 17 & 10 & 14 & 9 \\
\hline CCSP & $A B$ & $\mathrm{~F}$ & 31.84 & $y$ & 4 & May 17 & 10 & 14 & 9 \\
\hline CCSP & $A B$ & $\mathrm{~s}$ & 25.33 & $y$ & 4 & May 17 & 10 & 14 & 9 \\
\hline CCSP & $A B$ & G & 6.96 & $y$ & 4 & May 17 & 10 & 14 & 9 \\
\hline CCSP & $A B$ & w & 39.66 & $y$ & 4 & May 17 & 10 & 14 & 9 \\
\hline CCSP & SK & $\mathrm{F}$ & 26 & $y$ & 4 & May 17 & 10 & 14 & 9 \\
\hline CCSP & SK & S & 0 & $y$ & 4 & May 17 & 10 & 14 & 9 \\
\hline CCSP & SK & $G$ & 21.42 & $y$ & 4 & May 17 & 10 & 14 & 9 \\
\hline CCSP & SK & w & 0 & $y$ & 4 & May 17 & 10 & 14 & 9 \\
\hline CCSP & $\mathrm{MB}$ & F & 8.94 & $y$ & 4 & May 17 & 10 & 14 & 9 \\
\hline CCSP & $\mathrm{MB}$ & s & 0 & $y$ & 4 & May 17 & 10 & 14 & 9 \\
\hline CCSP & $\mathrm{MB}$ & G & 32.33 & $y$ & 4 & May 17 & 10 & 14 & 9 \\
\hline CCSP & $\mathrm{MB}$ & w & 13 & $y$ & 4 & May 17 & 10 & 14 & 9 \\
\hline CCSP & ON & F & 61.2 & $y$ & 4 & May 17 & 10 & 14 & 9 \\
\hline CCSP & ON & $S$ & 0 & $y$ & 4 & May 17 & 10 & 14 & 9 \\
\hline CCSP & ON & $G$ & 16.33 & $y$ & 4 & May 17 & 10 & 14 & 9 \\
\hline CCSP & ON & w & 0 & $y$ & 4 & May 17 & 10 & 14 & 9 \\
\hline CCSP & $\mathrm{QC}$ & $\mathrm{F}$ & 61.2 & $y$ & 4 & May 17 & 10 & 14 & 9 \\
\hline CCSP & $\mathrm{QC}$ & S & 0 & $y$ & 4 & May 17 & 10 & 14 & 9 \\
\hline CCSP & $\mathrm{QC}$ & $G$ & 16.33 & $y$ & 4 & May 17 & 10 & 14 & 9 \\
\hline CCSP & $\mathrm{QC}$ & $w$ & 0 & $y$ & 4 & May 17 & 10 & 14 & 9 \\
\hline CCSP & NB & $\mathrm{F}$ & 0 & $\mathrm{n}$ & & & & & \\
\hline CCSP & $\mathrm{NB}$ & S & 0 & $\mathrm{n}$ & & & & & \\
\hline CCSP & NB & G & 0 & $\mathrm{n}$ & & & & & \\
\hline CCSP & NB & W & 0 & $\mathrm{n}$ & & & & & \\
\hline CCSP & NS & $\mathrm{F}$ & 0 & $\mathrm{n}$ & & & & & \\
\hline CCSP & NS & s & 0 & $\mathrm{n}$ & & & & & \\
\hline CCSP & NS & G & 0 & $\mathrm{n}$ & & & & & \\
\hline CCSP & NS & w & 0 & $\mathrm{n}$ & & & & & \\
\hline CCSP & $\mathrm{PE}$ & F & 0 & $\mathrm{n}$ & & & & & \\
\hline CCSP & $\mathrm{PE}$ & s & 0 & $\mathrm{n}$ & & & & & \\
\hline CCSP & $\mathrm{PE}$ & $G$ & 0 & $n$ & & & & & \\
\hline
\end{tabular}




\begin{tabular}{|c|c|c|c|c|c|c|c|c|c|}
\hline Species & Prov. & $\begin{array}{l}\text { Nesting } \\
\text { Habitat }\end{array}$ & $\begin{array}{l}\text { Birds } \\
\text { /km }^{2}\end{array}$ & $\begin{array}{c}\text { In } \\
\text { Range? }\end{array}$ & $\begin{array}{c}\text { Clutch } \\
\text { Size }\end{array}$ & $\begin{array}{c}\text { First Egg } \\
\text { Egg (Date) }\end{array}$ & $\begin{array}{c}\text { Min } \\
\text { Incubation } \\
\text { Time (d) }\end{array}$ & $\begin{array}{c}\text { Max } \\
\text { Incubation } \\
\text { Time (d) }\end{array}$ & $\begin{array}{c}\text { Mean } \\
\text { Nestling } \\
\text { Period }\end{array}$ \\
\hline CCSP & PEI & W & 0 & $\mathrm{n}$ & & & & & \\
\hline CCSP & $\mathrm{NL}$ & $\mathrm{F}$ & 0 & $\mathrm{n}$ & & & & & \\
\hline CCSP & $\mathrm{NL}$ & $\mathrm{S}$ & 0 & $\mathrm{n}$ & & & & & \\
\hline CCSP & $\mathrm{NL}$ & G & 0 & $\mathrm{n}$ & & & & & \\
\hline CCSP & $\mathrm{NL}$ & W & 0 & $\mathrm{n}$ & & & & & \\
\hline CCSP & $\mathrm{NU}$ & $\mathrm{F}$ & 0 & $\mathrm{n}$ & & & & & \\
\hline CCSP & $\mathrm{NU}$ & $\mathrm{S}$ & 0 & $\mathrm{n}$ & & & & & \\
\hline CCSP & $\mathrm{NU}$ & G & 0 & $\mathrm{n}$ & & & & & \\
\hline CCSP & $\mathrm{NU}$ & $w$ & 0 & $\mathrm{n}$ & & & & & \\
\hline CCSP & NT & $\mathrm{F}$ & 31.84 & $y$ & 4 & May 17 & 10 & 14 & 9 \\
\hline CCSP & NT & $\mathrm{S}$ & 25.33 & $y$ & 4 & May 17 & 10 & 14 & 9 \\
\hline CCSP & NT & G & 6.96 & $y$ & 4 & May 17 & 10 & 14 & 9 \\
\hline CCSP & NT & W & 39.66 & $y$ & 4 & May 17 & 10 & 14 & 9 \\
\hline CCSP & $Y T$ & $F$ & 0 & $\mathrm{n}$ & & & & & \\
\hline CCSP & $\mathrm{YT}$ & $\mathrm{S}$ & 0 & 0 & & & & & \\
\hline CCSP & $\mathrm{YT}$ & $G$ & 0 & $\mathrm{n}$ & & & & & \\
\hline CCSP & $\mathrm{YT}$ & W & 0 & $\mathrm{n}$ & & & & & \\
\hline VESP & $\mathrm{BC}$ & $\mathrm{F}$ & 14 & $y$ & 4 & April 23 & 11 & 14 & 9 \\
\hline VESP & $\mathrm{BC}$ & $\mathrm{S}$ & 0 & $y$ & 4 & April 23 & 11 & 14 & 9 \\
\hline VESP & $\mathrm{BC}$ & G & 0 & $y$ & 4 & April 23 & 11 & 14 & 9 \\
\hline VESP & $\mathrm{BC}$ & w & 0 & $y$ & 4 & April 23 & 11 & 14 & 9 \\
\hline VESP & $A B$ & $\mathrm{~F}$ & 5 & $y$ & 4 & April 23 & 11 & 14 & 9 \\
\hline VESP & $A B$ & $\mathrm{~S}$ & 0 & $y$ & 4 & April 23 & 11 & 14 & 9 \\
\hline VESP & $A B$ & G & 6 & $y$ & 4 & April 23 & 11 & 14 & 9 \\
\hline VESP & $A B$ & w & 0 & $y$ & 4 & April 23 & 11 & 14 & 9 \\
\hline VESP & SK & $\mathrm{F}$ & 0 & $y$ & 4 & April 23 & 11 & 14 & 9 \\
\hline VESP & SK & $\mathrm{S}$ & 0 & $y$ & 4 & April 23 & 11 & 14 & 9 \\
\hline VESP & SK & G & 13.95 & $y$ & 4 & April 23 & 11 & 14 & 9 \\
\hline VESP & SK & W & 0 & $y$ & 4 & April 23 & 11 & 14 & 9 \\
\hline VESP & $\mathrm{MB}$ & $F$ & 17.13 & $y$ & 4 & April 23 & 11 & 14 & 9 \\
\hline VESP & $\mathrm{MB}$ & $\mathrm{S}$ & 0 & $y$ & 4 & April 23 & 11 & 14 & 9 \\
\hline VESP & $\mathrm{MB}$ & G & 0 & $y$ & 4 & April 23 & 11 & 14 & 9 \\
\hline VESP & $\mathrm{MB}$ & w & 0 & $y$ & 4 & April 23 & 11 & 14 & 9 \\
\hline
\end{tabular}




\begin{tabular}{|c|c|c|c|c|c|c|c|c|c|}
\hline Species & Prov. & $\begin{array}{c}\text { Nesting } \\
\text { Habitat }\end{array}$ & $\begin{array}{l}\text { Birds } \\
\text { /km }^{2}\end{array}$ & $\begin{array}{c}\text { In } \\
\text { Range? }\end{array}$ & $\begin{array}{c}\text { Clutch } \\
\text { Size }\end{array}$ & $\begin{array}{c}\text { First Egg } \\
\text { Egg (Date) }\end{array}$ & $\begin{array}{c}\text { Min } \\
\text { Incubation } \\
\text { Time (d) } \\
\end{array}$ & $\begin{array}{c}\text { Max } \\
\text { Incubation } \\
\text { Time (d) } \\
\end{array}$ & $\begin{array}{c}\text { Mean } \\
\text { Nestling } \\
\text { Period } \\
\end{array}$ \\
\hline VESP & ON & $F$ & 22.26 & $y$ & 4 & April 23 & 11 & 14 & 9 \\
\hline VESP & ON & $\mathrm{S}$ & 14.4 & $y$ & 4 & April 23 & 11 & 14 & 9 \\
\hline VESP & ON & G & 22.08 & $y$ & 4 & April 23 & 11 & 14 & 9 \\
\hline VESP & ON & W & 0 & $y$ & 4 & April 23 & 11 & 14 & 9 \\
\hline VESP & QC & $\mathrm{F}$ & 10.4 & $y$ & 4 & April 23 & 11 & 14 & 9 \\
\hline VESP & QC & $\mathrm{S}$ & 0 & $y$ & 4 & April 23 & 11 & 14 & 9 \\
\hline VESP & QC & G & 12.36 & $y$ & 4 & April 23 & 11 & 14 & 9 \\
\hline VESP & $\mathrm{QC}$ & W & 0 & $y$ & 4 & April 23 & 11 & 14 & 9 \\
\hline VESP & NB & $F$ & 9 & $y$ & 4 & April 23 & 11 & 14 & 9 \\
\hline VESP & NB & $\mathrm{S}$ & 0 & $y$ & 4 & April 23 & 11 & 14 & 9 \\
\hline VESP & NB & G & 0 & $y$ & 4 & April 23 & 11 & 14 & 9 \\
\hline VESP & NB & W & 0 & $y$ & 4 & April 23 & 11 & 14 & 9 \\
\hline VESP & NS & $F$ & 9 & $y$ & 4 & April 23 & 11 & 14 & 9 \\
\hline VESP & NS & $\mathrm{S}$ & 0 & $y$ & 4 & April 23 & 11 & 14 & 9 \\
\hline VESP & NS & G & 0 & $y$ & 4 & April 23 & 11 & 14 & 9 \\
\hline VESP & NS & W & 0 & $y$ & 4 & April 23 & 11 & 14 & 9 \\
\hline VESP & PE & $F$ & 0 & $\mathrm{n}$ & & & & & \\
\hline VESP & PEI & $\mathrm{S}$ & 0 & $\mathrm{n}$ & & & & & \\
\hline VESP & PEI & G & 0 & $\mathrm{n}$ & & & & & \\
\hline VESP & PEI & W & 0 & $\mathrm{n}$ & & & & & \\
\hline VESP & NL & $F$ & 0 & $\mathrm{n}$ & & & & & \\
\hline VESP & NL & $S$ & 0 & $\mathrm{n}$ & & & & & \\
\hline VESP & NL & G & 0 & $\mathrm{n}$ & & & & & \\
\hline VESP & $\mathrm{NL}$ & w & 0 & $\mathrm{n}$ & & & & & \\
\hline VESP & NU & $F$ & 0 & $\mathrm{n}$ & & & & & \\
\hline VESP & $\mathrm{NU}$ & $\mathrm{S}$ & 0 & $\mathrm{n}$ & & & & & \\
\hline VESP & NU & G & 0 & $\mathrm{n}$ & & & & & \\
\hline VESP & NU & w & 0 & $\mathrm{n}$ & & & & & \\
\hline VESP & NT & $F$ & 0 & $\mathrm{n}$ & & & & & \\
\hline VESP & NT & $\mathrm{S}$ & 0 & $n$ & & & & & \\
\hline VESP & $\mathrm{NT}$ & G & 0 & $\mathrm{n}$ & & & & & \\
\hline VESP & NT & W & 0 & $n$ & & & & & \\
\hline VESP & $\mathrm{YT}$ & $\mathrm{F}$ & 0 & $\mathrm{n}$ & & & & & \\
\hline
\end{tabular}




\begin{tabular}{|c|c|c|c|c|c|c|c|c|c|}
\hline Species & Prov. & $\begin{array}{c}\text { Nesting } \\
\text { Habitat }\end{array}$ & $\begin{array}{l}\text { Birds } \\
\text { /km }^{2}\end{array}$ & $\begin{array}{c}\text { In } \\
\text { Range? }\end{array}$ & $\begin{array}{c}\text { Clutch } \\
\text { Size }\end{array}$ & $\begin{array}{c}\text { First Egg } \\
\text { Egg (Date) }\end{array}$ & $\begin{array}{c}\text { Min } \\
\text { Incubation } \\
\text { Time (d) } \\
\end{array}$ & $\begin{array}{c}\text { Max } \\
\text { Incubation } \\
\text { Time (d) } \\
\end{array}$ & $\begin{array}{c}\text { Mean } \\
\text { Nestling } \\
\text { Period }\end{array}$ \\
\hline VESP & $\mathrm{YT}$ & $\mathrm{S}$ & 0 & $\mathrm{n}$ & & & & & \\
\hline VESP & $\mathrm{YT}$ & G & 0 & $\mathrm{n}$ & & & & & \\
\hline VESP & $\mathrm{YT}$ & $w$ & 0 & $\mathrm{n}$ & & & & & \\
\hline MALL & $\mathrm{BC}$ & $\mathrm{F}$ & 3.1 & $y$ & 8.7 & April 2 & 24 & 31 & 1 \\
\hline MALL & $\mathrm{BC}$ & $\mathrm{S}$ & 0.1 & $y$ & 8.7 & April 2 & 24 & 31 & 1 \\
\hline MALL & $\mathrm{BC}$ & G & 1.8 & $y$ & 8.7 & April 2 & 24 & 31 & 1 \\
\hline MALL & $\mathrm{BC}$ & W & 3.4 & $y$ & 8.7 & April 2 & 24 & 31 & 1 \\
\hline MALL & $A B$ & $\mathrm{~F}$ & 20.8 & $y$ & 8.7 & April 2 & 24 & 31 & 1 \\
\hline MALL & $A B$ & $\mathrm{~S}$ & 5.5 & $y$ & 8.7 & April 2 & 24 & 31 & 1 \\
\hline MALL & $A B$ & G & 12.2 & $y$ & 8.7 & April 2 & 24 & 31 & 1 \\
\hline MALL & $A B$ & W & 22.6 & $y$ & 8.7 & April 2 & 24 & 31 & 1 \\
\hline MALL & SK & $\mathrm{F}$ & 10.2 & $y$ & 8.7 & April 2 & 24 & 31 & 1 \\
\hline MALL & SK & $\mathrm{S}$ & 2.7 & $y$ & 8.7 & April 2 & 24 & 31 & 1 \\
\hline MALL & SK & G & 6 & $y$ & 8.7 & April 2 & 24 & 31 & 1 \\
\hline MALL & SK & $w$ & 11.1 & $y$ & 8.7 & April 2 & 24 & 31 & 1 \\
\hline MALL & $\mathrm{MB}$ & $\mathrm{F}$ & 17.7 & $y$ & 8.7 & April 2 & 24 & 31 & 1 \\
\hline MALL & $\mathrm{MB}$ & $\mathrm{S}$ & 3.5 & $y$ & 8.7 & April 2 & 24 & 31 & 1 \\
\hline MALL & $\mathrm{MB}$ & G & 8 & $y$ & 8.7 & April 2 & 24 & 31 & 1 \\
\hline MALL & $\mathrm{MB}$ & $w$ & 5.1 & $y$ & 8.7 & April 2 & 24 & 31 & 1 \\
\hline MALL & ON & $\mathrm{F}$ & 18.3 & $y$ & 8.7 & April 2 & 24 & 31 & 1 \\
\hline MALL & ON & $\mathrm{S}$ & 5 & $y$ & 8.7 & April 2 & 24 & 31 & 1 \\
\hline MALL & ON & G & 8.4 & $y$ & 8.7 & April 2 & 24 & 31 & 1 \\
\hline MALL & ON & W & 22.6 & $y$ & 8.7 & April 2 & 24 & 31 & 1 \\
\hline MALL & QC & $\mathrm{F}$ & 12.8 & $y$ & 8.7 & April 2 & 24 & 31 & 1 \\
\hline MALL & QC & $\mathrm{S}$ & 3.5 & $y$ & 8.7 & April 2 & 24 & 31 & 1 \\
\hline MALL & QC & G & 5.9 & $y$ & 8.7 & April 2 & 24 & 31 & 1 \\
\hline MALL & QC & W & 15.8 & $y$ & 8.7 & April 2 & 24 & 31 & 1 \\
\hline MALL & $\mathrm{NB}$ & $\mathrm{F}$ & 13.0 & $y$ & 8.7 & April 2 & 24 & 31 & 1 \\
\hline MALL & NB & $\mathrm{S}$ & 3.5 & $y$ & 8.7 & April 2 & 24 & 31 & 1 \\
\hline MALL & NB & G & 5.9 & $y$ & 8.7 & April 2 & 24 & 31 & 1 \\
\hline MALL & $\mathrm{NB}$ & w & 16.0 & $y$ & 8.7 & April 2 & 24 & 31 & 1 \\
\hline MALL & NS & $\mathrm{F}$ & 13.0 & $y$ & 8.7 & April 2 & 24 & 31 & 1 \\
\hline MALL & NS & $\mathrm{S}$ & 3.5 & $y$ & 8.7 & April 2 & 24 & 31 & 1 \\
\hline
\end{tabular}




\begin{tabular}{|c|c|c|c|c|c|c|c|c|c|}
\hline Species & Prov. & $\begin{array}{c}\text { Nesting } \\
\text { Habitat }\end{array}$ & $\begin{array}{l}\text { Birds } \\
\text { /km }^{2}\end{array}$ & $\begin{array}{c}\text { In } \\
\text { Range? }\end{array}$ & $\begin{array}{c}\text { Clutch } \\
\text { Size }\end{array}$ & $\begin{array}{c}\text { First Egg } \\
\text { Egg (Date) }\end{array}$ & $\begin{array}{c}\text { Min } \\
\text { Incubation } \\
\text { Time (d) } \\
\end{array}$ & $\begin{array}{c}\text { Max } \\
\text { Incubation } \\
\text { Time (d) } \\
\end{array}$ & $\begin{array}{c}\text { Mean } \\
\text { Nestling } \\
\text { Period } \\
\end{array}$ \\
\hline MALL & NS & G & 5.9 & $y$ & 8.7 & April 2 & 24 & 31 & 1 \\
\hline MALL & NS & W & 16.0 & $y$ & 8.7 & April 2 & 24 & 31 & 1 \\
\hline MALL & PEl & $\mathrm{F}$ & 13.0 & $y$ & 8.7 & April 2 & 24 & 31 & 1 \\
\hline MALL & PE & $\mathrm{S}$ & 3.5 & $y$ & 8.7 & April 2 & 24 & 31 & 1 \\
\hline MALL & PEI & G & 5.9 & $y$ & 8.7 & April 2 & 24 & 31 & 1 \\
\hline MALL & PEI & W & 16.0 & $y$ & 8.7 & April 2 & 24 & 31 & 1 \\
\hline MALL & $\mathrm{NL}$ & $\mathrm{F}$ & 0 & $\mathrm{n}$ & & & & & \\
\hline MALL & $\mathrm{NL}$ & $\mathrm{S}$ & 0 & $\mathrm{n}$ & & & & & \\
\hline MALL & $\mathrm{NL}$ & G & 0 & $\mathrm{n}$ & & & & & \\
\hline MALL & $\mathrm{NL}$ & W & 0 & $\mathrm{n}$ & & & & & \\
\hline MALL & $\mathrm{NU}$ & $F$ & 0 & $\mathrm{n}$ & & & & & \\
\hline MALL & $\mathrm{NU}$ & $\mathrm{S}$ & 0 & $\mathrm{n}$ & & & & & \\
\hline MALL & $\mathrm{NU}$ & G & 0 & $\mathrm{n}$ & & & & & \\
\hline MALL & $\mathrm{NU}$ & W & 0 & $\mathrm{n}$ & & & & & \\
\hline MALL & NT & $F$ & 4.3 & $y$ & 8.7 & April 2 & 24 & 31 & 1 \\
\hline MALL & NT & $\mathrm{S}$ & 1.2 & $y$ & 8.7 & April 2 & 24 & 31 & 1 \\
\hline MALL & NT & G & 2.0 & $y$ & 8.7 & April 2 & 24 & 31 & 1 \\
\hline MALL & NT & W & 5.4 & $y$ & 8.7 & April 2 & 24 & 31 & 1 \\
\hline MALL & $\mathrm{YT}$ & $\mathrm{F}$ & 3.2 & $y$ & 8.7 & April 2 & 24 & 31 & 1 \\
\hline MALL & $\mathrm{YT}$ & $\mathrm{S}$ & 0.9 & $y$ & 8.7 & April 2 & 24 & 31 & 1 \\
\hline MALL & $\mathrm{YT}$ & G & 1.5 & $y$ & 8.7 & April 2 & 24 & 31 & 1 \\
\hline MALL & $\mathrm{YT}$ & W & 3.9 & $y$ & 8.7 & April 2 & 24 & 31 & 1 \\
\hline
\end{tabular}




\section{Road Maintenance Inputs}

The following table contains the road maintenance data used in the simulation model. Values in square brackets are estimates to represent provinces for which incomplete or no information was provided. Area estimates are based on average proportion maintained for the provinces/territories for which complete data were obtained. Timing estimates assume an even distribution of effort from June through August unless otherwise stated in the Notes field.

\begin{tabular}{|c|c|c|c|c|c|c|c|c|c|c|}
\hline \multicolumn{3}{|c|}{ Maintenance } & \multicolumn{7}{|c|}{ Timing } & \multirow[b]{2}{*}{ Notes } \\
\hline Location & Activity & Area (ha) & $\begin{array}{l}\% \\
\text { April }\end{array}$ & $\begin{array}{l}\% \\
\text { May }\end{array}$ & $\begin{array}{l}\% \\
\text { June }\end{array}$ & $\begin{array}{l}\% \\
\text { July }\end{array}$ & $\begin{array}{l}\% \\
\text { Aug }\end{array}$ & $\begin{array}{l}\% \\
\text { Sept }\end{array}$ & $\begin{array}{l}\% \\
\text { Oct }\end{array}$ & \\
\hline British Columbia & $\begin{array}{l}\text { provincial - } \\
\text { moving }\end{array}$ & 11,852 & & & & & & 50 & 50 & $\begin{array}{l}\text { provincial policy requires that works occur outside } \\
\text { breeding periods so as not to disturb nests; use a } \\
\text { nest survey protocol if works must occur within this } \\
\text { period; timing of period shifts with latitude }\end{array}$ \\
\hline British Columbia & $\begin{array}{l}\text { provincial - } \\
\text { brushing }\end{array}$ & 758 & & & & & & [50] & {$[50]$} & $\begin{array}{l}\text { includes machine and overhead brushing; timing } \\
\text { assumed to be the same as provincial moving }\end{array}$ \\
\hline British Columbia & $\begin{array}{l}\text { provincial - } \\
\text { ditching }\end{array}$ & 194 & & & & & & [50] & {$[50]$} & $\begin{array}{l}\text { this operation would affect ground-nesters (same } \\
\text { as moving); timing assumed to be the same as } \\
\text { provincial moving }\end{array}$ \\
\hline British Columbia & district & {$[3,246]$} & & & [34] & [33] & [33] & & & $\begin{array}{l}\text { no data will be coming for District roads as there is } \\
\text { no province-vide summary of their activities; could } \\
\text { try MoE for permits (would give some info about } \\
\text { timing) }\end{array}$ \\
\hline Alberta & & {$[108,685]$} & & & & & [50] & [50] & & timing estimated based on input from Don Collins \\
\hline Saskatchewan & $\begin{array}{l}\text { provincial - } \\
\text { moving }\end{array}$ & 4200 & & & 25 & 75 & & & & spring shoulder cut \\
\hline Saskatchewan & $\begin{array}{l}\text { provincial - } \\
\text { moving }\end{array}$ & 28900 & & & & 25 & 50 & 25 & & $\begin{array}{l}\text { full right-of-way cut on divided highways ( } 5900 \text { ha) } \\
\text { and shoulder cut for remainder ( } 23,000 \text { ha) }\end{array}$ \\
\hline Saskatchewan & municipal & {$[84,651]$} & & & 10 & 40 & & 50 & & $\begin{array}{l}\text { timing estimate based on input from Dale Harvey, } \\
\text { DH circulated our survey for the RMs, but input } \\
\text { wasn't received in time to be included }\end{array}$ \\
\hline
\end{tabular}




\begin{tabular}{|c|c|c|c|c|c|c|c|c|c|c|}
\hline \multicolumn{3}{|c|}{ Maintenance } & \multicolumn{7}{|c|}{ Timing } & \multirow[b]{2}{*}{ Notes } \\
\hline Location & Activity & Area (ha) & $\begin{array}{l}\% \\
\text { April }\end{array}$ & $\begin{array}{l}\% \\
\text { May }\end{array}$ & $\begin{array}{l}\% \\
\text { June }\end{array}$ & $\begin{array}{l}\% \\
\text { July }\end{array}$ & $\begin{array}{l}\% \\
\text { Aug }\end{array}$ & $\begin{array}{l}\% \\
\text { Sept }\end{array}$ & $\begin{array}{l}\% \\
\text { Oct }\end{array}$ & \\
\hline Manitoba & mowing & {$[38,623]$} & & & [34] & [33] & [33] & & & $\begin{array}{l}\text { got response too late for inclusion (April 19, 2010), } \\
\text { but the area provided in that response ( } 38,000 \text { ha) } \\
\text { was very close to the estimate we used in the } \\
\text { model; timing information was incomplete ('tvice a } \\
\text { summer on the PTH network and once in the fall on } \\
\text { the PR Network') }\end{array}$ \\
\hline Ontario & provincial & 8,671 & 1 & 3 & 15 & 23 & 21 & 17 & 11 & $\begin{array}{l}\text { provincial policy requires that maintenance not } \\
\text { disturb nests; the mitigation is not strictly a timing } \\
\text { mitigation }\end{array}$ \\
\hline Ontario & $\begin{array}{l}\text { municipal } \\
\text { placeholder }\end{array}$ & {$[40,260]$} & [1] & [10] & [21] & [21] & [19] & [20] & [8] & $\begin{array}{l}\text { survey sent Feb 3; timing based on averages for } 25 \\
\text { responses; hectares calculated by subtraction; } \\
\text { provincial environmental standards do not apply to } \\
\text { the municipalities }\end{array}$ \\
\hline Quebec & & {$[55,993]$} & & & [34] & [33] & [33] & & & no response after 2 contacts \\
\hline New Brunswick & mowing & 983 & & & 40 & 40 & 20 & & & includes shrub \& tree cutting \\
\hline New Brunswick & ditching & 72 & & & 10 & 35 & 35 & 20 & & \\
\hline New Brunswick & $\begin{array}{l}\text { NB Power } \\
\text { moving }\end{array}$ & 828 & 2 & 2 & 8 & 7 & 13 & 11 & 8 & $\begin{array}{l}\text { NB Power also mows provincial roads; about } 70 \% \\
\text { of the work occurs outside the breeding season; } \\
\text { does not include urban moving }\end{array}$ \\
\hline Nova Scotia & moving & 4,450 & & & 10 & 40 & 40 & 10 & & \\
\hline $\begin{array}{l}\text { Prince Edward } \\
\text { Island }\end{array}$ & brushing & 465 & 0 & 0 & 16 & 28 & 28 & 28 & 0 & $\begin{array}{l}\text { brushing starts in latter half of June, and effort is } \\
\text { evenly distributed over the } 14 \text { weeks to the end of } \\
\text { September; the area brushed is in addition to the } \\
\text { area mowed }\end{array}$ \\
\hline $\begin{array}{l}\text { Prince Edward } \\
\text { Island }\end{array}$ & mowing & 3075 & 0 & 0 & 40 & 0 & 20 & 0 & 40 & $\begin{array}{l}1025 \text { ha gets cut in latter half of June, and then it all } \\
\text { gets cut again in mid-August, and then it all gets cut } \\
\text { again in late September or early October for snow } \\
\text { management; the first cut will almost certainly result } \\
\text { in take, and the third will almost certainly NOT } \\
\text { result in take; the second cut will probably result in } \\
\text { some take, but only for birds that like short } \\
\text { vegetation; have used timing to "discount" the } \\
\text { amount of take on the second moving }\end{array}$ \\
\hline
\end{tabular}




\begin{tabular}{|c|c|c|c|c|c|c|c|c|c|c|}
\hline \multicolumn{3}{|c|}{ Maintenance } & \multicolumn{7}{|c|}{ Timing } & \multirow[b]{2}{*}{ Notes } \\
\hline Location & Activity & Area (ha) & $\begin{array}{l}\% \\
\text { April }\end{array}$ & $\begin{array}{l}\% \\
\text { May }\end{array}$ & $\begin{array}{l}\% \\
\text { June }\end{array}$ & $\begin{array}{l}\% \\
\text { July }\end{array}$ & $\begin{array}{l}\% \\
\text { Aug }\end{array}$ & $\begin{array}{l}\% \\
\text { Sept }\end{array}$ & $\begin{array}{l}\% \\
\text { Oct }\end{array}$ & \\
\hline $\begin{array}{l}\text { Prince Edward } \\
\text { Island }\end{array}$ & tree trimming & 15 & 0 & 0 & 30 & 0 & 0 & 35 & 35 & $\begin{array}{l}\text { tree trimming is in addition to area brushed; usually } \\
\text { starts in June and they may skip the summer and } \\
\text { go back to it in the fall; average } 10-15 \mathrm{~km} \text { per year }\end{array}$ \\
\hline $\begin{array}{l}\text { Newfoundland \& } \\
\text { Labrador }\end{array}$ & mowing & 0 & 0 & 0 & 0 & 0 & 0 & 0 & 0 & roadsides are not mowed \\
\hline $\begin{array}{l}\text { Newfoundland \& } \\
\text { Labrador }\end{array}$ & brushing & 220 & 0 & 0 & 0 & 0 & 0 & 0 & 50 & $\begin{array}{l}\text { averaged over the last } 4 \text { years; occurs from late } \\
\text { October through December }\end{array}$ \\
\hline Nunavut & & [199] & & & [34] & [33] & [33] & & & no response after 2 contacts \\
\hline $\begin{array}{l}\text { Northwest } \\
\text { Territories }\end{array}$ & mowing & 2750 & 0 & 0 & 15 & 30 & 40 & 15 & 0 & $\begin{array}{l}\text { includes brushing, clearing rights-of-way for } \\
\text { highways, airports and some community roads }\end{array}$ \\
\hline $\begin{array}{l}\text { Northwest } \\
\text { Territories }\end{array}$ & mowing & 100 & 10 & 10 & 20 & 25 & 25 & 10 & 0 & $\begin{array}{l}\text { includes ditch cleaning, off-takes clearing and } \\
\text { culvert repair/replacement }\end{array}$ \\
\hline Yukon & $\begin{array}{l}\text { mowing \& } \\
\text { brushing }\end{array}$ & 1220 & 0 & 0 & 0 & 10 & 45 & 35 & 10 & $\begin{array}{l}\text { percentages are an estimate based on receipt of } \\
\text { invoices }\end{array}$ \\
\hline
\end{tabular}




\title{
Avian Incidental Take due to Mining Operations in Canada
}

\author{
FINAL REPORT
}

Prepared by

ArborVitae Environmental Services Ltd.

Prepared for

Environment Canada

Western Arctic Unit, Yellowknife

December 17, 2010 


\section{Acknowledgements}

The author of this project has benefited from discussions with many people in government and industry. I would like to thank the following for their contributions:

- Moss Giasson, Diane Howe, Angeline Tillmans, Steve Cranbrook, (British Columbia provincial government)

- Gavin Direm (AME BC)

- Teri Muhlbeier (Alberta Sand and Gravel Assn)

- Tim Moulding, Ann Riemer, Gordon Gray, Mike Balfour, Wes Kotyck, Rick Stilling (Saskatchewan government)

- Leslie Hymers (Ontario Mining Assocation)

- Brian Polhill (Ontario government)

- Raymond Bullac (Quebec government)

- Line Rochefort (Laval)

- Michael Cadman, Jean-Pierre Savard (Environment Canada)

Suggested Citation: Williams, Jeremy. 2010. Avian Incidental Take due to Mining Operations in Canada. Report Prepared by ArborVitae Environmental Services Ltd. for Environment Canada, Western Arctic Unit, Yellowknife. $32 \mathrm{pp}$. 


\section{Table of Contents}

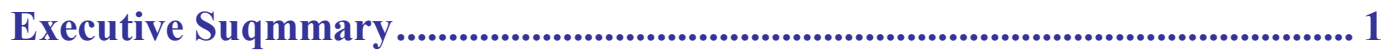

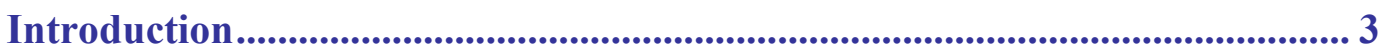

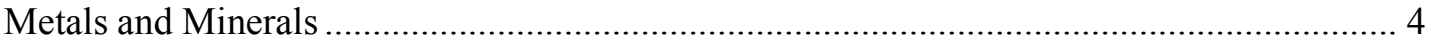

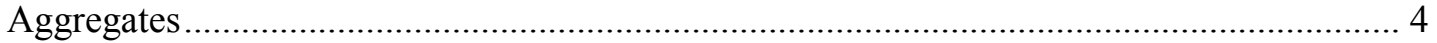

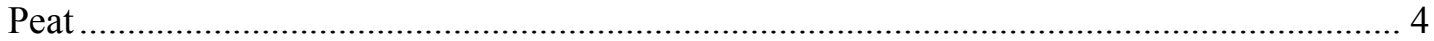

Basic Methodology and Analysis .......................................................................... 6

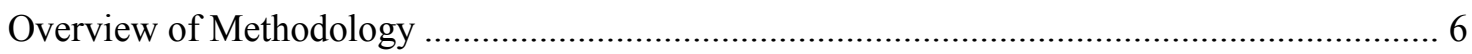

Incidental Take Associated with Metals \& Minerals.......................................... 7

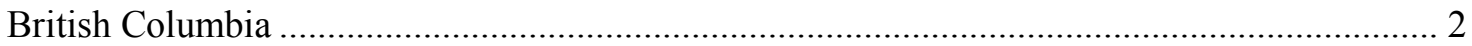

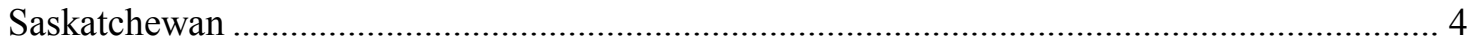

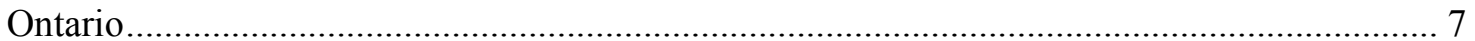

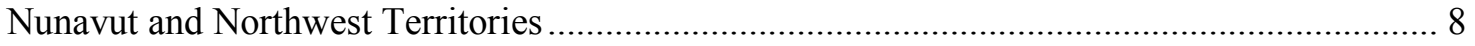

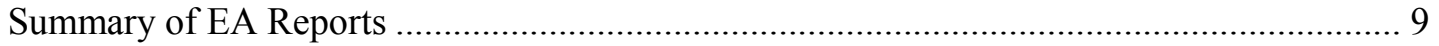

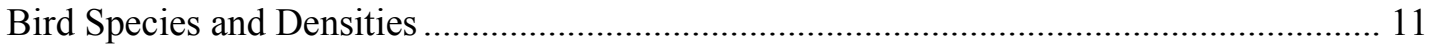

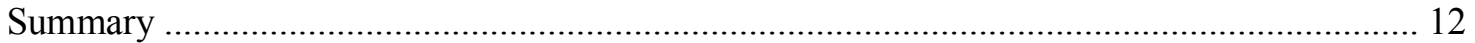

Incidental Take Associated with Aggregate Pits............................................. 14

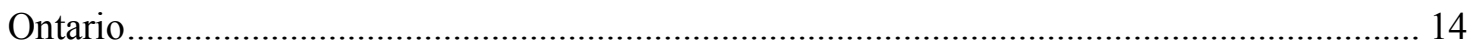

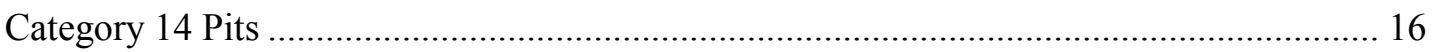

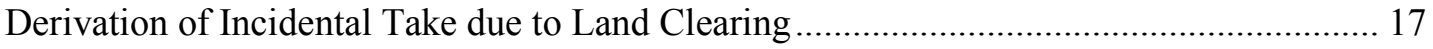

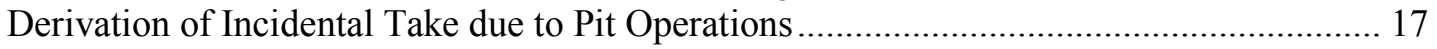

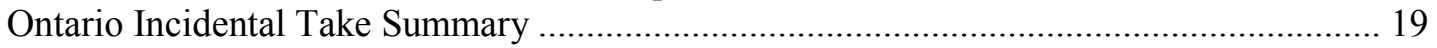

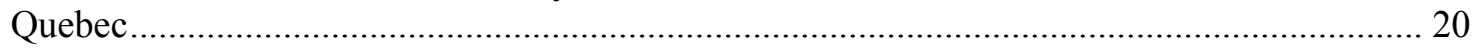

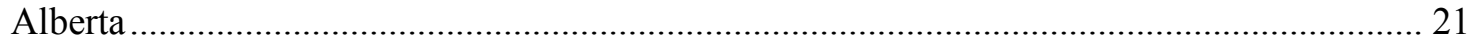

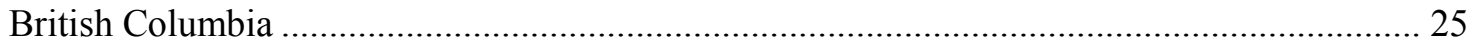

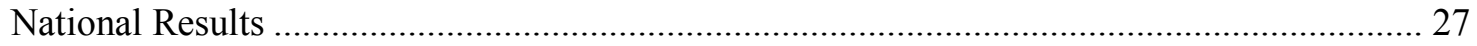

Incidental Take Associated with Commercial Peat Harvesting ..................... 28

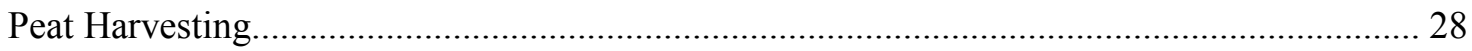

Literature Cited ........................................................................................... 30 


\section{Executive Summary}

The incidental take of birds covered by the Migratory Birds Convention Act was estimated for activities undertaken in the mining sector. The scope of the analysis covered most of common mining activities, with the exception of oil sands extraction. The assessment looked at metals and mineral extraction, aggregate mining and quarrying, and peat extraction.

There was virtually no IT identified as being associated with peat extraction because the main activity that has the potential to cause IT - the stripping of the vegetation layer at the beginning of operations - is often done in winter, while the removal of the upper peat layer to expose the mineable peat often takes place in late summer or fall. Once a bog is in production, it is inhospitable for birds and so there is no IT involved.

In the case of metals and mineral extraction, there is a meaningful level of IT. There was a considerable level of uncertainty in the estimates of IT associated with mining, so that high and low estimates were generated. The range of these estimates is very likely to include the actual value, in the opinion of the author. The variables with the greatest uncertainty were:

- The proportion of land clearing that takes place during nesting season; and

- The amount of land cleared annually, which is primarily associated with the expansion of open pit facilities.

There was a considerable variation in the estimate of the mine footprints between B.C.

Saskatchewan and Ontario, however the author has considerable confidence in the numbers because all values were derived from data in the case of B.C. and from extensive discussions with government and industry staff in the sector, in the case of Saskatchewan and Ontario. Thus, if the amount of IT is near the upper end of the metal mining estimate, then pits and quarries operations produce roughly the same level of IT as metal mining does. However, the low end estimate of IT associated with metals mining is approximately $15 \%$ of the estimated IT associated with pits and quarries.

In the case of pits and quarries, two sources of IT were identified - the clearing of the land to establish or enlarge a pit (vegetation removal and overburden stripping) and the extraction of the material that can cause IT to killdeer and bank swallows. There were some data available which were used to base the estimates of bank swallow mortality on, however the author does not have a great deal of confidence in these data since they are based on a relatively small sample size. Key variables which have a significant amount of uncertainty associated with them include:

- The average number of nests in a colony;

- The number of colonies per pit;

- The percentage of pits that have operations and mortality in them in any given year; and

- Rates of colony destruction.

High and low estimates were not developed because there were data available, however as mentioned above, the author is not highly confident of the precision of the values however he believes they are within reasonable range of true values. 


\begin{tabular}{|l|r|r|r|r|r|}
\hline & $\begin{array}{c}\text { Pits \& } \\
\text { Quarries }\end{array}$ & $\begin{array}{c}\text { High } \\
\text { Est } \\
\text { Mining }\end{array}$ & $\begin{array}{c}\text { High } \\
\text { SUM }\end{array}$ & $\begin{array}{c}\text { Low Est } \\
\text { Mining }\end{array}$ & $\begin{array}{c}\text { Low } \\
\text { SUM }\end{array}$ \\
\hline $\begin{array}{l}\text { Nfld, NS, NB, Que \& } \\
\text { MB }\end{array}$ & 33887 & 59221 & 93108 & 7896 & 41783 \\
\hline Ontario & 49876 & 25974 & 75850 & 3463 & 53339 \\
\hline Saskatchewan & 993 & 745 & 1738 & 186 & 1179 \\
\hline Alberta & 7169 & 887 & 8056 & 221 & 7390 \\
\hline British Columbia & 33604 & 32453 & 66057 & 6722 & 40326 \\
\hline Territories & 0 & 488 & 488 & 165 & 165 \\
\hline SUM & 125529 & 119768 & 245297 & 18653 & 144182 \\
\hline
\end{tabular}

Table 1. Estimated Incidental Take in Canada due to Aggregate Pit Operations. 


\section{Introduction}

The inadvertent destruction of birds and/or their nests and young occurs in Canada during otherwise legitimate operations in a variety of sectors, including forestry, mining, agriculture, electrical generation and transmission, fishing, roadside maintenance and road construction. Such "incidental take" is an important factor in bird conservation and management, and Environment Canada has identified a need to better understand the magnitude and significance of the issue.

This report documents a research and modeling effort to estimate the magnitude of avian mortality due to activities associated with mining across Canada, for species that breed in Canada and are covered under the Migratory Birds Convention Act (MBCA). The MBCA does not protect raptors, corvids, blackbirds, or gallinaceous birds, among others not specifically listed in the Convention.

In consultation with the Canadian Wildlife Service (CWS) project authority, the scope for this project has been defined as coverage of mining or extracting from the earth the following materials:

- metallic ore, including precious and industrial metals;

- coal and uranium;

- potash;

- diamonds;

- clay, sand, gravel and aggregates; and

- miscellaneous materials such as salt, gypsum and peat.

Oil sands mining is excluded from this study (it is part of another study in the same series).

The activities associated with mining begin with prospecting, or early stage exploration. This is often done using airborne detectors, however there are still elements of ground-based work involved, especially once a localized area has been identified as being of interest. Once a potential deposit of interest has been identified, the land is claimed or staked and further work is undertaken to determine the location, extent and grade of the deposit. There are many factors that influence the economic attractiveness of a project, and the activities associated with resource delineation and the development of a mine plan may easily take five years or more. If the deposit appears to be physically and economically feasible to extract, and a major facility is required, an environmental assessment (EA) will be undertaken. An EA is also generally required for the major expansion of an existing mine. Once the EA has been successfully completed, the facility is constructed. The nature of the facility depends on the location, characteristics of the deposit and mode of extraction, material being extracted, and such, however access roads and power lines are often constructed, as well as the physical mine site, tailings ponds and on-site processing facilities. The mine footprint is strongly influenced by whether the operation is an open pit mine or whether it is constructed underground, or is a combination of the two approaches.

Incidental take may be associated with all stages of mining, and it will most often be associated with land clearing for roads, drill site, resource sampling, and the construction of the mine site and related infrastructure. We do not here consider incidental caused by collisions with mining vehicles or mine infrastructure; those sources of IT would be considered to fall into other components of IT which have been studied separately. 
In the case of aggregate pits (note there are pits for the production of sand, gravel, and aggregate, and quarries for stone - these shall collectively be referred to as aggregate pits), there is also exploratory activity but it is generally more limited and does not involve as much land clearing as in the case of metals and minerals. When a pit or quarry is developed, there is also often no environmental assessment unless it is especially large or in an especially sensitive setting. Aggregate pits are attractive nesting sites for killdeer and bank swallows also may nest in the steep sites of a pit. Therefore, the major activities that could produce incidental take associated with pits and quarries are the land clearing for pit establishment and enlargement, and activities associated with the operation of an existing pit where bank swallows have nested in the face of the pit side and/or killdeer have nested in the pit itself.

In summary, the operations considered in this analysis are:

Metals and Minerals

Activities:

Activities:

Peat

Activities:
Exploration, deposit appraisal, mine and mine-related infrastructure construction, and mine operation/expansion. Mine-related infrastructure may include access roads, power lines and dams, space for the facilities, and other operations (e.g. a small lake was moved to provide access to the Ekati mine in Northwest Territories). Includes coal, potash, uranium and diamonds as well as base metals and precious metals. Most of the coal, potash, uranium and diamond mines are open pit mines, but there are some that are underground (e.g. Snap Lake - diamonds; Cigar Lake - uranium) a. Similarly, just more than half of the existing and under development metal mines in B.C. are open pit; the remainder are below ground.

The activities covered include the removal of the surface vegetation and overburden to start a pit or expand an existing pit and the annual operations in existing pits.

The primary activities of interest are the preparation of the peat bogs for extraction - operations include felling any trees, creating a series of drainage ditches to dry out the peat deposit, and the stripping of the surface vegetation. In recent years, operators have begun to mix the surface vegetation into the peat deposit (excluding trees) rather than remove it. Once the peat deposit has been prepared, the peat is removed gradually over a period of years.

The mining industry, including harvesters of peat and companies that remove sand, stone, and aggregate, are required to rehabilitate spent mines, pits and quarries. There is considerable scrutiny to ensure that rehabilitation is undertaken well, and this will restore habitat to the mine site once the rehabilitation work is established. While many companies do a good job at this, this does not offset the calculation of incidental take in this study, which is concerned with the mortality caused by initial and on-going operations. 
Mining operations often take place in very remote areas and mining is often seen as a means for developing or opening up an area. As indicated above, mines have substantial power needs, as well as a need for road and sometimes rail access. As a result, governments may undertake major regional infrastructure projects to support mining. There are currently several such projects being undertaken in Canada by provincial and territorial governments that are designed to provide access and power to remote areas that are known to have major mineral reserves (Canadian Intergovernmental Working Group on the Mining Industry, 2010):

- The extension of Highway 167 in northern Quebec: in its March 2009 budget, the Quebec government announced the $\$ 130$ million extension of Highway 167 from Chibougamau to the Otish Mountains. The extended highway will facilitate access to projects such as Renard (diamonds), Matoush (uranium), and Macleod Lake (copper-molybdenum).

- The Northwest Transmission Line (NTL) in northwestern British Columbia: on September 16, 2009, the federal government announced a $\$ 130$ million commitment to help build the 335-km NTL. Part of the remaining \$274 million, from a total cost of \$404 million, would come from private industry, but the Government of British Columbia, which has committed to build the NTL, would bear the largest costs with a contribution that could reach a reported $\$ 250$ million. Upon completion, the NTL would improve the economics of a number of significant mining projects in northwestern British Columbia, including Galore Creek, Kerr-Sulphurets-Mitchell, and Schaft Creek.

- The Bathurst Inlet Port and Road (BIPR) project in Nunavut: this proposed deep-water port and permanent all-weather road would foster mineral exploration and production in the Kitikmeot region of Nunavut by reducing transportation costs associated with projects in this area and by lengthening the trucking season. A 50-50 joint venture between Kitikmeot Corporation and Nuna Logistics, the BIPR would be financed through a public-private partnership. Its Environmental Impact Statement is currently on hold with the Nunavut Impact Review Board. Examples of projects that could benefit from the BIPR include Hope Bay (gold); Izok Lake, NICO, Hackett River, and High Lake (all base metals); Gahcho Kue (diamonds); and Thor Lake (REE).

Because these projects are not being undertaken solely to support mining, the incidental take associated with them is considered to come under the powerline and road construction and maintenance categories, and not mining. 


\section{Basic Methodology and Analysis}

\section{OVERVIEW OF METHODOLOGY}

The most widespread cause of incidental take associated with mining is the clearing of land. Because land clearing is essentially the same no matter what metal, mineral, or stone is to be mined, the same basic approach to the calculation of incidental take was followed for all components of mining. In addition, for the activities associated with aggregate pits, there were additional calculations of the within-pit mortality of killdeer and bank swallows.

This section provides an overview of the methodology for calculating the incidental take associated with land clearing for the development or expansion of mines, pits and quarries.

The key data required are:

- Area cleared per year, by broad habitat type and region

- $\%$ of area cleared during nesting season

- Density of nesting birds and average clutch size

- $\%$ of birds and eggs killed per ha of land cleared during nesting season

Mining activities are undertaken in each province and territory, but the activity is not evenly distributed across the country. In metal and mineral mining, five provinces and territories are dominant. Therefore for each mining sub-sector, detailed analyses were undertaken for the most important jurisdictions, and the results extrapolated to other provinces and territories based on the level of activity in those jurisdictions.

Bird IT mortality was calculated for two general habitat types - grassland/scrubland and forest. No data were found specifying the amount of area cleared by habitat type - while a reasonable estimate of area cleared could be developed from available data, the author used a combination of his knowledge of the country and discussion with experts to estimate the proportion of each habitat type cleared. In addition, mining for metals and diamonds, and related exploration, is being undertaken in Nunavut and Northwest Territories, and a "sub-arctic habitat" IT figure was derived for this segment of the mining sector.

In the case of metal and mineral mines, the basic calculation was to estimate the number of new mines /major mine expansions per province or territory per year, the associated footprint, and the timing of clearing and type of habitat involved. Information on new mine types and locations was readily available, and information on footprint size was obtained from government and industry personnel, and from literature in some cases.

The nature of the mining process is important from the perspective of incidental take, since the area disturbed to create and operate an open pit mine is more extensive than the area requirements for an underground mine. Moreover, in the classic strip-mining process, a strip of land is cleared, the resource excavated, and the process is repeated on successive strips of land. In contrast, the footprint for the latter type of mine is essentially established when the mine starts up, unless a future expansion takes place. Frequently mines start out as open pit operations and then convert to underground mines once the excavation depth exceeds what is feasible to be extracted from an open pit. 
Estimating the incidental take associated with exploration was perhaps the most difficult task, in that there was no systematic information available on the amount of area disturbed. Where a deposit is found and there is considerable resource delineation activity, much of this will fall on what turns out to be the mine footprint, should a mine be built. Seasonality also played a major role in the assessment of impacts, as well as discussions with those in the sector regarding the introduction of low impact (and also low cost) methods of exploration.

All aggregate pits, whether they be on public or private land, must be permitted. Most jurisdictions tabulate, or have access to, the area covered by the leases, licences and permits. However, the area covered under any individual authorization is generally larger than the area of the actual pit, and there was much less information available regarding actual pit area. The Ontario Aggregate Resources Corporation (TOARC) was the only source that was located of annual new pit area. In other jurisdictions, an estimate of area cleared per annum was made based on the term of the licence. Some licence types were only valid for pits of a defined size, and where this was so, this information was considered in estimating annual new disturbance area. In general, there was less information about pits on private land, which is unfortunate since that is where the majority of pits are located.

The seasonality of operations is critical - if most land clearing takes place outside of the nesting season, there will be no incidental take, or certainly negligible amounts. There were no published data on this, and it was through discussions with industry and government staff that an estimate was determined. Reasonable data were available regarding average clutch size however little information was available regarding what proportion of the eggs, young and adult birds are killed when land is cleared during nesting season.

There were also some intangible factors that were taken into account as well. Pits on public land are more closely regulated, and can be inspected by government staff from time to time. This created the impression that operators were less likely to cause incidental take of bank swallows in pits on public land. In addition, the Ontario industry members that were contacted were all aware of the Migratory Bird Convention Act and stated that there were best practices to not operate where there were nesting colonies. Provincial government staff were also aware of the MBCA. This created the impression that at least some operators to try to avoid causing incidental take. In contrast, pit operators on private land in Alberta operate with fair less constraint. The Alberta Sand and Gravel Association was unable to collect basic operational information from its own members. In addition, none of the interviewees in the province mentioned the MBCA. This created the strong impression that there was considerable IT associated with the operation of existing pits, and a factor was added to account for this.

The within-pit incidental take of killdeer and bank swallows was based on the number of pit licences and permits (as a proxy for the number of pits), an estimate of the number of nests (killdeer) and colonies (bank swallows) in each pit and the number that are destroyed by regular extraction operations.

\section{Incidental Take Associated with Metals \& Minerals}

This section covers much of what is considered to be traditional mining, namely the extraction of precious metals such gold and silver, and base metals such as copper, zinc, and nickel. Also 
included are coal, uranium, potash, and diamonds. There are numerous mines in Canada, and from 2004 onwards there was a tremendous amount of exploration that ended abruptly in 2008 when commodity prices declined sharply, credit became difficult to find, and the world economy went into recession. However, from the initial discovery of a potentially mineable resource, it will take a minimum of 4-6 years to develop an understanding of the nature and grade of the deposit, to formulate a mine plan and assess of the economics of the project. If the project appears to be able to generate a favourable rate of return, permitting may take several more years, since new mines and major mine expansions require environmental assessments. If a mine passes through the EA process, another 2-3 years are generally required to construct the facilities and bring it into production. Thus, mine development is a long-term undertaking, and while recessions can and do derail the process in some cases (especially when the mine developer is a small company), in most instances, once the resource has reached the permitting stage, mine development will proceed regardless of economic fluctuations.

To give the reader an idea of the number of mines, Figure 1 is a map of active mines and development projects in British Columbia, which has traditionally been among the top three mining provinces. As of 2008, there were 10 active metal mines, 9 coal mines and 35 industrial material mines in the province, with 3 metal mines and 1 coal project in an advanced stage. Another 10 metal mines, 7 coal mines and two industrial mineral quarries were in the EA process, and there were 118 major exploration projects. The more numerous early stage exploration projects are not shown.

The ratio of active mines to mines in development is low in British Columbia, since the province experienced a hiatus of exploration and development in response to the provincial government's 1997 decision to bar the development of a promising large mine in northwest B.C. due to its location in an environmentally sensitive zone. As a result, since 1997 there has been only one moderately sized molybdenum mine opened in B.C. (Chalmers, pers. comm.).

While many of the mines are underground, a surprisingly large number are open pit mines. In part this reflects the more appealing economics associated with this approach, including a more rapid mine start-up once all of the permits have been obtained. A review of the ten operating metal mines in British Columbia indicates that 6 are open pit mines, yielding minerals ranging from gold to copper to molybdenum (Source 2009 Map of Major Mining Projects in BC). Of four mines in advanced development, three are open pit projects (see Table 5).

Many of the non-metallic resources are extracted from open pit mines. Of the 9 active coal mines in the province, only one, the Quinsam Coal Mine near Campbell River, British Columbia, is an underground mine. In fact, it is Canada's last operating underground coal mine - all of the others are open pit/strip mines. Many of the uranium and diamond mines are open pit as well.

Many metal mines are also of the open pit variety, or begin with an open pit process before transitioning to an underground operation once the upper layers of the resource have been removed. 
Figure 1. Insert map BC major projects maps OF 2009.pdf 
There is some level of mining in almost all provinces and territories, with Prince Edward Island being the exception. Figure 2 below shows the distribution of mining expenditures by province and territory in 2008. Ontario attracted the greatest total expenditure, with $20 \%$ of the total. Saskatchewan and Quebec were close behind, attracting 17.7 and $16.6 \%$ of total expenditures, respectively. Saskatchewan attracted the highest share of mine development expenditures, at $20 \%$. British Columbia attracted $13.6 \%$ of total expenditures, and the distribution of spending was notable in that $30 \%$ of all deposit appraisal expenditures were in that province. This suggests that B.C. will be the location of a greater proportion of future incidental take than its share of overall expenditures would indicate. Nunavut and Northwest Territories have attracted significant capital in recent years, and they account for 8.7 and $10.1 \%$ of all expenditures. There is a significant gap between the expenditures in the next most active provinces - Alberta, Manitoba and Newfoundland and Labrador each attracted between 2-4\% of total spending.

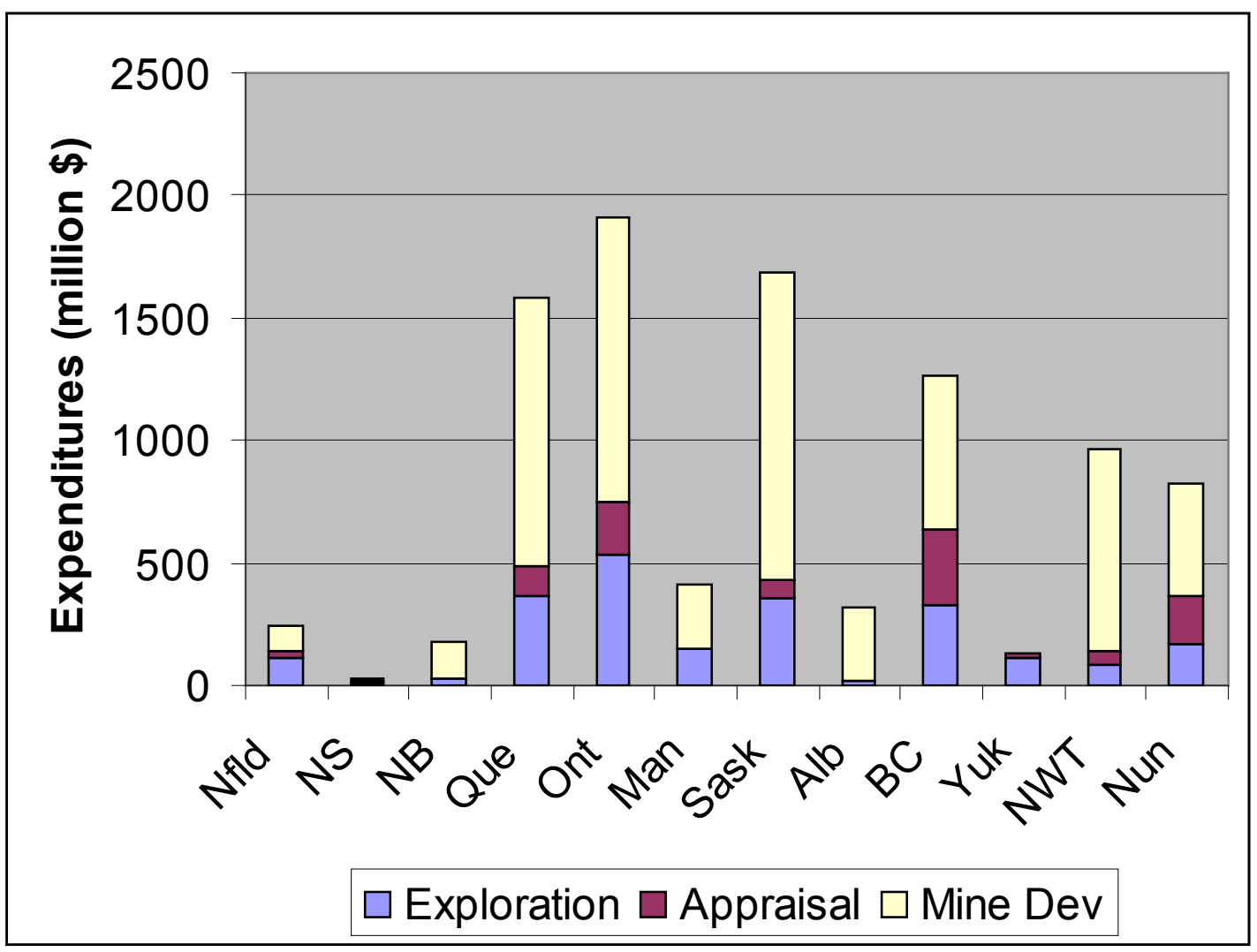

Figure 2. 2008 Mining expenditures, by major activity: Exploration, Depos it Appraisal, and Mine Complex Development (million \$).

Source: The Mining Association of Canada. 2009. Facts and Figures 2009.

To optimize resource use in this project, the more detailed investigation of incidental take will focus on Ontario, Saskatchewan, British Columbia and Nunavut \& Northwest Territories. The results of the analysis in Ontario will be pro-rated to estimate incidental take in Quebec, Manitoba and eastern Canada on the basis of expenditure levels, all of the provinces from Manitoba east are expected to have very similar rates of IT of similar species. The same logic is used to estimate Alberta's IT based on the estimate for Saskatchewan, and expenditures will be used to pro-rate incidental take estimates from Northwest Territories and Nunavut to apply to Yukon Territory. 
The next section of the report will discuss the extent and timing of the operations that could potentially lead to incidental take. In general, there was little documentation that was obtained that provided specific information about the season in which land clearing was undertaken. However, interviews with various industry members, and what documentation was found, all pointed to winter as being when the majority of the land clearing work was undertaken, whether it was for exploration activities or for clearing land for mine development (including for associated infrastructure).

\section{B RITISH COLUMBIA}

British Columbia has historically been a major mining province but experienced a decade of minimal activity in the wake of the decision to halt a proposed mine in the Tatshenshini region. However, with changes in the provincial government and a boom in commodities, exploration in B.C. has increased significantly in (See for example Figure 1). Currently, there are 5 new mines expected to start up in the next 4-5 years (See Table 2), and these are highly likely to proceed to operating mines unless there is another economic downturn. This translates into an average of 11.25 new mines opening in each of the next four or five years. Most of these are open pit mines.

\begin{tabular}{|l|l|l|l|l|}
\hline \multicolumn{1}{|c|}{ Mine } & \multicolumn{1}{|c|}{ Company } & \multicolumn{1}{c|}{ Mineral } & \multicolumn{1}{c|}{ Mine Type } & \multicolumn{1}{c|}{ BCR } \\
\hline $\begin{array}{l}\text { Tulsequah } \\
\text { Chief }\end{array}$ & Redfern Resources & $\begin{array}{l}\text { Gold-Zinc-Lead- } \\
\text { Copper }\end{array}$ & Open Pit & $\begin{array}{l}\text { Northwestern } \\
\text { interior forest }\end{array}$ \\
\hline Ruby Creek & $\begin{array}{l}\text { Adanac Molybdenum } \\
\text { Corp }\end{array}$ & Molybdenum & Open Pit & $\begin{array}{l}\text { Northwestern } \\
\text { interior forest }\end{array}$ \\
\hline New Afton & New Gold & Copper-Gold & Underground & Great Basin \\
\hline $\begin{array}{l}\text { Prosperity } \\
\text { Mine }\end{array}$ & Taseko Mines Ltd & Copper-Gold & Open Pit & $\begin{array}{l}\text { Northern } \\
\text { Rockies }\end{array}$ \\
\hline Willow Creek & Western Coal & Coal & Open pit & $\begin{array}{l}\text { Northwestern } \\
\text { interior forest }\end{array}$ \\
\hline
\end{tabular}

Table 2. B.C. Mines in the late stages of development and expected to open within the next 5 years.

Data provided by the B.C. Ministry of Energy, Mines, and Petroleum Resources show that the total disturbed area associated with coal mines is 21,645 ha and the disturbed area associated with metal and mineral mines is $24,507 \mathrm{ha}$. The disturbed areas include roads, tailings ponds, waste piles, and pits, as well as other less area-intensive uses. Some rehabilitated area that has not yet been taken out of the database is also included. There are about 50 metal mines that report approximately 25 operating and 25 closed (D. Howe, British Columbia Ministry of Energy, Mines and Petroleum Resources; pers. comm.). About 20 coal mines report -10 active and 10 closed or in start up.

These data suggest that the average footprint of a metal mine is $0.5 \mathrm{sq} \mathrm{km}$, and for a coal mine it is $1.1 \mathrm{sq} \mathrm{km}$. There is considerable variation in mine size; the Kemess and Highland Valley mines are huge, each having a distance of $7 \mathrm{~km}$ between the mine site and tailings. (D. Howe, British Columbia Ministry of Energy, Mines and Petroleum Resources; pers. comm.). Based on these data, the author has assumed that the full footprint of the underground mine is created immediately, whereas an open pit mine starts with a $0.5 \mathrm{sq} \mathrm{km}$. The four new open pit mines were assumed to come on stream at a rate of one per year for the next four years, and the underground mine was assumed to come on stream in 2011. All but one of the existing coal mines are open pit. Open pit mines expand their footprint over time, however the consultant did not find any information that provided a basis for estimating the average annual rate of expansion. Therefore, a high rate of $0.25 \mathrm{sq} \mathrm{km} / \mathrm{year}$ and a low rate of $0.1 \mathrm{sq} . \mathrm{km} / \mathrm{yr}$ were used. The author 
did not have data regarding the number of existing metal mines that are open pit versus underground, but estimated that the proportion ranged from $25-50 \%$.

The author used the map of major projects to identify the numbers of mines in each BCR. Hobson et al. (2009) provided forest bird density information for each BCR. In the northern Rockies and northwest interior BCRs, $100 \%$ of the area cleared was assumed to be forest, while in the Great Basin BCR, $50 \%$ of the area cleared was estimated to be forest and the remainder grassland.

It is reasonable to expect that a certain amount of the clearing and development work will be done out of nesting season, but an estimate of the timing of operations was not obtained from industry sources. The author selected a range of $10-30 \%$ of the cleared area as a reasonable estimate, largely based on the expectation that the seasonality dynamics are similar to those described in Saskatchewan (See next section). Table 3 shows the estimated range of incidental take due to mine development in B.C., which totaled 32,453 eggs and young birds. The data elements shown in the table are for the upper estimate. The lower IT estimate, which totaled 6,722 eggs and young birds, was calculated based on the estimate that $25 \%$ of the existing metal mines were open pit (underground mines were assumed not to expand their footprint on a predictable basis), that the annual rate of expansion was 100 ha/year and on the basis that only $10 \%$ of the clearing took place during nesting season.

\begin{tabular}{|l|r|r|r|}
\hline Parameter $\backslash$ BCR & \multicolumn{1}{c|}{$\begin{array}{c}\text { Great } \\
\text { Basin }\end{array}$} & \multicolumn{1}{c|}{$\begin{array}{c}\text { North } \\
\text { Rockies }\end{array}$} & \multicolumn{1}{c|}{$\begin{array}{c}\text { NW } \\
\text { Interior }\end{array}$} \\
\hline \# new mines in 2011 & 1 & 1 & 500 \\
\hline Avg mine foot print (ha) & 500 & 500 & 12 \\
\hline \# existing metal mines & 5 & 8 & 50 \\
\hline \% open pit & 50 & 50 & 250 \\
\hline $\begin{array}{l}\text { avg annual expansion (ha/open pit } \\
\text { mine) }\end{array}$ & 250 & 250 & 3 \\
\hline \# existing coal mines & 0 & 5 & 100 \\
\hline \% open pit & 100 & 100 & 250 \\
\hline $\begin{array}{l}\text { avg annual expansion (ha/open pit } \\
\text { mine) }\end{array}$ & 250 & 250 & 2250 \\
\hline Mine area cleared (ha/yr) & 1125 & 2750 & 100 \\
\hline \% forest cleared & 50 & 100 & 0 \\
\hline \% grassland cleared & 50 & 0 & 30 \\
\hline \% area cleared in nesting season & 30 & 30 & 3.7 \\
\hline forest bird density (pairs/ha) & 7.72 & 6.56 & 0.2 \\
\hline Grassland bird density (pairs/ha) & 0.2 & 0.2 & 3.51 \\
\hline clutch size (eggs/nest) & 3.51 & 3.51 & 100 \\
\hline \% destroyed during clearing & 100 & 100 & 8766 \\
\hline IT estimate (upper) & 4691 & 18996 & 1364 \\
\hline IT estimate (lower) & 869 & 4490 & \\
\hline
\end{tabular}

Table 3. Es stimated Range of Incidental Take due to Mining in B ritish Columbia. 
For this segment of the mining sector, there is assumed to be very little in-pit mortality of bank swallows and killdeer, primarily because the mines operate 365 days per year and there is no quiet time during which the birds would begin to nest. In addition, the pit walls are more likely to be rocky which would limit bank swallow nesting opportunities.

The consultant was unable to obtain any information relating to area disturbed during exploration, and there is surely some and surely some of the disturbance occurs during nesting season and causes incidental take. However there was very little information available upon which to make an estimate, and it is anticipated that mine expansion produces considerably more IT than does exploration.

\section{SASKATCHEWAN}

As of September, 2009, there were 4,969 active mineral dispositions totaling 7,079,479 ha. In addition, there were 182 active potash dispositions covering 4,313,171 ha and 6,444 coal dispositions on 4,060,390 ha, the latter having increased markedly since 2008 after a major find was made in that year in the Hudson Bay area (Saskatchewan Ministry of Energy and Resources 2009). This would seem to indicate that there is considerable potential for incidental take in the mining sector, and the large amounts of exploration and mine development expenditures would seem to further support this expectation. However our analysis suggests that incidental take within the metals and mineral portion of the Saskatchewan mining sector is minor.

There are approximately 15 operating or soon-to-be operating mines in the province. They are roughly evenly-split between open pit and underground mines. A review of active mines in the province indicates that all of the nine major potash mines are underground (two are solution mines which inject water into the underground ore; the water becomes saturated and the mineral is extracted from the water). Many of the existing potash mines are expanding significantly in light of higher prices in 2007 and especially in 2008. Three of five operating and soon-to-beoperating uranium mines are underground, and there is one underground gold mine (Seabee). There are presently three operating coal mines in Saskatchewan; all of which are surface mines.

Exploration is especially active for gold, and there is a large open pit diamond mine under development in the central part of the province that will likely reach commercial production in a few years.

Discussions with government staff indicated that the average underground metal mine has an above ground footprint of roughly 50 ha, whereas an open pit mine would typically be between 250 and 350 ha in extent. This is consistent with the estimated average mine footprint of $0.5-2 \mathrm{sq}$ $\mathrm{km}$ provided in ArborVitae Environmental Services (2004). The potash mines are extensive and we have estimated that they might have a large footprint in the order of $200 \mathrm{ha}$. Therefore, the footprint of the existing mines, excluding access roads, is estimated at $3750 \mathrm{ha}$, as shown in Table 4. These mines have, in some cases, been operating for twenty years or more, and so there has been on average roughly one new mine per year. The average footprint is $233 \mathrm{ha} / \mathrm{mine}$.

\begin{tabular}{|l|c|r|r|}
\hline Mineral/mine type & \# Mines & $\begin{array}{l}\text { Footprint } \\
\text { (ha/mine) }\end{array}$ & Total Area (ha) \\
\hline coal & 3 & 350 & 1050 \\
\hline uranium (open pit) & 2 & 350 & 700 \\
\hline $\begin{array}{l}\text { uranium } \\
\text { (underground) }\end{array}$ & 3 & 50 & 150 \\
\hline
\end{tabular}




\begin{tabular}{|l|c|r|r|}
\hline potash & 9 & 200 & 1800 \\
\hline gold & 1 & 50 & 50 \\
\hline SUM & 18 & & 3750 \\
\hline
\end{tabular}

Table 4. Es timated footprint of operating mines in Saskatchewan (Incl Cigar Lake).

The run up in the price of potash that culminated in 2008 has led almost all of the potash mines in the province to expand significantly, most are in the process of doubling in size (Stilling, pers comm.), however most of the clearing associated with the expansions appears to have taken place. The consultant reviewed the Environmental Impact Statement assessments that had been prepared and approved in support of two significant potash mine expansions in Saskatchewan (K2 Tailings Management Area Expansion submitted by Mosaic Potash in January 2009; Potash Corp for its Rocanville West Expansion submitted August 2008) and a coal mine expansion project submission (Poplar River North Mine Extension Project submitted by Prairie Mines and Royalty Ltd in December 2008). These reports, provided by the provincial Environmental Assessment Branch, had varying levels of discussion relevant to incidental take.

The Rocanville West application requested permission to expand the operation by approximately 368 ha for a larger tailings management area to accommodate a proposed major increase in mining and mill capacity. The project assessment stated the following regarding the timing of disturbance: "Given the existing disturbance within the Project area from agricultural and industrial activities (e.g., brush clearing, modified pasture, and the PCS mine site including rail lines, roads, and other support infrastructures), wildlife is expected to have become habituated, or at least tolerate increased noise and activity levels. Construction for the new Service Shaft is scheduled to start before the breeding periods for sensitive species, such that they will naturally find alternate breeding areas."

The K2 expansion covers only 60 ha, and there were no wildlife issues noted; in fact, the EIS had very little to say about biology.

One of the major exploration projects in the province is being undertaken by Shore Gold, which is in the later stages of assessing a diamond resource in the central part of the province, east of Prince Albert. The mines are expected to be open pit, at least during the first number of years, and they should be quite large. The footprint of the Star - Orion South deposits (two pits) is expected to be 5000 ha, the Orion centre and north deposits are also approximately 5000 ha, and the Taurus deposit appears to be approximately 6500 ha. These mines would likely be developed over a multi-decade period.

Because the Shore Gold project has not yet developed its official resource estimate nor has it yet applied for an environmental assessment, it is estimated that approval for the mine is at least three years away. Therefore, going forward, we will use a baseline assumption that there will be one new mine developed each year, requiring an average area of 233 ha cleared for the mine and associated facilities each year.

Discussions with Saskatchewan government staff indicate that the amount of incidental take associated with coal mine development is also limited. In spite of the interest in the coal discovery, the south western part of the province lies within the Western Sedimentary basin, where coal is abundant and it frequently can be found close to or at surface. The 2008 discovery was some depth underground and the economics are not as appealing as developing a surface mine, hence the discovery is not expected to lead to the development of any new coal mines in the province. 
The three surface coal mines are in fairly close proximity and were last expanded in 1992, when a new electricity generating plant was built nearby. The mines tend to clear an area of about 1.6 $\mathrm{km}$ long by $100 \mathrm{~m}$ wide, extract the coal to a depth of some $30 \mathrm{~m}$, reclaim the area and then repeat the process adjacent to the previous operation. Concerns over climate change and associated regulations and potential carbon taxes, together with a lack of new demand sources for coal in Saskatchewan, have limited interest in the development of new coal mines. While the footprint of the existing mines does gradually shift, as described above, the amount of incidental take associated with coal mining in Saskatchewan is considered to be very low. This general assessment was supported by staff from the Saskatchewan Dept of the Environment, who stated that they encourage any land clearing to take place as early in the season as possible to enable birds to find another nest location (hopefully before they have built their first nest) (Riemer, pers. comm.). And it was mentioned that land clearance for mining would not be permitted during the nesting season if there was evidence that species of concern were nesting in the area.

The consultant reviewed the Environmental Impact Statement (EIS) reports that had been prepared and approved in support of a significant coal mine expansion - the Poplar River North Mine Extension Project submitted by Prairie Mines and Royalty Ltd in December 2008. The EIS stated that the existing Poplar River North area covered 4,340 ha, and the proposed extension was to include 3,038 ha, of which 1,711 ha will be disturbed by mine activity. Project life extends from 2010 to 2039 .

However, the EIS contained the following provision to minimize the potential for incidental take: "the removal of native vegetation will not be conducted between April 15 and August 15, where possible, to avoid the disruption of breeding grassland birds (SKCDC, 2007b); consultation with MOE will be conducted should salvage operations be required inside timing window restrictions ...". In addition, the EIS stated that it would "conduct drainage and alteration of wetlands in the fall to minimize the impacts on amphibian breeding habitat". Finally, vehicle speeds were limited to from $50-80 \mathrm{~km} / \mathrm{hr}$ to reduce the potential for wildlife-vehicle collisions. The set of proposals in the EIS hold out the prospect that there will be very little incidental take associated with this major expansion project.

The consultant was only able to make a rough estimate of the number of new mines or significant mine expansions planned for the province in the next five years - an average of one new mine, or the equivalent, is forecast. In other words, it will be assumed that 233 ha will be cleared annually for the mining of potash and metals. In addition, there is land clearing for coal production. The Poplar River North project will result in a significant amount of clearing - if all of the clearing is to take place within the first 15 years of the time period indicated, an average of 114 ha will be cleared annually. Because there are other strip mining operations in place, this area will be doubled to develop an annual estimate of 228 ha cleared for coal. It is assumed that all of this area will be grassland.

The information provided by various interviewees and suggested in the EIS' suggests that much of the land clearing that is done for mining takes place outside of nesting season. No quantitative estimates were obtained of what proportion of clearing activity took place in the nesting season the consultant interpreted the relevant information to suggest that between 5 and $20 \%$ of the land clearing takes place during the nesting season, and so estimates were made using these two proportions as upper and lower limits. As indicated above, a considerable amount of IT is thought to occur in grassland ecosystems, where the majority of the coal strip mining takes place. It was estimated that $80 \%$ of the cleared area is grassland, and $20 \%$ forest. The average clutch size figure was obtained from Garrison (1999). 
Table 5 shows the results of the analysis, with the high end estimate data shown in the table. The upper estimate of IT in the province due to mining is 745 birds, versus a low end estimate of 186 birds.

\begin{tabular}{|l|r|}
\hline Parameter & \multicolumn{1}{|c|}{ Sask } \\
\hline avg ann mine expan (ha) & 233 \\
\hline avg ann coal expan (ha) & 228 \\
\hline area cleared (ha/yr) & 461 \\
\hline \% forest cleared & 20 \\
\hline$\%$ grassland cleared & 80 \\
\hline$\%$ area cleared in nesting season & 20 \\
\hline forest bird density (pairs/ha) & 7.5 \\
\hline grassland density (pairs/ha) & 0.2 \\
\hline clutch size (eggs/nest) & 4.87 \\
\hline$\%$ destroyed during clearing & 100 \\
\hline IT estimate (upper) & 745 \\
\hline IT estimate (lower) & 186 \\
\hline
\end{tabular}

Table 5. Estimated Range of Incidental Take due to Mining in Saskatchewan.

This estimate is much lower than it was in British Columbia, due to two main factors - the majority of Saskatchewan mines are underground and so do not expand on a regular basis, and the majority of the cleared area was grassland, which has a lower density of birds than forest. Finally, B.C. has more mines than Saskatchewan.

\section{ONTARIO}

There is expected to be approximately 1 new mine per year for the next five years in Ontario. Data on footprint size was rather general - the Ontario Mining Association stated that the footprint for a mine was less than five square kilometers, but it was not clear how much less. The consultant used a range of footprint size of $1.0-2.5 \mathrm{sq} \mathrm{km}$.

The majority of new mine development is taking place in the north of Ontario, generally in forested lands. Using the average of Hobson et al (2009)'s nesting densities for Ontario yields an average of 7.8 nests/ha. The author could not find information on the timing of land clearing $-\mathrm{a}$ range of 10 to 30 percent cleared during nesting season was used.

Table 6 shows that the upper estimate of IT is almost 26,000 eggs and birds, versus a low end estimate of approximately 3,500 . The considerable width of the range is due to the relatively high degree of uncertainty around some key data points.

\begin{tabular}{|l|r|}
\hline Parameter & Ontario \\
\hline area cleared (ha/yr) & 2500 \\
\hline$\%$ forest cleared & 100 \\
\hline
\end{tabular}




\begin{tabular}{|l|r|}
\hline$\%$ grassland cleared & 0 \\
\hline$\%$ area cleared in nesting season & 30 \\
\hline forest bird density (pairs/ha) & 7.8 \\
\hline grassland density (pairs/ha) & 0.2 \\
\hline clutch size (eggs/nest) & 4.44 \\
\hline$\%$ destroyed during clearing & 100 \\
\hline IT estimate (upper) & 25974 \\
\hline IT estimate (lower) & 3463 \\
\hline
\end{tabular}

Table 6. Estimated Range of Incidental Take due to Mining in Ontario.

\section{NUNAVUt AND NORTHWEST TERRITORIES}

The Nunavut and Northwest Territories have a fairly long history of commercial mining, however a new wave was started in 1991 with Charles Fipke and Stuart Blussom's discovery of diamondbearing kimberlites on the shores of Lac de Gras in the Northwest Territories.

Currently, there are three diamond mines in the Northwest Territories - Ekati (owned by BHP Billiton), Diavik (owned by Harry Winston), and Snap Lake owned by de Beers. Nunavut had one diamond mine - the Jericho mine owned by Tahera - that closed in 2008 as it was losing money. The Meadowbank gold mine, which began operations in February 2010, is the only operating mine in Nunavut at present (April 2010).

There was considerable exploration in both territories during the commodity boom, but both are high-cost jurisdictions and the failure of the Jericho mine well illustrates the risks associated with mining in the two territories.

Nunavut is seen as being more friendly to mining than NWT. John F. Kearney, President of the NWT \& Nunavut Chamber of Mines (2010), stated that "Unfortunately, the NWT is getting a reputation as a difficult place to gain land access for exploration or to get permits for mining projects, or even for basic exploration programs, or to get permits within a reasonable time."

On April 10, 2010, the Globe and Mail ran a feature on mining in the Northwest Territories (The North scrapes bottom, Page B1). The report observed that exploration spending in the Northwest Territories had declined from \$148 million in 2008 to \$29 million in 2009. This was the steepest percentage decline in Canada. In sharp contrast, 2009 expenditures in Nunavut were \$189 million, and $\$ 75$ million in the Yukon, which is the first time in 15 years that the Yukon had seen more exploration expenditure than the NWT (NWT and Nunavut Chamber of Mines 2010).

The NWT and Nunavut Chamber of Mines (2010) continued by stating that: "For 2010, NRCAN's preliminary estimates show expected exploration expenditure in NWT at $\$ 66.3$ million, which will represent more than a $100 \%$ increase from 2009 . Of the [projected amount], it seems that $\$ 36$ million is projected for diamond mine appraisal, and presumably represents the feasibility study on Gahcho Kue, so actual projected pure exploration is again only about $\$ 30$ million. Expenditures in Nunavut for 2010 are estimated at \$238 million and the Yukon again at \$75 million."

There are two mines in an advanced stage of development in NWT - a rare earths mine being planned by Avalon Resources (Thor Lake) and a second diamond mine at Snap Lake (the Gahcho 
Kue mine), owned de Beers. Fortune Minerals is also working on developing a gold-cobaltbismuth project in NWT that is delayed at present due to a request by the Tlicho government for Fortune to wait until the Tlicho have completed land use planning for their traditional territory, which includes not only the mine but also a proposed road to the mine that is the main source of controversy. Given these legal issues, the timing of mine development, if it occurs, is difficult to forecast. That is all that is in the NWT pipeline for the next five years. Despite the greater receptivity to mining in Nunavut, there is probably no more than 2-3 new mines anticipated in that territory over the next five years.

There is a wide range of impact sizes associated with various mines. The existing diamond mines are very large. The Ekati mine had a footprint of 1758 ha in 2005 and that was planned to expand by 239 ha per year (Male and Nol 2005), so that it would be 2953 ha in 2010 if it kept to the planned rate of expansion. The Diavik mine has a $9 \mathrm{sq} \mathrm{km}$ footprint (www.diavik.ca). At the other end of the spectrum, the footprint of the Doris North gold project will be 41 ha of land (25.1 ha for road and building pad construction plus 15.4 ha as rock quarries) and an additional $13 \mathrm{ha}$ that will be flooded by the final water level in Tail Lake.

The Meadowbank gold mine (operated by Cumberland Resources), is a complex of three open-pit gold mines located about $70 \mathrm{~km}$ north of the community of Baker Lake. Access to the mine from Baker Lake is by a $115 \mathrm{~km}$ all-weather access road. The project submission to the Nunavut Impact Review Board (NIRB), which is the equivalent of the territorial environmental assessment review board, estimated that a total of 478 ha would be developed for the mine and access road, of which 288 ha was high suitability habitat for terrestrial birds. There was little discussion in the submission regarding timing of activities or impacts on migratory birds.

Estimates of potential incidental take were developed by reviewing the environmental assessment applications for new mines /mine expansions. These provided information relating to the mine footprint, seasonality of development and operational activities, the bird species that might be affected, and measures taken to mitigate impacts. While some of the EA reports provided more complete information than others, the total amount of evidence presents a consistent analysis of potential IT impacts.

Summary of EA Reports

Other projects that have recently gone to review by the NIRB include the Doris North project, the Baffinland Iron Mine Project and the Hackett River Project.

As indicated, the Doris North is a small project. Initially, it will be set up to redevelop an existing mine, requiring little additional land clearing. However, under these circumstances, the project would only operate for a total of 24 months; the intention is that some of the neighbouring deposits would prove to be viable and would be developed. The infrastructure that would be constructed at the mine and camp sites would consist of a mill, crushing plant, fuel storage tank farm, camp, office complex, workshops, power generation plant, and sewage treatment plant, as well as an all-weather airstrip. In addition, a $4.8 \mathrm{~km}$ road will be built from the mine to the sealift loading area and another $5 \mathrm{~km}$ road will be constructed to the appropriately named Tail Lake, where the tailings would be stored.

The Doris North Environmental Impact Statement (EIS) indicated that waterfowl and upland breeding birds were among the valued ecosystem components identified at an Elders Workshop. However, because most of the road and mine pad construction will take place during winter; incidental take will be the minimal. The EIS states that "Construction rock will be placed directly 
onto the tundra in winter to protect the permafrost when building roads, laydown areas and building pads" [italics added].

The following mitigation measures were included in the EIS:

- conduct land clearing in summer for site infrastructure (e.g., building pad construction and roads) outside of the breeding season (June 1 to August 1);

- prevent upland birds from nesting on mine infrastructure and man-made structures; and

- if a nest site is established and eggs are present, avoid the nest as much as possible and monitor for nest success.

The project being undertaken by Baffinland is the construction of a large, open pit iron ore mine located on north central Baffin Island. The ore would be shipped via rail to an all-season, deepwater port at Steensby Inlet. The rail line would be $143 \mathrm{~km}$, and be constructed by Baffinland, as would the mine facilities and Steensby port facilities. A tote road would also be available for transport /access. This project would necessitate the construction of significant infrastructure, but discussions of impact area and seasonality could not be found in the literature.

The Hackett River project is based on deposits of zinc, silver, copper lead and gold; the proposal is for two open pits and one underground mine. The mine would make use of the proposed Bathurst Inlet Port and Road, and a $23 \mathrm{~km}$ access road would connect the project site with the BIPR road. Total footprint or area disturbed was not reported in the EIS, but key sources of IT were discussed and mitigation factors provided (i.e., avoid sensitive areas, try to operate outside of sensitive time periods such as breeding season). In addition, the project operator will try to avoid stray light, limit vehicle speeds and road access to minimize bird-vehicle strikes.

In March 2010, the Canadian Zinc Corporation submitted a Project Proposal Report in support of the Environmental Assessment of the proposed Prairie Creek Mine, which is a proposed redevelopment of an old mine site in NWT. The report indicated that the current 52 ha footprint of the mine will be expanded by about 6 ha to accommodate a new waste rock pile, removing an area of black spruce-lichen habitat. The main mine access road, which is $175 \mathrm{~km}$ long, will have some re-design to improve safety and reduce environmental impacts - the work is expected to be undertaken in the first winter. The report did not speak to incidental take but did note for the SARA and COSWEIC bird species that the truck haul would be undertaken in winter, "well outside" of the breeding period.

\begin{tabular}{|l|l|l|}
\hline Territory & Mine & Footprint \\
\hline NWT & Ekati & 239 ha /yr expansion \\
\hline Nunavut & Doris North & 41 ha \\
\hline NWT & Canadian Zinc & $<20$ ha \\
\hline Nunavut & Hackett River & Increased by 6 ha \\
\hline Nunavut & Baffinland & unknown \\
\hline NWT & Nico & unknown \\
\hline
\end{tabular}

The forecast footprint and rate of creation of that footprint are unknown for the Baffinland and Nico projects, however they are likely to be relatively large since both are open pit mines and there are new access roads also proposed. It is unknown when either mine will enter construction. If each of the Baffinland and Nico mines was to have a $1 \mathrm{sq} \mathrm{km}$ footprint created within the next five years, and the rate of expansion at Ekati remains $239 \mathrm{ha} /$ year, then the expected total clearing in the two territories would be $3.25 \mathrm{sq} \mathrm{km}$, which is equivalent to an annual rate of clearing of $650 \mathrm{ha} / \mathrm{yr}$. This is the high estimate. On the other hand, if only one of 
the Baffinland or Nico mines goes ahead in the next five years, its foot print is 500 ha and the rate of expansion at Ekati slows to $100 \mathrm{ha} / \mathrm{yr}$, then the amount of clearing will be about $220 \mathrm{ha} /$ year. This is the low estimate.

\section{Bird Species and Densities}

The Meadowbank and Doris North EA submissions provided information about bird species and densities. In these studies, Lapland longspurs and horned larks were the most common birds observed. Other common migratory songbird species observed included redpolls, savannah sparrows, and America tree sparrows. The most common species of shorebirds were golden plovers, semipalmated plovers, and semipalmated sandpipers.

Assessment work undertaken for the Doris North project reported fifteen species of upland breeding birds during baseline surveys with densities ranging from 0.1 to $0.79 \mathrm{birds} / \mathrm{ha}$. Mean density estimates for individual species ranged from 0.01 birds/ha for semipalmated plovers to $0.18 \mathrm{birds} / \mathrm{ha}, 0.47 \mathrm{birds} / \mathrm{ha}$ and $0.79 \mathrm{birds} / \mathrm{ha}$ for American tree sparrows, savannah sparrows, and Lapland longspurs, respectively. Seven ptarmigan were observed in the 1997 aerial survey with a mean density of $0.35 \mathrm{ptarmigan} / \mathrm{km} 2$ (0.004 ptarmigan/ha), and was consistent with the low frequency of incidental observations throughout the year. Note that no horned lark densities are estimated, despite this being the most commonly observed bird species in the Doris North area.

The waterfowl assessment work reported that during all three years, Canada geese and whitefronted geese were the most abundant birds observed. Other common species included tundra swans, long-tailed ducks, Pacific loons and sandhill cranes. Thirteen species of ducks and geese, three species of loons, one swan and one crane species were observed during baseline surveys. Estimated waterfowl density ranged from 2.5 to $10.2 \mathrm{birds} / \mathrm{ha}$, presumably in appropriate habitat.

Of the 14 bird species considered to be breeding within the Meadowbank area, the Lapland longspur was by far the most common, averaging (i.e., of all 88 plots to date) approximately seven pairs per 16 ha plot or 43 pairs per 100 ha. Relatively few shorebirds were recorded during baseline surveys. The most common shorebird species was the semipalmated sandpiper, which was recorded in several extensive sedge meadows, often adjacent to small lakes and ponds, during the breeding bird surveys. Average number of pairs per plot was 0.2 or 1.5 pairs per 100 ha.

Densities of breeding bird species observed during the 2003 to 2005 breeding bird surveys indicate that the mine development will displace approximately 200 pairs $(0.43 \mathrm{pair} / \mathrm{ha})$ of Lapland longspurs, 60 pairs ( $0.12 \mathrm{pair} / \mathrm{ha})$ of horned larks, 20 pairs $(0.04 \mathrm{pair} / \mathrm{ha})$ of savannah sparrow, 15 to 20 pairs ( $0.035 \mathrm{pair} / \mathrm{ha})$ of rock ptarmigan, and minor numbers of other passerine species. The EIS focused heavily on the area of habitat loss, and devoted a relatively low amount of attention to direct mortality of birds through incidental take. It was noted that the ice-free period is only three months and so most of the construction activities are likely to be undertaken outside of breeding season.

\begin{tabular}{|l|l|l|}
\hline Species & Density Data (birds /ha) & $\begin{array}{l}\text { Average Reported Density } \\
\text { (birds/ha) }\end{array}$ \\
\hline Lapland longspur & $0.86(\mathrm{MB}), 0.79(\mathrm{DN})$ & 0.83 \\
\hline Horned lark & $0.24(\mathrm{MB})$ & 0.60 \\
\hline Savannah sparrow & $0.08(\mathrm{MB}), 0.47(\mathrm{DN})$ & 0.28 \\
\hline American tree sparrow & $0.18(\mathrm{DN})$ & 0.18 \\
\hline
\end{tabular}




\begin{tabular}{|l|l|l|}
\hline Semipalmated sandpiper & $0.06(\mathrm{MB})$ & 0.06 \\
\hline TOTAL & & 1.95 \\
\hline
\end{tabular}

If each species is assumed to lay four eggs once each year, then there are an average of 6 birds and eggs per ha during nesting season, plus some amount of waterfowl.

The high estimate is for an average area of 650 ha disturbed per year, implying that the maximum amount of potential incidental take will be 3900 birds and eggs, however it is estimated that $10 \%$ or less of the area will be cleared during breeding season, so that the maximum expected level of IT is 390 birds and eggs per year. At the low end, there is forecast to be 220 ha cleared per annum, indicating a potential IT level of 1,320 eggs and birds; $10 \%$ of this is 132 . As a result, the best guess of the level of IT per year over the next five years is between 132 - 390 eggs and birds per year.

\section{S UMMARY}

The analysis of incidental take due to metal and mineral mining activities is summarized below in Table 7, and expanded to other jurisdictions to develop a national estimate. The Ontario data were scaled by a factor of 2.28 to derive an estimate for Manitoba and all of eastern Canada (including Quebec) and the Saskatchewan data was scaled up by 1.19 to capture mining in Alberta. The basis for the scaling factors was mining expenditures for 2008 (Figure 2). The data from Nunavut and NWT were increased by $25 \%$ to account for the Yukon Territory.

\begin{tabular}{|l|c|c|}
\hline Jurisdiction & High IT Estimate & Low IT Estimate \\
\hline British Columbia & 32,453 & 6,722 \\
\hline Alberta & 887 & 221 \\
\hline Saskatchewan & 745 & 186 \\
\hline Ontario & 25,974 & 3,463 \\
\hline Manitoba, Quebec \& E Canada & 59,221 & 7,896 \\
\hline Territories & 488 & 165 \\
\hline CANADA & 119,768 & 18,653 \\
\hline
\end{tabular}

Table 7. Estimated Range of Incidental Take due to Mining in Canada.

There is considerable uncertainty in these estimates, primarily due to the lack of information about likely rates of mine footprint expansion, and the proportion of land cleared during the nesting season.

Another gap in the estimates is the lack of an estimate of incidental take due to exploration. It is anticipated that there is some IT associated with exploration, however much of the evidence obtained suggested that the largest proportion of vegetation and land clearing takes place outside of the breeding season. On the other hand, there is pressure on exploration departments to achieve results and it is likely that the dominant mindset is to undertake operations as soon as possible. Thus, if a company has determined that they would like to drill a specific target, and the first opportunity to do so is in spring, then it probably happens as soon as equipment is available and the site becomes accessible. Some of the later exploration work done to delineate a deposit would be captured in the amount of IT estimated for mines that reach production, however some initially promising deposits never become mines and any associated IT would not be captured in 
the estimates above. One final consideration is that it is expensive and time consuming to clear land and there have been a number of technological advances, such as heli-drills, that reduce the amount of disturbance, and the amount of associated IT. In sum, the range of IT levels shown in Table 7 is thought to be a reasonable range that probably bounds the true figure. 


\section{Incidental Take Associated with Aggregate Pits}

The term "pits and quarries" refers to the excavations required to extract sand, aggregate material, gravel, stone, cement, and other such products that are typically used in construction projects. Quarries are excavations into a rock bed where the rock is the desired product - limestone, granite and quartz are typical types of rock that are quarried. The sides of the quarry are frequently very steep and rocky, except for where the access road enters into the quarry. Pits are excavations into sand, gravel or other loose material that is the desired product - the slopes may be steep but are often graded. Other metals and minerals may be mined from pits but may also be extracted from below surface mines - these materials were dealt with in the Metals and Minerals mining analysis in the preceding section.

The majority of pits and quarries in Canada are on private land located close to major population centres, which are the main markets for these materials. Most aggregate materials are fairly widely abundant and the economics encourages extraction close to the location of use - i.e. near large cities. Statistics Canada (2008) reported figures for non-metallic mining and quarrying that included potash, asbestos, diamonds, and peat, which are all reviewed in other sections of this report. An estimate was developed of the value of stone, sand, gravel, clay, gypsum, and salt production from each province and territory from the Statistics Canada data. This was challenging because the production of some material types was not shown due to confidentiality concerns, and had to be estimated. The basic process was to subtract from the total production value the values associated potash, asbestos, diamonds, salt and peat. The results showed that approximately $55 \%$ of the value of stone, sand, gravel, clay, gypsum, and salt production originated in Ontario, with 22\% in Quebec, 9\% in British Columbia and 7\% in Nova Scotia (due largely to gypsum mining there). A considerable amount of production occurred in Alberta as well, and there were minor amounts of production in the other provinces, and negligible amounts in PEI, Saskatchewan and the three Territories. As a result, this section looks in detail at Ontario, Quebec, Alberta and British Columbia.

\section{ONTARIO}

The majority of the aggregate pits in Ontario are found on private land, especially when expressed in terms of their productive capacity. Aggregate pits on private land are required to be licensed. There are a significant number of aggregate pits on Crown land as well; tenure usually is provided through a permit. There are also what are known as Category 14 aggregate pits that are operated by forest management companies to provide aggregate for forest road construction and maintenance. Category 14 is actually an exemption from requirements that each pit be permitted - thus there are no permits for category 14 pits. However, the 2010 version of the Forest Management Planning Manual requests that all category 14 pits be identified, and thus the Forest Management Plans and Annual Work Schedules provide information on the number of pits that exist on each forest management unit. A review of these plans and reports found that many plan authors provided the information as a map product or in other formats that were not readily usable for the purposes of this project; a limited sample was obtained and used as the basis for estimation.

The table below provides a summary of key legislation and regulatory requirements. 


\begin{tabular}{|l|l|}
\hline Instrument & Location \\
\hline Governing Mining Legislation & Aggregate Resources Act \\
\hline Lead Provincial Dept & $\begin{array}{l}\text { Natural Resources, Aggregates and Petroleum } \\
\text { Resource Section. Category 14 pits are } \\
\text { transitioning to the Ministry of Forest Section } \\
\text { of the Ministry of Natural Resources. }\end{array}$ \\
\hline Crown Land Requirements & $\begin{array}{l}\text { A permit is issued under ARA. Forestry } \\
\text { exempt under category 14. }\end{array}$ \\
\hline Private Land Requirements & $\begin{array}{l}\text { Either owner applies or owner consents. A } \\
\text { licence is required under the ARA. A Class B } \\
\text { licence permits removal of less than 20,000 } \\
\text { tonnes/year, a Class A licence permits a greater } \\
\text { removal. }\end{array}$ \\
\hline Industry Association & $\begin{array}{l}\text { The Ontario Aggregate Resource Corp collects } \\
\text { the royalty and publishes an excellent annual } \\
\text { statistical report. } \\
\text { Ontario Stone, Sand and Gravel Assn }\end{array}$ \\
\hline Guidance re: Migratory Birds & $\begin{array}{l}\text { Section 5.00.17 in the June 1, 2007 Aggregate } \\
\text { Resources Program Manual requires the } \\
\text { consideration of migratory birds. }\end{array}$ \\
\hline
\end{tabular}

Ontario's aggregate sector is organized differently from that of other provinces in that The Ontario Aggregate Resources Corporation (TOARC) has been formed to collect royalties and other fees from the industry and distribute them back to the province. TOARC publishes an annual association report as well as an annual statistical report that handily exceed the available statistics from other provinces. Key for this study are data on the area under licence, under permit, and the amount of area under licence that is newly disturbed each year. Annual rehabilitation area is also provided.

The average area of land cleared on private lands between 2006 and 2008 was 992 ha/year. The area data were sub-divided to provide a total for each of the three Ontario regions (southern, central and northern). A disturbed area figure was not available for Crown land, however it was inferred by applying the annual rate of disturbance per area of private land under licence to the area of Crown land under permit. The resulting estimate of the area disturbed annually on Crown land was 1,380 ha/year, excluding Category 14 pits.

The majority of the provincial aggregate production takes place in southern Ontario, where the major demand centres are located. Interviews with staff of TOARC and MNR indicated that very little forest land was felled for aggregate pits in the south - the pit design would almost always avoid woodlots although some fencerows might be felled. Felling is largely done in winter. However, the overburden is primarily removed when it is unfrozen and dry, in part because the topsoil is stored and replaced during rehabilitation and removing it when wet or frozen damages its structure. As a result, the author estimated that most of the overburden removal takes place during nesting season; an estimate of $80 \%$ was used, which none of the interviewees contradicted.

In the central and northern regions, where more Crown land is cleared, it is anticipated that a minor proportion of the forest land is cleared during nesting season. Other than limits on operations caused by poor road conditions during spring breakup and the late fall, due to rain and freeze-up, there is no reason to think that there would be other sources of seasonality. Subtracting two months for each of the spring and fall operational breaks, and assuming an equal rate of 
clearing in the other months leads to an assumption that $25 \%$ of the forest would be cleared during nesting season.

I have also estimated proportions of forest and non-forest land cleared by general region - in the south, $95 \%$ of the land cleared is scrub, field, pasture or plowed agricultural land, and the remaining 5\% is assumed to be forest. In the central part of the province - Bancroft, Pembroke, Parry Sound, North Bay - the ratio was estimated at one-third grassland and brushland and $67 \%$ forest, while in the north it is estimated at 5\% grassland and brushland and $95 \%$ forest.

\section{Category 14 Pits}

So far, the analysis has not considered category 14 pits, so-called because they are authorized under category 14 of the Aggregate Resources of Ontario Provincial Standards. These pits are in fact exempted from permitting requirements so long as they meet a number of conditions, including having a size less than 3 ha. MNR staff suggested that the average site of a Category 14 pit might be 1.5 ha (Polhill, pers comm.). The Forest Management Planning process is beginning to report information about aggregate pits, however it is provided in different formats and different degrees of detail. Table 8 shows the results of a scan of 12 Forest Management Plans and/or Annual Work Schedules; data are not shown for the five plans in which no data could be found.

\begin{tabular}{|c|c|c|c|c|}
\hline \multirow[b]{2}{*}{ Forest } & \multicolumn{3}{|c|}{ Number of Pits } & \multirow{2}{*}{$\begin{array}{c}\text { Forest } \\
\text { Area } \\
\text { (ha) }\end{array}$} \\
\hline & Cat 9 & Cat 14 & All Pits & \\
\hline Dog River Matawin & 78 & 54 & 132 & 859079 \\
\hline English River & & & 61 & 1032771 \\
\hline Spruce River & & & 21 & 711159 \\
\hline Lake Nipigon & & & 1 & 900070 \\
\hline Nipissing & 103 & 39 & 142 & 761985 \\
\hline Romeo Malette & 99 & 14 & 113 & 629000 \\
\hline Crossroute & & & 150 & 874000 \\
\hline SUM & 280 & 107 & 620 & 5768064 \\
\hline
\end{tabular}

Table 8. Summary of Pit Information provided in seven Forest Management Plans.

In total, there are 107 active Category 14 pits on three of the forests, which collectively cover 5.8 million hectares. On average, there is one Category 14 pit per 21,000 ha on these three forests. Applying this same ratio on the entire Area of the Undertaking, which is 38.5 million ha, leads to an estimate of 1830 Cat 14 pits. The consultants made the assumption that the Category 9 pits would be covered in the TOARC statistics, however it is not known conclusively how true this assumption is.

Category 14 pits cannot remain open for more than ten years, and if the road used to access them is to be decommissioned, the pits must be rehabilitated beforehand. Since there is little incentive to close a pit early, especially when one might need to access it in future, it is likely that most category 14 pits are kept open for a considerable length of time - perhaps 7 to 10 years. This would suggest that the average number of pits opened in a given year would be between 261 and 183. At an area of 1.5 ha each, this suggests that the average area cleared annually for category 14 pits is on the order of 392 to 275 ha (the midpoint area of 333 ha was used, of which 83 ha was assumed to be located in central Ontario and he remainder in the north). It was estimated that these pits were all created by clearing forested land. 
Therefore, in 2008, the estimated area of Crown land disturbed in Ontario was 1,713 ha, or 17.1 sq km.

\section{Derivation of Incidental Take due to Land Clearing}

The calculation of the incidental take associated with land clearing for aggregate pits in Ontario is shown in Table 9. The upper rows are the estimated areas of land disturbed by region and ownership, below that the proportions of cover type are shown and the proportion of area cleared during the nesting season. Nest densities in the two habitat types were based on estimates of incidental take in Canada due to forestry operations (Hobson 2009), and on IT losses due to mowing and other mechanical operations in agricultural landscapes (Tews et al. 2009).

\begin{tabular}{|l|r|r|r|r|}
\hline \multirow{2}{*}{ Parameter } & \multicolumn{3}{|c|}{ Region } & \\
\cline { 2 - 5 } & \multicolumn{1}{|c|}{ South } & Central & North & TOTAL \\
\hline Area Disturbed (ha/yr) & & & & \\
\hline - Crown land & 2 & 228 & 1483 & 1713 \\
\hline - private land & 744 & 148 & 100 & 992 \\
\hline Total & 746 & 376 & 1583 & 2705 \\
\hline Prop'n grassland & 0.95 & 0.33 & 0.05 & \\
\hline Prop'n forest & 0.05 & 0.67 & 0.95 & \\
\hline $\begin{array}{l}\text { Prop'n grassland cleared } \\
\text { during nesting }\end{array}$ & 0.8 & 0.8 & 0.8 & \\
\hline $\begin{array}{l}\text { Prop'n forest cleared } \\
\text { during nesting }\end{array}$ & 0.25 & 0.25 & 0.25 & \\
\hline Forest density (pairs/ha) & 7.8 & 7.8 & 7.8 & \\
\hline Grassl'd density (pairs/ha) & 0.2 & 0.2 & 0.2 & \\
\hline No eggs/nest & 4 & 4 & 4 & \\
\hline IT grassland & 454 & 79 & 51 & 584 \\
\hline IT forest & 291 & 1965 & 11730 & 13986 \\
\hline Total IT & 745 & 2044 & 11781 & 14570 \\
\hline
\end{tabular}

Table 9. Incidental take in Ontario due to land clearing for pits and quarries.

Table 9 shows that there is a total of 14,570 young birds and eggs killed due to land clearing for aggregate pits. This is a meaningful amount of IT, and the majority of it takes place on Crown land in central and northern Ontario, with relatively little taking place on private land.

\section{Derivation of Incidental Take due to Pit Operations}

In addition to land clearing, incidental take also occurs due to pit operations. The killdeer nests in open sandy or gravelly areas and is known to nest readily in gravel pits. Bank swallows nest by digging dens within the pit walls - they do not nest in quarries where the pit faces are rock. Operations in the pits during nesting season could cause incidental take of both species.

\section{Killdeer}

The key factors in estimating the IT of killdeer consisted of:

- the number of pits and quarries with killdeer nests in them, and the number of nests per pit; 
- the proportion of pits and quarries that would have active operations in them during nesting season;

- number of eggs and young per nest; and

- the amount of mortality per nest caused by active operations.

Unfortunately there was little of the above data available.

The number of pits and quarries in operations was estimated to equal the number of licenses and permits. In 2008, there were 3,762 licences and 3,199 permits issued in Ontario (note that Category 14 pits are not included in these numbers). Some of the licence and permit areas are very large and probably have numerous pits, and there are some very large pits, so there may well be more than one nesting killdeer on some permits or licences. On the other hand, some pits will have no nesting killdeer.

The analysis above resulted in a mid-point estimate that there are 222 category 14 pits opened each year and if they remain open for eight years, then at any given time there will 1776 category nine pits open. In total therefore, there was an estimated 8,737 pits open at any given time. If we assume that $50 \%$ of these pits have a single active killdeer nest in them, and we estimate 4 eggs per nest, zero destruction of the adults, but complete loss of the brood if the nest is damaged, and that the harvesters operate in $25 \%$ of the available number of pits, then the amount IT caused by pit and quarry operations would be 4,369 eggs and very young birds.

\section{Bank Swallow}

The data required to estimate IT of bank swallow due to pit operations is similar to that used to estimate the IT of killdeer. The key differences are that bank swallows do not nest in quarries, since they cannot make their nesting cavities in rock, and that the swallows live in colonies that can be very large.

As in the case of the killdeer, there is relatively little information that can be gleaned from other studies. Most of the relevant information in the literature is associated with colony size, and those figures vary by region, and also have the risk of being out of date as Ontario bank swallow populations have declined significantly in recent decades; the Atlas of the Breeding Birds of Ontario, 2001-2005 reports that the Breeding Bird Survey has found an average annual rate of decline of $6.6 \%$ since 1981 .

The literature describes a considerable range in colony sizes, with figures ranging from less than 10 nesting pairs to several thousand. Larger colonies tend to be found along larger river systems. Garrison (1999) provides the following data regarding average colony size:

\begin{tabular}{|l|c|l|}
\hline Location & $\begin{array}{l}\text { Average colony size } \\
\text { (pairs of birds) }\end{array}$ & Citation \\
\hline California & $141-227$ & Lay-mon et al. 1988 \\
\hline Saskatchewan & 8 & Hiertaas 1984 \\
\hline Ontario & 45 & Peck and James 1987 \\
\hline Michigan & 58.6 & $\begin{array}{l}\text { Hoogland and Sherman } \\
1976\end{array}$ \\
\hline Alaska & $64.5^{*}$ & Hickman 1979 \\
\hline $\begin{array}{l}\text { Pennsylvania and } \\
\text { Vermont }\end{array}$ & $95.4^{*}$ & Spencer 1962 \\
\hline
\end{tabular}




\begin{tabular}{|l|l|l|}
\hline California & $367.8^{*}$ & Campbell et al 1997 \\
\hline
\end{tabular}

Table 10. Bank swallow colony size es timates from literature.

* number of burrows. Note that Garrison (1999) cited study results indicating that from $43-77 \%$ of burrows have nests in them.

Table 10 suggests that colony sizes are quite large in California (with two citations indicating similar results), and figures from that state probably do not apply to Ontario, although Garrison (1999) did report that one colony in Ontario had 1500 pairs and Cadman (pers. comm.. 2010) reported that one big pit he visited in southern Ontario had 2000 swallows in it in numerous nesting locations. The figure from Ontario is very close to a natural average figure of 42 pairs per colony (Erskine 1979), and so an average colony size of 44 pairs of birds will be used.

The proportion of pits with colonies in it varies geographically, with higher occupancy rates in southern Ontario locations. Cadman (pers. comm.. 2010) reported that in southern Ontario (he is working in Wellington County), virtually every sand and gravel pit has bank swallows in it. In 13 pits there was a total of 27 colonies, so there is usually more than one colony per pit.

The available data did not always differentiate pits and quarries. Production statistics from TOARC (2008) indicated that between 2001 and 2008, an average of 87.5\% of aggregate production in Ontario was from private land permits. In 2008, of 204 permits for above ground pits and quarries in southern Ontario, $169(83 \%)$ were for pits, $28(14 \%)$ for quarries and $7(3 \%)$ for combinations of the two. Therefore, the assumption will be made that in southern Ontario, bank swallows will be nesting in 169 of the permitted areas while none will occur in the quarries or combination pit \& quarry sites. Most of the Crown land licences are issued in central and northern Ontario, outside of the main range of bank swallow, and will not be considered in this estimate of bank swallow IT.

Based on the information provided by Cadman (2010), it will be assumed that there are two colonies per pit and that $20 \%$ of these colonies will be destroyed in a given year.

Therefore no. bank swallow colonies in southern Ontario aggregate pits $=169 * 2=338$.

No. bank swallow colonies destroyed $=0.2 * 338=68$

No. of bank swallow nests destroyed $=\#$ colonies $x \#$ nests/colony $=68 * 44=2992$

No. bank swallow eggs or young birds destroyed $=2992 * 4.44=13,284$

This calculation is based on an average clutch size of 4.44 in Ontario (Garrison 1999), and assumes that none of the parent birds are killed.

\section{Ontario Incidental Take Summary}

In summary, it is estimated that a total of 35,000 birds and eggs are destroyed by pit and quarry operations each year in Ontario. The majority of the losses are estimated to occur in southern Ontario, where there are many pits but also large populations of killdeer and bank swallows. The estimates show that roughly $60 \%$ of the IT takes place during operations, affecting killdeer and bank swallow, and roughly $40 \%$ occurring due to forest harvesting and overburden removal practices on land as it is being cleared.

\begin{tabular}{|c|c|c|c|c|}
\hline \multirow{2}{*}{ Parameter } & \multicolumn{3}{|c|}{ Region } & \\
\cline { 2 - 5 } & South & Central & North & TOTAL \\
\hline Area Disturbed (ha/yr) & & & & \\
\hline
\end{tabular}




\begin{tabular}{|l|r|r|r|r|}
\hline - Crown land & 2 & 228 & 1,483 & 1713 \\
\hline - private land & 744 & 148 & 100 & 992 \\
\hline Total & 746 & 376 & 1,583 & 2,705 \\
\hline Prop'n grassland & 0.95 & 0.33 & 0.05 & \\
\hline Prop'n forest & 0.05 & 0.67 & 0.95 & \\
\hline $\begin{array}{l}\text { Prop'n grassland cleared } \\
\text { during nesting }\end{array}$ & 0.8 & 0.8 & 0.8 & \\
\hline $\begin{array}{l}\text { Prop'n forest cleared } \\
\text { during nesting }\end{array}$ & 0.25 & 0.25 & 0.25 & \\
\hline Forest density (pairs/ha) & 7.8 & 7.8 & 7.8 & \\
\hline Grassl'd density (pairs/ha) & 0.2 & 0.2 & 0.2 & \\
\hline No eggs/nest & 4 & 4 & 4 & \\
\hline IT grassland & 454 & 79 & 51 & 584 \\
\hline IT forest & 291 & 1,965 & 11,730 & 13,986 \\
\hline $\begin{array}{l}\text { IT killdeer during } \\
\text { operations }\end{array}$ & 4,369 & 0 & & 4,369 \\
\hline $\begin{array}{l}\text { IT bank swallow during } \\
\text { operations }\end{array}$ & 13,284 & 0 & 0 & 13,284 \\
\hline Total IT & 18,398 & 2,044 & 11,781 & 32,223 \\
\hline
\end{tabular}

Table 11. Estimated Incidental Take due to Ontario Aggregate Pit Operations.

There are a few key areas of uncertainty, including such factors as the timing of when the land is cleared. There are also opportunities for further refinement of key factors such as elements of the calculation of in-pit mortality of killdeer and bank swallows.

\section{QUEBEC}

Aggregate Mining in Quebec is largely regulated by the provincial Environment Department, under the legislation identified below.

\begin{tabular}{|c|c|}
\hline Instrument & Location \\
\hline Governing Mining Legislation & $\begin{array}{l}\text { Environmental Quality Act (EQA) - } \\
\text { Regulation respecting Pits and Quarries }\end{array}$ \\
\hline Lead Provincial Dept & $\begin{array}{l}\text { Ministry of Sustainable Development, } \\
\text { Environment and Parks }\end{array}$ \\
\hline Crown Land Requirements & $\begin{array}{l}\text { Certificate issued by Minister of SDEP is } \\
\text { required under section } 22 \text { of EQA }\end{array}$ \\
\hline \multicolumn{2}{|l|}{ Private Land Requirements } \\
\hline \multicolumn{2}{|l|}{ Industry Association } \\
\hline Guidance re: Mig Birds & $\begin{array}{l}\text { The regulation }{ }^{1} \text { does not permit new pits to be } \\
\text { established within } 75 \text { m of water unless an } \\
\text { environmental impact assessment is done that } \\
\text { includes consideration of migratory birds. }\end{array}$ \\
\hline
\end{tabular}

\footnotetext{
${ }^{1} \mathrm{http}: / /$ www2.publicationsduquebec.gouv.qc.ca/dynamicSearch/telecharge.php?type=3\&file=/Q_2/Q2R2_A.HTM
} 
The consultant was unable to locate information pertaining to the area of aggregate pit activity in Quebec, including the number of leases or licences and the area affected. Instead, the incidental take estimates have been derived by pro-rating Ontario incidental take numbers by the ratio of pit operation activity in Ontario and Quebec. Statistics Canada (26-226-2006) reported that the value of aggregate extraction in Quebec was $45.7 \%$ of the comparable value in Ontario - 0.457 was the factor used to scale Ontario data and apply it to Quebec.

Hobson et al's (2009) figures on forest pair density, averaged between conifer and deciduous forest types, came to 8.45 pairs/ha, somewhat higher than in Ontario.

\begin{tabular}{|l|r|r|r|r|}
\hline \multirow{2}{*}{\multicolumn{1}{|c|}{ Parameter }} & \multicolumn{3}{|c|}{ Region } & \\
\cline { 2 - 5 } & \multicolumn{1}{|c|}{ South } & Central & North & TOTAL \\
\hline Area Disturbed (ha/yr) & & & & \\
\hline - Crown land & 1 & 104 & 678 & 783 \\
\hline - private land & 340 & 68 & 46 & 453 \\
\hline Total & 0.95 & 0.33 & 0.05 & \\
\hline Prop'n grassland & 0.05 & 0.67 & 0.95 & \\
\hline Prop'n forest & 0.8 & 0.8 & 0.8 & \\
\hline $\begin{array}{l}\text { Prop'n grassland cleared } \\
\text { during nesting }\end{array}$ & 0.25 & 0.25 & 0.25 & \\
\hline $\begin{array}{l}\text { Prop'n forest cleared } \\
\text { during nesting }\end{array}$ & 8.45 & 8.45 & 8.45 & \\
\hline Forest density (pairs/ha) & 0.2 & 0.2 & 0.2 & \\
\hline Grassl'd density (pairs/ha) & 4 & 4 & 4 & \\
\hline No eggs/nest & 207 & 36 & 23 & 267 \\
\hline IT grassland & 144 & 973 & 5,807 & 6,924 \\
\hline IT forest & 1,997 & 0 & 0 & 1,997 \\
\hline $\begin{array}{l}\text { IT killdeer during } \\
\text { operations }\end{array}$ & 6,071 & 0 & 0 & 6,071 \\
\hline $\begin{array}{l}\text { IT bank swallow during } \\
\text { operations }\end{array}$ & 8,419 & 1,009 & 5,830 & 15,258 \\
\hline Total IT & & & & \\
\hline
\end{tabular}

Table 12. Estimated Incidental Take due to Quebec Aggregate Pit Operations.

The resulting analysis shows that the incidental take associated with aggregate pit operations in Quebec is estimated at 16,086 eggs and young birds per year, of which about 6,100 are bank swallows and 2,000 are killdeer.

\section{ALBERTA}

While there is a reasonable amount of regulation of the aggregate pit sector in Alberta, there is considerably less monitoring and oversight on private land pits, which make up the majority of provincial pits (an estimated $66 \%$ of production comes from private land).

\begin{tabular}{|l|l|}
\hline Instrument & Location \\
\hline Governing Mining Legislation & $\begin{array}{l}\text { Public Lands Act for pits on Crown land; } \\
\text { Environmental Enhancement and Protection }\end{array}$ \\
\hline
\end{tabular}




\begin{tabular}{|c|c|}
\hline & Act (private land) \\
\hline Lead Provincial Dept & Sustainable Resource Development (SRD) \\
\hline Crown Land Requirements & $\begin{array}{l}\text { Surface Material Licences (SMC's) are for area } \\
\text { up to } 2 \text { ha and will be operated less than } 1 \text { year. } \\
\text { A Surface Materials Lease (SML) is issued } \\
\text { upon request, subject to approvals, when the } \\
\text { facility will be operating for } 10 \text { years or less, } \\
\text { and the lease area is less than } 80 \text { acres. When } \\
\text { the lease area }>80 \text { acres, tenders are invited for } \\
\text { the lease. }\end{array}$ \\
\hline Private Land Requirements & $\begin{array}{l}\text { Class I pits } \\
\text { - } \quad \text { five hectares or more in area } \\
\text { subject to the Code of Practice for Pits or } \\
\text { an existing approval under the } \\
\text { Environmental Protection and } \\
\text { Enhancement Act } \\
\text { - Pits with an existing approval must convert } \\
\text { to a registration by November } 1,2008 \\
\text { - Approximately } 550 \text { Class I pits on private } \\
\text { land in Alberta } \\
\text { Class II pits } \\
\text { - less than five hectares (on private land) } \\
\text { - } \quad \text { suby size (on public land) } \\
\text { and the Conservation and Reclamation } \\
\text { Regulation } \\
\text { Estimated } 1,500 \text { to } 2,000 \text { smaller pits } \\
\text { (private land) and } 650 \text { pits on public } \\
\text { Operators must comply with all } \\
\text { requirements of the Environmental } \\
\text { Protection and Enhancement Act and } \\
\text { its regulations and Codes of Practice. } \\
\text { In addition, they must comply with the } \\
\text { Alberta Water Act and all other } \\
\text { applicable provincial and federal laws. }\end{array}$ \\
\hline Industry Association & $\begin{array}{l}\text { Alberta Sand and Gravel Association does not } \\
\text { have any statistics - tried to collect pit area } \\
\text { data from its members who refused to provide } \\
\text { it. }\end{array}$ \\
\hline Guidance re: Mig Birds & $\begin{array}{l}\text { The } 2008 \text { Guideline references the Migratory } \\
\text { Bird Act and advises against tree clearing } \\
\text { between April } 1 \text { - June } 30 \text {. The Hay_Zama } \\
\text { Lakes complex requires specific precautionary } \\
\text { measures (see EUB Interim Directive 96-1). }\end{array}$ \\
\hline
\end{tabular}

Source: Alberta Sustainable Resource Development. undated. Acquiring Surface Material

Dispositions on Public Land. Available at http://www.srd.alberta.ca/ManagingPrograms/Lands/ 
Alberta Energy provided the consultant with a list of the Surface Material Lease (SML) and Surface Material Licence (SMC) data for the province, which included the area under lease or licence and the year of issuance. The area and issuance date information was summarized. The listing contained the surface leases for the oil sands operations - these were very large leases that were readily identifiable and removed from the calculation. There were a number of smaller leases covering areas in the $1-15$ ha range that were also issued to oil sands companies, and it was assumed that these were for pits to obtain material for road and other purposes. These leases were retained in the summary. Similarly, leases to two known peat moss extractors were also removed from the calculation.

The 2009 SMC area was 77.7 ha, and since these are one-year licences, 100\% of the entire area was assumed to be disturbed (Brenda Huxley, SRD, pers comm. Mar 31).

Figure 3 shows the area associated with SMLs, which are ten-year leases for larger deposits. The figure shows the average area of each type of lease for each year of issuance. Higher than normal average lease sizes occurred in 1998 and 2003, which are years when a large aggregate company, Athabasca Minerals, obtained some very large lease areas. Because the leases have a ten-year term, the ten-year running total area is shown, as the area under lease each year. The trend lines show that not only is there a greater area under lease over time, but the average lease area size is also increasing. During the last five years (2005-09), an average of 10,100 ha was under lease.

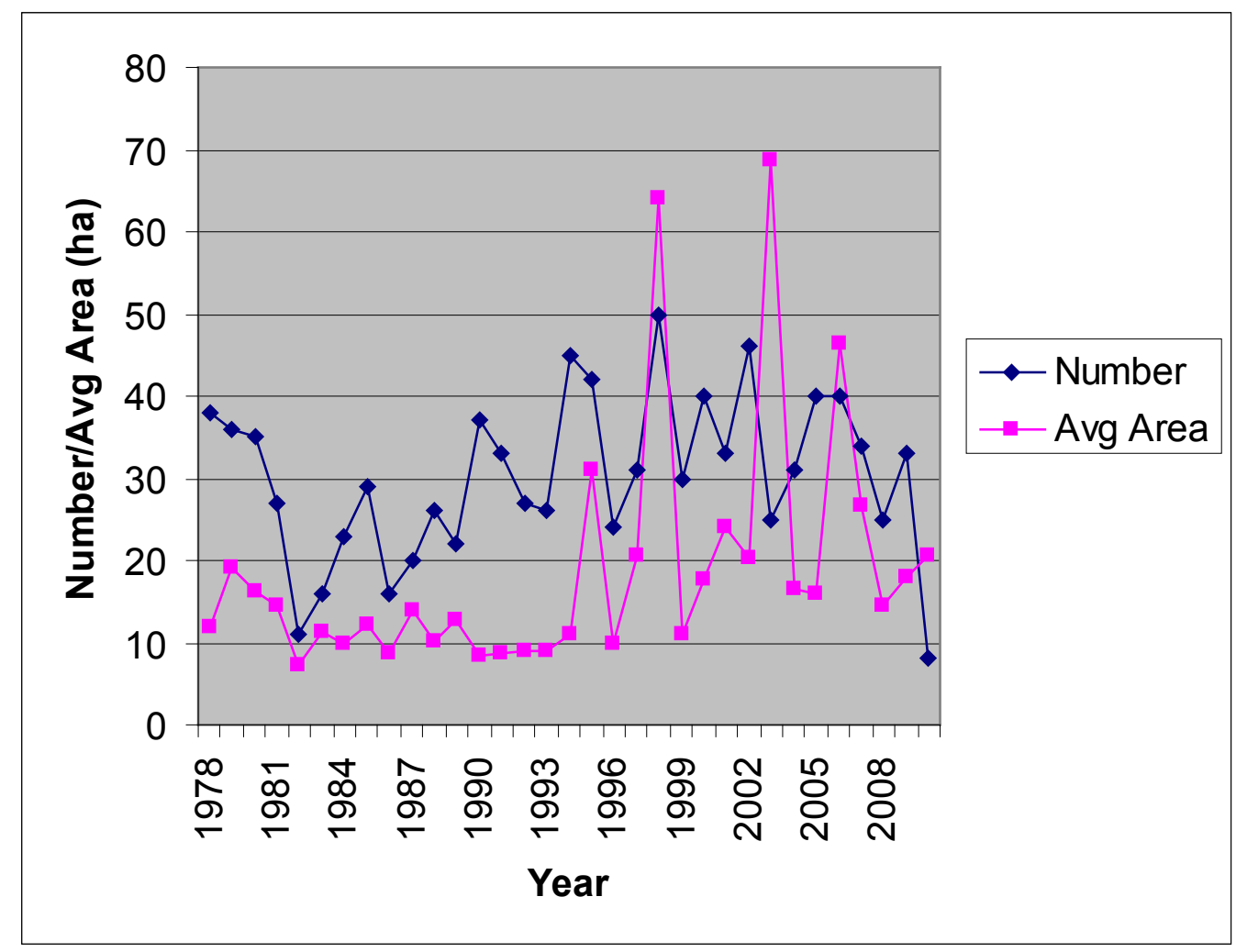

Figure 3. Number of New Leases/Near and Average Area per Lease (ha). (Source: Alberta Energy)

It was assumed that $8 \%$ of the surface area of each SML was disturbed annually - this was estimated to equate to an annual rate of extraction of ten percent reduced to provide allowance for 
inoperable areas, roads, buffers, etc. The calculation yields a result of 808 ha/year that is freshly disturbed, and with the area of licences added in, the total newly disturbed area per year is estimated at $886 \mathrm{ha} / \mathrm{yr}$.

In addition to the pits on public land, there is also a significant number of pits on private land. The Alberta government does not track or report on the area of pits in private land, nor are production figures available. However, a Crown land production estimate was available for the 2007-08 fiscal year, of 14,538,181.83 cubic yards. The weight of material in a cubic yard was set at 2600 pounds, based on crushed stone weighing $2500 \mathrm{lbs} /$ cubic yard, sand weighing 2500-2700 lbs/cubic yard, and gravel weighing $2700-2750 \mathrm{lbs} /$ cubic yard $^{2}$. Total provincial sand and gravel production in 2007 was preliminarily reported at 50,248,368 tonnes (Energy Resources Conservation Board and Alberta Geological Survey 2008). Therefore, Crown land production is only $34 \%$ of total provincial production, and the Crown land figures were tripled to scale them up to a provincial level.

In order to account for losses on private land pits, the Crown land IT estimated was tripled. We then increased the anticipated rate of IT per unit of private land disturbed, due to the relative lack of regulation on private land. With fewer regulations on private land, the manner in which pits are developed and exploited may be quite different than it is on Crown land. Our best guess estimate is that IT is $25 \%$ higher on private land, per unit of activity, than it is on Crown land (it is unlikely to be lower).

\begin{tabular}{|l|r|}
\hline \multicolumn{1}{|c|}{ Parameter } & TOTAL \\
\hline Area Disturbed (ha/yr) & \\
\hline - Crown land & 886 \\
\hline - private land & 1772 \\
\hline Total & 2658 \\
\hline Prop'n grassland & 0.95 \\
\hline Prop'n forest & 0.05 \\
\hline $\begin{array}{l}\text { Prop'n grassland cleared } \\
\text { during nesting }\end{array}$ & 0.8 \\
\hline $\begin{array}{l}\text { Prop'n forest cleared } \\
\text { during nesting }\end{array}$ & 0.25 \\
\hline Forest density (pairs/ha) & 4.6 \\
\hline Grassl'd density (pairs/ha) & 0.2 \\
\hline No eggs/nest & 4 \\
\hline IT grassland & 1616 \\
\hline IT forest & 611 \\
\hline $\begin{array}{l}\text { IT killdeer during } \\
\text { operations }\end{array}$ & 168 \\
\hline $\begin{array}{l}\text { IT bank swallow during } \\
\text { operations }\end{array}$ & 4838 \\
\hline Total IT & 9,247 \\
\hline
\end{tabular}

\footnotetext{
${ }^{2}$ The on-line sources for the weight/volume conversions were www.earthhaulers.com/faqs.html and www.abe.psu.edu/extension/factsheets/h/H2O.pdf .
} 
Table 13. Estimated Incidental Take due to Alberta Aggregate Pit Operations.

The Alberta Breeding Bird Atlas (1992) shows that bank swallows are found in central and southern Alberta, but are not common in the boreal part of the province. The Atlas reports that "Colonies are fairly common in cutbanks and gravel pits in central Alberta ..." and the populations may have increased locally because of human activity.

Only inferential information was found regarding the regional distribution of aggregate pits there are considerable concentrations of them near Edmonton and Calgary as well as near the oil sands. The area data above have all of the permits issued to oil sands companies removed but there may still be some boreal pits in the summary data - it is estimated that perhaps $20 \%$ of the area may be boreal. There is also a lack of local information regarding frequency of colonization of pits, colony size and disturbance rates. If the Ontario data are applied, the estimated IT of killdeer is 168 eggs and young birds, and 26,256 bank swallows. However, Table 10 shows that in Saskatchewan, the average bank swallow colony size is eight nests - using this figure gives a bank swallow IT estimate of 4,774. Our estimate is that the true figure lies somewhere in the mid point of this range.

\section{B RITISH COLUMBIA}

British Columbia has seen considerable construction over the past decade, associated with a booming property market and most recently the construction of the Olympic facilities and associated developments (e.g. Sea-Sky Highway widening). The aggregate sector is relatively closely regulated, with the main legislation and other requirements summarized below.

Authorization to develop the pit requires a pit management plan and site plan that requires current land use to be identified, final boundaries of excavation, etc. In theory this information is available to indicate the amount of various habitat types that will be removed, however it is generally not compiled.

\begin{tabular}{|l|l|}
\hline Instrument & Location \\
\hline Governing Mining Legislation & Mines Act \\
\hline Lead Provincial Dept & Energy, Mines and Petroleum Resources \\
\hline Crown Land Requirements & $\begin{array}{l}\text { Most quarry and pit operations receive a } \\
\text { Licence of Occupation to secure the land. This } \\
\text { lasts for five years; a replacement licence valid } \\
\text { for up to ten years may be granted. A permit } \\
\text { under the Mines Act is required to develop and } \\
\text { operate an aggregate pit - the application to } \\
\text { obtain the permit is a Notice of Work and } \\
\text { Reclamation Program. }\end{array}$ \\
\hline Private Land Requirements & Either owner applies or owner consents. \\
\hline Industry Association & $\begin{array}{l}\text { Aggregate Producers Association of British } \\
\text { Columbia provides a greater range of info but } \\
\text { no statistics relevant to migratory bird } \\
\text { incidental take. }\end{array}$ \\
\hline Guidance re: Migratory Birds & Not mentioned in 2010 guide \\
\hline
\end{tabular}

Source: B.C. Ministry of Energy, Mines and Petroleum Resources. 2010. Guide to Preparing Mine Permit Applications for Aggregate Pits and Quarries in British Columbia. February 2010. 
B.C. is divided into six regions for administrative purposes, although the number of regions is presently being reduced to five. The regions are: Northwest, Northcentral and Northeast, Kootenay, Southeast and Southwest. The web site http://www.empr.gov.bc.ca/Mining/MineralStatistics/Regional/southwest/Pages/Overview.aspx listed the number of aggregate pits in each region. A total of 470 pits were identified - in most regions, the website stated that at least the indicated number of pits were in operation. (No mention of active pits was made in any of the northern region overviews, hence this figure is assumed to apply to the southern and central part of the province, or in other words, the range of the bank swallow). The Mining and Minerals Division of the Ministry of Energy, Mines and Petroleum Resources kindly compiled data on total area disturbed by region by land use that resulted in land clearing for pits and quarries (e.g. for roads). Removing the area of pits from northeast and northwest region gave a total area of 3940 ha, of which $20 \%$ was assumed to be quarryland. It was assumed that on average, each pit is operated for five years and therefore, the annual area of newly cleared land equaled one-fifth of the total cleared land area, or 630 ha.

Hobson et al (2009) give the forest bird densities in BC as being 6.71 bird pairs/ha, which was used in this analysis.

\begin{tabular}{|l|r|}
\hline \multicolumn{1}{|c|}{ Parameter } & TOTAL \\
\hline Area Disturbed (ha/yr) & 630 \\
\hline Prop'n grassland & 0.65 \\
\hline Prop'n forest & 0.35 \\
\hline $\begin{array}{l}\text { Prop'n grassland cleared } \\
\text { during nesting }\end{array}$ & 0.8 \\
\hline $\begin{array}{l}\text { Prop'n forest cleared } \\
\text { during nesting }\end{array}$ & 0.25 \\
\hline Forest density (pairs/ha) & 6.71 \\
\hline Grassl'd density (pairs/ha) & 0.2 \\
\hline No eggs/nest & 262 \\
\hline IT grassland & 1480 \\
\hline IT forest & 188 \\
\hline $\begin{array}{l}\text { IT killdeer during } \\
\text { operations }\end{array}$ & 31674 \\
\hline $\begin{array}{l}\text { IT bank swallow during } \\
\text { operations }\end{array}$ & 33604 \\
\hline Total IT
\end{tabular}

Table 14. Estimated Incidental Take due to B.C. Aggregate Pit Operations.

Campbell (1997) reported that the bank swallow was uncommon to rare in the coastal region and on Vancouver Island, fairly common to locally very common in the southern and central interior and the Peace Lowland, and fairly common in the sub boreal interior and northern boreal mountain regions. Of 491 colony records, the range in number of nests is 3 to 3,035, with the most common number being between 15 and 75. Campbell (1997) reviewed Garrison's study of bank swallow in southern California (average of 269 pairs per colony) and opined that colonies 
tend to be larger in the mountains of western North America than in the remainder of the continent. A figure of 60 pairs per colony was used in this estimate. However, this somewhat larger colony size is counterbalanced by a lower average clutch size -3.51 for B.C. (Garrison 1999). Maintaining the assumption that there were 2 colonies per pit, and that $20 \%$ of the colonies were destroyed gives an estimate of IT of 31,675 eggs and young birds.

\section{NATIONAL RESULTS}

The amount of IT associated with aggregate pit operations in the other Canadian provinces and territories was calculated by extrapolating the total IT for the four provinces by the value of aggregate production in the remaining provinces (there is negligible aggregate production in PEI and the territories, hence the calculation covered the other provinces only).

The 2006 Statistics Canada publication 26-226 provided the value of sand and gravel production in most provinces and nationally (SIC 212323). The production from Ontario, Quebec, Alberta and British Columbia accounted for $90.3 \%$ of the national total. Thus, the IT associated with aggregate mining in the other provinces and territories was essentially $10 \%$ of the national total. The IT for the provinces not analyzed in detail was pro-rated based on relative value of sand and gravel production in Ontario and in eastern Canada and Manitoba, while the IT associated with Saskatchewan sand and gravel production was pro-rated from the Alberta rate using relative provincial value of production. The results of that calculation are shown in Table 14.

The results indicate that more than $50 \%$ of the incidental take associated with aggregate pits is due to the mortality of bank swallows, while killdeer mortality is approximately $7 \%$ of the total amount of IT. One of the main reasons why the IT due to clearing is relatively low is because the majority of trees are felled or pruned outside of nesting season, and because a substantial portion of the area cleared is grassland, which has a lower density of nesting birds than does forest.

As was described in the Ontario section, where the bank swallow determination was described in detail, there is a great deal of uncertainty regarding key variables such as proportion of aggregate pits with colonies, average colony size, proportion of pits with colonies that get operated during nesting season, and the proportion of the colony that succumbs to IT when operations take place during nesting season. Similar uncertainty is associated with the estimate of killdeer mortality.

\begin{tabular}{|l|r|r|r|r|}
\hline Province & $\begin{array}{c}\text { IT due } \\
\text { to } \\
\text { clearing }\end{array}$ & Killdeer & $\begin{array}{c}\text { Bank } \\
\text { Swallow }\end{array}$ & SUM \\
\hline Nfld, NS, NB & 2179 & 295 & 898 & 3373 \\
\hline Quebec & 7191 & 1997 & 6071 & 15259 \\
\hline Ontario & 32223 & 4369 & 13284 & 49876 \\
\hline Manitoba & 2177 & 295 & 897 & 3369 \\
\hline Saskatchewan & 308 & 23 & 661 & 993 \\
\hline Alberta & 2227 & 168 & 4774 & 7169 \\
\hline British Columbia & 1742 & 188 & 31674 & 33604 \\
\hline SUM & 48047 & 7336 & 58260 & 113643 \\
\hline
\end{tabular}

Table 15. Estimated Incidental Take of Bird Species of Interest in the $C$ anada due to Aggregate $\mathrm{P}$ it Operations using 1983 and es timated 2010 population figures. 


\section{Incidental Take Associated with Commercial Peat Harvesting}

Peat has been mined in Canada since the early 1890's, but it was not on a large scale until the Second World War led to the disruption of shipments from Europe, especially Scandinavia, who were traditional suppliers to Canada. ${ }^{3}$

New Brunswick is the largest peat producing province in Canada, producing 14 million bales in 2002-03, or 45\% of Canadian production (Canadian Sphagnum Peat Moss Association). There are approximately 140,000 ha of peatlands in New Brunswick; of this, approximately $70 \%$ is Crown land. The industry is concentrated in the NE part of the province. Peat mining on Crown Lands is covered by the Quarriable Substances Act.

In New Brunswick, a total of 5,448 ha were actively used for peat production, $77 \%$ of which were on Crown land (New Brunswick 2009). While the amount of area actively used is expected to gradually increase, it has been partially capped. In 2001, the New Brunswick government passed a regulation that would prevent the existing capacity of "basic" peat mining from increasing beyond the amount leased in 2001 (which included some areas that were not opened up). Since 2001, the province has only issued leases based on replacement - for additional area to be added, there must be value-added use, which is defined as producing a product with a value of at least $2 \mathrm{x}$ the value of basic peat.

Quebec's commercial peatlands tend to be found in the South Shore region; Quebec produced 10 million bales in 2002-03, equivalent to $32 \%$ of national production. Alberta is the third most significant producer province (4 million bales in 2002-03), followed by Saskatchewan and Manitoba (2 million bales each in 2002-03).

\section{PEAT HARVESTING}

To ready a peatland for harvesting, it must be drained which is accomplished by first removing the trees on site followed by ditching, often at 30 m intervals (Secretariat to the North American Wetlands Conservation Council Committee, 2001). Ditching is undertaken to facilitate the drying of the peat. After an interval of one or years when it is draining, the site is ready for production. The surface layer of vegetation may be removed prior to ditching, or a newer approach is the mix the surface vegetation with the upper layers of peat so that the mixture can be harvested and sold. This avoids the need to removed and dump the surface vegetation, saving expenses.

Peat is harvested using a large machine that disturbs the top layer of peat and then vacuums up the loosened dried peat. This approach was introduced in the 1960's as the cost of hand cutting and stacking became exorbitant. The average rate of mining is to remove $7 \mathrm{~cm}$ of depth per year (Thibault, pers comm.). The process is very weather dependent - a wet season curtails production opportunities.

The felling of the trees on a site where mining is to begin is done in winter, since that is the best time to move over the peatland, which at this point has not begun to drain. The other preparatory

\footnotetext{
${ }^{3}$ Source: www.gnb.ca/0078/minerals/Peat_Menu-e.aspx
} 
operations, such as ditching and stripping, are most often done in the late summer and fall - the ground has to be unfrozen and drier is better than wet. Because peat harvesting has stopped by the fall, the growers also find that by doing the preparatory operations in the fall they can keep their labour force employed longer. The majority of the labour is seasonal and extending employment allows the labourers to qualify for unemployment insurance. While most interviewees said that there is some possibility that stripping or other operations would be done in the fall, very little takes place then. Once the peat bog is in production, it does not provide nesting habitat.

As a result, this study concludes that there is negligible incidental take associated with peat harvesting in Canada.

When mining has been completed in an area, there is typically a layer of peat about 3 feet deep left. Sphagnum fragments are spread on the surface and these contain seeds of all types of bog plants - within five years, there is usually a good re-growth of typical species, ranging from herbaceous to lab tea, bog rosemary, and spruce. 


\section{Literature Cited}

Abraham, D., D. Pickard and C. Wedeles. 2010. Avian Incidental Take due to Roadside Maintenance Operations in Canada. Report Prepared by ESSA Technologies Ltd. and ArborVitae Environmental Services Ltd. for Environment Canada, Western Arctic Unit, Yellowknife. Error! Bookmark not defined. pp.

Alberta Energy Resources Conservation Board and Alberta Geological Survey. 2008. 2007 Alberta Mineral Exploration Highlights and Industrial Minerals Production Update.

Alberta SRD. 2008 edition. Guidelines for Acquiring Surface Material Dispositions on Public Land.

ArborVitae Environmental Services. 2004. Estimation of Future Carbon Losses from Deforestation in Canada. Prepared for NRCan as project 04-0927.

Blancher, P.J. 2002. Importance of breeding birds exported from Canada - Information on landbirds and shorebirds. Environment Canada. $46 \mathrm{pp}+$ appendices.

Campbell, Robert W. 1997. The Birds of British Columbia: passerines: flycatchers through vireos. UBC Press.

Canadian Intergovernmental Working Group on the Mining Industry. 2010. Overview of Trends in Canadian Mineral Exploration. Document prepared by Minerals, Metals and Materials Knowledge Branch, Mineral and Metals Sector, Natural Resources Canada.

Canadian Zinc Corporation. 2010. Prairie Creek Mine Developer's Assessment Report. Main Report Vol 1 of 4. Submitted to Mackenzie Valley Review Board in support of the Environmental Assessment of the proposed Prairie Creek Mine.

Hobson, Keith A. 2009. An Estimate of Incidental Take of Nests of Forest Birds in Canada due to Mining Operations. Draft dated 14 April 2009. Prepared for Environment Canada, Western Arctic Unit, Yellowknife.

Kennedy, J, P. Dilworth-Christie and Erskine. 1999. The Canadian Breeding Bird (Mapping) Census Database. Technical Report Series No.342, Canadian Wildlife Service, Ottawa, Ontario.

Garrison, Barrett A. 1999. Bank Swallow (Riparia riparia), The Birds of North America Online (A. Poole, Ed.). Ithaca: Cornell Lab of Ornithology; Retrieved from the Birds of North America Online: http://bna.birds.cornell.edu/bna/species/414

Miramar Hope Bay Ltd. Final Environmental Impact Statement: Doris North Project, Nunavut, Canada. Submitted October 28, 2005.

New Brunswick Department of Natural Resources. 2009. Peat Industry Review - 2008. Minerals and Petroleum Development Branch. March 2009. 6 pp.

NIRB Public Scoping Meetings for the Part 5 review of the Mary River Project 2009. 
NWT and Nunavut Chamber of Mines. 2010. Mineral Exploration Declining in Northwest Territories; Aboriginal Support for Regulatory Improvements, Government Investment in Infrastructure. News release April 15, 2010

Source: Ontario Ministry of Natural Resources. 2007. Aggregate Resources Program Manual. June 1, 2007. Available on-line at http://www.mnr.gov.on.ca/en/Business/Aggregates/2ColumnSubPage/266561.html

The Ontario Aggregate Resources Corp (TOARC). various years. Statistics.

The Ontario Aggregate Resources Corp (TOARC). various years. Annual Report.

Polhill, Brian. 2010. Forest Management Planning Section, Ontario Ministry of Natural Resources.

Sabina Silver Corporation. Hackett River Project, Nunavut: Project Proposal Report. Submitted January 2008.

Saskatchewan Ministry of Energy and Resources. 2009. Saskatchewan Exploration and Development Highlights 2009. (28 pp)

Saskatchewan Ministry of Energy and Resources. 2009. Saskatchewan Operating Mines List. Mines Branch, Mining and Minerals Statistics. February 2009. 15 pp.

Sauer, J. R., J. E. Hines, and J. Fallon. 2008. The North American Breeding Bird Survey, Results and Analysis 1966 - 2007. Version 5.15.2008. USGS Patuxent Wildlife Research Center, Laurel, $M D$

Secretariat to the North American Wetlands Conservation Council Committee. 2001. Canadian Peat Harvesting and the Environment ( $2^{\text {nd }}$ edition). Sustaining Wetlands Issue Paper 2001-1. Published in partnership with the Canadian Sphagnum Peat Moss Association and Environment Canada.

Semenchuk, Glen Peter. 1992. The Atlas of Breeding Birds of Alberta. Nature Alberta.

Statistics Canada. 2008. Non-metallic Mineral Mining and Quarrying. Cat. 26-226-X. October 2008.

Tews, J., Mineau, P. \& Bert, D. G. 2009 DRAFT Avian mortality related to mowing and other mechanical operations in agricultural landscapes, pp. 41: Environment Canada.

Tyhee Development Corp, 2010. Tyhee Development Corp provides Pre-feasibility Guidance and Exploration Update on the Clan Lake Zone, Yellowknife Gold Project, NWT, Canada. News release April 19, 2010.

People contacted:

Mr. Jacques Thibault, DNR New Brunswick at 506 547-2070 Jacques.Thibault@gnb.ca 
Dr. Line Rochefort, head of Peatland Ecology Research Group, Laval University

Jean-Pierre Savard, Canadian Wildlife Service, Environment Canada

Paul Short, President, Canadian Sphagnum Peat Moss Association. 


\title{
Avian mortality from pesticides used in agriculture in Canada
}

\author{
Pierre Mineau ${ }^{1}$ \\ ${ }^{1}$ Science and Technology Branch, Environment Canada, Ottawa, ON K1A OH3

\section{Executive Summary}

A serious impediment to estimating the impact of pesticides on migratory birds is the lack of comprehensive pesticide use data. Canada is one of the few developed countries that do not collect such information. A clear recommendation of this report (and of many others) is that Canada should establish a pesticide use reporting system.

Based on areas in various crop types and on low, average and high pesticide use patterns for those same crops in the US, our best estimate for the incidental take from pesticides in Canada is between 0.96 and 4.4 million bird annually. This estimate assumes a kill rate of approximately $0.52-2.4$ birds per hectare - the range from several industry studies carried out in typical farmland. A very approximate expert opinion is that a nest could be lost for every 4 birds killed. Given average nest success rates, this would add $50 \%$ more individuals to the above total.

The number of birds killed by pesticides has been decreasing as more toxic products are slowly being replaced for human health reasons. However, several potential impacts of pesticides, namely reproductive and indirect effects are not included in this total. Large kills of migrating birds are also not considered here although this would be more of a problem for a full accounting of pesticide impacts under US conditions than for Canada.

Approximately half of the total estimated kill is in Saskatchewan. The impact from pesticides is thought to be a clear contribution to the steep decline shown by several of grassland/farmland species. Because birds are killed on the breeding grounds, and because both adults and nests are vulnerable, this impact is proportionately higher than similar estimates derived for other sources of mortality.

Mitigation of kills is relatively easy. The products that have a high probability of causing avian mortality have been identified. In most cases, substitution products of lower toxicity to birds already exist. Regulatory inaction is the only impediment to a reduction of the direct incidental take. Chronic and indirect effects will be slightly harder to mitigate although here also, much information exists on which products carry the highest risk. 


\section{Introduction}

Pesticides have been documented to impact birds in many different ways (Mineau 2003). The continued use of acutely toxic products (primarily insecticides) has been shown to kill birds regularly and predictably even when used according to label directions (Mineau 2004). This can be a dramatic event when birds are killed in large numbers on migration. Most of the time, however, birds breeding at low density on the agricultural landscape are killed singly or in small groups and these losses go unnoticed.

Pesticides can also have indirect effects, notably the removal of valuable plant and/or insect components of agro-ecosystems necessary for successful reproduction. Finally, several products have the potential to affect bird sub-lethally, especially their long-term survival and reproductive fitness.

Modern pesticides have limited ability to bio-concentrate and bio-accumulate; most are readily metabolised in warm blooded organisms. There are some exceptions (e.g. rodenticides) but we lack enough information to assess the cumulative impacts of the latter ${ }^{1}$. The only 'legacy' aspects of pesticides are therefore the habitat modification (simplification) aspects that form part of the indirect effects.

It is only possible to quantify the direct lethal impact on birds and even this presents special challenges. One of the main difficulties is that Canada does not systematically collect pesticide use information. Partial surveys are available for some provinces but our knowledge of what pesticides are used where and in what quantity remains fragmentary and incomplete. It is possible to draw parallels to US agriculture where good pesticide use data are available but, since insect pressure tends to increase in warmer climates, extrapolation is difficult.

There have been very few attempts to estimate the total incidental take resulting from direct intoxications following the use of toxic pesticides anywhere. Pimentel (1992), in an oft-cited study, estimated that pesticide-induced direct mortality numbered approximately 67 million per year in the U.S. He based this estimate on the fact that 160 million ha of cropland received a very heavy dose of pesticides per year ( $3 \mathrm{~kg}$ a.i./ha on average - including a number of very toxic pesticides), a breeding density of 4.2 birds per ha (from census plot data) and a conservative kill estimate of $10 \%$ of exposed birds. This estimate ignores kills of wintering birds which could be substantial (Mineau and Whiteside 2006). Also, some of the largest kills recorded in North America have been of migrants (e.g. Lapland longspurs) which would not be captured in estimates based on breeding densities in farmland.

\footnotetext{
${ }^{1}$ Research on the impact of rodenticides has been going on for several years in S\&T. It should be noted however that the main species under threat are non-migratory raptorial species (e.g. buteos, eagles, various owl species).
} 
The insecticide carbofuran ( uradan $^{\mathrm{TM}}$ ) has been more studied than any other insecticide and can stand as the 'poster-pesticide' for bird mortality. Studies on a granular formulation of carbofuran as well as search efficiency and scavenging studies were used to provide an estimate of bird mortality per treated surface (Mineau 2005). Two major field studies, both from the U.S., were retained for purposes of extrapolation. Estimated kill rates were 3.05 birds per ha for an lowa site (once raw carcass counts were corrected for scavenging and for unsearched areas of the field) and 15.9 per ha for an Illinois site. A third study gave estimates that were simply too high to lead to a credible wide-ranging kill rate, a full 799 carcasses of a single species (Horned lark) having been recovered from slightly more than 100 ha of crop. It was estimated that, at the height of its popularity, in the late 70 s to mid 80 s, this single product was killing approximately 17 to 91 million songbirds annually in the 32 million ha of U.S. corn fields alone ${ }^{2}$.

Use of similar products in canola in the Canadian prairies was found to be correlated with regional declines in several grassland species (Mineau et al. 2005) even though the maximum proportion of treated cropland averaged about $3 \%$ and never exceeded $7 \%$ in any region of the prairies.

We will attempt to estimate the total number of birds killed by pesticides in Canada. Because most of the mortality is of breeding birds, there is an associated loss of nests and breeding potential which can be estimated also.

\section{Methods}

As mentioned above, this type of analysis is exceedingly difficult to do in Canada because we are one of the few developed countries around the world that does not assemble good pesticide use or sales statistics. In order to assess the likelihood of pesticide mortality for any given application of a pesticide, the following procedures were followed:

\section{Estimating the toxicity of pesticides to birds}

Very few species are typically tested for their sensitivity to pesticides. Interspecies differences in susceptibility can be very large. Also, the number of species tested with any given product can bias any toxicity estimate which is based on the most sensitive species tested. What is needed is a field-relevant unbiased measure of toxicity that can be used to provide a fair comparison of the kill potential of all registered pesticides. As a first step, a measure of acute pesticide toxicity for sensitive bird species ranging from 20 to 1,000 grams (a weight range that covers most bird species found dead in farm fields) was obtained by applying species sensitivity distribution techniques (Mineau et al. 2001). A value called the $\mathrm{HD}_{5}$ ('Hazardous Dose at the $5 \%$ tail of the species distribution') was derived. The $\mathrm{HD}_{5}$ is

\footnotetext{
${ }^{2}$ The product is no longer registered in Europe, the U.S. or Canada but continues to be used heavily in the developing world including countries of Latin America where many of our migrants winter.
} 
the amount of pesticide in $\mathrm{mg}$ of chemical per $\mathrm{kg}$ of body weight estimated to lead to $50 \%$ mortality in a species more sensitive than $95 \%$ of all bird species, calculated with a 50 percent probability of over- or underestimation. The $\mathrm{HD}_{5}$ was calculated mathematically where several toxicity values exist, or extrapolation factors were applied to single (or even multiple combinations of species-specific toxicity values see Table 1 in Mineau et al. 2001).

\section{Estimating the proportion of cropland at risk of sustaining a bird kill}

\section{First approach}

The probability of finding a bird kill (of any size) following a pesticide application was derived from models based on a large sample of empirical field studies where known insecticides were applied and searching was carried out to detect casualties. Models were developed for field and orchard crops separately. Because few of the studies were quantitative in nature, logistic modeling was used and the output of the models is the likelihood that a kill of undefined size would occur and be found assuming an adequate search effort ${ }^{3}$. Aside from the $\mathrm{HD}_{5}$ values, the models uses application rate, as well as physico-chemical constants such as octanol-water partition coefficient, molecular weight and size as well as the ratio of rat oral to dermal data, when available (details provided in Mineau 2002). Independent validation of the model for a sample of studies in field crops indicate that better than $81 \%$ of studies were correctly classified - as to whether they gave rise to mortality or not.

Using these models, Mineau and Whiteside (2006) analysed the insecticide use pattern for all U.S. crops on a State by State basis. They provided a minimum, weighted average and maximum proportion of each crop area where bird lethality was expected. It is possible to estimate the proportion of Canadian cropland at similar risk if we assume that pesticide use conditions in at least one of the censused U.S. States correspond to how the crop is treated in Canada.

Crop data for Canada was obtained from the 2006 quinquennial census of agriculture (Statistics Canada 2009).

Inadequate and incomplete recording of seed treatment chemicals as well as our inability to fit these uses into existing risk models means that they are essentially ignored in the estimates.

\section{Second approach}

Models developed in Mineau (2002) and described above were modified to take into account the addition of a few more field studies and a recent re-evaluation of all the component agricultural studies by a panel of four evaluators mandated by

\footnotetext{
${ }^{3}$ Carcass searches in most field studies employ lines of searchers systematically covering the field area as well as search dogs on occasion. The probability of seeing kills otherwise is negligible.
} 
the European Food Safety Authority (EFSA 2008). Mineau (2002) argued for the importance of dermal exposure when assessing the field data; however, because of the uncertainty surrounding dermal exposure to new classes of insecticides for which field studies do not exist (EFSA op. cit.), the basic risk model was modified to consider only the toxicity of the various pesticides to birds (here the $\mathrm{HD}_{5}$ ) in arriving at a probability of kill.

This simpler single model was applied to a summary of Canadian insecticide use assembled from disparate provincial sources of information on pesticide sales or use (Brimble et al. 2005). Pesticide sales or use data were not available for Québec (because several active ingredients are combined before the data are released) or for Saskatchewan. It should be noted that the quality of the data emanating from the other provinces is uneven and of unproven quality.

Because some of this survey information was already dated in 2005 , several listed insecticides are no longer registered today. These were removed from the list. Without any knowledge of their replacements, a full accounting of insecticide use in Canada is not possible and whatever kills are estimated must be under-estimates.

Application rates per hectare were obtained from pesticide labels. In reviewing available labels for each active ingredient, we tried to retain modal application rates - those rates that came up the most frequently, especially foliar rates of application associated with large area field crops. Mean application rates were calculated when modal rates were not evident (Appendix 2).

Most or all seed treatment pesticides currently registered and marketed in Canada were not included in the provincial totals. The risk from several of these compounds appears to be high in Canada (Smith 2006) although no field studies have been carried out and these uses therefore fall outside of the field models created to estimate the risk of mortality. Our inability to adequately assess the risk to birds from the growing use of seed treatments is a serious regulatory issue - as well as a serious gap for the purpose of this exercise.

\section{Estimating the number of birds involved in a kill}

As mentioned earlier, the majority of field studies provide a very poor basis on which to base a quantitative estimate of kills. Several field studies were carried out with carbofuran - either the liquid or granular formulations; in a few cases, alternative pesticides were tested as well under the same conditions (FMC 1989a,b summarised in Mineau 1993). Kills resulting from the use of the granular formulation were already quantified for the U.S. and reported above. Because the silica-based granular formulation of carbofuran is a rather unusual product (because of the combination of high toxicity and a granule base very attractive to birds) and is no longer registered in Canada, the studies reporting on spray applications will be used instead. 
One substantial advantage of using these studies to try to arrive at a quantified estimate of a 'typical' kill is that carbofuran, being a carbamate insecticide, kills more quickly than most other toxic insecticides. With several other products, birds are more likely to ingest a lethal dose but die later away from the field area, or ingest a dose which might have been sub-lethal had the bird not been subject to delayed mortality as a result of cold, food stress or predation (reviewed in Mineau 2003). Even with this rapid mode of action, some birds have been shown to leave the field area and die in surrounding fields and field margins. Also, these studies were carried out by the same group of experimenters under similar conditions, search plots were cleared of old carcasses prior to the study to minimise any confusion over the attribution of mortality to the pesticide and search and scavenging rates were well assessed in each plot by means of 3-day old black chicks of domestic chickens - a reasonable stand in for small to medium sized songbirds.

Table 1 below gives raw unadjusted rates of carcass counts for these carbofuran and associated ${ }^{4}$ field trials. In every case, the search area was divided equally between the field and surrounding non field habitat. Unlike the granular formulation, the liquid formulation of carbofuran is still registered in Canada. Chlorpyrifos and methomyl are also two major use insecticides in this country.

Table 1. Uncorrected kill rates for several pesticide field studies carried out under standardised conditions of searching employing teams of observers and search dogs.

\begin{tabular}{|l|l|l|l|}
\hline Pesticide & Crop & Location & $\begin{array}{l}\text { Uncorrected kill rate } \\
\text { (carcasses/searched } \\
\text { ha) }\end{array}$ \\
\hline carbofuran & corn & Nebraska & 0.43 \\
\hline carbofuran & corn & Texas & 0.53 \\
\hline carbofuran & alfalfa & Kansas & 0.47 \\
\hline carbofuran & alfalfa & Oklahoma & 0.22 \\
\hline chlorpyrifos & alfalfa & Kansas & 0.22 \\
\hline $\begin{array}{l}\text { Chlorpyrifos and } \\
\text { methomyl }\end{array}$ & alfalfa & Oklahoma & 0.53 \\
\hline
\end{tabular}

Table X. Uncorrected raw kill rates observed in several field studies reviewed in Mineau (1993).

Carbofuran is quite acutely toxic to birds so extrapolations based on this pesticide might be considered worst case. However, plots treated with chlorpyrifos, an insecticide of much lower acute toxicity to birds had similar levels of mortality. Also, because our methods of estimating the number of kills in Canada already factor in toxicity to estimate the probability that a kill will occur, it could be argued

\footnotetext{
${ }^{4}$ Alternate pesticides were applied to control plots in these studies.
} 
that the actual body count per hectare, when a kill does occur, is less a factor of toxicity and more dependant on the number of birds frequenting the field and potentially exposed.

Search rates and scavenging were measured in detail in these studies ${ }^{5}$. Plot by plot estimates vary widely but overall means are provided below for the combination of all habitats surveyed in the studies: fields, field margins and roads for the corn study; fields and field margins in the case of the alfalfa studies (Table 2).

Table 2. Measured rates of search efficiency and scavenging.

\begin{tabular}{|l|l|l|l|l|}
\hline Pesticide & Crop & $\begin{array}{l}\text { Search } \\
\text { efficiency } \\
\text { averaged } \\
\text { among all } \\
\text { habitat types }\end{array}$ & $\begin{array}{l}\text { Scavenging } \\
\text { rate averaged } \\
\text { among all } \\
\text { habitat types }\end{array}$ & $\begin{array}{l}\text { Combined } \\
\text { detection } \\
\text { rate }\end{array}$ \\
\hline corn & Nebraska & 0.22 & 0.19 & 0.18 \\
\hline corn & Texas & 0.37 & 0.26 & 0.27 \\
\hline alfalfa & Kansas & 0.50 & 0.16 & 0.42 \\
\hline alfalfa & Oklahoma & 0.46 & 0.21 & 0.36 \\
\hline
\end{tabular}

These are one time search and scavenging correction estimates. In theory, repeated visits to the site (daily searches for a week were carried out in the studies outlined above) increase the probability of finding carcasses not found during a previous search. However, because of the skewed nature of carcass life expectancies (fresh carcasses disappear quickly, older carcasses cease to be of interest to scavengers), attempts to calculate overall probabilities of detection by using mean carcass 'life-expectancies' have generally been unsatisfactory and heavily biased (Smallwood 2007). A carcass missed on the first day of searching and subject to a further 24 hours of scavenging has a rapidly decreasing probability of being detected on subsequent days. Also, if a carcass was not found on the first search day, it is likely well hidden and the probability that it will be found on subsequent days is considerably less than the average search rate would suggest. For these reasons, simple one time estimates were used here to estimate the number of birds that would have gone undetected in the field studies (Table 3).

\footnotetext{
${ }^{5}$ The help of Mélanie Whiteside is gratefully acknowledged for extracting the relevant information from lengthy industry reports.
} 
Table 3. Corrected kill rates for several pesticide field studies.

\begin{tabular}{|l|l|l|l|l|}
\hline Pesticide & Crop & Location & $\begin{array}{l}\text { Uncorrected kill } \\
\text { rate (carcasses } \\
\text { / searched ha) }\end{array}$ & $\begin{array}{l}\text { Corrected } \\
\text { kill rate } \\
\text { (carcasses } \\
\text { per ha) }\end{array}$ \\
\hline carbofuran & Corn & Nebraska & 0.43 & 2.4 \\
\hline carbofuran & Corn & Texas & 0.53 & 2.0 \\
\hline carbofuran & alfalfa & Kansas & 0.47 & 1.1 \\
\hline carbofuran & alfalfa & Oklahoma & 0.22 & 0.61 \\
\hline chlorpyrifos & alfalfa & Kansas & 0.22 & 0.52 \\
\hline $\begin{array}{l}\text { Chlorpyrifos } \\
\text { and methomyl }\end{array}$ & alfalfa & Oklahoma & 0.53 & 1.5 \\
\hline
\end{tabular}

I propose to use the range of mortality rates calculated from these studies to quantify mortality when the risk model predicts that mortality will occur. For example, if the logistic model predicts that a certain pesticide application has a 0.50 probability of mortality based on the combined sample of field studies, and assuming that the pesticide is applied to 100,000 ha, the low and high estimates of mortality will be:

Low: 0.50 * 100,000 ha * 0.52 carcasses/ha

High: $0.50 * 100,000$ ha* 2.4 carcasses/ha

The reader is reminded that these studies used for this quantification ignore any kills of migrants which, as mentioned earlier, can be substantial. Secondary poisoning of scavengers is also not included nor is delayed mortality or reproductive effects. It will be assumed that all mortality is on adult birds and very rough estimates of the number of failed nests will also be made.

\section{Which species are killed by pesticides?}

A total of 50 agricultural field studies with demonstrated avian mortality ${ }^{6}$ were tallied in order to identify which species are most often killed in the course of pesticide applications. The sample of studies was fairly broadly based, both in terms of the crops sampled (table 4) and the locations of the fields (table 5). The number of studies in which species were found dead is given in table 6. Not all of these species are relevant to Canadian conditions but they were left there because they can provide information on ecologically equivalent species.

\footnotetext{
${ }^{6}$ Most of these studies are proprietary industry studies submitted to the U.S. government. Those dealing with spray applications (29 studies) were reviewed in Mineau 2002. A sample of studies on granular formulations (21 studies) was similarly obtained and reviewed.
} 
Table 4. The number of study-crop combinations for which mortality was detected and which were used in the species tally presented in table 3.

\begin{tabular}{|l|r|}
\hline Crop & $\begin{array}{l}\text { No. } \\
\text { studies }\end{array}$ \\
\hline alfalfa & 6 \\
\hline apple & 4 \\
\hline bare field & 1 \\
\hline barley & 1 \\
\hline canola & 2 \\
\hline carrots & 2 \\
\hline citrus & 4 \\
\hline corn & 13 \\
\hline cotton & 6 \\
\hline potatoes & 6 \\
\hline rangeland & 2 \\
\hline sunflower & 1 \\
\hline wheat & 2 \\
\hline
\end{tabular}

Table 5. Geographical representation of the studies for which mortality was detected and which were used in the species tally presented in table 3.

\begin{tabular}{|l|r|}
\hline State/province & $\begin{array}{l}\text { No. } \\
\text { studies }\end{array}$ \\
\hline Alabama & 1 \\
\hline Arizona & 3 \\
\hline California & 1 \\
\hline Colorado & 1 \\
\hline Delaware & 3 \\
\hline Florida & 3 \\
\hline Idaho & 1 \\
\hline Illinois & 3 \\
\hline lowa & 2 \\
\hline Kansas & 1 \\
\hline Manitoba & 1 \\
\hline Maryland & 2 \\
\hline Michigan & 1 \\
\hline Nebraska & 1 \\
\hline New Jersey & 1 \\
\hline New Mexico & 1 \\
\hline North Carolina & 1 \\
\hline North Dakota & 2 \\
\hline Oklahoma & 1 \\
\hline Pennsylvania & 2 \\
\hline Saskatchewan & 6 \\
\hline Texas & 1 \\
\hline Virginia & \\
\hline
\end{tabular}




\begin{tabular}{|l|r|}
\hline Washington & 5 \\
\hline Wisconsin & 2 \\
\hline Wyoming & 2 \\
\hline
\end{tabular}

Table 6a, b. Species found dead in the studies tallied in tables 1 and 2 and the number of studies that reported kills of that species. Species are ordered by frequency of occurrence - the number of studies in which one or more of the given species was found dead (a) and taxonomic order (b).

a)

\begin{tabular}{|c|c|}
\hline Species & $\begin{array}{l}\text { TOTAL } \\
\text { MENTIONS }\end{array}$ \\
\hline mourning dove & 22 \\
\hline American robin & 14 \\
\hline house sparrow & 14 \\
\hline brown-headed cowbird & 12 \\
\hline horned Lark & 12 \\
\hline red-winged blackbird & 12 \\
\hline $\begin{array}{l}\text { meadowlark (eastern \& } \\
\text { western) }\end{array}$ & 11 \\
\hline common grackle & 10 \\
\hline chipping sparrow & 9 \\
\hline European starling & 9 \\
\hline northern bobwhite & 9 \\
\hline northern cardinal & 9 \\
\hline savannah sparrow & 8 \\
\hline blue jay & 7 \\
\hline indigo bunting & 7 \\
\hline vesper sparrow & 7 \\
\hline brown thrasher & 6 \\
\hline killdeer & 6 \\
\hline ring-necked pheasant & 6 \\
\hline gray catbird & 5 \\
\hline eastern bluebird & 4 \\
\hline house wren & 4 \\
\hline mallard & 4 \\
\hline northern flicker & 4 \\
\hline northern mockingbird & 4 \\
\hline white-crowned sparrow & 4 \\
\hline American crow & 3 \\
\hline American goldfinch & 3 \\
\hline American kestrel & 3 \\
\hline black-billed magpie & 3 \\
\hline California quail & 3 \\
\hline common ground dove & 3 \\
\hline downy woodpecker & 3 \\
\hline greater sage grouse & 3 \\
\hline
\end{tabular}




\begin{tabular}{|c|c|}
\hline great-tailed grackle & 3 \\
\hline lark sparrow & 3 \\
\hline northern harrier & 3 \\
\hline American pipit & 2 \\
\hline Brewer's blackbird & 2 \\
\hline Carolina wren & 2 \\
\hline cedar waxwing & 2 \\
\hline dickcissel & 2 \\
\hline eastern kingbird & 2 \\
\hline eastern towhee & 2 \\
\hline Gambel's quail & 2 \\
\hline grasshopper sparrow & 2 \\
\hline gray partridge & 2 \\
\hline house finch & 2 \\
\hline rock pigeon & 2 \\
\hline rose-breated grosbeak & 2 \\
\hline ruffed grouse & 2 \\
\hline song sparrow & 2 \\
\hline western kingbird & 2 \\
\hline wood thrush & 2 \\
\hline Abert's towhee & 1 \\
\hline American coot & 1 \\
\hline Baltimore oriole & 1 \\
\hline bank swallow & 1 \\
\hline barn swallow & 1 \\
\hline black-bellied whistling ducks & 1 \\
\hline black-capped chickadee & 1 \\
\hline blue grosbeak & 1 \\
\hline boat-tailed grackle & 1 \\
\hline bobolink & 1 \\
\hline burrowing owl & 1 \\
\hline Canada goose & 1 \\
\hline Carolina chickadee & 1 \\
\hline Cassin's sparrow & 1 \\
\hline chestnut-collared longspur & 1 \\
\hline cinnamon teal & 1 \\
\hline clay-coloured sparrow & 1 \\
\hline common nighthawk & 1 \\
\hline dark-eyed junco & 1 \\
\hline Eurasian Tree sparrow & 1 \\
\hline ferrugineous pigmy owl & 1 \\
\hline field sparrow & 1 \\
\hline fish crow & 1 \\
\hline fox sparrow & 1 \\
\hline Franklin's gull & 1 \\
\hline golden-crowned kinglet & 1 \\
\hline greater roadrunner & 1 \\
\hline
\end{tabular}




\begin{tabular}{|l|r|}
\hline greater white-fronted goose & 1 \\
\hline Harris's sparrow & 1 \\
\hline lapland longspur & 1 \\
\hline lark bunting & 1 \\
\hline laughing gull & 1 \\
\hline lazuli bunting & 1 \\
\hline least sandpiper & 1 \\
\hline LeConte's sparrow & 1 \\
\hline lesser nighthawk & 1 \\
\hline loggerhead shrike & 1 \\
\hline long-billed dowitchers & 1 \\
\hline mottled ducks & 1 \\
\hline northern pintail & 1 \\
\hline orange-crowned warbler & 1 \\
\hline rusty blackbird & 1 \\
\hline scarlet tanager & 1 \\
\hline semipalmated sandpiper & 1 \\
\hline short-eared owl & 1 \\
\hline sora rail & 1 \\
\hline spotted sandpiper & 1 \\
\hline Sprague's pipit & 1 \\
\hline Steller's Jay & 1 \\
\hline summer tanager & 1 \\
\hline swallow (unidentified) & 1 \\
\hline swamp sparrow & 1 \\
\hline tree swallow & 1 \\
\hline western tanager & 1 \\
\hline white-throated sparrow & 1 \\
\hline white-winged dove & 1 \\
\hline yellow-breasted chat & 1 \\
\hline yellow-headed blackbird & 1 \\
\hline yellow-rumped warbler & 1 \\
\hline
\end{tabular}

b)

\begin{tabular}{|l|r|}
\hline Species & $\begin{array}{l}\text { TOTAL } \\
\text { MENTIONS }\end{array}$ \\
\hline black-bellied whistling duck & 1 \\
\hline greater white-fronted goose & 1 \\
\hline Canada goose & 1 \\
\hline mallard & 4 \\
\hline mottled ducks & 1 \\
\hline cinnamon teal & 1 \\
\hline northern pintail & 1 \\
\hline gray partridge & 2 \\
\hline ring-necked pheasant & 6 \\
\hline ruffed grouse & 2 \\
\hline
\end{tabular}




\begin{tabular}{|l|r|}
\hline greater sage grouse & 3 \\
\hline California quail & 3 \\
\hline Gambel's quail & 2 \\
\hline northern bobwhite & 9 \\
\hline northern harrier & 3 \\
\hline American kestrel & 3 \\
\hline sora rail & 1 \\
\hline American coot & 1 \\
\hline killdeer & 6 \\
\hline spotted sandpiper & 1 \\
\hline semipalmated sandpiper & 1 \\
\hline least sandpiper & 1 \\
\hline long-billed dowitchers & 1 \\
\hline laughing gull & 1 \\
\hline Franklin's gull & 1 \\
\hline rock pigeon & 2 \\
\hline white-winged dove & 1 \\
\hline mourning dove & 22 \\
\hline common ground dove & 3 \\
\hline greater roadrunner & 1 \\
\hline ferrugineous pigmy owl & 1 \\
\hline burrowing owl & 1 \\
\hline short-eared owl & 1 \\
\hline lesser nighthawk & 1 \\
\hline common nighthawk & 1 \\
\hline downy woodpecker & 1 \\
\hline northern flicker & 1 \\
\hline western kingbird & 1 \\
\hline eastern kingbird & 1 \\
\hline loggerhead shrike & 1 \\
\hline Steller's Jay & 3 \\
\hline blue jay & 2 \\
\hline black-billed magpie & 1 \\
\hline American crow & 1 \\
\hline fish crow & 1 \\
\hline horned Lark & 1 \\
\hline tree swallow & 1 \\
\hline bank swallow & 1 \\
\hline barn swallow & 1 \\
\hline swallow (unidentified) & 1 \\
\hline Carolina chickadee & 1 \\
\hline black-capped chickadee & 1 \\
\hline Carolina wren & 1 \\
\hline house wren & 1 \\
\hline golden-crowned kinglet & 1 \\
\hline eastern bluebird & 1 \\
\hline wood thrush & 1 \\
\hline & 1 \\
\hline
\end{tabular}




\begin{tabular}{|c|c|}
\hline American robin & 14 \\
\hline gray catbird & 5 \\
\hline northern mockingbird & 4 \\
\hline brown thrasher & 6 \\
\hline European starling & 9 \\
\hline American pipit & 2 \\
\hline Sprague's pipit & 1 \\
\hline cedar waxwing & $\overline{2}$ \\
\hline orange-crowned warbler & 1 \\
\hline yellow-rumped warbler & 1 \\
\hline yellow-breasted chat & 1 \\
\hline summer tanager & 1 \\
\hline scarlet tanager & 1 \\
\hline western tanager & 1 \\
\hline eastern towhee & 2 \\
\hline Abert's towhee & 1 \\
\hline Cassin's sparrow & 1 \\
\hline chipping sparrow & 9 \\
\hline clay-coloured sparrow & 1 \\
\hline field sparrow & 1 \\
\hline vesper sparrow & 7 \\
\hline lark sparrow & 3 \\
\hline lark bunting & 1 \\
\hline savannah sparrow & 8 \\
\hline grasshopper sparrow & 2 \\
\hline LeConte's sparrow & 1 \\
\hline fox sparrow & 1 \\
\hline song sparrow & 2 \\
\hline swamp sparrow & 1 \\
\hline white-throated sparrow & 1 \\
\hline Harris's sparrow & 1 \\
\hline white-crowned sparrow & 4 \\
\hline dark-eyed junco & 1 \\
\hline lapland longspur & 1 \\
\hline chestnut-collared longspur & 1 \\
\hline northern cardinal & 9 \\
\hline rose-breated grosbeak & 2 \\
\hline blue grosbeak & 1 \\
\hline lazuli bunting & 1 \\
\hline indigo bunting & 7 \\
\hline dickcissel & 2 \\
\hline bobolink & 1 \\
\hline red-winged blackbird & 12 \\
\hline $\begin{array}{l}\text { meadowlark (eastern \& } \\
\text { western) }\end{array}$ & 11 \\
\hline yellow-headed blackbird & 1 \\
\hline rusty blackbird & 1 \\
\hline Brewer's blackbird & 2 \\
\hline
\end{tabular}




\begin{tabular}{|l|r|}
\hline common grackle & 10 \\
\hline boat-tailed grackle & 1 \\
\hline great-tailed grackle & 3 \\
\hline brown-headed cowbird & 12 \\
\hline Baltimore oriole & 1 \\
\hline house finch & 2 \\
\hline American goldfinch & 3 \\
\hline house sparrow & 14 \\
\hline Eurasian Tree sparrow & 1 \\
\hline
\end{tabular}

Species most frequently implicated in kills are those that are cosmopolitan, closely associated with agriculture and reasonably common; e.g. mourning doves, several sparrows, horned larks and meadowlarks, robins, house sparrows and several blackbird species. However, the sheer diversity of birds potentially killed by pesticides is impressive and suggests that toxicological or ecological susceptibility are less important than being simply in the wrong place at the wrong time. These studies were all carried out in the breeding season and underestimate the impact of pesticide use on migrant species or wintering species. Kills during the breeding season are more relevant to Canadian conditions because of the limited use of pesticides outside the breeding season. A fuller account of species killed by pesticides can be found in the incident record (http://www.abcbirds.org/abcprograms/policy/pesticides/aims/aims/index.cfm). The list also does not include species that are preferentially killed by pesticides on nonagricultural sites; e.g. golf courses and other turf areas. A number of waterfowl species fall in this group. Finally, birds of prey are underrepresented because they typically die well away from application sites. These are not migratory birds so no effort will be made to quantify the kills but Mineau et al. (1999) provided an extensive review of recorded incidents for both Canada and the U.S.

\section{$\underline{\text { Results }}$}

\section{First approach}

Based on US pesticide application data, the following are estimated kills for Canada assuming that each censused crop is grown with the least risk to birds shown by any US State, an average risk to birds based on the weighted average of the 50 conterminous US States or the maximum risk to birds based on the worst State profile for each individual crop. Details for each crop are given in Appendix 1. High and low estimates based on the two kill rates calculated above are summarised in table 7 below. 
Table 7. Summarised results of predicted incidental take from pesticides. This method assumes that the probability of kill for each commodity will be within the range shown for the State by State US analysis of kill probabilities as calculated in Mineau and Whiteside (2006). See appendix 1.

\begin{tabular}{|l|r|r|r|}
\hline & $\begin{array}{l}\text { Canadian } \\
\text { growers grow } \\
\text { the crop like } \\
\text { growers in the } \\
\text { 'best' US State }\end{array}$ & $\begin{array}{l}\text { Canadian } \\
\text { growers grow } \\
\text { the crop like } \\
\text { growers in } \\
\text { the 'worst' US } \\
\text { State }\end{array}$ & $\begin{array}{l}\text { Canadian } \\
\text { growers } \\
\text { grow the } \\
\text { crop like } \\
\text { growers in } \\
\text { an average } \\
\text { US State }\end{array}$ \\
\hline $\begin{array}{l}\text { Low kill rate per } \\
\text { ha }\end{array}$ & 220,160 & $4,473,161$ & 960,011 \\
\hline $\begin{array}{l}\text { High kill rate } \\
\text { per ha }\end{array}$ & $1,016,121$ & $20,645,358$ & $4,430,819$ \\
\hline
\end{tabular}

It is unlikely that we grow each and every crop with less impact on birds than any of the 50 conterminous US States. Assuming that we fall around the average US State in terms of use quantities and avian toxicity of product choice for each commodity, this would place mortality at between 0.96 and 4.4 million birds annually under current (2000-2003) conditions.

It is possible to parse out the expected mortality on a province by province basis based on crop data for each province. Table 7 shows the 6 kill estimates for each province and table 8 shows what percentage of the total Canadian kill each province represents under the same assumptions. 
Table 6. The estimated kill by province under varying assumptions of commodity-specific risk to bird (based on the 50 conterminous US States) and a low and high mortality rate as detailed above. (Numbers will not add up exactly with the Canadian totals shown above because of rounding errors)

\begin{tabular}{|c|c|c|c|c|c|c|c|}
\hline $\begin{array}{l}\text { COMMODITY- } \\
\text { SPECIFIC RISK TO } \\
\text { BIRDS } \\
\text { MORTALITY } \\
\text { ESTIMATE PER } \\
\text { HECTARE } \\
\end{array}$ & MIN & & LOW & LOW & $\mathrm{HIGH}$ & $\mathrm{HIGH}$ & $\mathrm{HIGH}$ \\
\hline Newfoundland & & 154 & 1845 & 849 & 713 & 8515 & 3920 \\
\hline Prince Edward Island & & 815 & 44286 & 17242 & 3763 & 204398 & 79579 \\
\hline Nova Scotia & & 2963 & 32168 & 14538 & 13674 & 148469 & 67099 \\
\hline New Brunswick & & 1649 & 36887 & 16064 & 7611 & 170247 & 74140 \\
\hline Québec & & 7000 & 265193 & 57665 & 32309 & 1223966 & 266145 \\
\hline Ontario & & 8182 & 635847 & 95105 & 37764 & 2934679 & 438944 \\
\hline Manitoba & & 21709 & 567545 & 95500 & 100197 & 2619440 & 440767 \\
\hline Saskatchewan & & 128329 & 1761299 & 443756 & 592286 & 8129072 & 2048106 \\
\hline Alberta & & 44383 & 1027433 & 195321 & 204846 & 4742001 & 901483 \\
\hline British Columbia & & 4811 & 100211 & 23692 & 22206 & 462511 & 109347 \\
\hline TOTAI & & 219997 & 4472715 & 959731 & 1015369 & 20643299 & 4429530 \\
\hline
\end{tabular}

Table 7. The percentage of the total Canadian kill by province under varying assumptions of commodity-specific risk to bird (based on the 50 conterminous US states) and a low and high mortality rate as detailed above.

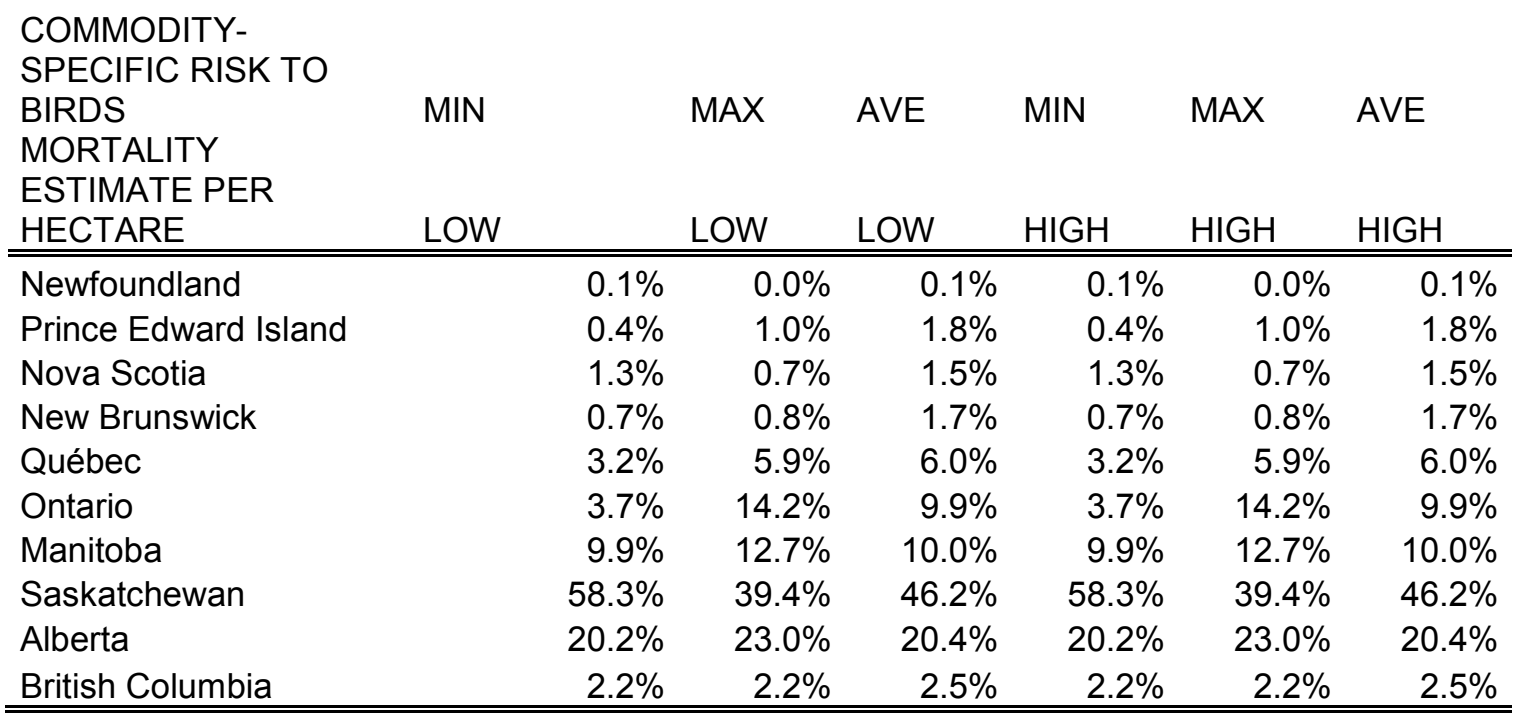

Saskatchewan accounts for approximately half of the total estimated bird mortality, followed by Alberta and Manitoba. The prairies represent most of our area under crop and even if pesticide use in cereal or oilseed crops is less on a per 
hectare basis than in fruit and vegetable crops, to have this large of an area under crop means that a poorly chosen insecticide can have a major impact on birds.

In comparison, the cumulative number of cropped hectares over which avian mortality was likely, was a little over 6 million hectares in the U.S. (Mineau and Whiteside 2006). Using the range of kill rates documented above, this would mean an annual U.S. mortality of 3.16 to 14.6 million birds. This is a substantial reduction from just a few years earlier (1994-1998) when mortality was predicted on a little over 17 million ha. Using the same kill factors, the annual incidental take then would have been estimated at 8.88 to 41.0 million birds annually - approaching the 67 million estimated by Pimentel (1992). Because of restrictions imposed on the most toxic products, the number of 'hectares at lethal risk' has been dropping for most crops $^{7}$. Again, these kill estimates are based solely on those species breeding in heavily agricultural landscapes and frequenting cropped fields - migrants are not included in this calculation. Also unaccounted are seed treatments, some of which could represent important sources of mortality as well as the few herbicides and most rodenticides which have the potential to kill birds.

\section{Second approach}

Based on a simplified field model and the incomplete list of pesticide sales information, the estimated incidental takes were compiled by active ingredient and presumed modal application rate (Table 8). Model results (in the form of predicted number of hectares sustaining mortality per province/territory) are given in appendix 2. The mortality estimates given here assume, as above, that when mortality occurs, there will be a loss of 0.52-2.4 adult individuals per ha.

Table 8. Predicted incidental take based on incomplete characterisation of insecticide sales data for Canada excluding Québec and Saskatchewan. Pesticides are ranked in decreasing order from most to least damaging to birds as measured by the estimated incidental take of adults.

\begin{tabular}{|l|l|l|l|l|l|}
\hline Pesticide & $\begin{array}{l}\text { Modal } \\
\text { application rate } \\
\text { (g a.i./ha) }\end{array}$ & $\begin{array}{l}\text { Avian } \\
\text { HD5 } \\
\text { mg/kg } \\
\text { bw }\end{array}$ & $\begin{array}{l}\text { Predicted risk of } \\
\text { mortality based } \\
\text { on simple } \\
\text { toxicity model }\end{array}$ & $\begin{array}{l}\text { Estimated } \\
\text { incidental } \\
\text { take at low } \\
\text { kill rate }\end{array}$ & $\begin{array}{l}\text { Estimated } \\
\text { incidental } \\
\text { take at high } \\
\text { kill rate }\end{array}$ \\
\hline Chlorpyrifos & 576 & 3.76 & 0.15 & 35054 & 161787 \\
\hline Diazinon & 550 & 0.59 & 0.49 & 28909 & 133424 \\
\hline Carbofuran & 528 & 0.21 & 0.71 & 14144 & 65279 \\
\hline
\end{tabular}

\footnotetext{
${ }^{7}$ These restrictions were the result of U.S. legislation intended to better protect human health, especially children, and not in an effort to reduce avian mortality (Mineau 2006).
} 


\begin{tabular}{|c|c|c|c|c|c|}
\hline Terbufos & 1200 & 0.16 & 0.87 & 4649 & 21456 \\
\hline Azinphos-Methyl & 1128 & 2.28 & 0.35 & 3813 & 17601 \\
\hline Phorate & 3320 & 0.34 & 0.90 & 3561 & 16434 \\
\hline Phosmet & 1475 & 1.24 & 0.55 & 3431 & 15834 \\
\hline Dimethoate & 312 & 5.78 & 0.06 & 2897 & 13369 \\
\hline Methamidophos & 600 & 1.70 & 0.28 & 2406 & 11103 \\
\hline Imidacloprid & 60 & 8.43 & 0.01 & 1762 & 8132 \\
\hline Trichlorfon & 1200 & 13.36 & 0.10 & 1536 & 7091 \\
\hline Carbaryl & 2500 & 30.05 & 0.09 & 1520 & 7017 \\
\hline Endosulfan & 550 & 9.53 & 0.07 & 1423 & 6567 \\
\hline Naled & 950 & 1.72 & 0.37 & 619 & 2855 \\
\hline Methomyl & 870 & 8.46 & 0.11 & 480 & 2213 \\
\hline Acephate & 694 & 18.52 & 0.05 & 407 & 1876 \\
\hline Malathion & 875 & 139.10 & 0.01 & 168 & 776 \\
\hline Pirimicarb & 567 & 6.78 & 0.09 & 127 & 584 \\
\hline Oxamyl & 2244 & 0.78 & 0.73 & 121 & 558 \\
\hline Phosalone & 1000 & 106.27 & 0.01 & 31 & 141 \\
\hline Dicofol & 638 & 72.37 & 0.01 & 11 & 50 \\
\hline $\begin{array}{l}\text { Formetanate } \\
\text { Hydrochloride }\end{array}$ & 1290 & 8.77 & 0.15 & 3 & 13 \\
\hline Amitraz & 850 & 41.83 & 0.03 & 0 & 1 \\
\hline Acetamiprid & 50 & 20.91 & 0.00 & 0 & 0 \\
\hline Pyridaben & 213 & 279.50 & 0.00 & 0 & 0 \\
\hline Tefluthrin & 120 & 178.63 & 0.00 & 0 & 0 \\
\hline Tebufenozide & 144 & 249.71 & 0.00 & 0 & 0 \\
\hline Spinosad & 87 & 170.00 & 0.00 & 0 & 0 \\
\hline
\end{tabular}




\begin{tabular}{|l|r|r|r|r|r|}
\hline Pymetrozine & 97 & 208.12 & 0.00 & 0 & 0 \\
\hline Clofentezine & 200 & 493.59 & 0.00 & 0 & 0 \\
\hline Abamectin & 17 & 42.80 & 0.00 & 0 & 0 \\
\hline $\begin{array}{l}\text { Cyhalothrin- } \\
\text { Lambda }\end{array}$ & 89 & 428.14 & 0.00 & 0 & 0 \\
\hline Fenbutatin Oxide & 38 & 291.52 & 0.00 & 0 & 0 \\
\hline Cypermethrin & 70 & 579.15 & 0.00 & 0 & 0 \\
\hline Deltamethrin & 10 & 97.09 & 0.00 & 0 & 0 \\
\hline Permethrin & 106 & 3127.00 & & & \\
& & & & & \\
\hline TOTAL & & & & $\mathbf{1 0 7 0 6 9}$ & $\mathbf{4 9 4 1 6 3}$ \\
\hline
\end{tabular}

These estimates are lower than those obtained using the first method, in part because of the incomplete and dated sales and use data available. Given that Saskatchewan accounted for approximately half of the total estimated mortality according to our first estimation method, the absence of any use data for that province is a clear problem. Estimates derived from this second approach therefore lack credibility. They are kept here because they do indicate which pesticides are likely causing the most mortality.

\section{Associated loss of nesting opportunities}

Incidental killing of adult birds by pesticides could take place at any point in the breeding season. Granular pesticides (or seed treatments not tallied here) would kill breeding individuals early in the season. Insecticide use is typically carried out at any point between seedling emergence up to a few weeks before harvest.

A very rough assessment of the number of lost nests is possible if we are willing to make a number of simplifying assumptions, viz.

1) Paired individuals from the same nest are likely to be killed together; there will be half the number of nests at risk than there are affected individuals;

2) Most agricultural species will have one re-nesting attempt; 
3) An average success rate for most agricultural species will be one fledged young per nesting attempt;

4) Half of the kills will take place at the time when a nest is present and therefore result in the loss of the nest;

We can therefore easily envision the loss of $50 \%$ more individuals as a result of lost nesting opportunity. These assumptions are not very extreme and the additional breeding deficit is likely higher than this.

\section{Discussion}

Population relevance of the mortality

Many of the species most affected are well distributed farmland species. However, several of them (e.g. Horned lark, Vesper sparrow) are already in clear decline over most or all of their range. A link between pesticide use and regional population levels in the prairies has already been made (Mineau et al. 2005). Other forthcoming analyses (Mineau, unpublished) suggest that the direct acute and subacute toxicity of pesticides has significantly contributed to the decline of grassland/farmland bird species in North America.

The birds that are killed are 'valuable members' of the population. They have survived at least two full migrations, have returned to the breeding grounds and have successfully defended a territory. Therefore, any impact on this cohort is proportionately higher than similar estimates derived for other sources of mortality.

\section{Mitigation}

Mitigation of kills is relatively easy. Products that kill birds readily and reliably are well known and have been so identified. In most cases, substitution products of lower toxicity to birds already exist. Regulatory inaction is the only impediment to a reduction of the direct incidental take. Chronic and indirect effects will be slightly harder to mitigate although here also, much information exists on which products carry the highest risk.

\section{References}

Brimble, S., P. Bacchus and P.-Y. Caux. 2005. Pesticide utilization in Canada: A compilation of current sales and use data. Environment Canada Unpublished report. $144 \mathrm{pp}$. 
EFSA. 2008. Scientific Opinion of the Panel on Plant protection products and their residues on a request from the EFSA PRAPeR Unit on risk assessment for birds and mammals. The EFSA Journal (2008) 734, 1-181.

FMC (FMC Corporation). 1989a. Effects of Furadan 4F on birds associated with Nebraska and Texas/New Mexico corn fields. [C.D. Jorgensen, R.C. Whitmore, G.M. Booth, M.W. Carter, and H.D. Smith] Unpublished report. 751 pp.

FMC (FMC Corporation). 1989b. Effects of Furadan 4F on birds associated with Kansas and Oklahoma alfalfa fields. [G.M. Booth, L.B. Best, M.W. Carter, and C.D. Jorgensen] Unpublished report. 810 pp.

Mineau, P. 1993. The hazard of carbofuran to birds and other vertebrate wildlife. Technical Report Series. No. 177. Environment Canada, Canadian Wildlife Service, Ottawa. XXii+96 pp.

Mineau, P., M.R. Fletcher, L.C. Glazer, N.J. Thomas, C. Brassard, L.K. Wilson, J.E. Elliott, L.A. Lyon, C.J. Henny, T. Bollinger, and S.L. Porter. 1999 Poisoning of raptors with organophosphorous and carbamates pesticides with emphasis on Canada, U.S. and U.K. J. Raptor Research 33(1):1-37.

Mineau, P., A. Baril, B.T. Collins, J. Duffe, G. Joerman, and R. Luttik. 2001. Reference values for comparing the acute toxicity of pesticides to birds. Reviews of Environmental Contamination and Toxicology. 170:13-74.

Mineau, P. 2002. Estimating the probability of bird mortality from pesticide sprays on the basis of the field study record. Environmental Toxicology and Chemistry. 24(7):1497-1506.

Mineau, P. 2003. Avian species. In: Plimmer, J.R., D.W. Gammon, and N.N. Ragsdale (Eds.). Encyclopedia of Agrochemicals. Vol. 1. Wiley Interscience. pp. 129-156.

Mineau, P. 2004. Birds and pesticides: are pesticide regulatory decisions consistent with the protection afforded migratory bird species under the Migratory Bird Treaty Act? The William and Mary Environmental Law and Policy Review. 28(2): 313-338.

Mineau P. 2005. Direct losses of birds to pesticides - Beginnings of a quantification. IN Bird Conservation Implementation and Integration in the Americas: Proceedings of the Third International Partners in Flight Conference 2002 (C.J. Ralph and T.D. Rich, eds.), U.S.D.A. Forest Service, GTR-PSW-191, Albany, CA; Vol. 2, pp. 10651070.

Mineau, P., C.M. Downes, D.A. Kirk, E. Bayne, \& M. Csizy. 2005. Patterns of bird species abundance in relation to granular insecticide use in the Canadian prairies. Ecoscience 12(2):267-278. 
Mineau, P. 2005. A review and analysis of study endpoints relevant to the assessment of 'long term' pesticide toxicity in avian and mammalian wildlife. Ecotoxicology. 14(8):775-799.

Mineau, P. and M. Whiteside. 2006. The lethal risk to birds from insecticide use in the U.S. - a spatial and temporal analysis. Environmental Toxicology and Chemistry. 25(5):1214-1222.

Mineau, P., T. Dawson, M. Whiteside, C. Morrison, K. Harding, L. Singh, T. Längle, and D.A.R. McQueen. Environmental Risk-based Standards for Pesticide Use in Canada. National Agri-Environmental Standards Initiative Synthesis Report.

Environment Canada, In Press (Jan 08).

Pimentel, D., H. Acquay, M. Biltonen, P. Rice, M. Silva, J. Nelson, V. Lipner, S. Giordano, A. Horowitz, and M. D'Amore. 1992. Environmental and economic costs of pesticide use. BioScience 42:750-760.

Ruben N. Lubowski, Marlow Vesterby, Shawn Bucholtz, Alba Baez, and Michael J. Roberts. Major Uses of Land in the United States, 2002. Economic Information Bulletin No. (EIB-14) 54 pp, May 2006 http://www.ers.usda.gov/Publications/ElB14/

Smallwood, K.S. 2007. Estimating Wind Turbine-Caused Bird Mortality. J. Wildl. Manage. 71(8):2781-2791.

Smith, G.K. 2006. Risks to birds from pesticide-treated seed and the possible role of ultraviolet reflection in seed colour preferences and repellent strategies. MSc. Thesis (unpublished). Carleton University. Ottawa, Ontario. 175 p.

Statistic Canada. 2009. Agriculture overview, Canada and the provinces \& Farm data and farm operator data tables. 2006 Census of Agriculture. http://www.statcan.gc.ca/pub/95-629-x/2007000/4182409-eng.htm , accessed March-April 2009. 
Appendix 1.

\begin{tabular}{|c|c|c|c|c|c|c|}
\hline Crop & $\begin{array}{l}\text { No. of } \\
\text { States } \\
\text { surveyed }\end{array}$ & $\begin{array}{l}\text { Year of } \\
\text { survey }\end{array}$ & $\begin{array}{l}\text { Canadian } \\
\text { area of crop } \\
\text { (ha) } 2008\end{array}$ & $\begin{array}{l}\text { Min. } \\
\text { number of } \\
\text { ha with } \\
\text { mortality } \\
\text { risk }\end{array}$ & $\begin{array}{l}\text { Max. } \\
\text { number of } \\
\text { ha with } \\
\text { mortality } \\
\text { risk }\end{array}$ & $\begin{array}{l}\text { Ave. number of } \\
\text { ha with mortality } \\
\text { risk if pesticide } \\
\text { use corresponds } \\
\text { to weighted US } \\
\text { average }\end{array}$ \\
\hline Alfalfa & 47 & 1997 & 5075560 & 25378 & 2913371 & 451725 \\
\hline Apples & 8 & 2001 & 22101 & 2122 & 95300 & 15117 \\
\hline Apricots & 1 & 2001 & 200 & 42 & 42 & 42 \\
\hline Asparagus & 3 & 2002 & 2104 & 688 & 1523 & 1290 \\
\hline Barley & 2 & 2003 & 4039563 & 0 & 20198 & 12119 \\
\hline Beets & 6 & 1997 & 3139 & 9 & 1585 & 421 \\
\hline Blackberries & 1 & 2001 & 1757 & 307 & 307 & 307 \\
\hline Blueberries & 4 & 2001 & 52608 & 15940 & 117737 & 75387 \\
\hline Broccoli & 1 & 2002 & 4489 & 3228 & 3228 & 3228 \\
\hline $\begin{array}{l}\text { Brussel } \\
\text { sprouts }\end{array}$ & 1 & 2000 & 561 & 1337 & 1337 & 1337 \\
\hline Cabbage & 9 & 2002 & 5490 & 0 & 2300 & 994 \\
\hline Canola & 8 & 1997 & 5164038 & 129101 & 1962334 & 170413 \\
\hline Carrots & 4 & 2002 & 9857 & 0 & 9275 & 315 \\
\hline Cauliflower & 1 & 2002 & 2144 & 845 & 845 & 845 \\
\hline Celery & 1 & 2002 & 908 & 298 & 298 & 298 \\
\hline Cherries & 5 & 2001 & 2945 & 241 & 5375 & 1358 \\
\hline Corn & 16 & 2003 & 1392100 & 0 & 318791 & 62645 \\
\hline Cranberries & 5 & 1997 & 3415 & 2326 & 8794 & 7236 \\
\hline Cucumbers & 6 & 2002 & 2903 & 0 & 1527 & 203 \\
\hline Dry beans & 17 & 1997 & 184842 & 0 & 56192 & 10536 \\
\hline Dry peas & 5 & 1997 & 1915783 & 181999 & 1383195 & 568988 \\
\hline Eggplant & 2 & 2000 & 5257 & 400 & 1130 & 904 \\
\hline Flax & 3 & 1997 & 807975 & 0 & 0 & 0 \\
\hline Grapes & 5 & 2001 & 12164 & 36 & 8770 & 511 \\
\hline Green beans & 9 & 2002 & 10998 & 0 & 5059 & 1716 \\
\hline Green onions & 2 & 1997 & 1008 & 216 & 274 & 235 \\
\hline Green peas & 4 & 2002 & 16831 & 0 & 572 & 135 \\
\hline Lettuce & 2 & 2002 & 3911 & 1072 & 2835 & 2405 \\
\hline Oats & 37 & 1997 & 2063612 & 0 & 70163 & 10318 \\
\hline Onions & 6 & 2002 & 5823 & 1473 & 8746 & 4041 \\
\hline Other hay & 33 & 1997 & 2893649 & 0 & 2894 & 1447 \\
\hline Peaches & 5 & 2001 & 3802 & 2365 & 24504 & 6779 \\
\hline Pears & 3 & 2001 & 1486 & 499 & 1051 & 744 \\
\hline Plums/prunes & 1 & 2001 & 751 & 177 & 177 & 177 \\
\hline Potatoes & 10 & 2003 & 162515 & 488 & 258561 & 103522 \\
\hline Pumpkins & 3 & 2002 & 3765 & 0 & 184 & 49 \\
\hline Radishes & 7 & 1997 & 682 & 1 & 839 & 239 \\
\hline Raspberries & 2 & 2001 & 3635 & 1327 & 1796 & 1661 \\
\hline Rye & 15 & 1997 & 215185 & 0 & 0 & 0 \\
\hline Safflower & 2 & 1997 & 91371 & 26589 & 26589 & 26589 \\
\hline
\end{tabular}




\begin{tabular}{|l|r|r|r|r|r|r|}
\hline Seed crops & 21 & 1997 & 412985 & 18584 & 44602 & 41711 \\
\hline Sod & 23 & 1997 & 27960 & 28 & 1454 & 1398 \\
\hline Soybeans & 8 & 2002 & 1202098 & 0 & 580613 & 22840 \\
\hline Spinach & 2 & 2002 & 711 & 0 & 171 & 152 \\
\hline Squash & 6 & 2002 & 9571 & 0 & 6374 & 2728 \\
\hline Strawberries & 3 & 2002 & 5204 & 1093 & 3471 & 3148 \\
\hline Sugar beets & 9 & 2000 & 19488 & 2865 & 16565 & 12511 \\
\hline Sugarcane & 3 & 1997 & & 0 & 0 & 0 \\
\hline Sunflowers & 9 & 1997 & 85402 & 1281 & 62941 & 6149 \\
\hline Sweet corn & 12 & 2002 & 30229 & 0 & 49455 & 7739 \\
\hline $\begin{array}{l}\text { Sweet } \\
\text { peppers }\end{array}$ & 4 & 2002 & 2458 & 22 & 3380 & 1428 \\
\hline Tobacco & 16 & 1997 & 12918 & 1008 & 22322 & 10670 \\
\hline Tomatoes & 5 & 2002 & 9010 & 0 & 18849 & 1667 \\
\hline Wheat & 3 & 2002 & 9881991 & 0 & 474336 & 187758 \\
\hline
\end{tabular}

Notes: The following crop combinations and substitutions were made in order to make the Census of Agriculture data to conform with USDA data from Mineau and Whiteside (2006).

- Barley includes mixed grain and buckwheat

- Beets includes rutabagas and turnip

- 'Other berries' assumed to be similar to blackberries

- Blueberries includes Saskatoon berries

- Cabbage includes Chinese cabbage

- Canola includes mustard seed

- Cherries includes both sweet and sour cherries

- Dry peas includes dry lentils and chick peas

- 'Other vegetables' assumed to be most similar to eggplant

- 'Other field crops' assumed to be similar to safflower

- 'Seed crops' includes forage seed, canary seed and caraway

- Squash included zucchini and mixed squash/pumpkin fields

- Wheat includes triticale 


\section{Appendix 2}

\begin{tabular}{|c|c|c|c|c|c|c|c|c|c|c|c|c|c|}
\hline & & & & \multicolumn{10}{|c|}{$\begin{array}{l}\text { Estimated area (ha) over which avian mortality will occur as a result of the insecticide } \\
\text { indicated. }\end{array}$} \\
\hline Pesticide & $\begin{array}{l}\text { Modal } \\
\text { application } \\
\text { rate }(\mathrm{g} \\
\text { a.i./ha) }\end{array}$ & $\begin{array}{l}\text { Avian } \\
\text { HD5 } \\
\mathrm{mg} / \mathrm{kg} \\
\text { bw }\end{array}$ & $\begin{array}{l}\text { Predicted } \\
\text { risk of } \\
\text { mortality } \\
\text { based on } \\
\text { simplified } \\
\text { field } \\
\text { model }\end{array}$ & $\begin{array}{l}\text { B.C. } \\
(2003)\end{array}$ & $\begin{array}{l}\text { Alta. } \\
(1998)\end{array}$ & $\begin{array}{l}\text { Man. } \\
(2003)\end{array}$ & $\begin{array}{l}\text { Ont. } \\
(2003)\end{array}$ & \begin{tabular}{l|} 
N.B. \\
$(2003)$
\end{tabular} & $\begin{array}{l}\text { N.S. } \\
(2003)\end{array}$ & $\begin{array}{l}\text { P.E.I. } \\
(2002)\end{array}$ & $\begin{array}{l}\text { N.L. } \\
(2003)\end{array}$ & $\begin{array}{l}\text { Y.T. } \\
(1994)\end{array}$ & $\begin{array}{l}\text { N.W.T. } \\
\text { (1995) }\end{array}$ \\
\hline Phorate & 3320 & 0.34 & 0.90 & 0 & 5184 & 647 & 0 & 237 & 779 & 0 & 0 & 0 & 0 \\
\hline Terbufos & 1200 & 0.16 & 0.87 & 2329 & 4860 & 0 & 1255 & 326 & 170 & 0 & 0 & 0 & 0 \\
\hline Oxamyl & 2244 & 0.78 & 0.73 & 229 & 3 & 0 & 0 & 1 & 0 & 0 & 0 & 0 & 0 \\
\hline Carbofuran & 528 & 0.21 & 0.71 & 650 & 8618 & 8313 & 2389 & 1061 & 122 & 6046 & 0 & 0 & 0 \\
\hline Phosmet & 1475 & 1.24 & 0.55 & 778 & 138 & 149 & 4604 & 15 & 914 & 0 & 0 & 0 & 0 \\
\hline Diazinon & 550 & 0.59 & 0.49 & 24261 & 3663 & 0 & 3558 & 2950 & 3393 & 0 & 17767 & 1 & 2 \\
\hline Naled & 950 & 1.72 & 0.37 & 555 & 495 & 0 & 124 & 0 & 15 & 0 & 0 & 0 & 0 \\
\hline $\begin{array}{l}\text { Azinphos- } \\
\text { Methyl }\end{array}$ & 1128 & 2.28 & 0.35 & 2019 & 81 & 1091 & 3077 & 986 & 78 & 0 & 0 & 0 & 0 \\
\hline Methamidophos & 600 & 1.7 & 0.28 & 464 & 9 & 197 & 462 & 1367 & 7 & 2121 & 0 & 0 & 0 \\
\hline Chlorpyrifos & 576 & 3.76 & 0.15 & 1217 & 58006 & 5564 & 1432 & 734 & 458 & 0 & 1 & 0 & 0 \\
\hline $\begin{array}{l}\text { Formetanate } \\
\text { Hydrochloride }\end{array}$ & 1290 & 8.77 & 0.15 & 0 & 0 & 0 & 0 & 0 & 5 & 0 & 0 & 0 & 0 \\
\hline Methomyl & 870 & 8.46 & 0.11 & 43 & 56 & 0 & 71 & 742 & 10 & 0 & 0 & 0 & 0 \\
\hline Trichlorfon & 1200 & 13.36 & 0.10 & 0 & 2849 & 0 & 18 & 83 & 3 & 0 & 0 & 0 & 0 \\
\hline Pirimicarb & 567 & 6.78 & 0.09 & 85 & 25 & 0 & 106 & 3 & 24 & 0 & 0 & 0 & 0 \\
\hline Carbaryl & 2500 & 30.05 & 0.09 & 462 & 117 & 1841 & 181 & 71 & 216 & 0 & 35 & 0 & 0 \\
\hline Endosulfan & 550 & 9.53 & 0.07 & 587 & 95 & 226 & 458 & 762 & 48 & 559 & 0 & 0 & 0 \\
\hline Dimethoate & 312 & 5.78 & 0.06 & 859 & 1009 & 931 & 1355 & 305 & 177 & 930 & 5 & 0 & 0 \\
\hline Acephate & 694 & 18.52 & 0.05 & 66 & 17 & 0 & 634 & 12 & 6 & 0 & 0 & 46 & 0 \\
\hline
\end{tabular}




\begin{tabular}{|c|c|c|c|c|c|c|c|c|c|c|c|c|c|}
\hline Amitraz & 850 & 41.83 & 0.03 & 0 & 0 & 0 & 0 & 0 & 0 & 0 & 0 & 0 & 0 \\
\hline Phosalone & 1000 & 106.27 & 0.01 & 25 & 0 & 0 & 24 & 3 & 7 & 0 & 0 & 0 & 0 \\
\hline Dicofol & 638 & 72.37 & 0.01 & 12 & 8 & 0 & 0 & 0 & 0 & 0 & 0 & 0 & 0 \\
\hline Imidacloprid & 60 & 8.43 & 0.01 & 91 & 2 & 1028 & 91 & 1354 & 43 & 779 & 0 & 0 & 0 \\
\hline Malathion & 875 & 139.1 & 0.01 & 49 & 236 & 12 & 16 & 6 & 3 & 0 & 0 & 0 & 0 \\
\hline Acetamiprid & 50 & 20.91 & 0.00 & 0 & 0 & 0 & 0 & 0 & 0 & 0 & 0 & 0 & 0 \\
\hline Pyridaben & 213 & 279.5 & 0.00 & 0 & 0 & 0 & 0 & 0 & 0 & 0 & 0 & 0 & 0 \\
\hline Tefluthrin & 120 & 178.63 & 0.00 & 0 & 0 & 0 & 0 & 0 & 0 & 0 & 0 & 0 & 0 \\
\hline Tebufenozide & 144 & 249.71 & 0.00 & 0 & 0 & 0 & 0 & 0 & 0 & 0 & 0 & 0 & 0 \\
\hline Spinosad & 87 & 170 & 0.00 & 0 & 0 & 0 & 0 & 0 & 0 & 0 & 0 & 0 & 0 \\
\hline Pymetrozine & 96.5 & 208.12 & 0.00 & 0 & 0 & 0 & 0 & 0 & 0 & 0 & 0 & 0 & 0 \\
\hline Clofentezine & 200 & 493.59 & 0.00 & 0 & 0 & 0 & 0 & 0 & 0 & 0 & 0 & 0 & 0 \\
\hline Abamectin & 17 & 42.8 & 0.00 & 0 & 0 & 0 & 0 & 0 & 0 & 0 & 0 & 0 & 0 \\
\hline $\begin{array}{l}\text { Cyhalothrin- } \\
\text { Lambda }\end{array}$ & 89 & 428.14 & 0.00 & 0 & 0 & 0 & 0 & 0 & 0 & 0 & 0 & 0 & 0 \\
\hline $\begin{array}{l}\text { Fenbutatin } \\
\text { Oxide }\end{array}$ & 37.5 & 291.52 & 0.00 & 0 & 0 & 0 & 0 & 0 & 0 & 0 & 0 & 0 & 0 \\
\hline Cypermethrin & 70 & 579.15 & 0.00 & 0 & 0 & 0 & 0 & 0 & 0 & 0 & 0 & 0 & 0 \\
\hline Deltamethrin & 10 & 97.09 & 0.00 & 0 & 0 & 0 & 0 & 0 & 0 & 0 & 0 & 0 & 0 \\
\hline Permethrin & 106 & 3127 & 0.00 & 0 & 0 & 0 & 0 & 0 & 0 & 0 & 0 & 0 & 0 \\
\hline
\end{tabular}




\title{
Bird Casualty Related to Electrocution on Distribution Power Lines, Maintenance of Transmission Power lines and Hydro-power Reservoirs in Canada.
}

\author{
By
}

\section{Jean-Pierre L. Savard}

Environment Canada, Science and Technology Branch, 801-1550 av d'Estimauville, Québec, G1J 0C3.

and

\section{Sébastien Rioux}

Independent researcher, 3474 Boulevard Neilson Apartment 7, Québec, QC, Canada, G1W 2W1

\begin{abstract}
This study provides estimates for three sources related to power industry in Canada, which cause avian mortality: electrocutions, construction and transmission line maintenance, and water reservoirs. Estimates of avian mortality due to electrocution mortality for Canada (number of poles*birds/pole/year) ranged from 160,836 to 801,962 birds annually. Impacts are likely to be greater on raptors, owls, herons and cranes than on smaller, but more highly productive birds. Construction of transmission line likely impacted 2,588,494 nests and transmission line maintenance can possibly affect 388,274 nests each year. Avian mortality related to the construction and maintenance of transmission and distribution power lines is relatively small, and likely does not cause any significant impact at the population level. Operations of large water reservoirs created for hydro-power will rarely affect adults but result in the destruction of eggs in nidifigous species such as waterfowl and shorebirds or altricial species nestlings. An approximate estimate of avian mortality for Quebec due to hydro-power reservoirs gave us 152,162 nests/year, and does not cause a significant impact on a population level for any species. However, reservoirs within the breeding range of the Piping Plover (Charadrius melodus), an endangered species, could affect the local population if highly estimated losses were recurrent every year.
\end{abstract}

\section{INTRODUCTION}

Power industry in Canada causes multiple impacts on bird species, due to electrocutions, construction and transmission line maintenance, establishment and operation of large reservoirs for hydro-power. This impact varies depending on species and habitat.

\section{Electrocution}


Electrocution mortality can have population level impacts in some areas on select species (Rubolini et al. 2001). The most publicized impacts occurred on large raptors such as Golden Eagle, Aquila chrysaetos (Boeker and Dikerson 1975, Benson 1981). While most of the literature about bird electrocutions focused on raptors, many non-raptors and migratory birds were listed as casualties, including waterfowl, herons, gulls, ravens and other passerines (Lasch et al. 2010, Janss 2000, Bevanger 1998, O’Neil 1988, Dexter 1953, Cartron et al. 2005, Manzano-Fischer 2006, Platt 2005, Anderson 1933). The extent of mortality on non-raptors is difficult to assess.

Recent reviews of available information worldwide concluded that reliable estimates of electrocution mortality are generally unavailable, several studies being affected by design and sampling issues (Lehman et al. 2007, 2010). Several factors contribute to electrocution risks (Roig-Soles and Navazo-Lopez 1997, Platt 2005, Lehman 2007) including bird morphology, age and sex (Ferrer et al. 1991, Dawson and Mannan 1995, Janss 2000, Harness and Wilson 2001), pole type, design and configuration (Boeker and Nikerson 1975, Orlendorff et al. 1981, Ferrer et al. 1991, Slater and Smith 2010) habitat and topography (Boeker and Nickerson 1975, Kochert and Orlendoff 1999), season and weather (Benson 1981, Janss and Ferrer 1999a, b, Harness and Wilson 2001, Platt 2005) which make global estimates of electrocution casualties difficult to collect.

Available estimates tend to be localised and biased by lack of information on detectability, and scavenging and crippling rates (Bevanger 1999). In the case of electrocution, detectability is not a major bias because birds tend to die close to the pole. However, scavenging and crippling rates estimates or lack of, can introduce important biases (Lehman et al. 2007). Reliable scavenging estimates are difficult to obtain as the use of surrogate species (i.e. chicken instead of eagles) can greatly bias estimates because small birds can be removed 10-20 time faster than larger birds (Smallwood 2007). Also, scavenging rates vary between seasons and sites (Bevanger 1995, Janss and Ferrer 1999a, b).

To date there is overwhelming evidence that most mortalities related to electrocution involve large birds mostly owls and raptors (Bevanger 1998, Lehman 2001, Platt 2005, Lehman et al. 2007, 2010, Manville II 2005). In Québec, confirmed bird electrocutions are mostly owls and raptors (Table 1). However, other species are electrocuted in larger numbers than raptors (Anderson 1933, Bevanger 1998, Dedon and Colson 1988, Janss and Ferrer 1999a, b, Janss 2000, Platt 2005, Tinto et al. 2010). Lasch et al. (2010) found that $56 \%$ of mortalities were corvids and gulls. Likewise Manzano-Fisher (2006) documented $>50 \%$ birds that were electrocuted were ravens in her study. Platt (2005) in Alberta found $78 \%$ of electrocutions were non-raptors including ducks, gulls, sharp-tailed grouse, ravens, and other passerines. Janss (2000) found 33\% of electrocutions were two species of migratory birds. Two old references provide insight into potentially overlooked causes of electrocution. Dexter (1953) found an electrocuted Northern Oriole (Icterus galbula) that appeared to have shorted out between a wire and a branch. Anderson (1933) recorded roosting Purple Martins (Progne subis) shorting out between wing tips resulting in group electrocutions. 
Bird electrocution has been a continuous preoccupation for power line managers as it often causes power outage. In the United States, it is an important source of mortality for eagles (Harness and Wilson 2001). Several studies have looked at the efficiency of various measures to reduce casualties (Miller et al. 1975, Ledger 1984, Roig-Soles and Navazo-Lopez 1997, Janss and Ferrer 2001, APLIC 2006, Lammers and Collopy 2007, Slater and Smith 2010) but their efficiency is poorly documented (Lehman et al. 2007).

A detailed study in southeast Alberta (Platt 2005) in a 113,400 $\mathrm{km}^{2}$ area yielded, after correcting for the effect of scavengers, losses of 542-2762 raptors, mostly Great Horned Owls (Bubo virginianus) and Red-tailed Hawks (Buteo jamaicensis) over a six week period spanning June-August. However, this study documented 61 non-raptor mortalities (page 30, Appendix D) compared to 33 raptor mortalities, or a ratio of nearly two to one. Thus the non-raptor losses would be about 1084-5524 birds in a 6 week period in the detailed study area which was only a small proportion of prairie Canada. This number may even be greater as removal experiments used large carcasses and likely underestimated removal rates of smaller birds. Because of the lack of standardized study in Canada, it is impossible to evaluate the number of migratory birds electrocuted each year. Because of their size and persistence, casual reporting of electrocution is likely biased towards large birds.

\section{Construction and maintenance}

Transmission line construction, maintenance, and creation of hydro-power reservoirs change a bird habitat during the breeding season, and therefore, may cause inadvertent nest destruction. In most cases it will rarely affect adults but will result in destruction of eggs in nidifugous species, and eggs or nestlings in atricial species. Sometimes, habitat modification and loss of nest may force adults to move to adjacent habitats and render them more vulnerable to predation.

The amount of casualties depends on nest abundance in habitat types destroyed during vegetation clearing for construction or during habitat vegetation maintenance activities. Casualties can be partitioned into two components: a) nests that would be destroyed during clearing of vegetation in construction phase, which is a one-time impact, $b$ ) nests that would be destroyed during maintenance activities, which is a recurring impact.

The main impact of reservoirs is the loss of habitats through inundation (Baxter and Claude 1980). However, fluctuating water levels associated with reservoir exploitation may also flood nests (Wolf 1955, Books 1985). Nest mortality here is partitioned into three components: a) nests that would be destroyed during clearing of vegetation prior to initial flooding; $b$ ) nests that would be flooded during reservoir filling; $c$ ) nests that would be flooded during annual water fluctuations related to reservoir exploitation. The first two are one time effects whereas the third one is a recurring effect leading to cumulative impacts.

To our knowledge, few adequate studies of bird mortality due to power lines have been done in Canada (Platt 2005) to generate credible estimates for each province. Here, we 
coarsely estimate losses due to electrocution, construction and maintenance of transmission and distribution power lines, and grossly estimate IT due to creation and operation of large water hydro-power reservoirs in Canada.

\section{METHODS}

\section{Electrocutions}

As expected, estimates of bird electrocution rates varied wildly between 11 studies compiled (Table 2). When estimates are expressed in number of birds/pole/year, we often obtain minimum and maximum values depending on the study (Table 2). Minimum values are based on confirmed cases of electrocution only whereas maximum values include all birds found (It is often difficult to determine the exact cause of mortality). We derived average values using: 1) the smaller of two values when two estimates were provided and 2) the maximum value. This yielded average estimates of 0.0281 and $0.1401 \mathrm{bird} /$ pole/year respectively (Table 2). However, results from the Alberta study are based on a relatively short period and contrast with other values. If we exclude values from this study, we obtain average estimates of 0.0066 and $0.0797 \mathrm{bird} /$ pole/year (min and max; Table 2). Estimates are usually per year although this is not clearly stated in some studies. The coefficient of variation was quite high (minimum estimate $=172 \%$; maximum $=132 \%$ ) indicating the wide range in estimates. If we exclude the Alberta study, the coefficient of variation drops to 52-97\%. Given differences between minimum and maximum average estimates we derived estimates for both. Also, because of the unique high estimates obtained in the Alberta study compared to other studies, we derived estimates excluding that study.

\section{Transmission line maintenance and construction}

To derive an estimate of casualties in terms of number of nests destroyed during construction or maintenance, we multiply area cleared during the breeding season by nest density at the time of vegetation clearing.

\section{Landbird density estimates}

DesGranges et al. (2003) compiled and derived breeding bird estimates in diverse types of forested habitats in Quebec (Table A1.1). Estimates of breeding densities ranged between 2.73 and 4.64 pairs/ha and averaged 3.78 pairs/ha. One forest type left out of this average is jack pine which averaged 0.82 pairs/ha. Another source of breeding bird density data is the Bird Census database (Kennedy et al. 1999) which covers several areas of Canada. Estimates of breeding densities varied between locations and habitat types from $0.33 \mathrm{birds} / \mathrm{ha}$ in young Englemann spruce/subalpine fir to $11.9 \mathrm{birds} / \mathrm{ha}$ in mid-age bur oak/green ash/Manitoba maple. Estimates for regenerating clearcuts, a typical habitat found under managed power lines, averaged $8.02 \mathrm{birds} / \mathrm{ha}(\mathrm{n}=18 \mathrm{plots})$ or $4.01 \mathrm{pairs} / \mathrm{ha}$ (Table A1.2; Kennedy et al. 1999). 
Yahner et al. (2004) reported breeding densities of 11.4, 5.0 and 3.8 pairs/ha in right of way corridors. Bramble et al. (1994) reported densities of 9.63 to 15.81 pairs/ha for differently managed right of way corridors. Thus there could be large fluctuations in breeding bird densities depending on location, habitat types and years. Breeding bird densities varied between BCR zones from 3.70 pairs/ha in the Northwestern Interior Forest Zone to 7.74/ha in the Northern Pacific Rainforest Zone (Hobson 2011). However, Savard et al. (2000) reported estimates based on spot-mapping results for the coastal forests of British Columbia ranging between 1.25 and 4.64 pairs/ha depending on forest type and age (Table A1.3).

The above density values represent total breeding density estimates and assume that all species breed simultaneously which is not the case. At any one time, it assumes that all species would have nests at the time of vegetation clearing which is not realistic as the breeding season extends from early may for resident species to late June-early July for neotropical migrants, and several species do re-nest when their nests are destroyed early in the season. Also, a small proportion of nests would have already failed at the time of habitat modifications.

Clearing of a given area will likely take only a few days and the number of active nests will vary according to the timing of the clearing. Also, breeding bird densities and predation pressures can vary greatly between years in relation to weather, insect epidemics and other resources abundance. Because of the difficulties of quantifying these factors and their great yearly variability, for the purpose of this exercise, we assume that $30 \%$ of the nests were active in May, $90 \%$ in June and 30\% in July. These proportions vary between areas (north vs south) and years depending on climate conditions. Therefore, we also generate estimates assuming that $100 \%$ of the nests were active each month.

\section{Transmission lines length}

In Quebec, it is estimated that there are about 12,216 km of transmission lines (Tecsul 2009 ) and $219,750 \mathrm{~km}$ in the rest of Canada (Canadian Electricity Association). However, according to Hydro Quebec web site, the number calculated by Tecsul (2009) may not cover the whole province as it is reported that there is $32,000 \mathrm{~km}$ of transmission lines in Quebec. The latter figure will be used in this report yielding a total of 251,750 km of transmission lines in Canada (32,000 km in Quebec and 219,750 km in the rest of Canada). The total length of distribution lines in Canada is $572,370 \mathrm{~km}$.

\section{Reservoirs}

\section{Area covered by reservoirs}

Approximately $14,831 \mathrm{~km}^{2}$ were inundated by the creation of reservoirs in northern Quebec, within the boreal forest Bird Conservation Area (BCR 8; Table A2.1). These 
estimates represent the total area of the current reservoirs but naturally occurring water bodies were already covering a portion of the landscape prior to the creation of the reservoirs. For example Tecsul (2009) estimated the area actually flooded in BCR 8 at 4,112 ha, nearly 3 times less than the total area covered (Table A2.2). For the rest of the current exercise, we will use the data from Table A2.2 (Tecsul 2009).

\section{Waterfowl breeding densities}

Waterfowl densities vary temporally and spatially throughout the various Canadian ecosystems. Savard and Lamothe (1991) summarise values for northern Quebec and Labrador for scoters (Surf and American Scoters, Melanitta perspicillata and $M$. americana) with a maximum value of 18 pairs $/ 100 \mathrm{~km}^{2}\left(0.18 / \mathrm{km}^{2}\right)$. Transect results yielded an estimate of 0.514 pairs $/ \mathrm{km}^{2}$ for the entire waterfowl community. Lemelin et al. (2004) report densities ranging from 0.89 to 1.33 pairs $/ \mathrm{km}^{2}$ in the forested areas south of $51^{\circ} 015^{\prime} \mathrm{N}$, and Bordage et al. (2002) report a density of 0.86 pairs $/ \mathrm{km}^{2}$ in the area of the Gouin reservoir. None of these estimates have been corrected for detectability biases so they should be considered as minimums. Recent surveys in Labrador yielded waterfowl densities in $25 \mathrm{~m}^{2}$ plots ranging from 0 to 4.14 pairs $/ \mathrm{km}^{2}$ (Table A1.4; Gilliland et al. 2008, 2009). The maximum estimate of 1.33 pairs $/ \mathrm{km}^{2}$ (Lemelin et al. 2004) was used to derive casualties related to flooding of northern reservoirs. The estimate is similar to the mean obtained in Labrador in 2009 (Table A1.4; Gilliland et al. 2009).

\section{Waterfowl species composition}

The abundance and diversity of breeding waterfowl varies throughout the boreal forest depending on location and scale considered (Tables 4 and 5). In the areas affected by northern reservoirs in Quebec, the most numerous waterfowl species average 8.4 eggs/ nest and approximately 8.0 eggs/nest in the area of the reservoir Gouin (Table A1.5). None of the species involved are considered of concern in Canada.

\section{RESULTS}

\section{Electrocutions}

To derive reliable estimates in relation to casualties in Canada due to electrocution, we used data from the literature on the numbers of bird killed/pole/year (Table 2) and applied it to Canada. The total length of distribution lines is estimated at $572370 \mathrm{~km}$. If we assume about 10 poles $/ \mathrm{km}$, we obtain a total of 5723700 poles. Estimates of avian mortality (poles * birds/pole/year) ranged from 160836 (min) to 801962 birds annually. If we exclude the Alberta study, estimates drop to 37490 and 456261 birds per year. Based on the Alberta study (Platt 2005; Table 3), only about $10 \%$ of the birds electrocuted are migratory, whereas the rest are species under provincial jurisdiction. It gives us an estimate from 16084 to 80196 migratory birds electrocuted annually in Canada. 


\title{
Transmission and distribution line construction and maintenance
}

We averaged the breeding bird density of each data source found in Tables 1-3 (3.78; $4.48 ; 3.38)$. This yielded an average of $3.88 \pm 0.56$ pairs/ha $(\mathrm{SD} ; \mathrm{CV}=14.4 \%)$. If we take the maximum from each data base (7.76, DesGranges et al. 2003; 11.9, Kennedy et al. 1999; 6.98, Savard et al. 2000) we obtain an average estimate of $8.88 \pm 2.64$ (SD; CV = $29.7 \%$ ).

Assuming a width of about $100 \mathrm{~m}(0.1 \mathrm{~km})$ we derive an area of $25,175 \mathrm{~km}^{2}$ or 2,517,500 ha of habitats cleared for transmission lines. In the estimates below, we assume that only half of the area was cleared during the breeding season in the following pattern: $10 \%$ in May; $40 \%$ in June; and $50 \%$ in July. We also assume that $20 \%$ of nests are active on any given day in May; $90 \%$ in June; and 30\% in July. Based on these assumptions, we derive two estimates, one based on average values (3.88 pairs/ha) and one based on maximum values (8.88 pairs/ha).

Those yielded respectively casualties of 2,588,494 and 5,924,181 nests related to the construction of transmission lines in Canada. If we change the proportion of nests active in May from $20 \%$ to $50 \%$, in June from $90 \%$ to $90 \%$ and in July from 30 to $50 \%$ we obtain casualties of 3,223,407 and 7,377,282 nests respectively. Also, changing the proportion of clearing done in May, June and July affects estimates only slightly but changing the average breeding bird density has the most impacts (Fig. 1). I should point out that those are cumulative estimates as the construction of transmission lines in Canada was spread over several decades so that the yearly impact was much smaller in any given area.

To derive casualty estimates for maintenance, we assumed that the impact was similar to the one related to the construction phase and occurred only every 5 years or 10 years. Estimates were generated for both scenarios. Assuming a five years recurring management results in estimates that are one fifth of those previously derived $(\mathrm{min}=$ 517,699 nests/year, $\max =1,184,836$ nests/year); if maintenance was only done every 10 years which may be the case in the boreal forest where vegetation growth is slower, casualty estimates are half again $(\min =258,849, \max =592,418)$.

Final casualty estimates based on the authors' unsupported assumptions (educated guess) are: 1) use of average breeding bird density estimates as best representing the variability in habitat types and yearly fluctuations; 2) use of a 5 years recurring maintenance schedule for $60 \%$ of the lines, of 10 years for $30 \%$ and of 0 maintenance for $10 \%$. This scenario yield initial casualties related to construction of 2,588,494 nests and recurring nest mortalities of: $0.60 * 517,699$ nests/year $+0.30 * 258,849=388,274$ nests/year.

\section{Estimated avian mortality in relation to hydro-electric reservoirs}

\author{
Hydro-electric reservoirs in Quebec
}


Approximately $8,342 \mathrm{~km}^{2}$ were inundated by the creation of reservoirs in northern Quebec over the last 40 years (Table A2.2). This yields an estimate of 11095 nests $\left(8,342 \mathrm{~km}^{2} \mathrm{X} 1.33\right.$ pairs $\left./ \mathrm{km}^{2}\right)$ potentially affected if these reservoirs were all filled during the breeding season. However, this is not the case as most large reservoirs take two to three years to fill up covering multiple breeding seasons but affecting different areas. As the breeding season is fairly short in the boreal forest we assumed that waterfowl nests are only vulnerable for a period of two months each year.

Assuming a constant rate of reservoir filling throughout the year over a two year horizon, nests would be vulnerable for four of 24 months (16.6\%). This assumption is questionable as reservoir filling may take more than two years, the area flooded depends on slope and filling would be quicker during spring runoff than at any other time of the year and will depend also on precipitation levels. However, each reservoir situation is different so that it is difficult to determine adequate assumptions. If we apply this percentage $(16.6 \%)$ to the $8,342 \mathrm{~km}^{2}$ flooded area, $1,385 \mathrm{~km}^{2}$ would have been affected during the breeding season. This translates into 1,842 waterfowl nests that could have been affected.

Assuming an average clutch size of 8.4 eggs (Table A2.2; weighted average), it represents 15,473 eggs lost in relation to the filling of the reservoirs. This would have been a one-time impact.

\section{Other reservoirs in Quebec}

Lehoux et al. (1991) conducted a study of the impact of fluctuating water levels in the Montreal/lac Saint-Pierre sector of the St. Lawrence River. These fluctuations were not directly related to hydro-electricity but were mostly for navigation considerations. However, because of the high productivity of the impacted areas, it affects productive waterfowl habitats and results in casualties. Impacts varied yearly with the level of water fluctuation and its timing (Fig. 2). Waterfowl breeding densities in the freshwater portion affected by water level controls were evaluated at 23 nests $/ \mathrm{km}^{2}(0.23 \mathrm{nest} / \mathrm{ha})$, densities very high compared to northern forested habitats. As expected, the highest impacts occur with the highest water levels and the number of nests potentially impacted varies with the timing in regard to nesting chronology (Fig. 2). It should be noted that increase in water levels before or after the breeding season will not result in casualties. Unlike reservoirs for which the major impact was at the time of filling, in the case of the St. Lawrence, it occurs at various levels every year and with various timing, making it difficult to generate credible estimates.

\section{British Columbia}

A detailed study of avian nest mortality in relation to water level fluctuations in reservoirs was made for the Kinbasket and Arrow Lakes reservoirs in the Columbia valley of British Columbia (BC Hydro unpublished data). Most nest failures were due to predation $(78.3 \%, \mathrm{n}=258$ nests, in the Arrow Lakes Reservoir and 77\%, $\mathrm{n}=352$ nests, in 
the Kinbasket Reservoir). The failure of seven nests (5.8\%) was directly caused by reservoir operations, all in the Arrow Lakes Reservoir. No nest losses could be attributed to reservoir operations in the Kinbasket Reservoir. Clearly, in this study at least, avian mortality was low. However a study of the drawdown zones of the Columbia River reservoir network documented several nest flooded by rising water levels but concluded that impacts were negligible from a population perspective but could be significant locally when locally rare species are affected (BC Hydro unpublished data).

\section{Other potential losses of non-waterfowl species due to reservoir flooding}

Activities related to the initial creation of reservoirs may cause some avian mortality when the habitat is modified prior to flooding. However, the area affected cannot be adequately measured as habitat modification does not occur in all reservoirs (in some, trees are cut prior to flooding, in others not). Also, there is no easily available information as to the period when the clearing was done. Assuming that $75 \%$ of the reservoir area in northern Quebec was cleared $\left(6,257 \mathrm{~km}^{2}\right.$ or $\left.625,700 \mathrm{ha}\right)$ and that half of the clearing was done during the breeding season $\left(3,129 \mathrm{~km}^{2}\right.$ or $\left.312,850 \mathrm{ha}\right)$ we obtain, assuming that all the area cleared was forested and sustained a density of breeding birds of 37.8 pairs/10ha (3.78/ha; Table A1.5), an estimate of 1182573 nests affected. This is very likely a maximum estimate as breeding bird densities in these areas are likely lower and that a mixture of habitats were affected. Hobson (2011) calculated that the proportion of forest harvesting occurring during the breeding season in Canada ranged from a low of $12 \%$ and a high $26 \%$. Application of these percentages here yield casualties of 280,063 nests $(75,084$ ha $* 3.73$ nest/ha) and 606,804 nests $(162,682 * 3.73$ nest/ha $)$, respectively.

In the worst case scenario, water levels would rise quickly to the 7.98 meter at Sorel in early to mid-June. Such a scenario could impact 600 nests which, with an average clutch size of 8 eggs would represent 4800 eggs. Clearly, such a scenario would significantly impact the productivity of the local waterfowl population estimated at about 900 nests.

\section{Impacts at the population level}

Indeed the worst case scenario in Northern Quebec, the province with the greatest number of reservoirs in terms of area, resulted in less than 1842 nests affected total and, this distributed across several species. The impact of such losses at the population level will have to consider natural mortality at both the nest and brood stages. As mortality factors vary greatly from year to year, the impact on the local population will also vary accordingly. Assuming a survival of $50 \%$ of nests and a $50 \%$ survival of broods reduce the impact to a loss of 461 waterfowl nests and of (using Hobson 2011 estimate of $26 \%$ harvest during the breeding season) 151,701 non waterfowl nests . Thus, once natural mortality is considered, a total of 152,162 nests were lost due directly to reservoir flooding.

\section{DISCUSSION}


Summarizing calculations from multiple sources analyzed above, we obtain the combined estimates of casualties caused by power lines ranging from 160,836 to 801,962 adult birds and 388,274 nests in Canada, annually. Due to lack of data, we cannot provide a reliable estimate of casualties caused by hydro-power reservoirs for Canada, but Quebec, the province with the greatest number of reservoirs in terms of area gives us 152,162 nests affected.

\section{Electrocution}

Electrocutions are mostly associated with distribution lines and are relatively uncommon on transmission lines, which is the reverse for collision casualties. Clearly better data are needed, especially for poles in the boreal forest which may not cause excessive mortality because of habitat structure, species composition and behaviour of raptors there. We also need more accurate data for open areas, i.e. prairies with greater raptor densities, where poles provide an attractive structure for hunting and resting birds. The abundance and types of poles in Canada need to be quantified better, as electrocution risks are directly related to pole types. Their spatial occurrence has to be evaluated as risks vary greatly for a given type of pole depending on the surrounding habitat.

Due to relatively small amount of migratory birds reported electrocuted (most are raptors and owls), mortality of migratory birds due to electrocution cannot be properly evaluated. Thus impacts at the population level are hard to assess. Impacts are likely to be greater for the long-lived species, such as raptors, owls, herons, and cranes than on smaller, short-lived, but highly productive birds. In some cases, local populations of some owls and raptors could be significantly affected (Sergio et al. 2004). Newly developed strategies to identify problematic poles have been focused on modeling to identify problematic pole types (Manosa 2001, Tinto et al. 2010) but this approach has still to be applied in Canada. Electrocution of birds has not been recognised as a national issue in Canada, in part because of the focus of the literature on large raptors and lack of studies.

\section{Construction and maintenance}

To derive accurate estimates of avian mortality related to the construction of transmission lines, we need an estimate of the length of diverse habitats traversed by these lines throughout Canada as well as estimates of breeding bird densities within these habitats. It also requires an estimate of the period of the year when vegetation was cleared, which is not currently available.

On transmission system lines, the wires are not insulated by a sheath and air acts as the insulator. When vegetation comes close to the conductors (wires), there is a risk of an electrical arc forming which may cause a power outage, start a fire and even electrocute people in the vicinity. To avoid this occurrence, vegetation under transmission lines has to be kept relatively short and thus need to be managed on a regular basis. 
For Hydro-Québec, the vegetation management period begins after snow melting in spring and continues into the fall. Vegetation control under transmission lines is done about every five years, depending on the climate zone and the method used. The farther north a line is located, the less frequent the clearing operations, since vegetation at higher latitudes tends to grow at a slower rate.

Hydro-Québec uses three methods for clearing rights-of-way, either alone or in combination: a) selective cutting (using chainsaws, brush cutters and mowers); b) selective application of herbicides (pesticides that kill certain plants or inhibit their growth, while allowing other plants to develop); c) land-use development (bicycle paths, crop cultivation, gardens, etc.). The last one creates habitats not suitable for breeding. In general, Hydro-Québec uses only mechanical cutting to control vegetation in rights-ofway in $70 \%$ of all cases. The other work consists of a combination of mechanical cutting and selective application of herbicides. As the proportion of lines managed by each method is unknown and given that the use of herbicide is often preceded by vegetation clearing, it will be assumed here that all methods result in similar impact. The maintenance of distribution lines may result in some nest mortalty but it is done differently and involves mostly branch trimming rather than whole tree removal.

\section{Reservoirs}

Losses associated with creation of hydro-electric reservoirs at the population level are not significant locally, globally, and at the species level, for most if not all species of waterfowl, considering the populations of several millions. However, we have to take into account that losses are calculated for Quebec only and do not include other provinces. More factual data on each reservoir in each province are needed to obtain refined estimates.

This exercise, although highly speculative, suggests that avian mortality related to reservoir flooding is negligible at provincial scale, and it is unlikely to cause significant impact for any species. One cautionary note: reservoirs within the breeding range of the Piping Plover (Charadrius melodus), an endangered species could be used for nesting by the species, rendering nests susceptible to flooding and could affect the local population if such losses were recurrent every year.

There is no impact related to vegetation removal if it is done outside of the breeding season. Often, prior to reservoir flooding, trees are cut to reduce boating hazards and limit the vegetation decomposition, which affects water chemistry. However, within the breeding season the impact will be similar to that of forest harvesting (in forested areas) and mostly limited to the destruction of eggs and/or nestlings, whereas adults remain unaffected. Therefore, nest mortality is proportional to the number of active nests in the harvested area.

Similarly, no impact related to flooding occurs outside the breeding season. If done during the breeding season, it will affect mostly ground nesting species in the flooded 
area. In some cases, especially when shrubs are abundant, it will harm shrub nesting species and could even take species breeding in small trees.

Most artificial reservoirs have fluctuating water levels in relation to precipitation and water usage. These levels often do not coincide with natural water fluctuations and could flood nests built in the affected zone (Nilsson and Dynesius 1994, Lehoux et al. 2003). In most large reservoirs $\left(\mathrm{km}^{2}\right)$, especially those with unnatural and important fluctuations (a few meters), little vegetation is established in the affected zone, and nesting is minimal. However, in the north of Canada, and also possibly in the prairies, this bare zone attracts some species of ground nesting shorebirds. In those cases, primarily eggs are impacted as the nidifugous young can avoid flooding (they may perish indirectly due to lack of habitat and greater susceptibility to predation). However, the impact is limited to the breeding season, mostly eggs and nestlings, and it is local. In the special case of the Great LakesSt. Lawrence system where water levels are controlled for navigation, unnatural water fluctuations occur often and have potential of flooding waterfowl, terns and other ground nesting waterfowl nests (Lehoux et al. 1991).

Unfortunately, reliable estimates for the area flooded by reservoirs in most of the other provinces could not be obtained. However, it is much smaller than the area in Quebec, and in general most reservoirs in other provinces are smaller. There might be localised losses due to fluctuations in water levels each year but this is very likely insignificant as for most reservoirs, the marnage zone is often devoid of vegetation and not very attractive for ground nesting birds. Besides, to cause an impact, the water rise would have to coincide with the nesting period. Furthermore, the area affected by flooding due to yearly water fluctuations is much smaller than the area initially flooded. However, if flooding occurs each year at the time birds have already initiated their breeding activities, it would result in recurring yearly losses. These conditions vary from reservoir to reservoir, and they require local data to estimate losses.

\section{CONCLUSIONS}

Given IT numbers for electrocutions unlikely result in significant population effect on individual species with possibly some exception for some endangered species such as Whooping Cranes (Grus americana). Nest mortality related to the construction and maintenance of transmission and distribution power lines was grossly estimated but seems relatively small, and would not cause any significant impact at the population level. Nest mortality caused by hydro-power reservoirs is considered negligible, however, it is highly speculative, in great part because of the coarseness of the data available on reservoirs size, the way they were filled, and the yearly water fluctuations and timing. Data on breeding bird densities could also be refined. Finally, detailed studies similar to those in British Columbia (BC Hydro unpublished data) or to Lehoux et al. (2003) in the St. Lawrence are greatly needed to derive more realistic and credible estimates for each reservoir.

\section{ACKNOWLEGEMENTS}


We would like to thank Geoff Holroyd for his pertinent comments and suggestions, and Dr. Robert Elner for his insightful comments.

\section{LITERATURE CITED}

Avian Power Line Interaction Committee (APLIC). 2006. Suggested practices for avian protection on power lines - the state of the art in 2006. Edison Electric Institute, Washington, D.C., USA.

Anderson, A. H. 1933. Electrocution of Purple Martins. Condor 35:204.

Baxter, R. M., and P. Claude. 1980. Environmental effects of dams and impoundments in Canada: experience and prospects. Canadian Bulletin of Fishery and Aquatic Sciences 205:1-34.

Benson, P. C. 1981. Large raptor electrocution and power pole utilisation: a study in six western states. Dissertation. Brigham Young University, Provo, Utah, USA.

Bevanger, K. 1995. Estimates and population consequences of tetranoid mortality caused by collisions with high tension power lines in Norway. Journal of Applied Ecology 32:745-753.

Bevanger, K. 1998. Biological and conservation aspects of bird mortality caused by electric power lines: a review. Biological Conservation 86:67-76.

Bevanger, K. 1999. Estimating bird mortality caused by collision and electrocution with power lines: a review of methodology. Pages 29-56 in M. Ferrer and G. F. Janss, editors. Birds and power lines: collision, electrocution, and breeding. Quercus, Madrid, Spain.

Boeker, E. L., and P. R. Nikerson. 1975. Raptor electrocutions. Wildlife Society Bulletin 3:79-81.

Books, G. G. 1985. Avian interactions with Mid-Columbia River water level fluctuations. Northwest Science 59: 304-312.

Bordage, D., M. Grenier, N. Plante, and C. Lepage. 2002. Répartition potentielle de la sauvagine dans la region du reservoir Gouin, Québec. Technical Report Series No. 374, Canadian Wildlife Service, Sainte-Foy, Canada.

Bramble, R. H., R. H. Yahner, and W. R. Byrnes. 1994. Nesting of breeding birds on an electric utility line right-of-way. Journal of Arboriculture 20:124-129.

Cartron, J.-L. E., R. E. Harness, R. C. Rogers, and P. Manzano-Fisher. 2005. Impact of concrete power poles on raptors and ravens in northwestern Chihuahua, Mexico. Pages 357-369 in J.-L. Cartron, G. Ceballos and R. S. Felger, editors. Biodiversity,ecosystems, 
and conservation in northern Mexico. Oxford University Press, New York, New York, USA.

Dawson, J. W., and R. W. Mannan. 1995. Electrocution as a mortality factor in an urban population of Harris' Hawks. Journal of Raptor Research 29:55.

Dedon, M. F., and E. W. Colson. 1988. Investigation of bird-caused outages in the Pacific gas and electric company service area. Pages 34-45 in W. R. Byrnes and H. A. Holt, editors. Proceedings of the fourth Symposium on environmental concerns in Rights-ofway management. Purdue University, Department of Forestry and Natural Resources, West Lafayette, Indiana, USA.

DesGranges, J.-L., Y. Grégoire, P. Belleau, P. Agin, and L. Coté. 2003. Identification d'aires prioritaires de conservation par l'analyse de la diversité aviaire: le cas des forêts du Bas St-Laurent. Technical Report Series No. 386, Canadian Wildlife Service, Quebec, Canada.

Dexter, R. W. 1953. Electrocution of a Baltimore Oriole. Journal of Field Ornithology 24:109.

Ferrer, M., M. De La Riva, and J. Castroviejo. 1991. Electrocution of raptors on power lines in Southern Spain. Journal of Field Ornithology 62:54-69.

Gilliland, S. G., C. Lepage, J.-P. L. Savard, D. Bordage, and G. J. Robertson. 2009. An assessment of distribution and abundance of Surf and Black Scoters breeding within the eastern section of Labrador low level Flight Training Area 732. Report submitted to the Institute for Environmental Monitoring and Research, Happy Valley-Goose Bay, Labrador. (www.iemr.org/pdfs/R_Waterfowl/Scoter09_english.pdf·PDF file)

Gilliland, S. G., C. Lepage, J.-P. L. Savard, D. Bordage and G. J. Robertson. 2008. An assessment of distribution and abundance of Surf and Black Scoters breeding within the eastern section of Labrador low level Flight Training Area 732. Report submitted to the Institute for Environmental Monitoring and Research, Happy Valley-Goose Bay, Labrador. (www.iemr.org/pdfs/R_Waterfowl/Scoter08_english.pdf·PDF file)

Harness, R. E., and Wilson, K. R. 2001. Electric-utility structures associated with raptor electrocutions in rural areas. Wildlife Society Bulletin 29:612-623.

Hobson, K. A. 2011. An estimate of incidental take of nests of forest birds in Canada due to forestry operations. Unpublished Report, Science and Technology, Environment Canada, (Branch, City?),Canada..

Janss, G. F. E. 2000. Avian mortality from power lines: a morphologic approach of a species-specific mortality. Biological Conservation 95:353-359. 
Janss, G. F. E., and M. Ferrer. 1999a. Avian electrocution on power poles: European experiences. Pages 145-159 in M. Ferrer and G. F. E. Janss, editors. Birds and power lines: collision, electrocution, and breeding. Quercus, Madrid, Spain.

Janss, G. F. E., and M. Ferrer. 1999b. Mitigation of raptor electrocution on steel power poles. Wildlife Society Bulletin 27:263-273.

Janss, G. F. E., and M. Ferrer. 2001. Avian electrocution mortality in relation to pole design and adjacent habitat in Spain. Bird Conservation International 11:3-12.

Kennedy, J. A., P. Dilworth-Christie, and A. J. Erskine 1999. The Canadian breeding bird (mapping) census database. Technical Report Series No. 342, Canadian Wildlife Service, Ottawa, Canada.

Kochert, M. N., and R. R. Orlendoff. 1999. Creating raptor benefits from power line problems. Journal of Raptor Research 33:39-42.

Lammers, W. M., and M. W. Collopy. 2007. Effectiveness of avian predator perch deterrents on electric transmission lines. Journal of Wildlife Management 71:2752-2758.

Ledger, J. A. 1984. Engineering solutions to the problem of vulture electrocutions on electricity towers. Certified Engineer 57:92-95.

Lehman, R. N. 2001. Raptor electrocution on power lines: current issues and outlook. Wildlife Society Bulletin 29:804-813.

Lehman, R. N., P. L. Kennedy and J. A. Savidge. 2007. The state of the art in raptor electrocution research: a global view. Biological Conservation 136:159-174.

Lehman, R. N., J. A. Savidge, P. L. Kennedy, and R. E. Harness. 2010. Raptor electrocution rates for a utility in the intermountain western United States. Journal of Wildlife Management 74:459-470.

Lehoux, D., D. Dauphin, O. Champoux, J. Morin and G. Létourneau. 2003. Impacts des fluctuations des niveaux d'eau sur les canards barboteurs en reproduction dans le tronçon lac Saint-Louis/lac Saint-Pierre. Environment Canada, Canadian Wildlife Service, Sainte-Foy, Canada.

Lemelin, L.-V., D. Bordage, M. Darveau and C. Lepage. 2004. Répartition de la sauvagine et d'autres oiseaux utilisant les milieux aquatiques en période de nidification dans le Québec forestier. Technical Report Series No. 422. Environment Canada, Canadian Wildlife Service, Sainte-Foy, Canada.

Manosa, S. 2001. Strategies to identify dangerous electricity pylons for birds. Biological Conservation 10:1997-2012. 
Manville, A. M. II. 2005. Bird strikes and electrocution at power lines, communication towers, and wind turbines: state of the art and state of the science-next step toward mitigation. U.S. Forest Service General Technical Report PSW-GTR-191, Washington D.C., USA.

Manzano-Fischer, P. 2006. Power-line electrocution of birds (summary). Pages 69-70 in X. Basurto, and D. Hadley, editors. Grasslands ecosystems, endangered species and sustainable ranching in the Mexico-U.S. borderlands: conference proceedings. RMRS-P40. Fort Collins, CO., U.S. Department of Agriculture, Forest Service, Rocky Mountain Research Station.

Miller, D., E. L. Boeker, R. S. Thorsell, and R. R. Orlendoff. 1975. Suggested practices for raptor protection on power lines. Electric Institute, Washington D. C., and Raptor Research Foundation, Provo, Utah, USA.

Nilsson, C., and M. Dynesius. 1994. Ecological effects of river regulation on mammals and birds: a review. Regulated Rivers: Research and Management 9:45-53.

Orlendorff, R. R., A. D. Miller, and R. N. Lehman. 1981. Suggested practices for raptor protection on power lines: the state of the art in 1981. Raptor research report No. 4. Raptor Research Foundation, Provo, Utah, USA.

O'Neil, T. 1988. An analysis of bird electrocutions in Montana. Journal of Raptor Research 22:27-28.

Platt, C. M. 2005. Patterns of raptor electrocution mortality on distribution power lines in southeast Alberta. Dissertation, University of Alberta, Edmonton, Canada.

Roig-Soles, J., and V. Navazo-Lopez. 1997. A five-year Spanish research project on bird electrocution and collision with electric lines. Pages 317-325 in J. R. Williams, J. W. Goodrich-Mahoney, J. R. Wisniewski and J. Wisniewski, editors. The sixth international symposium on environmental concerns in rights-of-way management. New Orleans, Louisiana, USA.

Rubolini, D., E. Bassi, G. Bogliana, P. Galeotti, and R. Garavaglia. 2001. Eagle Owl Bubo bubo and power line interactions in the Italian Alps. Bird Conservation International 11:319-324.

Rubolini, D., M. Gustin, G. Bogliana, and R. Garavaglia. 2005. Birds and power lines in Italy: an assessment. Bird Consevation International 15:131-145.

Savard, J.-P. L., and P. Lamothe. 1991. Distribution, abundance, and aspects of breeding ecology of Black scoters, Melanitta nigra, and Surf Scoters, M. perspicillata, in Northern Quebec. Canadian Field-Naturalist 105:488-496. 
Savard, J.-P. L., D. A. Seip, and L. Waterhouse. 2000. Avian diversity in relation to logging in the coastal rainforests of British Columbia. Technical Report Series No. 349, Canadian Wildlife Service, Pacific and Yukon Region, Delta, Canada.

Sergio, F., L. Marchesi, P. Pedrini, M. Ferrer, and V. Penteriani. 2004. Electrocution alters the distribution and density of a top predator, the Eagle Owl Bubo bubo. Journal of Applied Ecology 41:836-845.

Slater, S. J., and J. P. Smith. 2010. Effectiveness of raptor perch deterrents on an electrical transmission line in Southern Wyoming. Journal of Wildlife Management 74:1080-1088.

Smallwood, K. S. 2007. Estimating wind turbine-caused bird mortality. Journal of Wildlife Management 71:2781-2791.

Tecsult Inc. 2009. Portrait détaillé de l'occupation humaine du territoire et des activités reliées aux divers secteurs de l'industrie des six régions de conservation des oiseaux du Québec. Québec, Canada.

Tinto, A., J. Real, and S. Manosa. 2010. Predicting and correcting electrocution of birds in Mediterranean areas. Journal of Wildlife Management 74:1852-1862.

Wolf, K. 1955. Some effects of fluctuating and falling water levels on waterfowl production. Journal of Wildlife Management 19:13-23.

Yahner, R. H., B. D. Ross, R. T. Yahner, R. J. Hutnik, and S. A. Liscinski. 2004. Long term effects of rights-of-way maintenance via the wire-border zone method on bird nesting ecology. Journal of Arboriculture 30:288-294. 
Table 1. Number of birds received by the 'Union Québécoise de réhabilitation des oiseaux de proie' between 1995 and 2009.

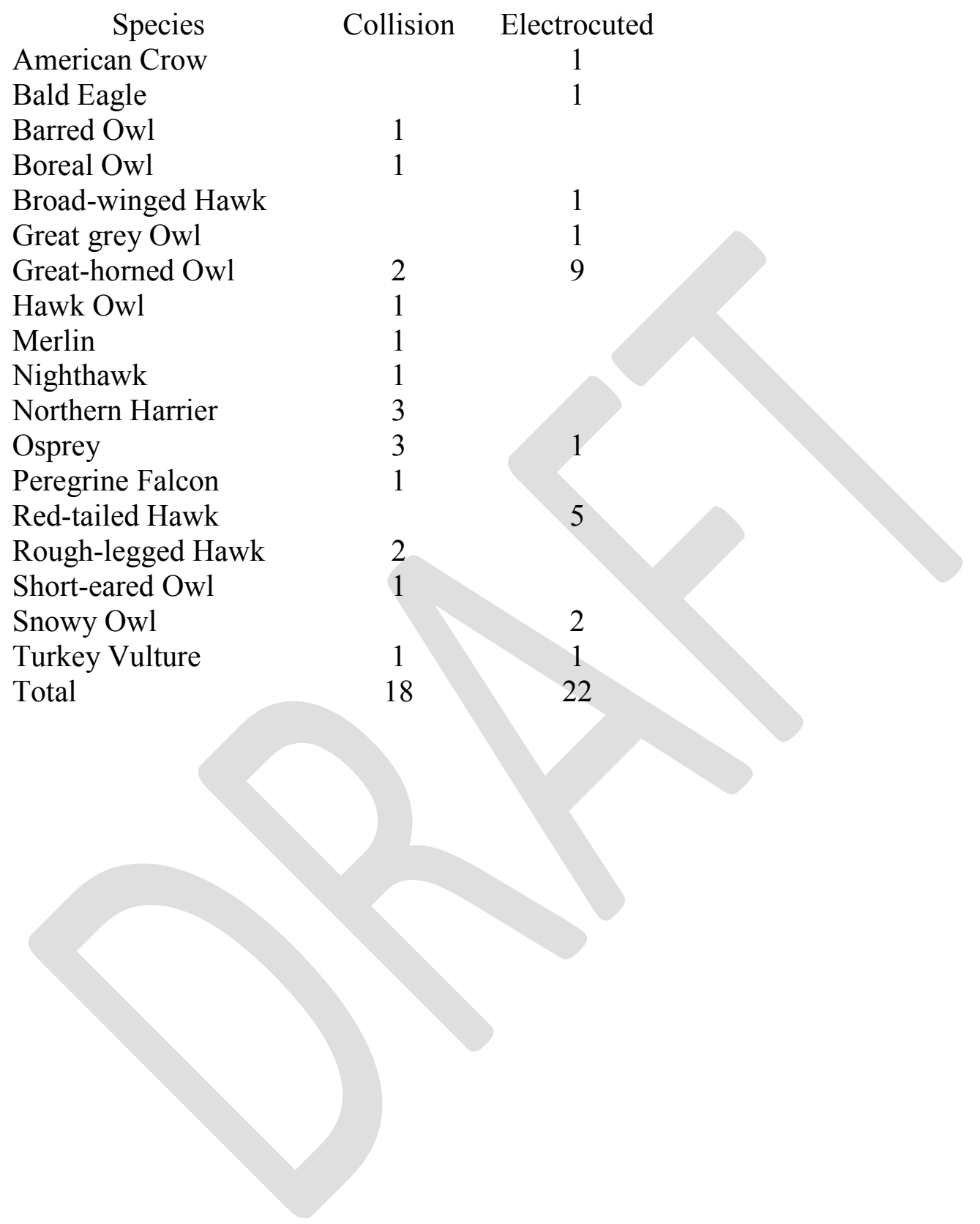


Table 2. Estimate of bird electrocution rates from the literature.

\begin{tabular}{|c|c|c|c|c|c|c|c|}
\hline Location & $\begin{array}{c}\text { Bird/ } \\
\text { pole }\end{array}$ & $\begin{array}{l}\text { Length } \\
\text { of } \\
\text { study }\end{array}$ & $\begin{array}{l}\text { Period } \\
\text { covered }\end{array}$ & Year & $\begin{array}{c}\text { Minimum } \\
\text { estimate } \\
\text { bird/pole/ } \\
\text { year }\end{array}$ & $\begin{array}{c}\text { Maximum } \\
\text { estimate } \\
\text { bird/pole/ } \\
\text { year }\end{array}$ & Source \\
\hline Spain & 0.13 & 334 & Annual & $1982-1983$ & 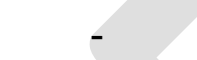 & 0.1377 & Ferrer et al. 1991 \\
\hline Utah & - & - & Annual & $2001-2003$ & 0.0112 & - & Lehman et al. 2010 \\
\hline Utah & - & - & Annual & $2001-2003$ & 0.0036 & - & Lehman et al. 2010 \\
\hline Utah & - & - & Annual & $2001-2003$ & 0.0045 & 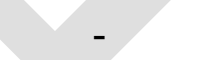 & Lehman et al. 2010 \\
\hline Spain & 0.03 & 365 & Spring\&Fall & 1991-1995 & & 0.0311 & Janss et Ferrer 1999a \\
\hline Spain & 0.23 & 549 & Annual & 1993-1994 & & 0.1541 & Janss et Ferrer 1999a \\
\hline USA & $?$ & $?$ & $?$ & $?$ & 0.0069 & 0.0176 & Lehman et al. 2010 \\
\hline Alberta $^{\dagger}$ & 0.21 & 43 & Summer & 2003 & 0.1143 & 0.5629 & Platt 2005 \\
\hline Spain & 0.01 & 1492 & Annual & 1995- 1999 & & 0.0019 & Manosa 2001 \\
\hline Spain & 0.11 & 1492 & Annual & 1995- 1999 & 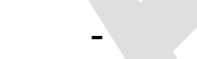 & 0.0259 & Manosa 2001 \\
\hline Spain & 0.78 & 1492 & Annual & 1995- 1999 & . & 0.1897 & Manosa 2001 \\
\hline Average & & & & & 0.0281 & 0.1401 & \\
\hline Average $2^{*}$ & & & & & 0.0066 & 0.0797 & \\
\hline IT & & & & & 160836 & 801962 & This study \\
\hline $\mathrm{IT}^{*}$ & & & & & 37490 & 456261 & This study \\
\hline
\end{tabular}

${ }^{\dagger}$ The study was done over a 6 weeks period in June and July. To derive annual estimates we extrapolated to 6 months or 20 weeks (May to October included). Rates: 64 birds for 379 poles during 6 weeks $=0.1689$ birds/pole/ 6 weeks; this yield an estimate of 0.5629 birds/pole/20 weeks. We assumed no death from November to late April. Only 13 birds were confirmed as electrocuted; this yields a minimum estimate of $0.1143 \mathrm{birds} /$ pole/ 20 weeks.

Excluding the Alberta study. 
Table 3. Number of individuals recovered during a 6 weeks period (June and July) in Alberta during two searches of 379 poles (Only 13 of the birds recovered could be confirmed as being electrocuted). Scavenging efficiency was estimated at $62 \%$ over this period.

\begin{tabular}{lcc}
\hline \multicolumn{1}{c}{ Species } & $\begin{array}{c}\text { Numbers } \\
\text { recovered }\end{array}$ & $\%$ \\
\hline Raptors & 11 & 12 \\
Great Horned Owl & 6 & 6 \\
Red-tailed Hawk & 4 & 4 \\
American Kestrel & & 0 \\
Golden Eagle & 3 & 3 \\
Raptor sp. & & \\
Non-raptors & 5 & 5 \\
Black-billed Magpie & 2 & 2 \\
Sharp-tailed Grouse & 2 & 2 \\
Duck & 2 & 2 \\
Blackbird sp. & 3 & 3 \\
Passerine sp. & 12 & 13 \\
Corvid & 0 & 0 \\
Gull & 0 & 0 \\
Northern Flicker & 14 & 15 \\
Unknown & & \\
Total migratory birds & 7 & 7 \\
Total raptors & 24 & 26 \\
Total other non-migratory & & \\
birds & 19 & 20 \\
Unknown & 14 & 15 \\
Grand total & $\mathbf{6 4}$ & \\
\hline
\end{tabular}


Table 4. Estimates of the number of nests lost in relation to the construction and maintenance of transmission lines.

\begin{tabular}{|c|c|c|c|c|c|c|c|c|}
\hline \multicolumn{5}{|c|}{ Construction } & \multicolumn{2}{|c|}{$\begin{array}{l}100 \%{ }^{\dagger} \text { during breeding } \\
\text { season }\end{array}$} & \multicolumn{2}{|c|}{$\begin{array}{l}50 \%{ }^{\dagger} \text { during breeding } \\
\text { season }\end{array}$} \\
\hline May & 0.20 & 0.776 & 1.776 & 0.10 & 195,358 & 447,108 & 97,679 & 223,554 \\
\hline June & 0.90 & 3.492 & 7.992 & 0.40 & $3,516,444$ & $8,047,944$ & $1,758,222$ & $4,023,972$ \\
\hline July & 0.30 & 1.164 & 2.664 & 0.50 & $1,465,185$ & $3,353,310$ & 732,593 & $1,676,655$ \\
\hline Total & & & & & $5,176,987$ & $11,848,362$ & $2,588,494$ & $5,924,181$ \\
\hline \multicolumn{9}{|c|}{ Maintenance at 5 years interval } \\
\hline May & 0.20 & 0.776 & 1.776 & 0.10 & 39,072 & 89421.6 & 19,536 & 44,711 \\
\hline June & 0.90 & 3.492 & 7.992 & 0.40 & 703,289 & 1609589 & 351,644 & 804,794 \\
\hline July & 0.30 & 1.164 & 2.664 & 0.50 & 293,037 & 670662 & 146,519 & 335,331 \\
\hline Total & & & & & $1,035,397$ & 2369672 & 517,699 & $1,184,836$ \\
\hline \multicolumn{9}{|c|}{ Maintenance at 10 years interval } \\
\hline May & 0.20 & 0.776 & 1.776 & 0.10 & 19,536 & 44,711 & 9,768 & 22,355 \\
\hline June & 0.90 & 3.492 & 7.992 & 0.40 & 351,644 & 804,794 & 175,822 & 402,397 \\
\hline July & 0.30 & 1.164 & 2.664 & 0.50 & 146,519 & 335,331 & 73,259 & 167,666 \\
\hline Total & & & & & 517,699 & $1,184,836$ & 258,849 & 592,418 \\
\hline
\end{tabular}

${ }^{\dagger}$ Assuming all maintenance activities (100\%) are done in May, June and July or that only 50\% are.

‡ Number of nests affected 
Table 5. Relative abundance of ground nesting waterfowl in northern Quebec in the area of important Hydro-electric reservoirs (Savard and Lamothe 1991).

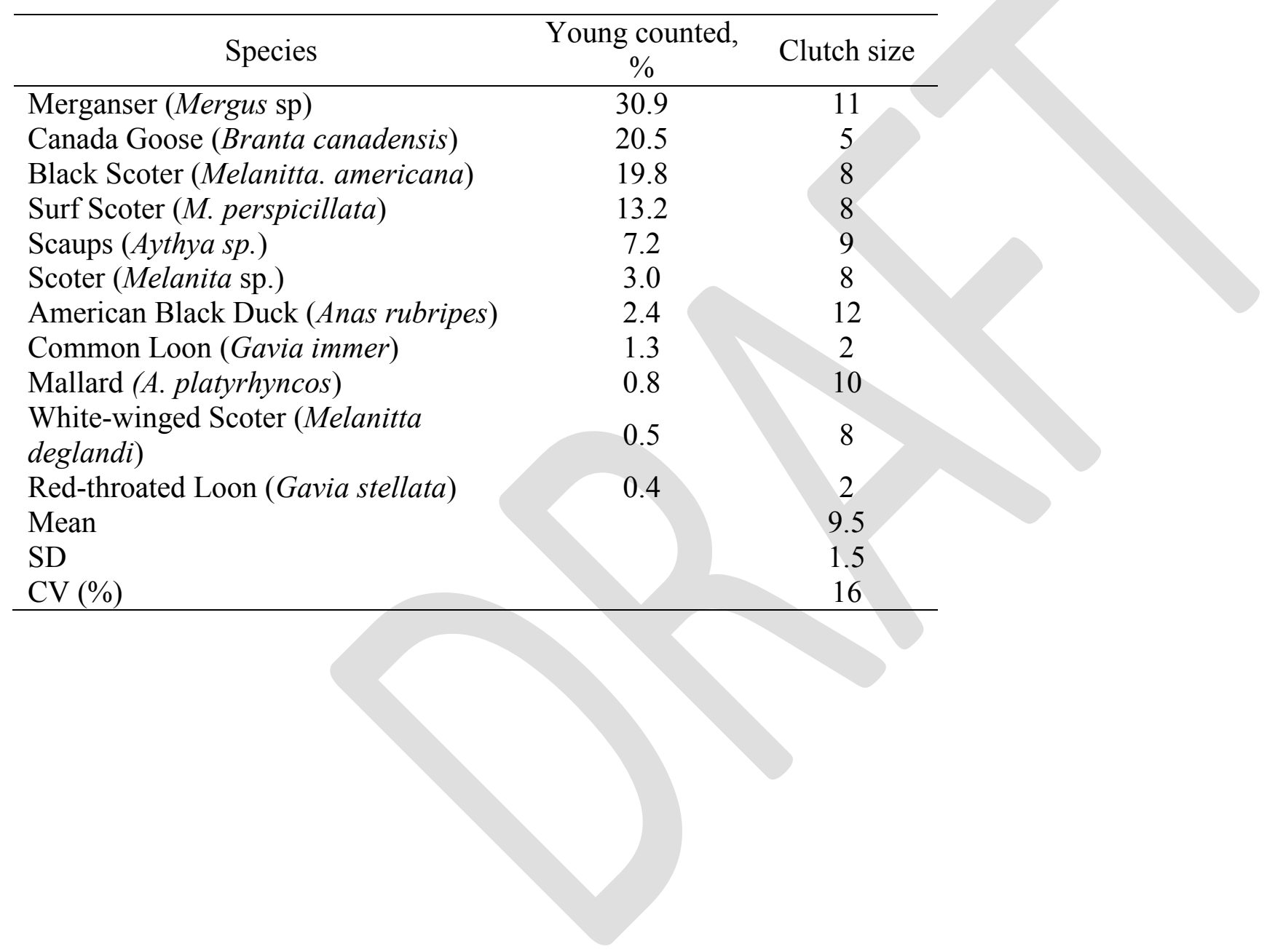


Table 6. Relative abundance of waterfowl (\% of pairs observed) in northern Quebec near the Hydro-electric reservoir Gouin (Bordage et al. 2002).

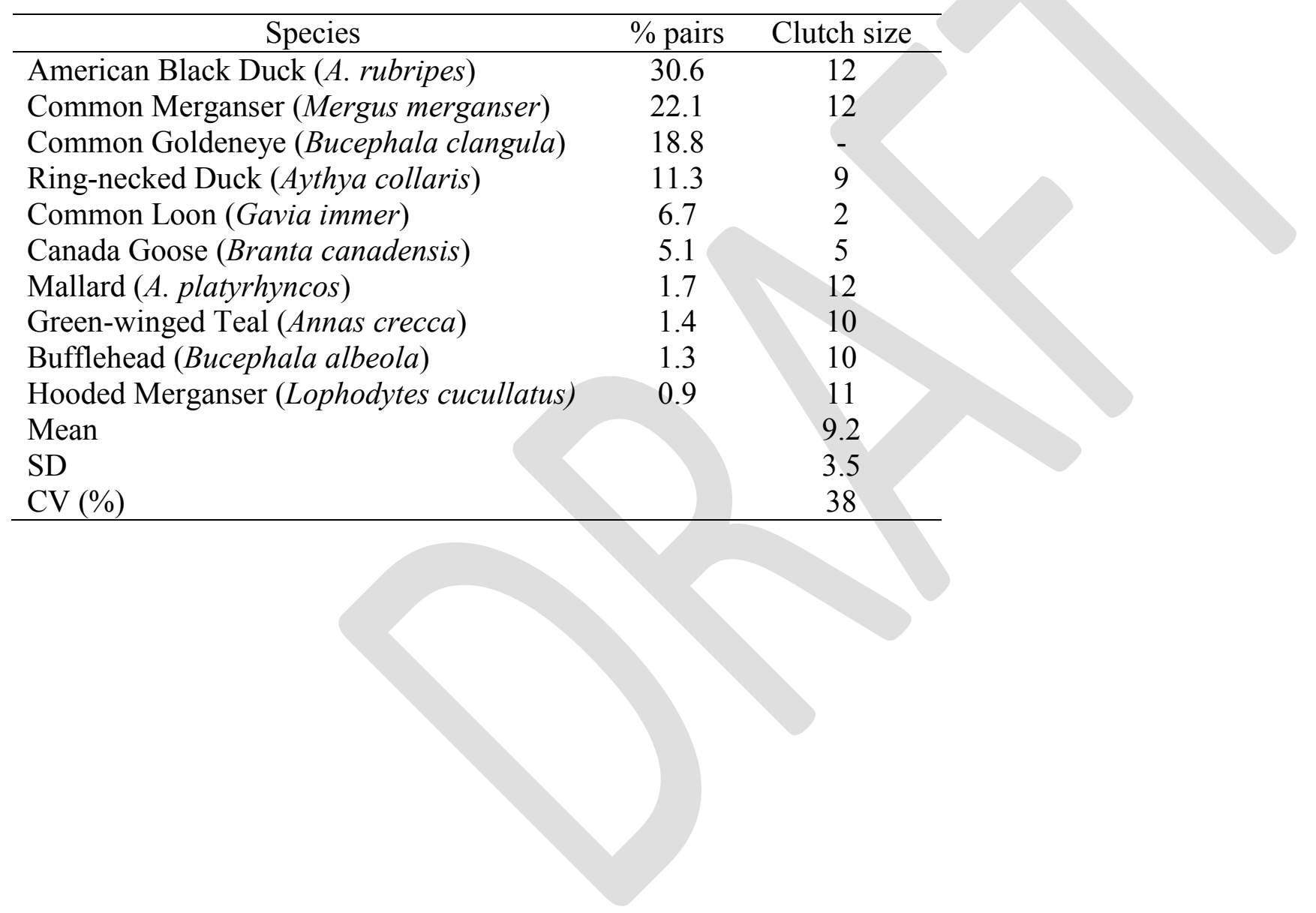


APPENDIX 1. Breeding bird density estimates from the literature.

Table 1. Breeding birds densities (pairs/ha) in major forest types in Quebec (DesGranges et al. 2003).

\begin{tabular}{lccccc}
\hline \hline Forest type & Mean & SD & SE & n & CV \\
\hline Maple & 4.64 & 1.14 & 0.57 & 4 & 25 \\
Aspen & 3.90 & 1.51 & 0.87 & 3 & 39 \\
Balsam-fir & 4.09 & 0.92 & 0.41 & 5 & 22 \\
Birch & 3.50 & 0.83 & 0.48 & 3 & 24 \\
Sruce & 2.73 & 0.90 & 0.37 & 6 & 33 \\
Poplar & 4.06 & - & - & 1 & 0 \\
Larch & 3.76 & 0.65 & 0.46 & 2 & 17 \\
Pine & 3.53 & 0.21 & 0.12 & 3 & 6 \\
Average & $\mathbf{3 . 7 8}$ & $\mathbf{0 . 5 6}$ & $\mathbf{0 . 2 0}$ & $\mathbf{8}$ & $\mathbf{1 5}$ \\
\hline
\end{tabular}

Table 2. Breeding birds densities (Pairs/ha) in major forest types in Quebec (Kennedy et al. 1999).

\begin{tabular}{lcc}
\hline \multicolumn{1}{c}{ Primary habitats } & Low & High \\
\hline Broad-leafed forest/woodland & 1.70 & 5.95 \\
Conifer and mixed & 0.17 & 4.60 \\
forest/woodland & 0.73 & 5.09 \\
Wetlands & 0.22 & 3.51 \\
Open land & 2.00 & 3.25 \\
Urban area & $\mathbf{0 . 9 6}$ & $\mathbf{4 . 4 8}$ \\
Average & $\mathbf{( 0 . 8 5 )}$ & $\mathbf{( 1 . 1 2 )}$ \\
(SD) & $\mathbf{( 0 . 3 5 )}$ & $\mathbf{( 0 . 4 6 )}$ \\
(SE) & & \\
\hline
\end{tabular}


Table 3. Breeding bird densities (Pairs/ha) in coastal forests of British Columbia (Savard et al. 2000).

\begin{tabular}{llcccc}
\hline \hline Location & \multicolumn{1}{c}{ Forest type } & Mean & SE & n & Range \\
\hline Queen Charlotte Islands & Old growth & 4.64 & 0.67 & 3 & $3.32-5.17$ \\
& 40-80 years old & 3.17 & 0.06 & 3 & $3.12-3.29$ \\
& Clearcuts & 1.25 & 0.24 & 2 & $1.09-1.42$ \\
Vancouver island & Old growth & 3.27 & 0.05 & 3 & $3.17-3.34$ \\
\multirow{3}{*}{ Mainland South Coast } & 40-80 years old & 2.64 & 0.12 & 3 & $2.41-2.77$ \\
& Old growth & 4.38 & 0.92 & 4 & $2.98-6.98$ \\
& 40-80 years old & 3.29 & 0.30 & 4 & $2.46-3.82$ \\
& Deciduous stands & 3.91 & 0.70 & 3 & $2.72-5.14$ \\
Average & Clearcuts & 3.85 & 0.34 & 2 & $3.61-4.09$ \\
& & $\mathbf{3 . 3 8}$ & $\mathbf{0 . 3 4}$ & $\mathbf{9}$ & $\mathbf{1 . 2 5}-\mathbf{4 . 6 4}$ \\
\hline
\end{tabular}

Table 4. Breeding densities (pair $/ \mathrm{km}^{2}$ ) of waterfowl in Labrador.

\begin{tabular}{cccc}
\hline \hline & Mean & Range & $\mathrm{n}$ \\
\hline 1998 & 1.15 & $0.08-3.92$ & 31 \\
1999 & 1.45 & $0.00-4.14$ & 31 \\
\hline
\end{tabular}


APPENDIX 2. Area covered ${ }^{\dagger}$ by hydroelectric reservoirs.

Table 1. Area covered ${ }^{\dagger}$ by hydroelectric reservoirs in northern Quebec, BCR 8 (Wikipedia, September 2010).

\begin{tabular}{lc}
\hline \hline \multicolumn{1}{c}{ Reservoir } & Area $\left(\mathrm{km}^{2}\right)$ \\
\hline Gouin & 1570 \\
Dozouais & 319 \\
Manicouagan & 1942 \\
Caniapiscau & 4318 \\
Eastmain & 6682 \\
Total & 14,831 \\
\hline
\end{tabular}

${ }^{\dagger}$ this likely represent the area currently covered by reservoirs which is greater than the actual flooded areas.

Table 2. Area covered by hydroelectric reservoirs in the different BCR in Quebec (Tecsul 2009).

\begin{tabular}{ccc}
\hline \hline Bird conservation region & Area $\left(\mathrm{km}^{2}\right)$ \\
\hline 14 & 91 \\
& 13 & 292 \\
12 & 3665 \\
& 8 & 4112 \\
& 7 & 91 \\
Total & 3 & 91 \\
\hline
\end{tabular}


Table A2.1 - Distributions used to describe species-group composition, age-group breakdown and seasonal distribution of anthropogenic avian mortality for each source, used in the stochastic model to convert stage-specific losses to a total loss of potential adult breeders. When all characteristics were known, no distributions were necessary (e.g. agricultural mortality was entirely measured in loss of eggs of landbirds, and therefore there was no uncertainty in species-, age- or seasonal-breakdown).

\begin{tabular}{|c|c|c|c|c|c|c|}
\hline \multirow[b]{2}{*}{ Parameter } & \multirow[b]{2}{*}{ Distribution } & \multicolumn{2}{|c|}{$\underline{\text { Central tendency }}$} & \multicolumn{2}{|c|}{$\underline{\text { Variation or range of values }}$} & \multirow[b]{2}{*}{ Source $^{+}$} \\
\hline & & Type & Value & Type* & Values & \\
\hline \multicolumn{7}{|l|}{ Cats } \\
\hline Proportion landbirds & Uniform & Midpoint & 0.99 & Range & 0.02 & 1 \\
\hline Proportion waterbirds & Uniform & Midpoint & 0.005 & Conditional range & $0.00-0.02$ & 1 \\
\hline Proportion waterfowl & Uniform & Midpoint & 0.005 & Conditional range & $0.00-0.02$ & 1 \\
\hline Proportion of kill in fall/winter (i.e. including juveniles) & Binomial & Mean & 0.5 & Random & & 1 \\
\hline Proportion juveniles in fall & Binomial & Mean & 0.75 & Random & & 2 \\
\hline \multicolumn{7}{|l|}{ Buildings - Houses } \\
\hline Proportion juveniles in fall & Binomial & Mean & 0.75 & Random & & 2 \\
\hline Proportion of kill in fall/winter (i.e. including juveniles) & Binomial & Mean & 0.42 & Random & & 3 \\
\hline \multicolumn{7}{|l|}{ Buildings - Low- and mid-rise } \\
\hline Proportion juveniles in fall & Binomial & Mean & 0.75 & Random & & 2 \\
\hline Proportion of kill in fall/winter (i.e. including juveniles) & Uniform, binomial & Mean & 0.565 & Range, random & $0.42-0.71$ & 4 \\
\hline Proportion landbirds & Uniform & Midpoint & 0.9 & Range & 0.2 & 4 \\
\hline Proportion waterbirds & Uniform & Midpoint & 0.033 & Conditional range & $0.0-0.2$ & 4 \\
\hline Proportion waterfowl & Uniform & Midpoint & 0.033 & Conditional range & $0.0-0.2$ & 4 \\
\hline Proportion shorebirds & Uniform & Midpoint & 0.033 & Conditional range & $0.0-0.2$ & 4 \\
\hline \multicolumn{7}{|l|}{ Buildings - Tall } \\
\hline Proportion juveniles in fall & Binomial & Mean & 0.75 & Random & & 2 \\
\hline Proportion of kill in fall/winter (i.e. including juveniles) & Binomial & Mean & 0.71 & Random & & 3 \\
\hline Proportion landbirds & Uniform & Midpoint & 0.95 & Range & 0.1 & 3,5 \\
\hline Proportion waterbirds & Uniform & Midpoint & 0.0167 & Conditional range & $0.0-0.1$ & 3,5 \\
\hline Proportion waterfowl & Uniform & Midpoint & 0.0167 & Conditional range & $0.0-0.1$ & 3,5 \\
\hline Proportion shorebirds & Uniform & Midpoint & 0.0167 & Conditional range & $0.0-0.1$ & 3,5 \\
\hline
\end{tabular}


Transportation - Road vehicle collisions

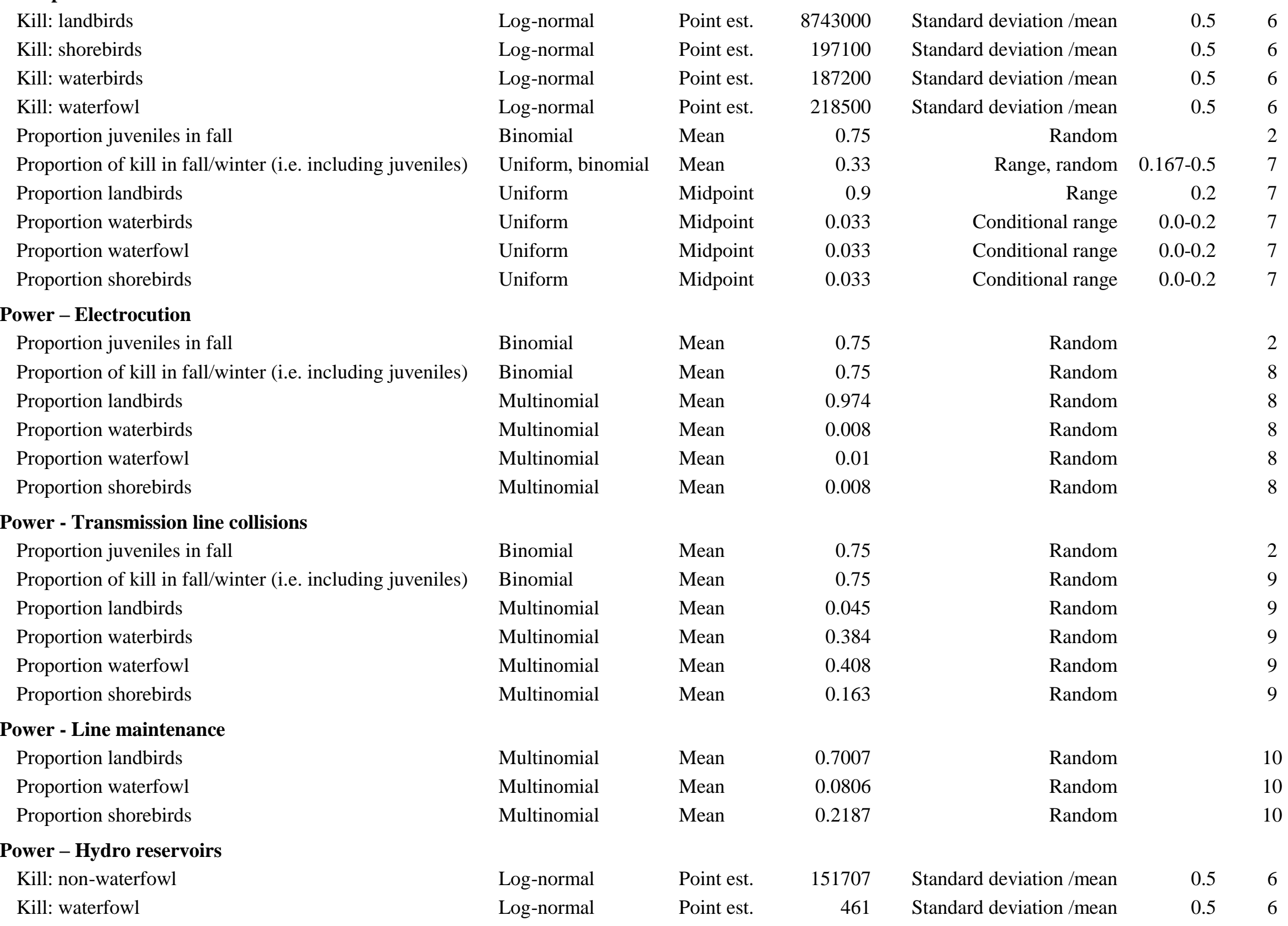


Proportion landbirds

Proportion waterbirds

Proportion shorebirds

\section{Oil and Gas - Marine - produced water}

Proportion juveniles in fall

Proportion subadults

Proportion of kill in fall/winter (i.e. including juveniles)

Oil and Gas - Marine - platform and vessel strandings

Proportion juveniles impacted

Proportion of kill in fall/winter (i.e. including juveniles)

\section{Fisheries - Marine bycatch}

Proportion adults - gill nets

Proportion of kill during breeding - gill nets

Proportion adults - long lines, otter trawls

Proportion of kill during breeding - long lines, otter trawls

\section{Power - Wind energy}

Proportion juveniles in fall

Proportion of kill in fall/winter (i.e. including juveniles)

\section{Agriculture - Pesticides}

Proportion landbirds

Proportion waterbirds

Proportion waterfowl

Proportion shorebirds

Proportion of kill during breeding

\section{Mining - Pits and quarries}

Proportion landbirds

Proportion waterbirds

Proportion shorebirds

Kill

Mining - Metals and minerals

All landbird eggs

\begin{tabular}{|c|c|c|c|c|c|}
\hline Uniform & Midpoint & 0.9 & Range & 0.2 & 7 \\
\hline Uniform & Midpoint & 0.5 & Conditional range & $0.0-0.2$ & 7 \\
\hline Uniform & Midpoint & 0.5 & Conditional range & $0.0-0.2$ & 7 \\
\hline Binomial & Mean & 0.173 & Random & & 11 \\
\hline Binomial & Mean & 0.242 & Random & & 11 \\
\hline Binomial & Mean & 0.5 & Random & & 11,12 \\
\hline Fixed & Value & 1 & None & 0 & 13 \\
\hline Fixed & Value & 1 & None & 0 & 13 \\
\hline Fixed & Value & 1 & None & 0 & 13 \\
\hline Fixed & Value & 1 & None & 0 & 13 \\
\hline Fixed & Value & 1 & None & 0 & 13 \\
\hline Uniform & Midpoint & 0.5 & Range & $0-1$ & 7 \\
\hline Binomial & Mean & 0.75 & Random & & 2 \\
\hline Uniform, binomial & Mean & 0.33 & Range, random & $0.167-0.5$ & 7 \\
\hline Uniform & Midpoint & 0.95 & Range & 0.1 & 7 \\
\hline Uniform & Midpoint & 0.0167 & Conditional range & $0.0-0.1$ & 7 \\
\hline Uniform & Midpoint & 0.0167 & Conditional range & $0.0-0.1$ & 7 \\
\hline Uniform & Midpoint & 0.0167 & Conditional range & $0.0-0.1$ & 7 \\
\hline Fixed & Value & 1 & None & 0 & 14 \\
\hline Uniform & Midpoint & 0.95 & Range & 0.2 & 7 \\
\hline Uniform & Midpoint & 0.025 & Conditional range & $0.0-0.2$ & 7 \\
\hline Uniform & Midpoint & 0.025 & Conditional range & $0.0-0.2$ & 7 \\
\hline Log-normal & Point est. & 125529 & Standard deviation /mean & 1 & 6 \\
\hline
\end{tabular}




\section{Agriculture - Haying}

All landbird eggs

\section{Transportation - Road maintenance}

Proportion landbirds

Proportion waterfowl

Proportion shorebirds

\section{Harvest - Migratory birds}

Mean

0.7007

0.0806

Multinomial

Mean

Random

Random

Mean

0.2187

Random

Proportion juveniles: ducks

Proportion juveniles: geese

Proportion juveniles: snipe and woodcock

0.735

0.30

0.515

0.515

0.515

Proportion juveniles: pigeons and doves

Proportion juveniles: murres

Proportion subadults: murres

\section{Communication - Tower collisions}

Proportion juveniles in fall

Proportion of kill in fall/winter (i.e. including juveniles)

Proportion landbirds

Proportion waterbirds

Proportion waterfowl

Proportion shorebirds

\section{Transportation - Chronic ship-source oil}

Proportion juveniles in fall

Proportion subadults

Proportion of kill in fall/winter (i.e. including juveniles)

$\begin{array}{llr}\text { Beta } & \text { Mean } & 0.735 \\ \text { Beta } & \text { Mean } & 0.30 \\ \text { Beta } & \text { Mean } & 0.515 \\ \text { Beta } & \text { Mean } & 0.515 \\ \text { Beta } & \text { Mean } & 0.515 \\ \text { Beta } & \text { Mean } & 0.5 \\ \text { Beta } & \text { Mean } & 0.3 \\ & & \\ \text { Binomial } & \text { Mean } & 0.75 \\ \text { Binomial } & \text { Mean } & 0.75 \\ \text { Multinomial } & \text { Mean } & 0.974 \\ \text { Multinomial } & \text { Mean } & 0.008 \\ \text { Multinomial } & \text { Mean } & 0.01 \\ \text { Multinomial } & \text { Mean } & 0.008 \\ & & \\ \text { Binomial } & \text { Mean } & 0.173 \\ \text { Binomial } & \text { Mean } & 0.242 \\ \text { Binomial } & \text { Mean } & 0.5\end{array}$

Standard deviation

Standard deviation

Standard deviation

Standard deviation

Standard deviation

Standard deviation

Standard deviation

$\begin{array}{rr}0.104 & 16 \\ 0.194 & 16 \\ 0.099 & 16 \\ 0.099 & 17 \\ 0.099 & 17 \\ 0.1 & 18 \\ 0.1 & 19\end{array}$

Random

Random

Random

Random

Random

Random

Random

Random

Random
15

15

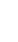

8

8

8

8

8

11

11

11,12

\footnotetext{
*Conditional ranges were values that were constrained by the requirement that the proportion of the kill assigned across species groups must sum to 1.

${ }^{+}$References for distributions: 1 - Blancher 2013; 2 - Canadian Migration Monitoring Network data from western Canada; 3 - Machtans et al. 2013; 4 - range

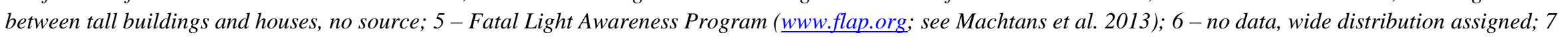

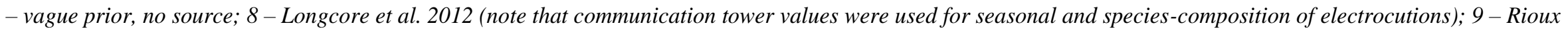

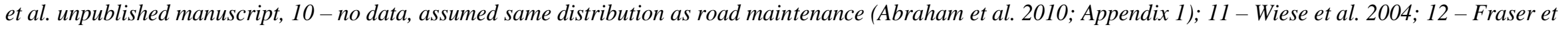

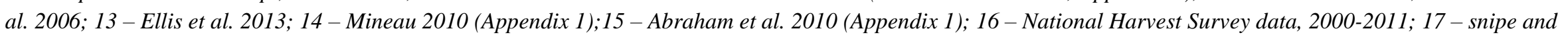
woodcock data from National Harvest Survey (2000-2011); 18 - Elliot 1991; 19 - Gaston and Robertson 2010 (band recovery data).
} 
Table A2.2 - Demographic rates used in the stochastic model for anthropogenic avian mortality, to convert stage-specific losses for each of the five major species-groups to a total loss of potential adult breeders.

\begin{tabular}{|c|c|c|c|c|c|c|}
\hline \multirow{2}{*}{ Vital rate } & \multirow[b]{2}{*}{ Distribution } & \multicolumn{2}{|c|}{ Central tendency } & \multicolumn{2}{|c|}{ Variation or range of values } & \multirow[b]{2}{*}{ Source } \\
\hline & & Type & Values & Type & Values & \\
\hline \multicolumn{7}{|l|}{ Waterfowl } \\
\hline Clutch size (C) & Uniform & Midpoint & 4.55 & Range & 1 & 1 \\
\hline Hatchability/hatch success $(\mathrm{H})$ & Beta & Mean & 0.91 & Std. deviation & 0.05 & 1 \\
\hline Nest survival/nest success $(\mathrm{N})$ & Beta & Mean & 0.13 & Std. deviation & 0.075 & 1 \\
\hline Survival to fledge $\left(\mathrm{S}_{\mathrm{y}}\right)$ & Beta & Mean & 0.39 & Std. deviation & 0.11 & 1 \\
\hline Nesting attempts (B) & Beta & Mean & 2.77 & Std. deviation & 0.25 & 1 \\
\hline Juvenile overwinter survival $\left(S_{0}\right)$ & Beta & Mean & 0.8 & Std. deviation & 0.051 & 1 \\
\hline Adult overwinter survival $\left(S_{a}\right)$ & Beta & Mean & 0.8 & Std. deviation & 0.051 & 1 \\
\hline \multicolumn{7}{|l|}{ Shorebirds } \\
\hline Fecundity $\left(C * N * H * S_{y}\right)$ & Random draws & Mean of vector & 0.357 & Values & $0.26,0.49,0.65,0.05,0.14,0.55$ & 2,3 \\
\hline Juvenile overwinter survival $\left(\mathrm{S}_{\mathrm{o}}\right)$ & Random draws & Mean of vector & 0.4095 & Values & $0.367,0.452$ & 2,3 \\
\hline Adult overwinter survival $\left(S_{\mathrm{a}}\right)$ & Random draws & Mean of vector & 0.86 & Values & $0.85,087$ & 2,3 \\
\hline \multicolumn{7}{|l|}{ Waterbirds } \\
\hline Fecundity $\left(C^{*} N^{*} H^{*} S_{y}\right)$ & Uniform & Midpoint & 1.6 & Range & 0.5 to 2.7 & 4,5 \\
\hline Juvenile overwinter survival $\left(\mathrm{S}_{\mathrm{o}}\right)$ & Beta & Mean & 0.273 & Std. deviation & $0.273 \times 0.5$ & 5 \\
\hline Adult overwinter survival $\left(\mathrm{S}_{\mathrm{a}}\right)$ & Uniform & Midpoint & 0.823 & Range & 0.727 to 0.918 & 4,5 \\
\hline \multicolumn{7}{|l|}{ Landbirds (except upland game) } \\
\hline Clutch size & Random draws & Mean / Median & $4.31 / 4.00$ & Values & (see source 6 , Appendix 1) & 6,7 \\
\hline Nest success & Random draws & Mean / Median & $0.515 / 0.463$ & Values & (see source 6 , Appendix 1) & 6,7 \\
\hline Survival to fledge $\left(S_{\mathrm{y}}\right)$ & Random draws & Mean / Median & $0.442 / 0.395$ & Values & (see source 6 , Appendix 1) & 6,7 \\
\hline Juvenile overwinter survival $\left(S_{0}\right)$ & Complementary beta & mean & 0.32 & Minimum range & 0.18 & $8^{*}$ \\
\hline Adult overwinter survival $\left(S_{\mathrm{a}}\right)$ & Complementary beta & Mean & 0.53 & Minimum range & 0.29 & 8 \\
\hline \multicolumn{7}{|l|}{ Upland game birds } \\
\hline Juvenile overwinter survival $\left(S_{o}\right)$ & Random draws & Mean of vector & 0.441 & Values & $\begin{array}{c}(0.366,0.337,0.486,0.473,0.518, \\
0.578,0.505,0.354,0.565,0.46,0.71, \\
0.279,0.014,0.38,0.51,0.48,0.48) \\
(0.366,0.337,0.486,0.473,0.518\end{array}$ & $9-13$ \\
\hline Adult overwinter survival $\left(\mathrm{S}_{\mathrm{a}}\right)$ & Random draws & Mean of vector & 0.441 & Values & $\begin{array}{c}0.578,0.505,0.354,0.565,0.46,0.71 \\
0.279,0.014,0.38,0.51,0.48,0.48)\end{array}$ & $9-13$ \\
\hline
\end{tabular}




\section{Seabirds}

Juvenile overwinter survival $\left(\mathrm{S}_{\mathrm{o}}\right)$

Beta

Beta

Mean

Adult overwinter survival $\left(\mathrm{S}_{\mathrm{a}}\right)$

Age of first breeding

Immature survival $\left(\mathrm{S}_{\mathrm{o}} * \mathrm{~S}_{\mathrm{a}}{ }^{3}\right)$
None

Uniform
Mean

Median

\subsection{2}

0.91
Std. deviation

Std. deviation

None
$0.52 \times 0.05$

$0.91 \times 0.05$

0.086-0.316

${ }^{+}$References for vital rates: 1 - Hoekman et al. 2002; 2 - Gratto-Trevor 2000; 3 - Lowther et al. 2001; 4 - Tacha et al. $1992 ; 5$ - Vennesland and Butler 2011; 6 -

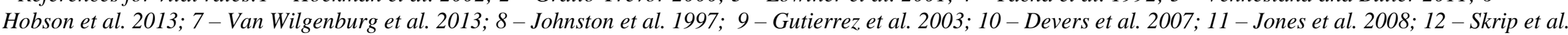
2011; 13 - Harrison 2001; 14 - Wiese et al. 2004; 15 - Huntington et al. 1996

* Estimated using the other vital rates available, assuming a stable population $\left(S_{o}=\left(1-S_{a}\right) / F\right)$, where $S_{a}$ is adult survival and $F$ is fecundity 


\section{Literature Cited (Appendix 2)}

Blancher, P. J. 2013. Estimated number of birds killed by house cats (Felis catus) in Canada. Avian Conservation and Ecology (this issue).

Devers, P. K., D. F. Stauffer, G. W. Norman, D. E. Steffen, D. M. Whitaker, J. D. Sole, T. J. Allen, S. L. Bittner, D. A. Buehler, J. W. Edwards, D. E. Figert, S. T. Friedhoff, W. W. Giuliano, C. A. Harper, W. K. Igo, R. L. Kirkpatrick, M. H. Seamster, H. A. Spiker Jr., D. A. Swanson, and B. C. Tefft. 2007. Ruffed grouse population ecology in the Appalachian Region. Wildlife Monograph 168:1-36.

Elliot, R.D. 1991. The management of the Newfoundland turr hunt. Pages in 29-35 A.J. Gaston and R.D. Elliot, editors. Studies of High-Latitude Seabirds 2: Conservation Biology of ThickBilled Murres in the Northwest Atlantic. Canadian Wildlife Service, Ottawa, Ontario, Canada.

Ellis, J. I., S. I. Wilhelm, A. Hedd, G. S. Fraser, G. J. Robertson, J.-F. Rail, M. Fowler, and K. H. Morgan. 2013. Mortality of migratory birds from marine commercial fisheries and offshore oil and gas production in Canada. Avian Conservation and Ecology (this issue).

Fraser, G., J. Russell, and W. Von Zharen. 2006. Produced water from offshore oil and gas installations on the Grand Banks, Newfoundland and Labrador: Are the potential effects of seabirds sufficiently known. Marine Ornithology 34:147-156.

Gaston, A. J., and G. J. Robertson. 2010. Trends in the harvest of Brünnich's Guillemots in Newfoundland: Effects of regulatory changes and winter sea-ice conditions. Wildlife Biology 16:47-55.

Gratto-Trevor, C. L. 2000. Marbled Godwit (Limosa fedoa). In A. Poole, editor. The Birds of North America Online. Birds of North America, Inc. [online] URL: http://bna.birds.cornell.edu/bna/species/492

Gutierrez, R.J., G. S. Zimmerman, and G.W. Gullion. 2003. Daily survival rates of ruffed grouse Bonasa umbellus in northern Minnesota. Wildlife Biology 9: 351-356.

Harrison, S. 2001. Effects of forest connectivity on ecological processes: using spruce grouse as a model system. Dissertation. University of British Columbia, Vancouver, British Columbia, Canada.

Hobson, K. A., A. Wilson, S. L. Van Wilgenburg, and E. Bayne. 2013. An estimate of nest loss in Canada due to industrial forestry operations. Avian Conservation and Ecology (this issue). 
Hoekman, S.T., L. S. Mills, D. W. Howerter, J.H. Devries, and I. J. Ball. 2002. Sensitivity analyses of the life cycle of Midcontinent Mallards. Journal of Wildlife Management 66:883900.

Huntington, C. E., R. G. Butler, and R. A. Mauck. 1996. Leach's Storm-Petrel (Oceanodroma leucorhoa). In A. Poole, editor. The Birds of North America Online. Birds of North America, Inc. [online] URL: http://bna.birds.cornell.edu/bna/species/233

Johnston, J. P., W. J. Peach, R. D. Gregory, and S. A. White. 1997. Survival rates of tropical and temperate passerines: A Trinidadian perspective. American Naturalist 150: 771-789.

Jones, B. C., C. L. Dobey, J. L. Kleitch, C.A. Harper, D.A. Buehler, W.G. Minser III, and G.S. Warburton. 2008. Ruffed Grouse Survival and Population Structure in Western North Carolina. Proceedings of the Annual Conference of the Southeast Association of Fish and Wildlife Agencies 62:51-57.

Longcore, T., C. Rich, P. Mineau, B. MacDonald, D. G. Bert, L. M. Sullivan, E. Mutrie, S. A. Gauthreaux Jr., M. L. Avery, R. L. Crawford, A. M. Manville II, E. R. Travis, and D. Drake. 2012. An estimate of avian mortality at communication towers in the United States and Canada. PLOS ONE 7(4): e34025. URL: doi:10.1371/journal.pone.oo34025

Lowther, P. E., H. D. Douglas III, and C. L. Gratto-Trevor. 2001. Willet (Tringa semipalmata). In A. Poole, editor. The Birds of North America Online. Birds of North America, Inc. [online] URL: http://bna.birds.cornell.edu/bna/species/579

Machtans, C. S., C. H. R. Wedeles, and E. M. Bayne. 2013. A first estimate for Canada of the number of birds killed by colliding with buildings. Avian Conservation and Ecology (this issue).

Skrip, M. M., W. F. Porter, B. L. Swift, and M. V. Schiavone 2011. Fall-winter survival of Ruffed Grouse in New York State. Northeastern Naturalist 18:395-410. 2011.

Tacha, T. C., S. A. Nesbitt, and P. A. Vohs. 1992. Sandhill Crane (Grus canadensis). In A. Poole, editor. The Birds of North America Online. Birds of North America, Inc. [online] URL: http://bna.birds.cornell.edu/bna/species/031

Van Wilgenburg, S. L., K. A. Hobson, E. M. Bayne, and N. Koper. 2013. Estimated avian nest loss associated with oil and gas exploration and extraction in the Western Canadian Sedimentary Basin. Avian Conservation and Ecology (this issue). 
Vennesland, R. G., and R. W. Butler. 2011. Great Blue Heron (Ardea herodias). In A. Poole, editor. The Birds of North America Online. Birds of North America, Inc. [online] URL: http://bna.birds.cornell.edu/bna/species/025

Wiese, F. K., G. J. Robertson, and A. J. Gaston. 2004. Impacts of chronic marine oil pollution and the murre hunt in Newfoundland on thick-billed murre Uria lomvia populations in the eastern Canadian Arctic. Biological Conservation 116: 205-216. 
Appendix 3. Details of population-level impacts of anthropogenic avian mortality in Canada.

Table A3.1 -Total mortality relative to abundance for species (or regional populations) and families, as illustrated in Fig. 2. Original stage-specific mortality totals presented in each paper are listed by life stage ( Ke: eggs/nestlings, $K$-unk: unknown-age mobile individuals; $K f$ : fledglings; $K a$ : adults), as well as the total converted mortality in equivalent number of adult breeders is also shown $(M)$. The proportion value in the final column (\%) represents the ratio between $\mathrm{M}$ and abundance. Abundance estimates are those provided directly by authors, except for building collisions, where family-level abundance was derived from current Canadian estimates (Blancher 2002, P. Blancher unpubl. data), and used to estimate proportional kill (M / abundance).

\begin{tabular}{|c|c|c|c|c|c|c|c|c|c|}
\hline Source & Species or family (region & & Group & Ke & K-unk. & $K f \quad K a$ & $M$ & Abundance & $\%$ \\
\hline Marine bycatch & Common Murre (Funk Is.) & Uria aalge & Seabirds & - & - & $-4,500$ & 4,500 & 984,000 & $0.46 \%$ \\
\hline Marine bycatch & Black-footed Albatross & Phoebastria nigripes & Seabirds & - & - & 99 & 99 & 2,500 & $3.96 \%$ \\
\hline Marine bycatch & Common Eider (SW NS) & Somateria mollissima & Seabirds & - & - & -532 & 532 & 7,600 & $7.00 \%$ \\
\hline Marine bycatch & Northern Gannet & Morus bassanus & Seabirds & - & - & $-\quad 320$ & 320 & 160,000 & $0.20 \%$ \\
\hline Marine bycatch & Great Shearwater & Puffinus gravis & Seabirds & - & - & $-2,346$ & 2,135 & $1,500,000$ & $0.14 \%$ \\
\hline Oil and gas - marine & Leach's Storm-Petrel & Oceanodroma leucorhoa & Seabirds & - & - & 62 & 12 & $20,000,000$ & $0.00 \%$ \\
\hline Oil and gas - marine & Thick-billed Murre & Uria lomvia & Seabirds & - & 126 & - & 71 & $8,000,000$ & $0.00 \%$ \\
\hline Oil and gas - marine & Common Murre & Uria aalge & Seabirds & - & 32 & - & 18 & $2,000,000$ & $0.00 \%$ \\
\hline Oil and gas - marine & Dovekie & Alle alle & Seabirds & - & 2,086 & - & 1,157 & $132,000,000$ & $0.00 \%$ \\
\hline Oil and gas - terrestrial & Sprague's Pipit & Anthus spragueii & Landbirds & - & - & 482 & 482 & 613,000 & $0.08 \%$ \\
\hline Oil and gas - terrestrial & Western Meadowlark & Sturnella neglecta & Landbirds & - & - & 550 & 550 & $2,100,000$ & $0.03 \%$ \\
\hline Oil and gas - terrestrial & Canada Warbler & Cardellina canadensis & Landbirds & - & - & 74 & 74 & 210,000 & $0.04 \%$ \\
\hline Oil and gas - terrestrial & Ovenbird & Seiurus aurocapilla & Landbirds & - & - & $\begin{array}{l}-730 \\
\end{array}$ & 730 & $1,750,000$ & $0.04 \%$ \\
\hline Wind energy & Horned Lark & Eremophilia alpestris & Landbirds & - & 1,480 & - & 1,171 & $30,000,000$ & $0.00 \%$ \\
\hline
\end{tabular}




\begin{tabular}{|c|c|c|c|c|c|c|c|c|c|c|}
\hline Wind energy & Golden-crowned Kinglet & Regulus satrapa & Landbirds & - & 888 & - & - & 702 & $23,000,000$ & $0.00 \%$ \\
\hline Wind energy & Red-eyed Vireo & Vireo olivaceus & Landbirds & - & 888 & - & - & 702 & $96,000,000$ & $0.00 \%$ \\
\hline Wind energy & European Starling & Sturnus vulgaris & Landbirds & - & 740 & - & - & 585 & $30,000,000$ & $0.00 \%$ \\
\hline Wind energy & Tree Swallow & Tachycineta bicolor & Landbirds & - & 592 & - & - & 468 & $12,000,000$ & $0.00 \%$ \\
\hline Wind energy & Canada Warbler & Cardellina canadensis & Landbirds & - & 44 & - & - & 35 & $1,350,000$ & $0.00 \%$ \\
\hline Wind energy & Chimney Swift & Chaetura pelagica & Landbirds & - & 44 & - & - & 35 & 145,000 & $0.02 \%$ \\
\hline Agriculture (haying) & Bobolink & Dolichonyx oryzivorus & Landbirds & 666,784 & - & - & - & 48,570 & $3,991,300$ & $1.22 \%$ \\
\hline Agriculture (haying) & Savannah Sparrow & Passerculus sandwichensis & Landbirds & 940,697 & - & - & - & 68,522 & $23,628,000$ & $0.29 \%$ \\
\hline Agriculture (haying) & Clay-coloured Sparrow & Spizella pallida & Landbirds & 215,321 & - & - & - & 15,684 & $20,174,600$ & $0.08 \%$ \\
\hline Agriculture (haying) & Vesper Sparrow & Pooecetes gramineus & Landbirds & 249,074 & - & - & - & 18,143 & $10,012,100$ & $0.18 \%$ \\
\hline Agriculture (haying) & Horned Lark & Eremophila alpestris & Landbirds & 137,524 & - & - & - & 10,017 & $11,476,240$ & $0.09 \%$ \\
\hline Road maintenance & Killdeer & Charadrius vociferous & Shorebirds & 2,026 & - & - & - & 74 & $1,613,200$ & $0.00 \%$ \\
\hline Road maintenance & Savannah Sparrow & Passerculus sandwichensis & Landbirds & 9,306 & - & - & - & 703 & $35,770,000$ & $0.00 \%$ \\
\hline Road maintenance & Song Sparrow & Melospiza melodia & Landbirds & 4,175 & - & - & - & 316 & $24,030,000$ & $0.00 \%$ \\
\hline Road maintenance & Clay-colored Sparrow & Spizella pallida & Landbirds & 1,088 & - & - & - & 82 & $18,982,000$ & $0.00 \%$ \\
\hline Road maintenance & Vesper Sparrow & Pooecetes gramineus & Landbirds & 3,053 & - & - & - & 231 & $10,692,600$ & $0.00 \%$ \\
\hline Road maintenance & Mallard & Anas platyrhynchos & Waterfowl & 5,501 & - & - & 149 & 352 & $3,857,800$ & $0.01 \%$ \\
\hline Mining - Pits/quarries & Killdeer (ON/QC/BC/AB) & Charadrius vociferus & Shorebirds & 6,725 & - & - & - & 246 & $1,230,000$ & $0.02 \%$ \\
\hline Mining - Pits/quarries & Bank Swallow (ON/QC/BC/AB) & Riparia riparia & Landbirds & 66,573 & - & - & - & 5,031 & $1,540,000$ & $0.33 \%$ \\
\hline Buildings - Houses & Hawks & Accipitridae & Landbirds & - & 6,207 & - & - & 48,758 & $2,622,622$ & $1.86 \%$ \\
\hline
\end{tabular}




\begin{tabular}{|c|c|c|c|c|c|c|c|c|c|c|}
\hline Buildings - Houses & Pigeons & Colombidae & Landbirds & - & 573,793 & - & - & 422,570 & $7,740,543$ & $5.46 \%$ \\
\hline Buildings - Houses & Hummingbirds & Trochilidae & Landbirds & - & 220,690 & - & - & 162,527 & $10,227,596$ & $1.59 \%$ \\
\hline Buildings - Houses & Woodpeckers & Picidae & Landbirds & - & 286,897 & - & - & 211,285 & $66,624,318$ & $0.32 \%$ \\
\hline Buildings - Houses & Jays and crows & Corvidae & Landbirds & - & 331,034 & - & - & 243,790 & $39,786,259$ & $0.61 \%$ \\
\hline Buildings - Houses & Chickadees & Paridae & Landbirds & & $2,891,034$ & - & - & $2,129,102$ & $48,613,670$ & $4.38 \%$ \\
\hline Buildings - Houses & Nuthatches & Sittidae & Landbirds & - & 640,000 & - & - & 471,328 & $19,349,207$ & $2.44 \%$ \\
\hline Buildings - Houses & Robins and thrushes & Turdidae & Landbirds & - & $3,553,103$ & - & - & $2,616,683$ & $333,229,833$ & $0.79 \%$ \\
\hline Buildings - Houses & Warblers & Parulidae & Landbirds & - & 573,793 & - & - & 422,570 & $1,342,494,691$ & $0.03 \%$ \\
\hline Buildings - Houses & Sparrows & Emberizidae & Landbirds & - & $7,260,690$ & - & - & $5,347,135$ & $913,357,792$ & $0.59 \%$ \\
\hline Buildings - Low/mid & Warblers & Parulidae & Landbirds & - & 508,800 & - & - & 328,411 & $1,342,494,691$ & $0.02 \%$ \\
\hline Buildings - Low/mid & Sparrows & Emberizidae & Landbirds & 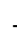 & 422,400 & - & - & 272,643 & $913,357,792$ & $0.03 \%$ \\
\hline Buildings - Low/mid & Robins and thrushes & Turdidae & Landbirds & - & 355,200 & - & - & 229,268 & $333,229,833$ & $0.07 \%$ \\
\hline Buildings - Low/mid & Cardinals & Cardinalidae & Landbirds & - & 151,200 & - & - & 97,594 & $14,699,849$ & $0.66 \%$ \\
\hline Buildings - Low/mid & Finches & Fringillidae & Landbirds & 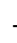 & 96,000 & - & - & 61,964 & $62,185,839$ & $0.10 \%$ \\
\hline Buildings - Low/mid & Mimids & Mimidae & Landbirds & - & 96,000 & - & - & 61,964 & $7,284,285$ & $0.85 \%$ \\
\hline Buildings - Tall & Warblers & Parulidae & Landbirds & - & 17,688 & - & - & 9,808 & $1,342,494,691$ & $0.00 \%$ \\
\hline Buildings - Tall & Sparrows & Emberizidae & Landbirds & - & 15,745 & - & - & 8,730 & $913,357,792$ & $0.00 \%$ \\
\hline Buildings - Tall & Robins and thrushes & Turdidae & Landbirds & - & 4,221 & - & - & 2,340 & $333,229,833$ & $0.00 \%$ \\
\hline Buildings - Tall & Creepers & Certhiidae & Landbirds & - & 2,278 & - & - & 1,263 & $16,106,293$ & $0.01 \%$ \\
\hline Buildings - Tall & Chickadees & Paridae & Landbirds & 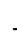 & 2,211 & - & - & 1,226 & $48,613,670$ & $0.00 \%$ \\
\hline
\end{tabular}




\section{Literature Cited (Appendix 3)}

Blancher, P. J. 2002. Importance of breeding birds exported from Canada - information on landbirds and shorebirds. Unpublished report to the North American Bird Conservation Initiative Canada National Council, Ottawa, Ontario, Canada. 
Appendix 4. Methodology for maps of anthropogenic avian mortality risk.

\section{Overview}

The two maps shown in main manuscript were created by assembling vector format data layers and overlaying a $50 \times 50 \mathrm{~km}$ tile grid for summarizing the data. We calculated the relative proportion of each source of mortality occurring in each grid square as a function of direct or indirect measures of intensity of each mortality source. The estimated kill for each source was then allocated across the applicable grid tile according to the proportional intensity of the mortality source calculated. The tile grid was used to summarize the overall bird mortalities occurring across Canada based on the following seven data layers representing eight sources of terrestrial mortality:

- Roads

- Communication Towers

- Terrestrial Oil and Gas

- Forestry

- Agriculture

- Population Density (used as an index of building and cat distribution)

- Wind Farms.

These were the only sources for which suitable data were available for mapping. A detailed summary of data sources, manipulations, and a map for each mortality source is provided in this appendix. All work was done in ArcGIS 10.0 and area calculations were done in Albers Equal Area Conic projection. Specific procedures/functions are noted to encourage replication or improvement.

The general approach for each layer followed this example: The total amount of disturbance for each mortality source was calculated for all of Canada (e.g. $\mathrm{km}$ of roads or $\mathrm{km}^{2}$ of oil and gas disturbance). Grid tiles overlapping that mortality source were identified. For instance, if 137 grid tiles had oil and gas activity, the total area of oil and gas disturbance was taken from the previous calculation and the proportion of disturbance in each tile was derived by simple division. The proportional kill for that entire mortality source was then assigned to each applicable tile. If a single tile had $5 \%$ of the total national disturbance, the total kill for oil and gas was multiplied by 0.05 and assigned to that tile. Therefore the units for each mortality source are in birds or nests taken as per the original research paper. Standardized values from our manuscript Table 3 were not used because they were not calculated at the same geographic level of specificity (e.g. kills for agriculture were converted nationally, not at the BCR level).

Grid totals (number of kills) were created by summing the values for the eight mortality sources above. Each map was coloured in a colour ramp of 10 classes and the natural breaks (Jenks) classification method was applied for all datasets (http://en.wikipedia.org/wiki/Jenks_natural_breaks_optimization). Note that this classifier does not produce equal width classes, but does optimize the splits for presentation.

As noted generally in the manuscript text, the following caveats apply:

- The map is a gross approximation only and we acknowledge that none of the sources/sectors above would be expected to have kill exactly proportional to their intensity. Technically we 
consider it to be a map of potential risk that remains unrealized until the sources of mortality interact with birds and result in deaths.

- Actual kill in any given tile would depend on many factors that cannot be mapped with currently available national data: the density of birds, the specific interaction of the source/sector with those birds in each environment (i.e. traffic volume, height of roadside verges, local features attracting birds), the seasonal timing of the activity, and even local weather conditions in any given year.

- In certain cases, such as forestry, a realistic calculation should be based on the location of actual cutting in Canada rather than the entire area available for cutting. This would be similar for farming; the location and spatially explicit footprint of each crop or farm type would ideally be known nationally. Such data are not available.

- In spite of these caveats, it remains self-evident that kills occur where the source/sector operates and, as a generalization, more activity by a given source/sector would result in some incremental increase in kill. We have constrained the scale of the map and smoothed the final product to minimize the perceived precision of the mapping classes.

Each section below contains the data source for each source of mortality, how we derived the maps and sector-specific maps that are not presented in the main paper.

\section{Bird-vehicle Collisions}

- Source: GeoBase website (http://www.geobase.ca/) National Roads Database, 2011.

- Spatial Reference: NAD83CSRS (North American Datum 1983 in Canadian Spatial Reference System)

- Ellipsoid Name: GRS80 (Geodetic Reference System 1980)

- Total kill figure used: Kills per km/day of road, by ecotype, from Table 3 in Bishop and Brogan 2013.

The National Road Network (NRN) is produced, updated, and distributed (with the support of GeoConnections) through collaborative agreements between Natural Resources Canada, Statistics Canada, and the provincial and territorial governments. A detailed summary of the product is here:

\section{http://www.geobase.ca/geobase/en/data/nrn/description.html}

\section{Methodology:}

A roads layer was created from the NRN which displayed only 1 and 2 lane paved roads that occurred outside of urban areas using a 'select by attributes' query and did not contain Ramp features. A 'create layer from selected features' was then used to create a new layer containing only these selected features. The query used to extract this data was: ("NBRLANES" $<3$ AND ("PAVSTATUS" = '1Paved' OR "PAVSTATUS" = '2Paved' OR "PAVSTATUS" = 'Paved' ) AND "ROADCLASS" <> 'Ramp').

Roads were separated by ecozone type following the methods in Bishop and Brogan (2013); ecozone types consisted of: Broadleaf and Mixed Forest (classified as one ecozone), Coniferous High Volume (1\%), Coniferous Low Volume (99\%), Cropland, 90\% Rangeland Area, 10\% Rangeland Area and Wetlands. Each ecozone type was assigned a kill value for the number of birds killed per kilometre of road based on Table 3 in Bishop and Brogan (2013). 
Roads were separated into each individual $50 \mathrm{~km}$ tile by using the 'Intersect' tool. The intersect output contained numerous road segments and types by tile; each ecotype total was multiplied by the value for birds killed per kilometre per day corresponding to the ecotype classification of that segment and then multiplied by the number of days (122) and adjusted for scavenging by multiplying by 2.97 (Table 3, Bishop and Brogan 2013).

The 'merge' tool was then used to join all 7 ecozone types together with all of their final road values for the number of birds killed by unique tile ID. The 'Dissolve' tool was then run to dissolve all roads by Tile ID and sum the total number of birds killed annually for all 7 ecozones.

The result of the road analysis is shown in Fig. A4.1.

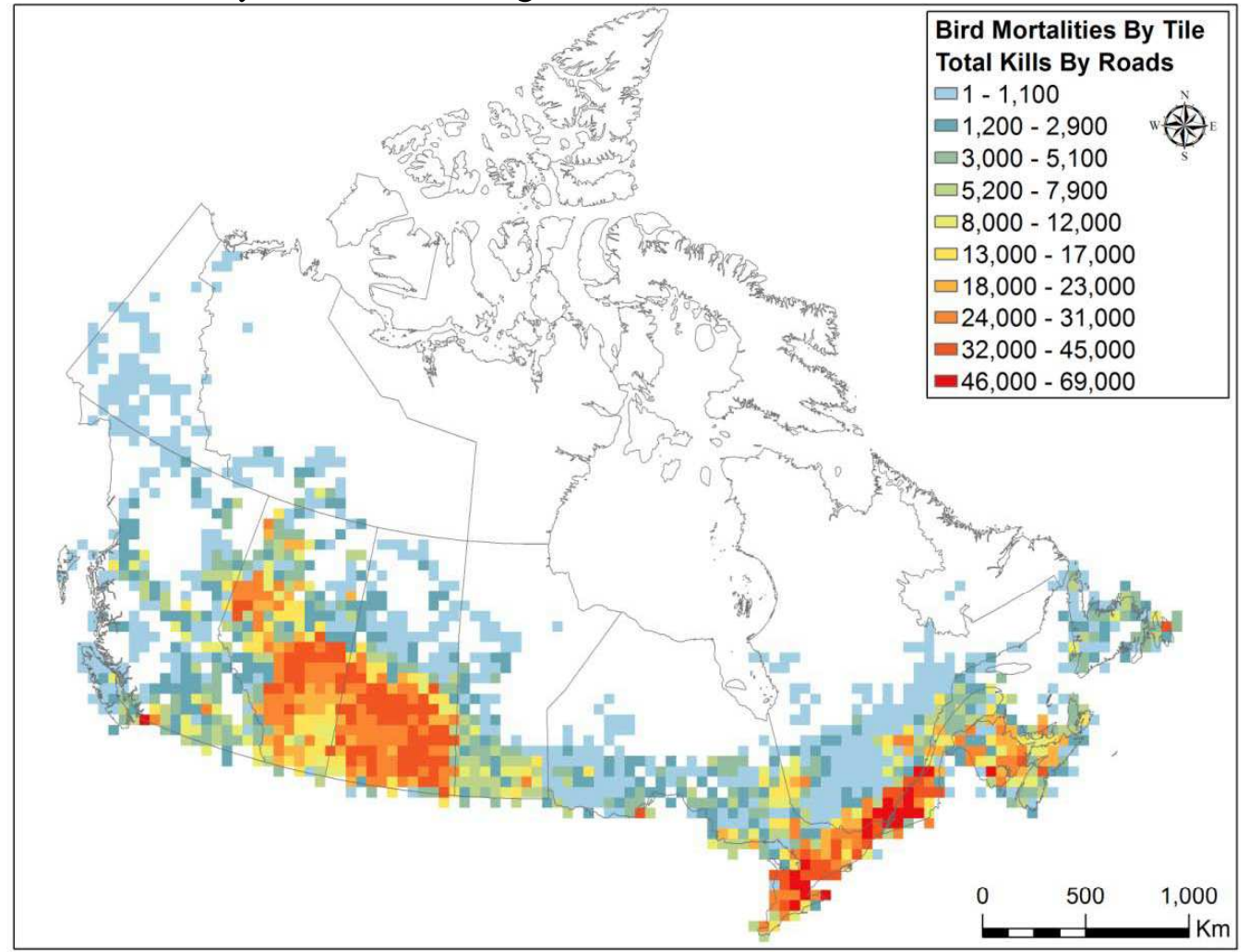

Fig. A4.1 - Approximated distribution of bird-vehicle collisions in Canada on road types considered by Bishop and Brogan (2013).

\section{Communication Towers}

- Source: NAVCAN database of tower locations. License paid for by Environment Canada for EC use as specified in agreement. Substantial corrections on the original data were performed by Beau MacDonald (The Urban Wildlands Group, Los Angeles, CA, USA) for use in Longcore et al. (2012).

- Spatial Reference: $n / a$

- Ellipsoid Name: $\mathrm{n} / \mathrm{a}$

- Total kill figure used: 221,000 (Table 7, Longcore et al. 2012). 


\section{Methodology}

NavCan tower data were provided in a proofread and cleaned format. The equation from Fig. 3 of Longcore et al. (2012) was used to calculate predicted tower mortality for each tower. Longcore et al. (2012) used a probability-based reduction of totals at the BCR level to account for towers that were guyed or not and steadily lit or not. We applied a BCR-specific static adjustment to each tower kill value such that our BCR total matched those from Longcore et al (2012) because we calculated mortality at the individual tower level. Only towers $>60 \mathrm{~m}$ tall were considered in our analysis, the same restriction used by Longcore et al. (2012).

The $50 \times 50 \mathrm{~km}$ tile grid was overlaid with the communications tower point layer and a spatial join was performed to link each point to the proper tile that it fell within. The Dissolve tool was then run to sum the number of kills by tile ID using the statistics function.

The results of our communication towers analysis is shown in Fig. A4.2

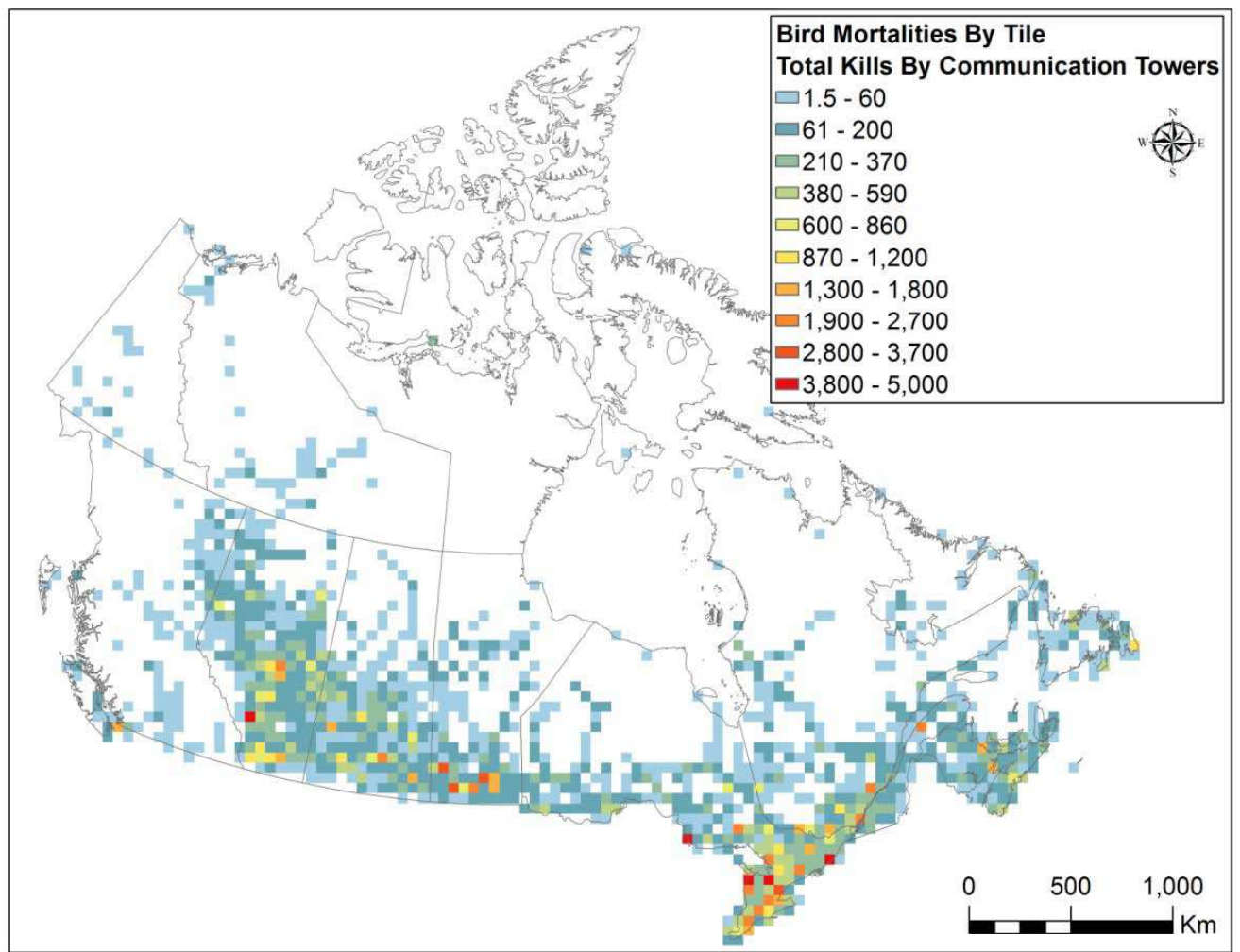

Fig. A4.2 - Approximated distribution of kills from birds colliding with communication towers $>60 \mathrm{~m}$ in Canada.

\section{Terrestrial Oil and Gas (wells, seismic lines, pipelines)}

Total kill figures are from the mean value column of Table 3 (number of nests) in Van Wilgenburg et al. (2013). Those data are split in each province into boreal areas and prairie areas.

\section{Manitoba}


- Source: Manitoba Innovation, Energy and Mines http://www.gov.mb.ca/iem/petroleum/gis/index.html

- Spatial Reference: North American 1983 CSRS UTM Zone 14N

- Ellipsoid Name: GRS 80

- Total kill figure used: Boreal: 211, Prairie: 92

Metadata are not provided with the wells file on the above noted website.

The data was displayed by township (6 mile by 6 mile square area polygons) which contained the number of wells within each township.

\section{Saskatchewan}

- Source: Saskatchewan Energy and Resources oil and gas information viewer, http://www.infomaps.gov.sk.ca/website/SIR_Oil_And_Gas_Wells/viewer.htm

- Spatial Reference: North American 1983 UTM Zone 13N

- Ellipsoid Name: GRS 80

- Total kill figure used: Boreal: 284, Prairie: 2602

Metadata are available at https://www.geosask.ca by entering the Spatial Gallery, searching for the data layer "wells" and using the metadata viewer.

The data was displayed by township (6mile by 6 mile squares) which contained the number of wells within each township.

\section{Alberta}

- Source: Energy Resources Conservation Board (http://www.ercb.ca/) data subscription for well data to Environment Canada.

- Spatial Reference: North American 1983 UTM Zone 10N

- Ellipsoid Name: GRS 80

- Total kill figure used: Boreal: 1125, Prairie: 5677

The data were in point format and each point contained a value for the number of wells at that specific location.

Metadata are provided via Environment Canada's digital subscription (i.e. not on the ERCB website) and are available by contacting the authors.

\section{British Columbia}

- Source: British Columbia Oil and Gas Commission, ftp://www.bcogc.ca/outgoing/OGC_Data/Wells/

- Spatial Reference: BC Albers, NAD83

- Ellipsoid Name: GRS 80

- Total kill figure used: Boreal: 1125, Prairie: n/a

In addition to the short metadata associated with the above link, more information on the data is available here: ftp://www.bcogc.ca/outgoing/OGC_Data/ePASS_Documents/ePASS_Standards_Requirements.pdf 


\section{Methodology}

We restricted our analysis to the area covered by Van Wilgenburg et al. (2013). Raw well data for Manitoba and Saskatchewan were processed by S. Van Wilgenburg and overlaid with the official township grid for those provinces. The township grid (polygon data) for Manitoba and Saskatchewan were placed into one dataset by using the 'Merge' tool to place all townships into one dataset (containing the number of wells by township).

No township data was available without an associated fee for British Columbia and Alberta so a $6 \times 6$ mile tiled grid was created using the Fishnet tool in order to represent the size of townships within Alberta and British Columbia (Taylor 1975). The $6 \times 6$ mile tiled grid was then overlaid with the point layer that was provided for $\mathrm{AB}$ and $\mathrm{BC}$ in order to assign a well count value to each township grid using a spatial join. The output was a polygon vector that contained numerous point data (many points) to one township. The dissolve tool was used to sum up the total count value of wells by township. The township polygon for $\mathrm{AB}$ and $\mathrm{BC}$ was then added to the $\mathrm{MB}$ and SK township dataset using the 'Merge' tool.

Using the Bird Conservation Regions (BCR) layer (http://www.nabci-us.org/bcrs.htm) the townships were classified as Boreal or Prairie based on which larger proportion of the township fell within the specified BCR (11 for prairie, 6 for boreal).

We calculated the area in each township disturbed by seismic line and pipelines using the following formulas from Appendix 1 of Van Wilgenburg et al. (2013) based on the well count value.

- The percent of township disturbed by seismic lines in the Boreal Plain Ecozone can be estimated as: $\%=(0.974+0.807 * \log (\text { wells }))^{2}$

- The percent of a township disturbed by seismic lines in the Grassland Natural Region (Prairie) can be estimated as: $\%=(0.430+0.006 * \log (\text { wells }))^{2}$

- The percent of a township disturbed by pipeline right-of-ways in the Boreal Plain Ecozone can be estimated as: $\%=(0.699+1.866 * \log (\text { wells }))^{2}$

- The percent of a township disturbed by pipeline right-of ways in the Grassland Natural Region (Prairie) can be estimated as: $\%=(0.655+1.811 * \log (\text { wells }))^{2}$

We had to convert well count to well area within each township. A value of $20,000 \mathrm{~m}^{2}$ ( 2 ha) was assigned to each well based on written advice from the Canadian Association of Petroleum Producers as used in Van Wilgenburg et al. (2013). The sum of the area disturbance by seismic lines, pipelines and the total well areas by township were summed to obtain the overall area of disturbance by township.

Townships were intersected with the $50 \times 50 \mathrm{~km}$ tile grid. An area calculation was done on the divided (intersected) townships in order to determine the proportion of that township that fell within each tile. The proportion value was then multiplied by the overall area of disturbance by township (sum of Seismic, Pipeline and Well area) in order to determine the total area of disturbance within each tile. The 'Dissolve' tool was run to dissolve all townships by tile and the statistics field was used to 'SUM' the area of disturbance values for each portion of the townships that fell within each individual tile.

The BCR layer was then overlaid with the $50 \times 50 \mathrm{~km}$ tiled disturbance area layer and each tile was reclassified as 'Boreal' or 'Prairie'. Whichever larger portion $(>50 \%)$ of the cell fell within the specified BCR then that tile was classified as that region. 
The proportion value for each tile was calculated from the final disturbance value depending on which province and part of a province (boreal or prairie) the tile fell into. Total disturbance value by each provincial region was calculated. In areas where tiles overlapped with provinces and/or prairie/boreal regions, the proportion of each had to be calculated using the area field and multiplying those proportions based off of the total tile area of $2500 \mathrm{~km}^{2}$; in all other cases the tile had a value of $100 \%$ for the province/region intersect.

The proportion of each $50 \times 50 \mathrm{~km}$ tile disturbed were then multiplied by the kill values listed above for each of the 7 intersections of province and habitat. Calculations were all performed within the Field Calculator.

The result of our oil and gas analysis is shown in Fig. A4.3.

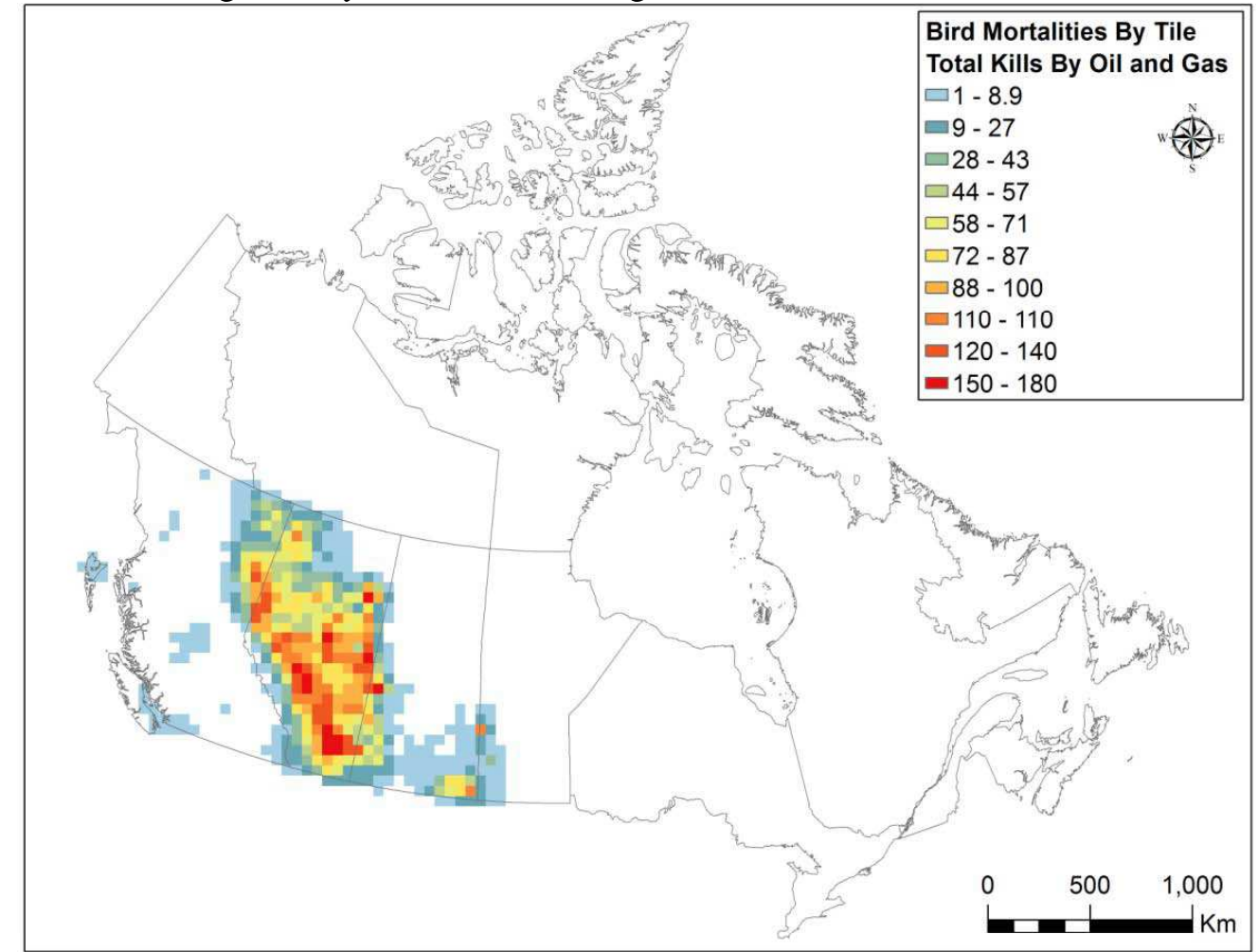

Fig. A4.3 - Approximated distribution of nests destroyed from the oil and gas sector in the western Canadian sedimentary basin as enumerated by Van Wilgenburg et al. (2013).

\section{Forestry}

- Source: Global Forest Watch Canada data warehouse (http://datawarehouse.globalforestwatch.ca/ ), Canadian Commercial Forest Tenures Map (last updated on January $30^{\text {th }}, 2004$ )

- Spatial Reference: Lambert Conformal Conic

- Ellipsoid Name: Clarke 1866

- Total kill figures (nests) from Hobson et al. (2013, Table 6) by averaging the low PIF estimate and the high BAM estimate (see Table A4.1) 
A brief description of the data from Global Forest Watch Canada is: "A compilation of the basic forest tenure/concession data from all of the provinces. [It] includes companies in order of allowable annual cut (where applicable/available). Tenures/concession polygons will contain areas restricted to logging and other resources extraction activities by protected areas legislation and by other legislative and policy restrictions. We attempted to include only crown lands and exclude all private lands where the data was available (e.g., New Brunswick), but other jurisdictions may have inadvertently included private lands."

Metadata: http://www.globalforestwatch.ca/data/tenure_spatial/CANADA/canada tenures.txt

Table A4.1 - Estimated nests destroyed by forestry from Hobson et al. (2013).

\begin{tabular}{cc}
\hline \hline Province & Estimated nests destroyed \\
\hline BC & 387,926 \\
AB & 62,210 \\
SK & 15,316 \\
MB & 6039 \\
ON & 255,832 \\
QC & 372,680 \\
NB & 126,316 \\
NS & 88,924 \\
PEI & 6938 \\
NF & 15,576 \\
NT, YK, NU & 0 (no forest tenure map data available, \\
& no tenures, or no forestry in the region) \\
\hline
\end{tabular}

\section{Methodology}

The forestry tenure data (Fig. A4.4) was dissolved and overlaid with the $50 \times 50 \mathrm{~km}$ tile grid and clipped by tile. Tiles were broken down by province and the proportion of forestry tenure that occurred within each tile, within a province was determined. The proportion of forestry by tile by province was then multiplied by the kill values (above) by province in order to determine the total kills by tile. This value represents the number of nests destroyed by tile. 


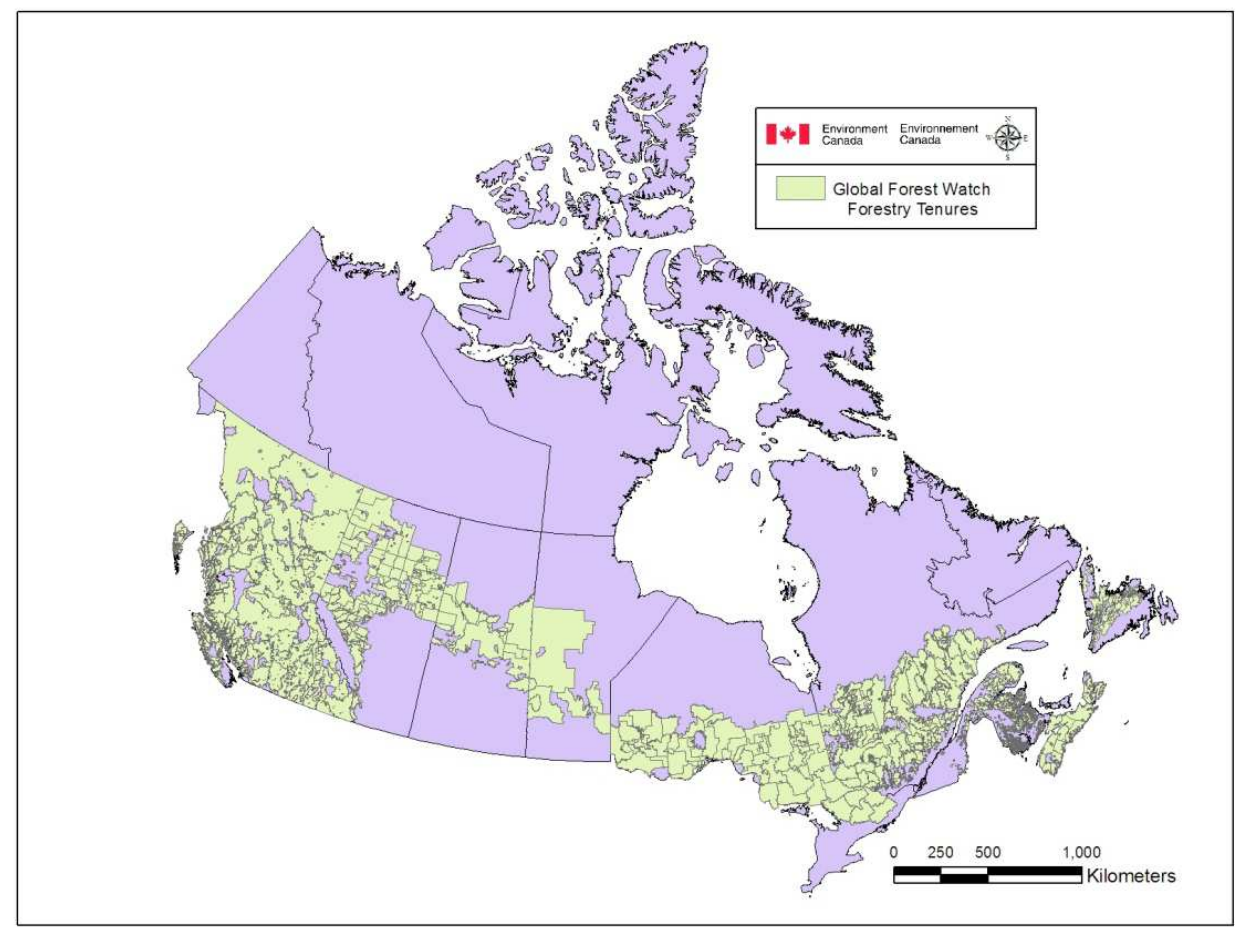

Fig. A4.4: Forestry tenures across Canada as mapped by Global Forest Watch. Data are current to 2004.

The result of our forestry analysis is shown in Fig. A4.5.

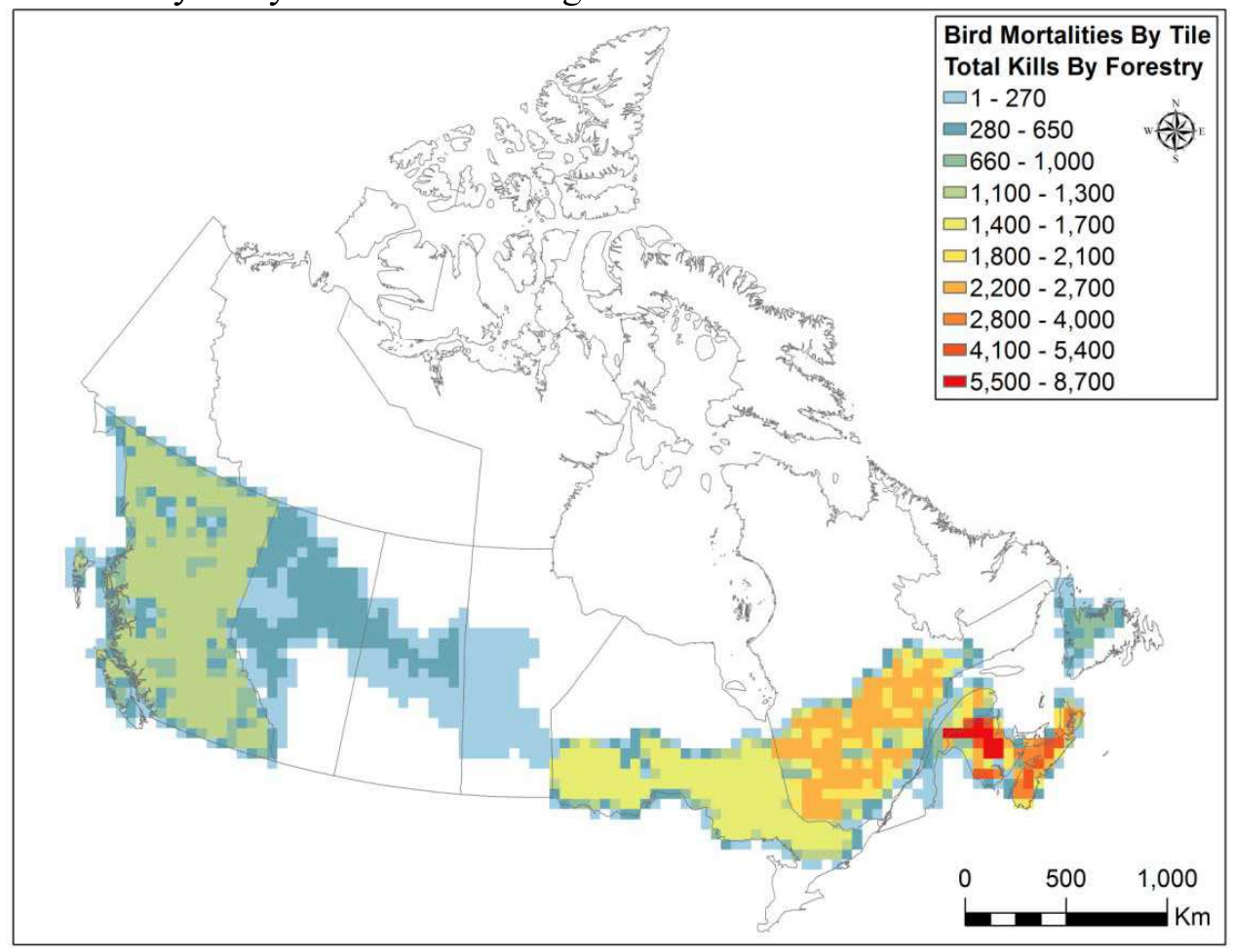

Fig. A4.5 - Approximated distribution of nests destroyed from the forestry sector in Canada as enumerated by Hobson et al. (2013). 


\section{Agriculture}

- Source: Statisics Canada 2006 Agriculture Census (http://www.statcan.gc.ca/ca-ra2006/indexeng.htm) tabular data (no shapefiles)

- Spatial Reference: $\mathrm{n} / \mathrm{a}$

- Ellipsoid Name: $\mathrm{n} / \mathrm{a}$

- Total kill figures (nestlings or eggs) taken from data appendices of Tews et al. (2013):

Table A4.2 - Total kills of nestlings/eggs for five species from data appendix of Tews et. al. (2013) broken down by BCR and type of farming. BCR and grand totals match Table 3 of Tews et al. (2013).

\begin{tabular}{crrrr}
\hline BCR & \multicolumn{1}{c}{ Grain } & \multicolumn{1}{c}{ Haying } & Vegetable & \multicolumn{1}{c}{ Totals } \\
\hline 5 & 1 & 209,913 & 0 & $\mathbf{2 0 9 , 9 1 5}$ \\
6 & 5,276 & 167,061 & 0 & $\mathbf{1 7 2 , 3 3 7}$ \\
8 & 2,345 & 43,778 & 2810 & $\mathbf{4 8 , 9 3 3}$ \\
9 & 1 & 7,460 & 0 & $\mathbf{7 , 4 6 1}$ \\
10 & 31 & 36,602 & 0 & $\mathbf{3 6 , 6 3 3}$ \\
11 & 12,075 & 610,821 & 0 & $\mathbf{6 2 2 , 8 9 7}$ \\
12 & 5,079 & 155,270 & 1,672 & $\mathbf{1 6 2 , 0 2 1}$ \\
13 & 22,777 & 618,698 & 49,252 & $\mathbf{6 9 0 , 7 2 7}$ \\
14 & 41,568 & 203,789 & 13,119 & $\mathbf{2 5 8 , 4 7 7}$ \\
Totals & $\mathbf{8 9 , 1 5 5}$ & $\mathbf{2 , 0 5 3 , 3 9 3}$ & $\mathbf{6 6 , 8 5 3}$ & $\mathbf{2 , 2 0 9 , 4 0 1}$ \\
\hline
\end{tabular}

Agriculture Census information: http://www.statcan.gc.ca/ca-ra2006/about-apropos-eng.htm

\section{Methodology}

Tabular agricultural data from the 2006 Agricultural Census was manually combined with the Canadian Census Subdivision (CD) data (http://www12.statcan.gc.ca/censusrecensement/2011/geo/bound-limit/bound-limit-2011-eng.cfm). The number of each farm types that were present within that CD was entered from the Agriculture Census Data tables. 


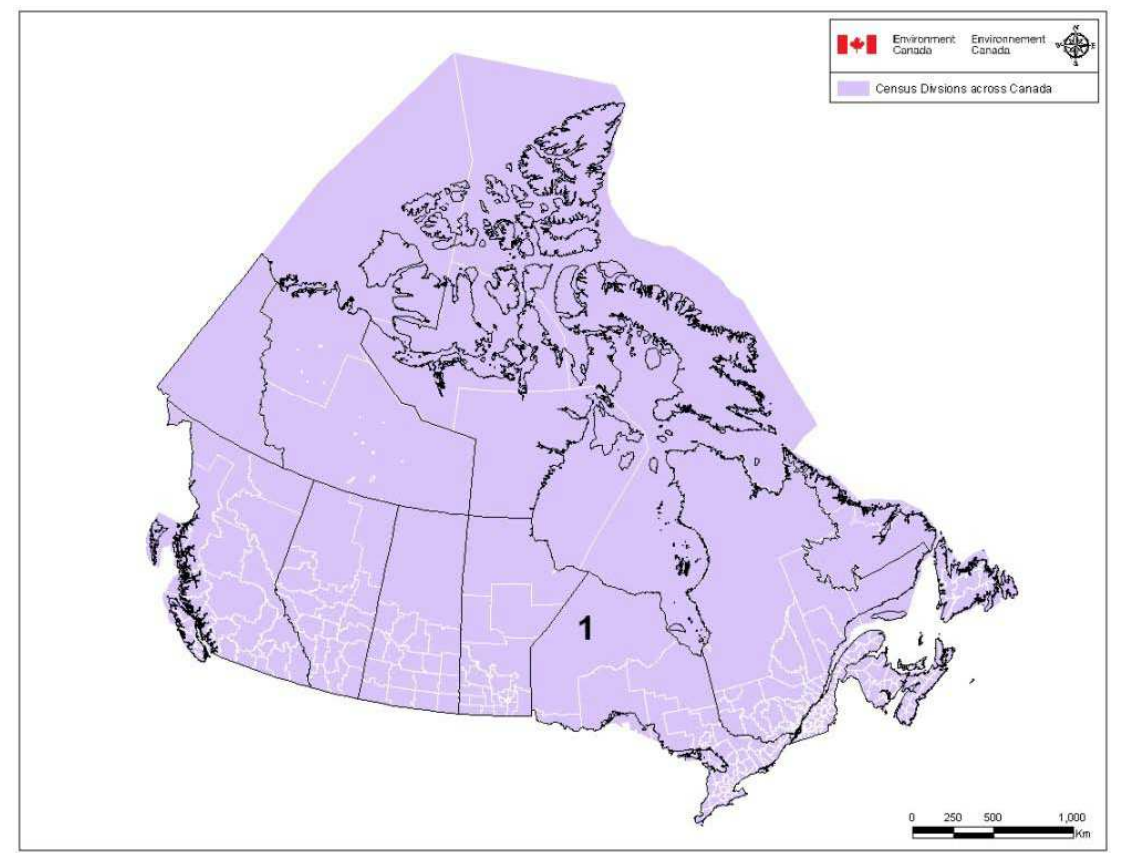

Fig. A4.6 - Census divisions (CDs) of Canada from Statistics Canada. Note that the northern portion of most provinces and all of the territories have very large divisions. The CD labeled with a ' 1 ' is Kenora and is discussed in the text as an example of how the large divisions were problematic.

Below is the list of farm types that have been identified in the Agriculture Census Data. The number in front of each is how we classified them into the aggregate groups following the main list:

2 Hay Farming

3 Fruit and vegetable combination farming

3 All other miscellaneous crop farming

3 Floriculture Production

3 Tobacco Farming

3 Other food crops grown under cover

5 Nursery and tree production

3 Fruit and tree nut farming

3 Mushroom Production

6 Livestock combination farming

4 All other miscellaneous animal production

4 Horse and other equine production

4 Fur bearing animal and rabbit production

6 Goat Farming

5 Apiculture

4 Other poultry production

6 Sheep farming

4 Poultry Hatcheries
3 Potato Farming

3 Other Vegetables (except potatoes) and melon farming

1 Corn farming

1 Other grain farming

1 Dry pea and bean farming

1 Wheat Farming

1 Soybean Farming

1 Oilseed (except soybean) farming

4 Combination poultry and egg production

4 Broiler and other meat type chicken production

4 Turkey Production

4 Hog Pig Farming

4 Chicken Egg Production

4 Dairy Cattle Milk Production

6 Beef Cattle Ranching Farming Feedlots 
Aggregated Groups:

1 - annual grain type cropping

2 - hay farming

3 - fruit, vegetable, other miscellaneous farming

4 - animal farming, not big grazers

5 - farming without regular harvesting (bees, tree growing)

6 - livestock/grazers

Tews et al. (2013) only calculated mortality estimates for the first 3 groups above. Mortality from grazing was calculated by Bleho et al. (2013) separately and is not mapped.

\section{Methodology}

Since there were no geospatial files available with this agricultural data, the CD's geospatial data was downloaded from Statistics Canada in order to link the unique CD ID's with the CD ID's from the 2006 Census of Agriculture dataset. The datasets were merged by using the 'join' operation based on the CD Unique ID's.

CDs in Canada are not equally sized (Fig. A4.6), nor particularly representative of areas of the country where agriculture occurs (Fig. A4.7). This creates a problem as the number and type of farms is only summarized at the CD level. For large CDs with very little agriculture (such as Kenora in Fig. A4.6), we initially had uniformly distributed the farm count across all of our $50 \times 50 \mathrm{~km}$ tiles covering the entire CD. The result of that process was that it appeared as though hay farming occurred everywhere at a low level in Ontario, from the US border right to James Bay.

To fix this problem we constrained our $50 \times 50 \mathrm{~km}$ tile grid by the extent of agriculture land in Canada. The tile layer was intersected with the agriculture lands layer (Fig. A4.7), producing a much smaller potential tile grid in Canada. This is identical to the process used to display forestry in Canada where our tile grid was constrained by the forestry tenure layer. The result is that large and/or northern CDs like Kenora now only had grid tiles where agriculture occurs (in this case at the extreme southern end). 


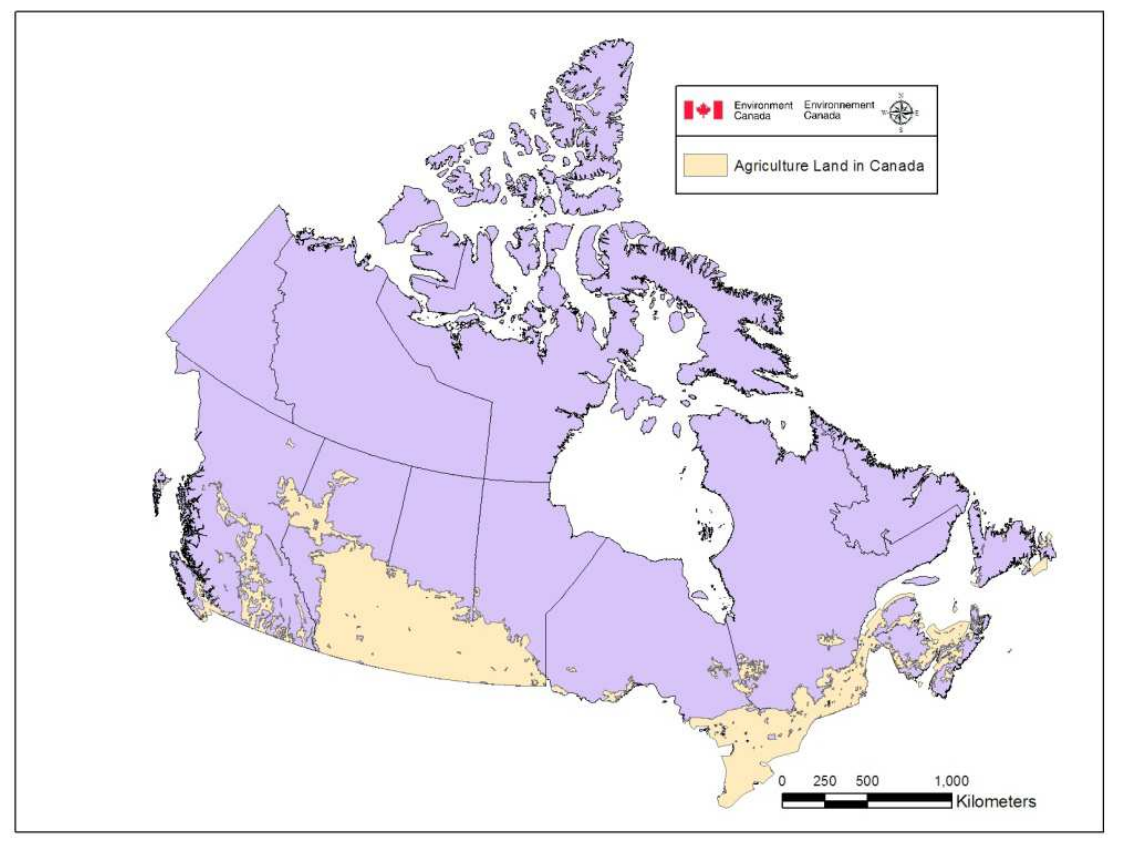

Fig. A4.7 - Distribution of agricultural land in Canada. Data provided by T. Rounce, Agriculture and Agri-Food Canada. Original derivation is from the Census of Agriculture interpolated variable called 'PERAGR' which is the percent of agriculture within a Soil Landscape of Canada (v3.1) polygon.

Agricultural data was displayed by $\mathrm{CD}$ across Canada. In order to determine the total percentage of each farm type that occurs by tile across Canada we did the following:

- Census Divisions were intersected to each $50 \times 50 \mathrm{~km}$ tile. The sub-area for each divided CD (CDs crossing tile boundaries) was calculated and the area value was then used to calculate the percentage of each CD's that fell within each tile (assumes population is homogenously distributed in each $C D$, likely not true).

- The number of each farm type within each CD (Grain, Haying, Vegetables) was multiplied by the proportion of each CD that fell within each unique tile ID in order to obtain the number of farms within each tile for each farm type.

- The 'Dissolve' tool was then used so that all CD's were dissolved by each $50 \times 50 \mathrm{~km}$ tile and the statistics field was used to 'SUM' the number of farms within each tile. This was done separately for each of the 3 farm types.

- Ideally we need the area of each farm type in each CD. However, Statistics Canada does not provide the area associated with each farm or in aggregate, so all we have is a count of farms by type in each CD.

- Equally distribute the quotient to each tile.

A map of Bird Conservation Regions (BCRs) was then overlaid on the tile grid with all the farming data. The two layers were intersected and each tile was assigned to its respective BCR (overlap cases were put into the BCR which contained the greater proportion of the tile area). The total number of farms of each type for all tiles in each BCR was calculated and the proportion in each tile was then derived.

Kills by farm type and BCR (Table A4.2) were then multiplied by the proportion of that farm type in that BCR for each $50 \times 50 \mathrm{~km}$ tile. This represents the total nestlings or eggs killed per tile. 
The results of our agriculture analysis are shown in Fig. A4.8: Grain Cropping, Fig. A4.9: Haying, and Fig. A4.10: Vegetable Cropping.

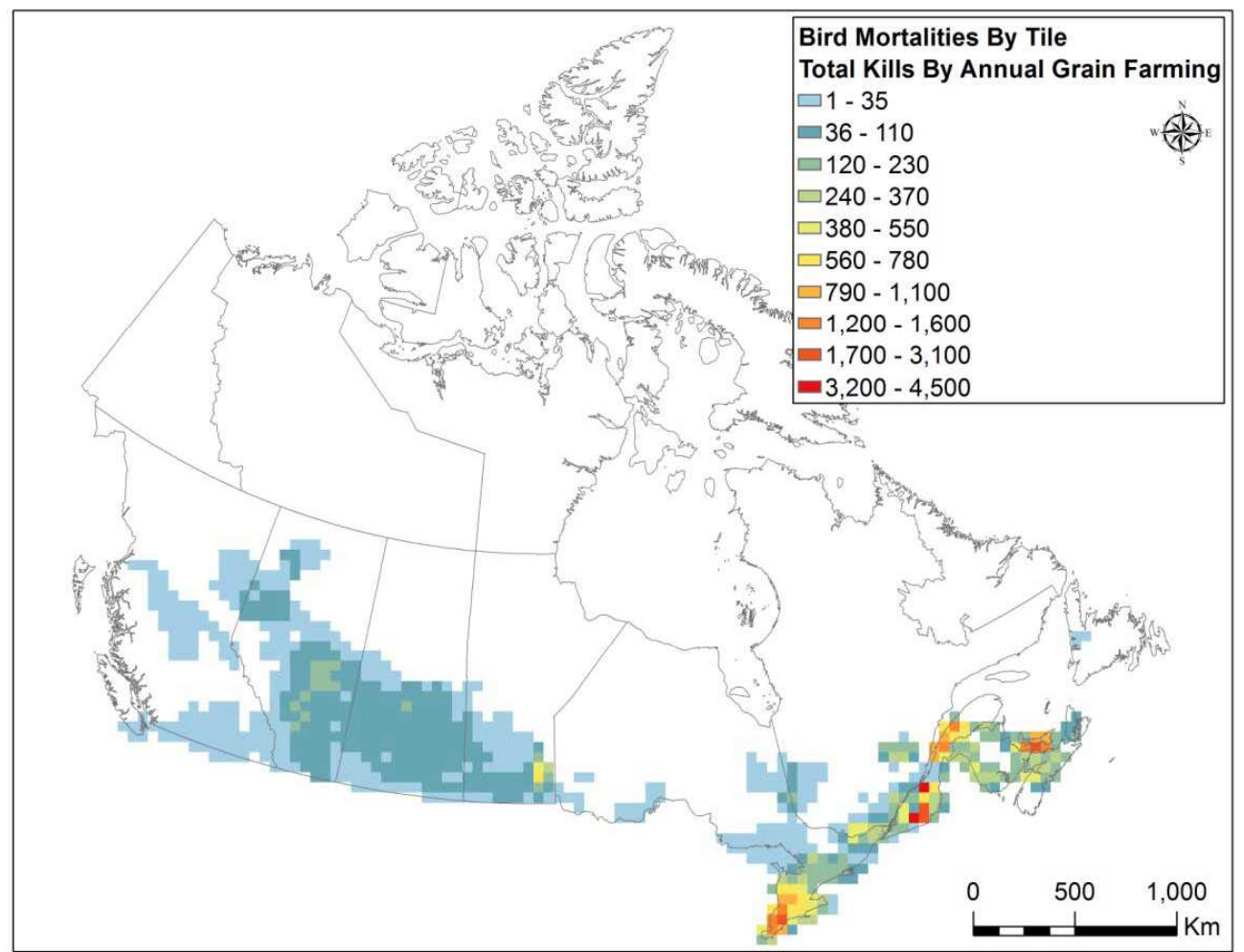

Fig. A4.8 - Approximated distribution of nestlings and eggs destroyed from the grain cropping in Canada as enumerated by Tews et al. (2013). 


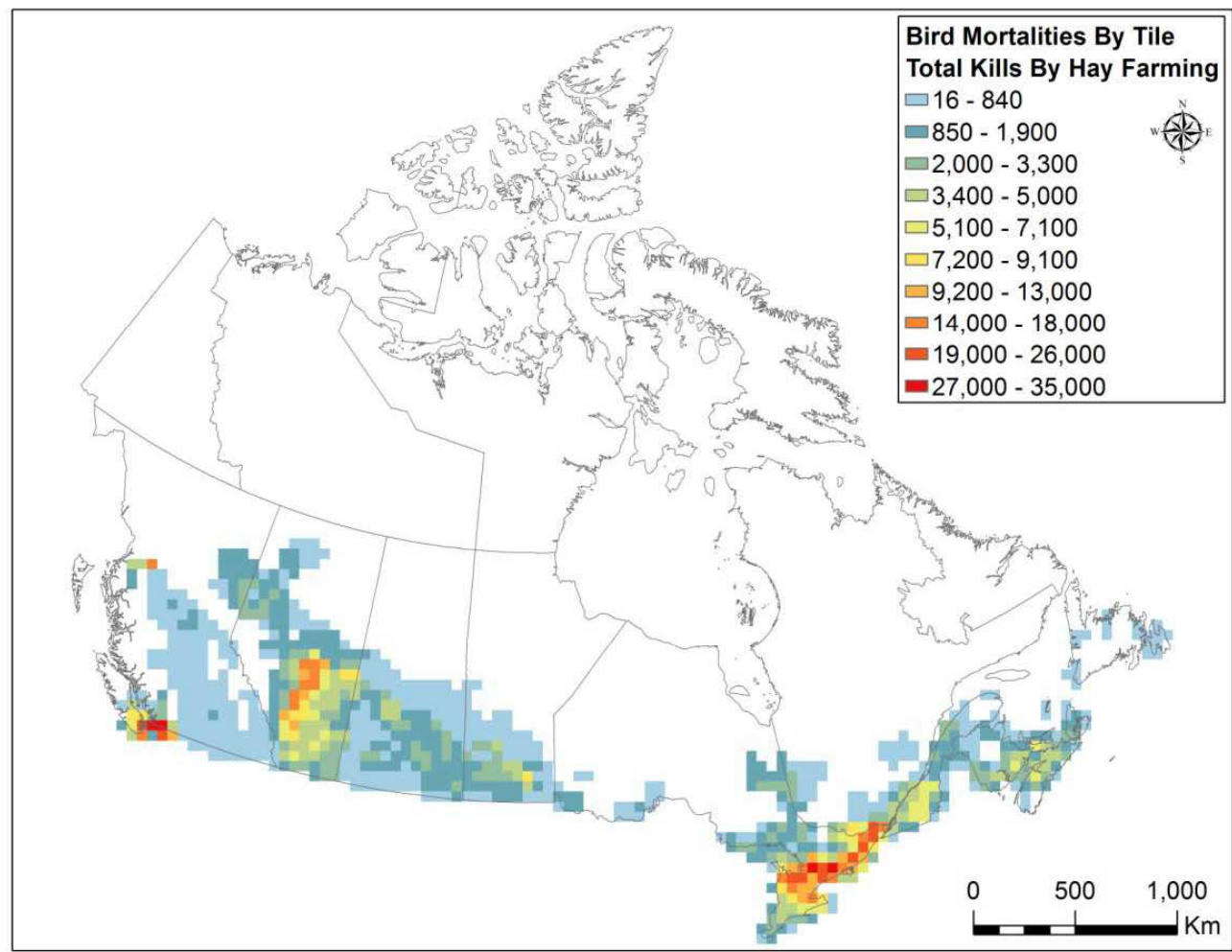

Fig. A4.9 - Approximated distribution of nestlings and eggs destroyed from the haying in Canada as enumerated by Tews et al. (2013).

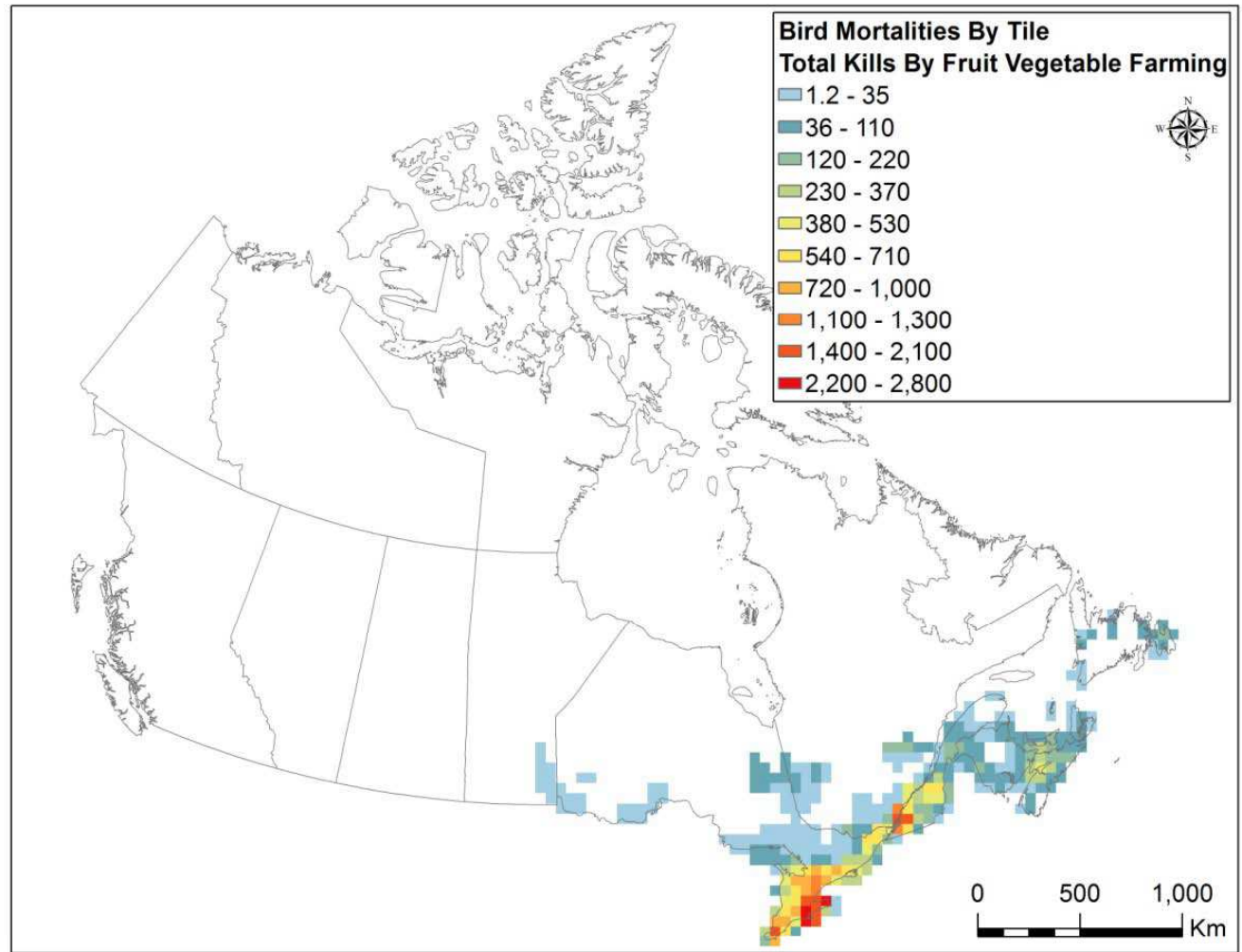

Fig. A4.10 - Approximated distribution of nestlings and eggs destroyed from the vegetable (and fruit) cropping in Canada as enumerated by Tews et al. (2013). Agriculture Census data had entries fruit and vegetable farms in western Canada (as expected), but the species analyzed and results from Tews et al. 
(2013) had zero deaths for the western BCRs (such as BCRs 5 and 9 covering the lower mainland of $\mathrm{BC})$.

\section{Cats and Buildings (based on Population Density)}

- Source: 2006 Census of Human Population, Statistics Canada (http://www12.statcan.gc.ca/census-recensement/2006/index-eng.cfm)

- Spatial Reference: $\mathrm{n} / \mathrm{a}$

- Ellipsoid Name: $\mathrm{n} / \mathrm{a}$

- Total kill figure used: Cats: 204 million (median value, Blancher 2013); Buildings: 24,900,000 (total average values from Table 5 in Machtans et al. (2013).

The 2006 Census Population Density data for Canada was used to display the data by Census Division (CD). The Census Division data was not spatially represented so the geospatial file containing the Census Divisions for Canada were also obtained from Statistics Canada in order to acquire the desired results.

\section{Methodology}

Human population density was used as a proxy for the spatial distribution of both cats and buildings across Canada. We provide rationale below for using population data as the proxy for the two sources of mortality.

Cats: Per capita pet ownership in Canada was tabulated by Leger Marketing (http://www.legermarketing.com/documents/SPCLM/020617eng.pdf) and varied somewhat across Canada. In the absence of spatial data on pet ownership however, we calculated $\leq 2 \%$ difference between the proportion of cats per province (number of households $\times \%$ cat ownership) and the $\%$ of total human population per province (Table A4.3). Although the results are autocorrelated from using population to calculate cats, this exercise demonstrates that the regional variation in cat ownership rates is a trivial deviation from simply approximating ownership by population. We recognize that if the number of cats per household varies radically across the country that it would introduce another inaccuracy into our map (Perrin [2009] indicates an average of 1.76 cats per owner). We expected unowned cat numbers to be associated with owned cats in some unknown, but constant ratio (fewer people in rural settings, fewer pet cats total, and fewer feral cats, with the opposite in cities). We did not explicitly extrapolate the unowned cats into the totals below. Because we assumed a constant ratio between the two types, and allocated the total kill of all cats to the proportional representation of population, no mapping differences would appear from calculating unowned cats in the table below.

Table A4.3: Comparison of geographic distribution of population by province and calculated owned cats per province.

\begin{tabular}{cccccccc}
\hline Prov & $\begin{array}{c}\text { Population } \\
(2011)\end{array}$ & $\begin{array}{c}\text { Proportional } \\
\text { Population }\end{array}$ & $\begin{array}{c}\text { People/ } \\
\text { Household }\end{array}$ & $\begin{array}{c}2002 \text { Cats } \\
\text { Ownership } \\
\text { Rate }\end{array}$ & $\begin{array}{c}\text { Number } \\
\text { of Owned } \\
\text { Cats }\end{array}$ & $\begin{array}{c}\text { Proportional } \\
\text { Cat } \\
\text { Population }\end{array}$ & $\begin{array}{c}\text { Difference } \\
(\% \\
\text { Human - } \\
\% \text { Cat })\end{array}$ \\
\hline BC & $4,400,057$ & $13 \%$ & 2.5 & $32 \%$ & 991,245 & $15 \%$ & $-2 \%$ \\
AB & $3,645,257$ & $11 \%$ & 2.6 & $31 \%$ & 764,943 & $11 \%$ & $0 \%$ \\
SK & $1,033,381$ & $3 \%$ & 2.4 & $21 \%$ & 159,141 & $2 \%$ & $1 \%$
\end{tabular}




\begin{tabular}{cccccccc}
\hline \hline Prov & $\begin{array}{c}\text { Population } \\
(2011)\end{array}$ & $\begin{array}{c}\text { Proportional } \\
\text { Population }\end{array}$ & $\begin{array}{c}\text { People/ } \\
\text { Household }\end{array}$ & $\begin{array}{c}\text { 2002 Cats } \\
\text { Ownership } \\
\text { Rate }\end{array}$ & $\begin{array}{c}\text { Number } \\
\text { of Owned } \\
\text { Cats }\end{array}$ & $\begin{array}{c}\text { Proportional } \\
\text { Cat } \\
\text { Population }\end{array}$ & $\begin{array}{c}\text { Difference } \\
\text { Human - } \\
\% \text { Cat })\end{array}$ \\
\hline MB & $1,208,268$ & $4 \%$ & 2.5 & $21 \%$ & 178,630 & $3 \%$ & $1 \%$ \\
ON & $12,851,821$ & $38 \%$ & 2.6 & $28 \%$ & $2,435,914$ & $36 \%$ & $2 \%$ \\
QC & $8,080,550$ & $24 \%$ & 2.3 & $26 \%$ & $1,607,678$ & $24 \%$ & $0 \%$ \\
NB & 751,171 & $2 \%$ & 2.4 & $37 \%$ & 203,818 & $3 \%$ & $-1 \%$ \\
NF & 514,536 & $2 \%$ & 2.5 & $37 \%$ & 134,026 & $2 \%$ & $0 \%$ \\
NS & 921,727 & $3 \%$ & 2.4 & $37 \%$ & 250,095 & $4 \%$ & $-1 \%$ \\
PEI & 140,204 & $0 \%$ & 2.5 & $37 \%$ & 36,520 & $1 \%$ & $0 \%$ \\
NT & 41,462 & $0 \%$ & & & & & \\
NU & 31,906 & $0 \%$ & & & & & \\
YT & 33,897 & $0 \%$ & & & & & \\
\hline
\end{tabular}

Notes:

1. People/household is from 2006 Population Census, statistics Canada.

2. Average cat ownership rate was higher (about $36 \%$ compared to $31 \%$ here) in Perrin (2009) as cited by Blancher (2013), but we could not get updated geographic breakdowns of ownership rate and therefore used the 2002 figures from the Leger survey. Therefore the sum of the column "number of cats" is lower than the estimated number of pet cats given in Blancher (2013).

Buildings: The number of people per household in Canada varies little $(2.3-2.6$ persons per household for all major provinces [2006 Census data]), and since each household should be a unit represented in the analysis of Machtans et al. (2013), the proxy of population for buildings is gross (not 1:1), but suitable for broad scale mapping. This relationship would be confounded slightly since high rise apartments have many households in them and are technically counted in the high rise buildings section of Machtans et al. (2013). However, we did not break out the three categories of buildings separately in the mapping exercise so this confounding would be largely inconsequential. We did not have data to correlate the number of low-rise commercial buildings to population but assumed it was a linear relationship so proportional population could represent the kills from this class.

The number of tall buildings is highly correlated with $\log$ (population) in cities but is not completely log-linear (Fig. A4.11). We ignored both the logarithmic curve function and its inflection given that of the $\sim 25 \mathrm{M}$ birds killed by buildings in Canada, only $1 \%$ in total is ascribed to tall buildings and slight adjustments for the proportion of buildings in each city in Canada in each $50 \times 50 \mathrm{~km}$ tile would not be visible in the final map. While technically this would have been a better map of tall building density, Machtans et al. (2013) provides evidence that the number of buildings alone is a poor predictor of tall building bird-collision deaths (geography seems very important). 


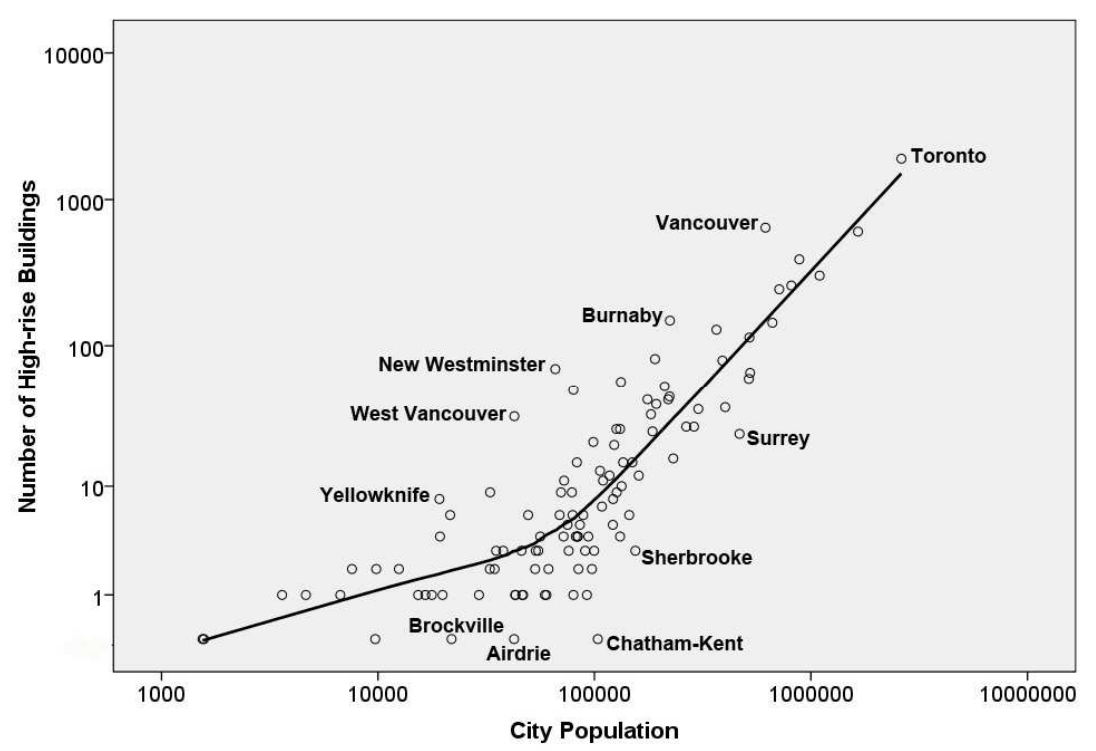

Fig. A4.11 - The relationship between city population and number of high-rise buildings in Canada. High-rise data from http://skyscraperpage.com/cities/?s=0\&c $=2 \& p=0 \& r=50 \& 10=0$

Population data were exported from Statistics Canada and merged together for all CD's in Canada. Tabular data were joined with the CD shapefile which contained the polygons for each $C D$. The join was performed based on the unique ID's from the CD's ID field. Using the 'Field Calculator', the area for each $C D$ was calculated.

Census Divisions were intersected to each $50 \times 50 \mathrm{~km}$ tile. The sub-area for each divided CD (CDs crossing tile boundaries) was calculated and the area value was then used to calculate the percentage of each CD's that fell within each tile (assumes population is homogenously distributed in each CD, likely not true). The result was multiplied by the 2006 Population Density for each unique CD based on the percentage of the CD's that fall within each individual tile.

Census Divisions were dissolved by each $50 \times 50 \mathrm{~km}$ tile and population densities were summed. Proportion of the total national population in each $50 \times 50 \mathrm{~km}$ tile was calculated.

Proportional population in each tile was multiplied by the kill values for cats and buildings separately to produce the two data layers. The problem with large census divisions explained in the Agriculture section was also encountered for the population layer. We arbitrarily removed all tiles with population densities of $<100$ people per tile $\left(0.04 / \mathrm{km}^{2}\right)$. We then reinserted only the tiles that contained communities.

The results of our analyses (Fig. A4.12: cats; Fig. A4.13: buildings) appear identical because the 10 colours chosen for each class are identical and the underlying population distribution is the same. Note the different estimate of take per tile in the legends however. 


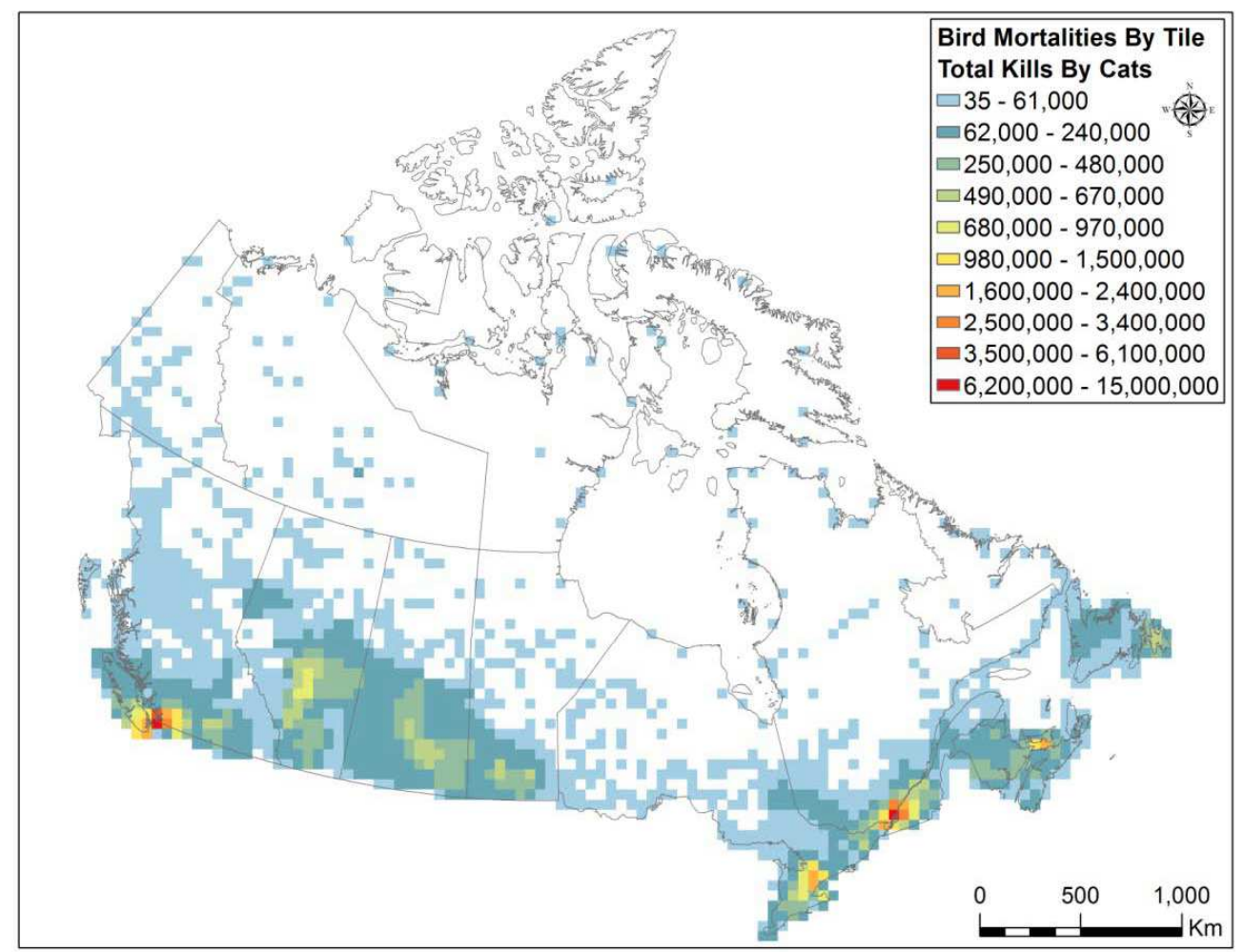

Fig. A4.12 -Approximated distribution of birds killed by cats in Canada from values in Blancher (2013).

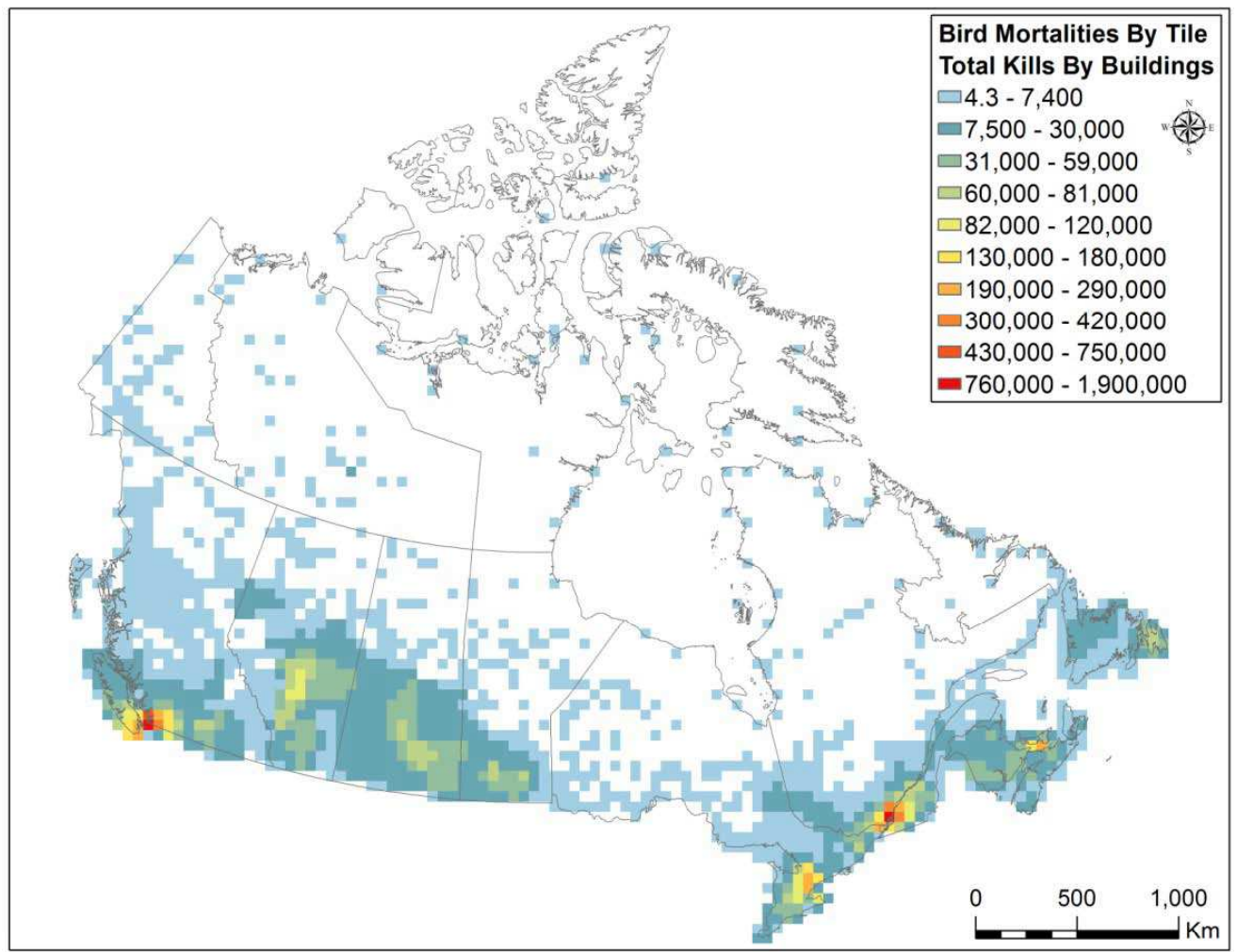

Fig. A4.13 - Approximated distribution of birds killed by colliding with windows in buildings in Canada from values in Machtans et al. (2013). Note that the legend values are different than in the similarly coloured Fig. A4.12. 


\section{Wind Power}

- Source: Canadian Wind Energy Association (http://www.canwea.ca/farms/wind-farms e.php) web table and on-line map

- Spatial Reference: $\mathrm{n} / \mathrm{a}$

- Ellipsoid Name: $\mathrm{n} / \mathrm{a}$

- Total kill figure used: 5.9 birds per turbine from Zimmerling et al. (2013).

We created our own point layer shapefile by deriving coordinates for each wind farm from the above noted source. We entered the provided data for each wind farm (number of turbines and type) in the attribute file.

\section{Methodology}

Each point in our wind farm layer (one wind farm) was assigned a total kills by multiplying the number of turbines at that facility by the average kills per turbine noted above. The $50 \times 50 \mathrm{~km}$ tile grid was overlaid with the wind farm point layer and a spatial join was performed in order to assign a tile ID to each wind farm point location. The Dissolve tool was then run to sum all kills within each unique tile ID using the statistics filed within the dissolve tool.

The result of our analysis on wind farms is in Fig. A4.14.

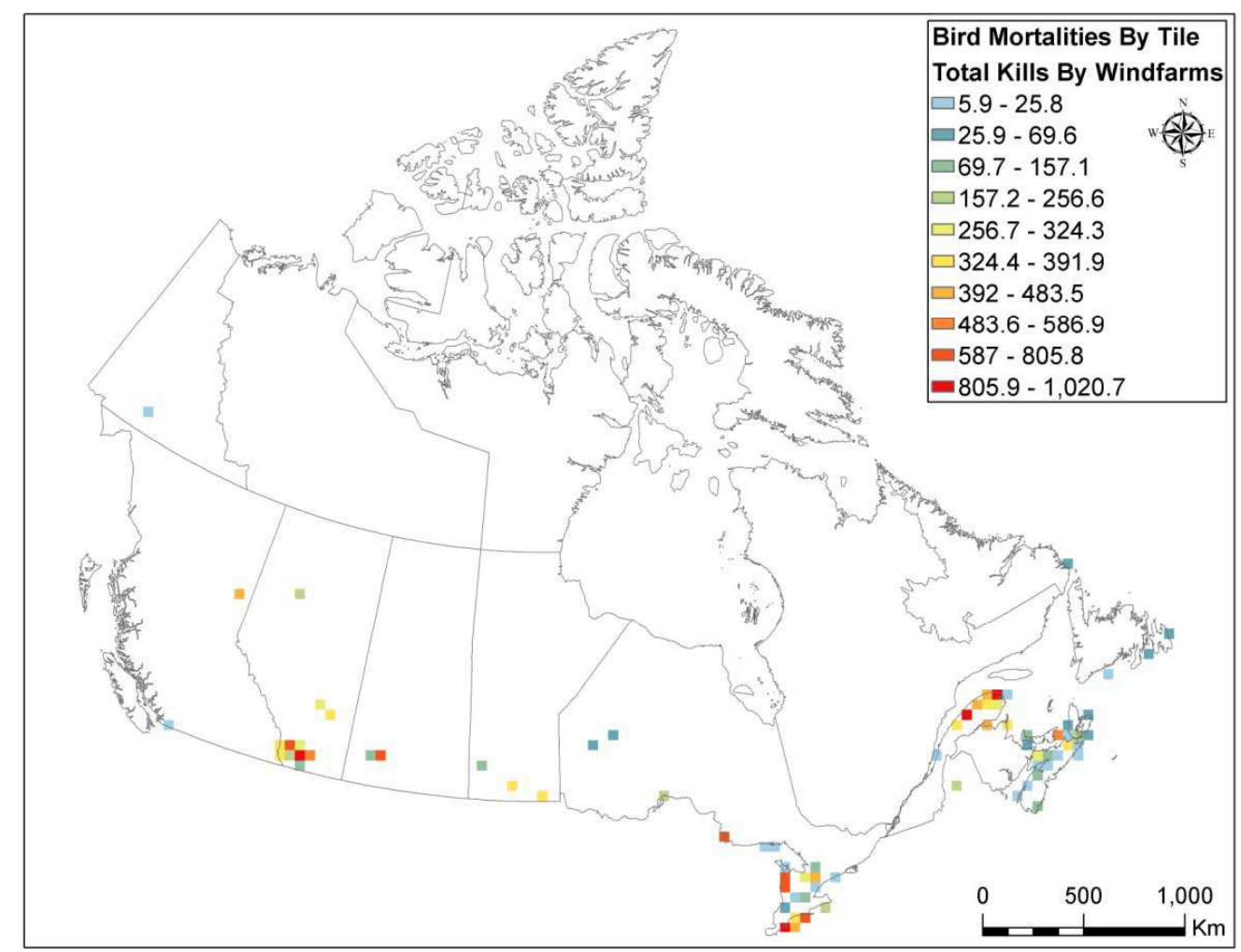

Fig. A4.14: Approximated distribution of kill of birds by wind farm operation in Canada based on average kill per turbine in Zimmerling et al. (2013). 


\section{Combined layers used in main paper}

The final analysis involves summing all tile values for each of the specified mortality sources. Two maps were created. First, a map summing all layers was made. However, since so much kill in Canada is attributed to cats, bird-building collisions, and bird-vehicle collisions, a second map without these three sources was created.

The results of the summed layers for all mortality sources are shown in Fig. A4.15 and the results without the three sources noted above are in Fig. A4.16. Finally, the distribution of total mortality from only the top three sources (cats, bird-building collisions and bird-vehicle collisions) is presented in Fig. A4.17.

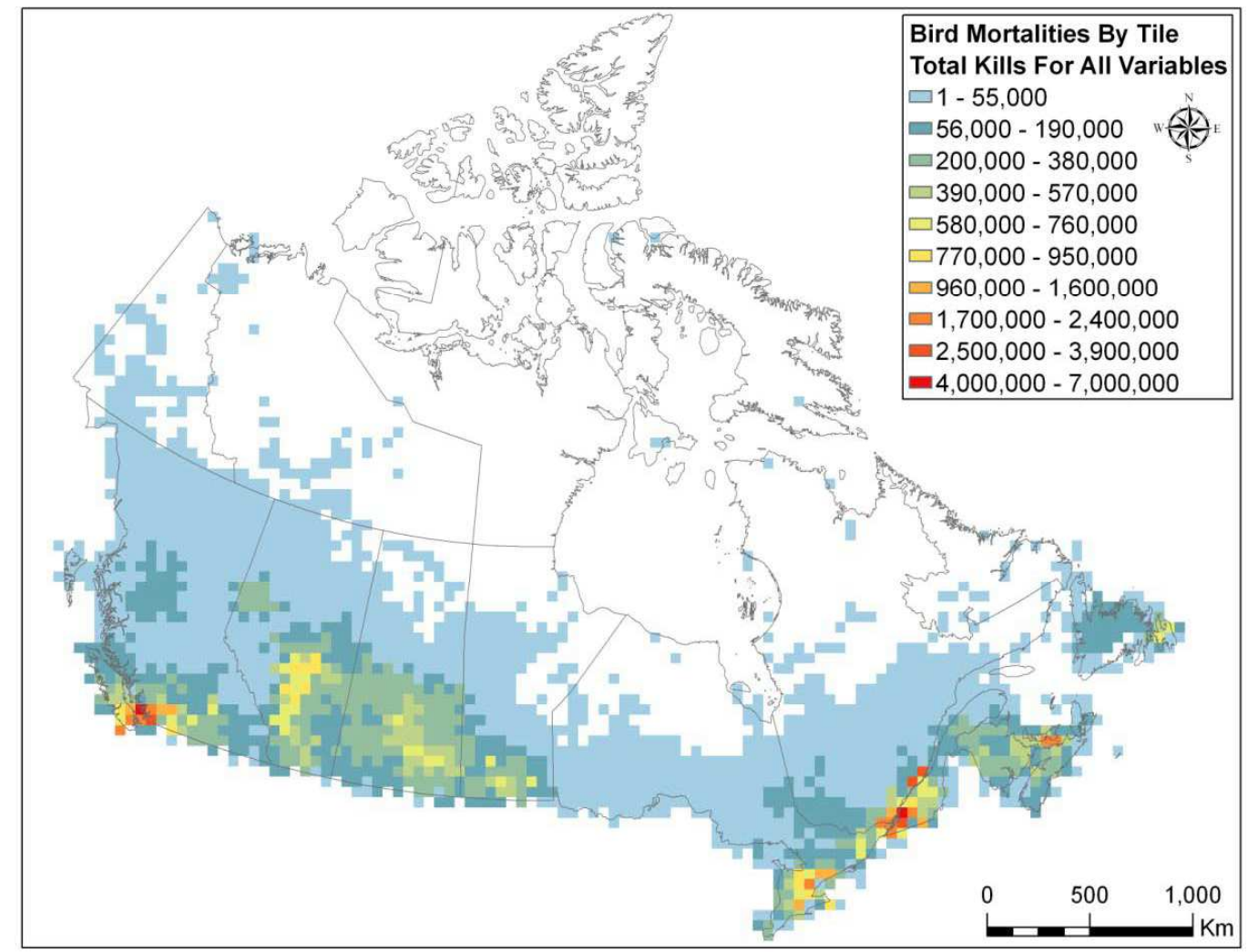

Fig. A4.15 - Approximated distribution of all bird mortalities across all sources considered. 


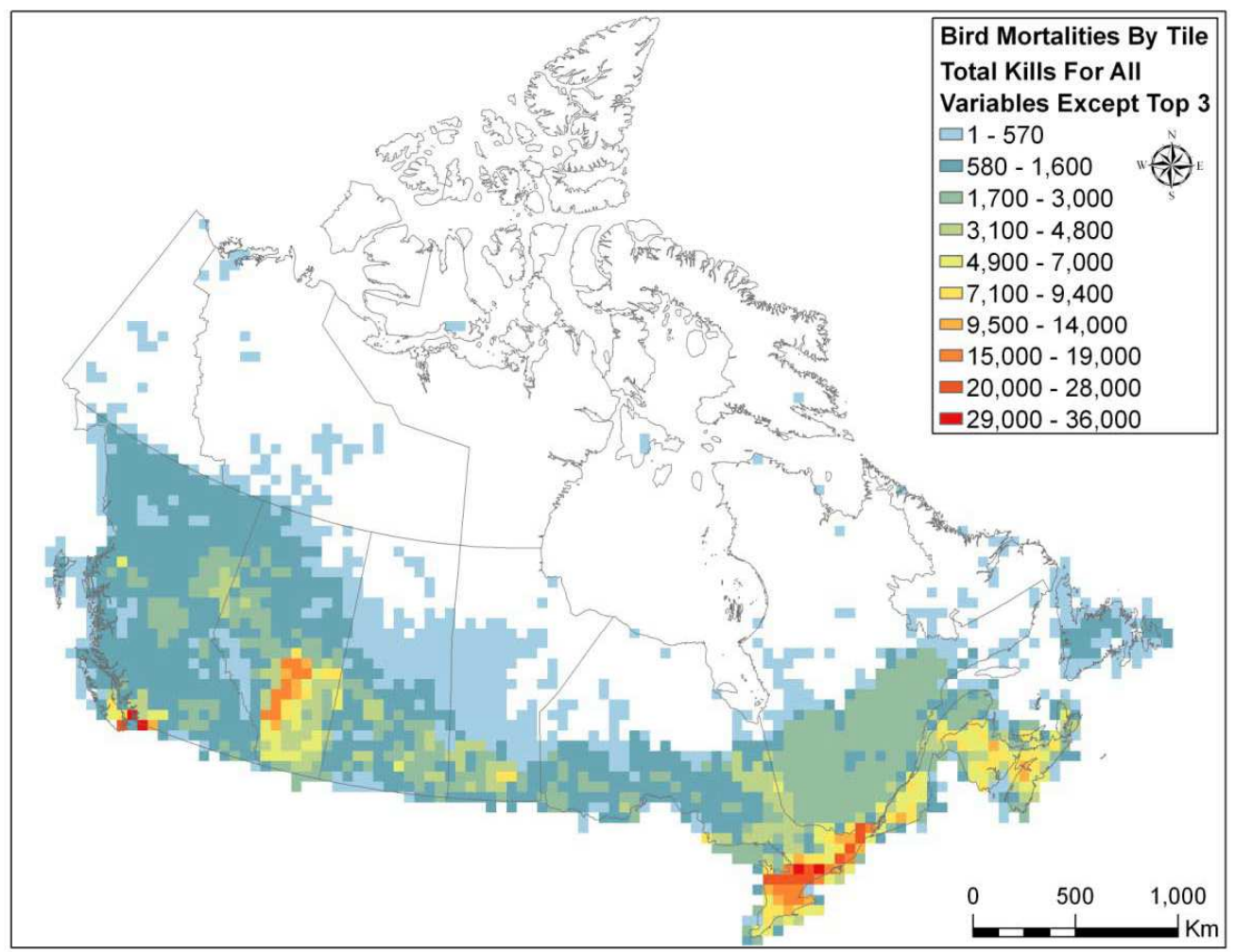

Fig. A4.16: Approximated distribution of all bird mortalities for all sources except cats, bird-building collisions and bird-vehicle collisions.

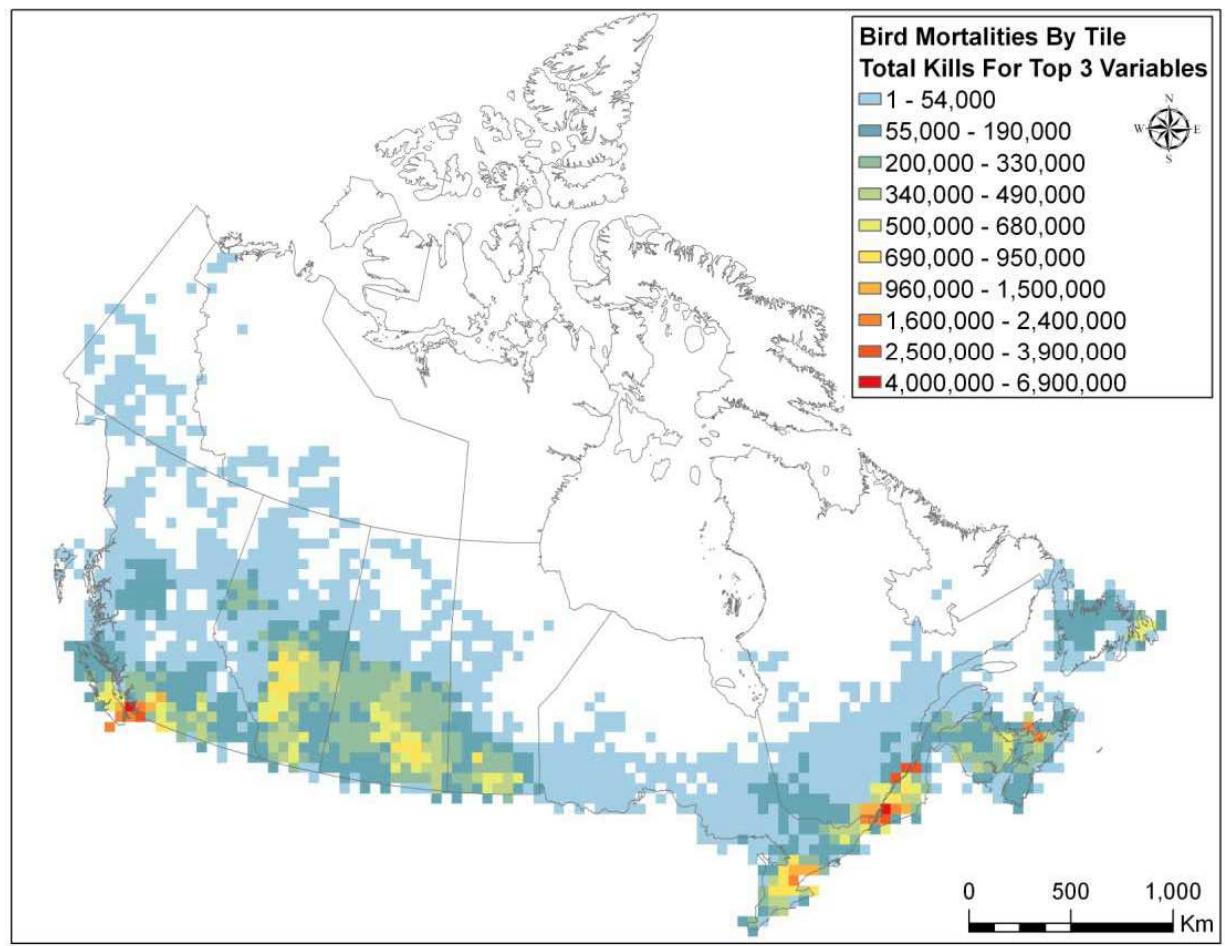

Fig. A4.17 - Approximated distribution of all bird mortalities from cats, bird-building collisions and bird-vehicle collisions only. 


\section{Literature Cited (Appendix 4)}

Bishop, C. A., and J. M. Brogan. 2013. Estimates of avian mortality due to vehicle collisions on the Canadian road network. Avian Conservation and Ecology (this issue).

Blancher, P. J. 2013. Estimated number of birds killed by house cats (Felis catus) in Canada. Avian Conservation and Ecology (this issue).

Bleho, B., N. Koper, and C. Machtans. Submitted. Effects of incidental take of nests by livestock on grassland birds. Submitted to Conservation Biology, August 2012.

Hobson, K. A., A. Wilson, S. L. Van Wilgenburg, and E. Bayne. 2013. An estimate of nest loss in Canada due to industrial forestry operations. Avian Conservation and Ecology (this issue).

Longcore, T., C. Rich, P. Mineau, B. MacDonald, D. G. Bert, L. M. Sullivan, E. Mutrie, S. A. Gauthreaux Jr., M. L. Avery, R. L. Crawford, A. M. Manville II, E. R. Travis, and D. Drake. 2012. An estimate of avian mortality at communication towers in the United States and Canada. PLoS ONE 7(4): e34025. doi:10.1371/journal.pone.oo34025

Machtans, C. S., C. H. R. Wedeles, and E. M. Bayne. 2013. A first estimate for Canada of the number of birds killed by colliding with building window. Avian Conservation and Ecology (this issue).

Perrin, J. 2009. The Business of Urban Animals Survey: The facts and statistics on companion animals in Canada. Canadian Veterinary Journal 50:48-52.

Taylor, W. A. 1975. A History of Survey Systems. Ministry of Sustainable Resource Management. Victoria, British Columbia.

Tews, J., D. G. Bert, and P. Mineau. 2013. Estimated mortality of selected migratory bird species from mowing and other mechanical operations in Canadian agriculture. Avian Conservation and Ecology (this issue).

Van Wilgenburg, S. L., K. A. Hobson, E. M. Bayne, and N. Koper. 2013. Estimated avian nest loss associated with oil and gas exploration and extraction in the Western Canadian Sedimentary Basin. Avian Conservation and Ecology (this issue).

Zimmerling, J. R., A. Pomeroy, M. d'Entremont, and C. M. Francis. 2013. Canadian estimate of bird mortality due to collisions and direct habitat loss associated with wind turbine developments. Avian Conservation and Ecology (this issue). 